\title{
Lifestyle factors and risk of head-neck cancer subtypes
}

Citation for published version (APA):

Maasland, D. H. E. (2016). Lifestyle factors and risk of head-neck cancer subtypes: a prospective cohort study. [Doctoral Thesis, Maastricht University]. Maastricht University. https://doi.org/10.26481/dis.20161005dm

Document status and date:

Published: 01/01/2016

DOI:

$10.26481 / \mathrm{dis} .20161005 \mathrm{dm}$

Document Version:

Publisher's PDF, also known as Version of record

\section{Please check the document version of this publication:}

- A submitted manuscript is the version of the article upon submission and before peer-review. There can be important differences between the submitted version and the official published version of record.

People interested in the research are advised to contact the author for the final version of the publication, or visit the DOI to the publisher's website.

- The final author version and the galley proof are versions of the publication after peer review.

- The final published version features the final layout of the paper including the volume, issue and page numbers.

Link to publication

\footnotetext{
General rights rights.

- You may freely distribute the URL identifying the publication in the public portal. please follow below link for the End User Agreement:

www.umlib.nl/taverne-license

Take down policy

If you believe that this document breaches copyright please contact us at:

repository@maastrichtuniversity.nl

providing details and we will investigate your claim.
}

Copyright and moral rights for the publications made accessible in the public portal are retained by the authors and/or other copyright owners and it is a condition of accessing publications that users recognise and abide by the legal requirements associated with these

- Users may download and print one copy of any publication from the public portal for the purpose of private study or research.

- You may not further distribute the material or use it for any profit-making activity or commercial gain

If the publication is distributed under the terms of Article $25 \mathrm{fa}$ of the Dutch Copyright Act, indicated by the "Taverne" license above, 


\section{LIFESTYLE FACTORS AND RISK OF HEAD-NECK CANCER SUBTYPES: \\ a prospective cohort study}

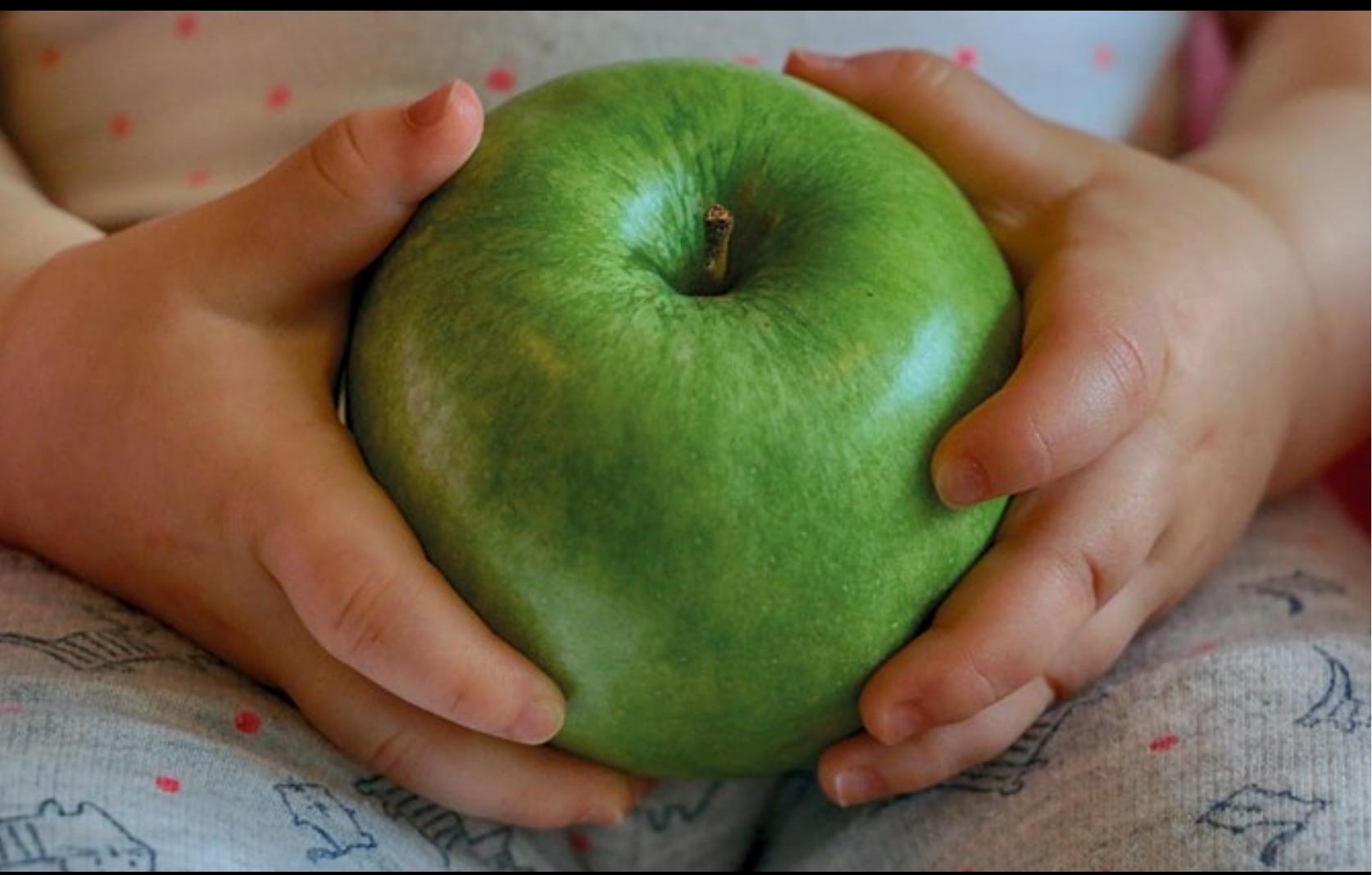

Denise Maasland 

Lifestyle factors and risk of head-neck cancer subtypes: a prospective cohort study 
The research described in this thesis was conducted within the GROW - School for Oncology and Developmental Biology at the Department of Epidemiology, Maastricht University, in collaboration with the Department of Otorhinolaryngology, Head \& Neck Surgery, Maastricht University Medical Center+, Maastricht, The Netherlands.

Copyright @ 2016 D.H.E. Maasland, Maastricht, The Netherlands

Lifestyle factors and risk of head-neck cancer subtypes: a prospective cohort study ISBN 978-94-028-0282-5

Cover design and photo: Denise Maasland, Servaas \& Iza van Zij|

Layout: Denise Maasland

Printed by: Ipskamp Printing, Enschede, The Netherlands

All rights reserved. No part of this thesis may be reproduced or transmitted in any form or by any means, electronic or mechanical, including photocopying, recording, or any information storage or retrieval system, without prior permission in writing from the author, or, when appropriate, from the publishers of the publications. 


\title{
Lifestyle factors and risk of head-neck cancer subtypes: a prospective cohort study
}

\author{
PROEFSCHRIFT
}

ter verkrijging van de graad van doctor aan de Universiteit Maastricht, op gezag van de Rector Magnificus, prof. dr. Rianne M. Letschert, volgens het besluit van het College van Decanen, in het openbaar te verdedigen

op woensdag 5 oktober 2016 om 14:00 uur

door

Denise Helena Elisabeth Maasland 


\section{Promotores}

Prof. dr. ir. P.A. van den Brandt

Prof. dr. B. Kremer

\section{Copromotor}

Dr. L.J. Schouten

\section{Beoordelingscommissie}

Prof. dr. E.J.M. Speel (voorzitter)

Dr. B.A.C. van Dijk (Integraal Kankercentrum Nederland)

Prof. dr. P.A.W.H. Kessler

Prof. dr. S.P.J. Kremers

Prof. dr. B.F.A.M. van der Laan (Universitair Medisch Centrum Groningen)

\section{Wereld Kanker

Financial support for this study was kindly provided by the Wereld Kanker Onderzoek Fonds (WCRF-NL). 


\section{Contents}

Chapter 1 Introduction

Chapter 2 Alcohol consumption, cigarette smoking and the risk of subtypes of head-neck cancer: results from the Netherlands Cohort Study BMC Cancer 2014;14:187

Chapter 3 Consumption of vegetables and fruits and risk of subtypes of head-neck cancer in the Netherlands Cohort Study Int J Cancer 2015;136:E396-409

Chapter 4 Vitamin and carotenoid intake and the risk of head-neck cancer 69 subtypes in the Netherlands Cohort Study Am J Clin Nutr 2015;102:420-32

Chapter 5 Toenail selenium status and risk of subtypes of head-neck 95 cancer: the Netherlands Cohort Study

Eur J Cancer 2016;60:83-92

Chapter 6 Body mass index and risk of subtypes of head-neck cancer: the Netherlands Cohort Study

Sci Rep 2015;5:17744

Chapter 7 Discussion

Summary

Samenvatting

Valorization

Dankwoord

Curriculum Vitae 

Chapter 1

Introduction 
This thesis concerns the associations between lifestyle factors and the risk of headneck cancer (HNC) and HNC subtypes. In this chapter, we will first discuss the epidemiology and course of HNC. Subsequently, classification of HNC and HNC risk factors will be addressed. Finally, our study design and rationale, aim, and outline of the thesis will be considered.

\section{Head-neck cancer}

In this paragraph, we will discuss the epidemiology and course of HNC.

\section{Incidence and mortality}

HNC is one of the most frequently occurring cancers. It is the seventh most common type of cancer in the world and Europe, and includes, among others, malignancies originating from the oral cavity, pharynx, and larynx. ${ }^{1,2}$ Worldwide, HNC represents about $6 \%$ of all cancer cases and accounts for an estimated 650,000 new cases and 350,000 cancer deaths every year. ${ }^{1,3}$ More than $90 \%$ of all head and neck cancers are squamous cell carcinomas (SCC). ${ }^{1}$ HNC is more frequent among men than women, especially for laryngeal cancer, and diagnosis of cancer of the mouth, pharynx, and larynx is most common in people aged 50 or over; the median age is around 60 years. ${ }^{1}$ There is a slight decrease in the overall incidence of these three types of HNC in many high-income countries during the last decades, with exception of HPV-driven cancers of the oropharynx.

\section{Course of the disease}

Survival rates are variable and average around 50-60 per cent at 5 years. ${ }^{1,4}$ However, the course of the disease largely depends on the type or anatomic sublocalization of HNC; survival is worse for specific primary sites such as the hypopharynx because of an often advanced stage at diagnosis. Treatment decisions are complex, due to different treatment options including surgery, radiation therapy, chemotherapy, and targeted therapy, and because of the necessity to spare or restore functional and cosmetic aspects. ${ }^{1}$ However, these treatments often have considerable early and late side effects, resulting in significant morbidity with regard to basic functions such as speaking, swallowing, and breathing. In addition, there is a high recurrence risk in HNC, not only at the primary site, but also in the neck and at distance. Furthermore, the prognosis is influenced by the occurrence of second primary tumors, which are the result of so-called field cancerization of the head-neck area. ${ }^{5}$ 
It has been estimated that up to half of these cancers are preventable by associated lifestyle factors and appropriate diets. ${ }^{4}$ Therefore, prevention appears to be a promising strategy in $\mathrm{HNC}$, and insight into possible preventive lifestyle strategies is warranted.

\section{Classification of head-neck cancer}

The most frequently occurring subtypes of HNC are those originating from the oral cavity, pharynx, and larynx. ${ }^{6}$ The pharynx can be further divided into naso-, oro-, and hypopharynx, but nasopharyngeal cancer is a rarity in the Netherlands and other western countries. HNC also includes malignancies from the salivary glands and paranasal sinuses, but these are far less common as well. ${ }^{1}$

HNC can be classified in several ways, depending on a more clinical or epidemiological point of view. From a clinical point of view, the TNM Classification of Malignant Tumours from the International Union Against Cancer (UICC) ${ }^{7}$ is widely used. With regard to epidemiology and etiology, Hashibe et al. ${ }^{8}$ from the International Head and Neck Cancer Epidemiology Consortium (INHANCE) classified HNC in their pooled analyses as oral cavity cancer (OCC), oro-/hypopharyngeal cancer (OHPC), and laryngeal cancer (LC) (Figure 1.1), according to the International Classification of Diseases for Oncology (ICD-O-3). ${ }^{9}$ Both classifications are largely similar, with a classification dividing the oral cavity, pharynx, and larynx. However, in the TNM classification, the oro- and hypopharynx are distinguished and, in addition, the pharynx also includes the nasopharynx. As mentioned before, HNC located in the oral cavity, pharynx, and larynx are the most frequent HNC subtypes, and they share main risk factors.

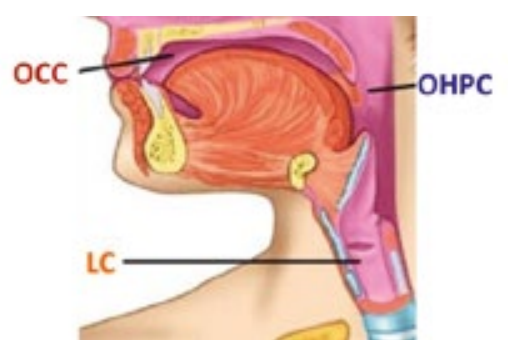

Figure 1.1. Classification of HNC subtypes.

(Adapted image from Google; search terms: 'respiratory system') 


\section{Relevance of head-neck cancer subtypes}

It is relevant to distinguish the head-neck cancer subtypes mentioned above for several reasons. As stated before, treatment strategy and outcome differ per subtype, with generally the worst outcome for oro-/hypopharyngeal cancer. More importantly for the subject of this thesis, the main risk factors for HNC, like smoking, alcohol consumption, and dietary factors, are probably shared by all three subtypes, but the strengths of the associations differ. Therefore, it is relevant to classify HNC into OCC, OHPC, and LC when investigating these factors.

\section{Risk factors for head-neck cancer}

Several factors play a role in the development of HNC, which can be distinguished as demographic, lifestyle and dietary, and other factors.

\section{Demographic factors}

Increasing age and male sex are risk factors for developing HNC, as described above., However, in the last decades, HNC incidence in relatively young adults has been on the rise, whereas the general incidence declined. ${ }^{1,4}$ This rise in HNC incidence in young adults has been linked to HPV infection, which we will discuss later. Regarding sex, $\mathrm{HNC}$ is much more common among men, although this varies by subtype: OCC is also common in women, while OHPC and especially LC mostly occur in men.

\section{Lifestyle and dietary factors}

Alcohol consumption and cigarette smoking are the main risk factors for HNC originating from the oral cavity, pharynx, and larynx, and they are likely to be differentially associated with risk of those HNC subtypes. ${ }^{4,10-14}$ Although cigarette smoking and alcohol consumption are clear risk factors for HNC, there are several remaining questions. It is not precisely clear whether a dose-response relationship ${ }^{8,14}$ and the type of alcohol ${ }^{15}$ - beer, wine or liquor-affect HNC risk, and what differential effect cigarette smoking and alcohol drinking have on HNC subtypes. ${ }^{16}$ This has partly to do with the fact that the majority of conducted studies are case-control studies, a study design susceptible to selection and misclassification with regard to exposure. Prospective cohort studies are less sensitive to this bias, but only six population-based cohort studies have reported on alcohol consumption, cigarette smoking and HNC risk $^{17-23}$, often with a small number of cases and thereby hardly able to examine subtypes. Finally, a greater than multiplicative joint effect between alcohol and 
tobacco consumption has been shown, but most evidence comes from case-control studies as well. ${ }^{17,20-22,24-26}$

Dietary factors probably influence HNC risk as well. In 2007, the World Cancer Research Fund (WCRF) concluded that consumption of non-starchy vegetables and fruits probably protects against HNC originating from the oral cavity, pharynx, and larynx. ${ }^{4}$ However, the evidence was limited and mostly based on case-control studies. Only five population-based cohort studies investigated vegetable and fruit intake and HNC risk. ${ }^{22,27-30}$ Most of them looked at few HNC cases ${ }^{22,28,30}$, did not report on specific HNC subtypes ${ }^{22,28}$ and combined HNC with esophageal ${ }^{22,27,28}$ and gastric ${ }^{30}$ cancer into upper aero-digestive tract cancer (UADTC) for analyses. In addition, adequate adjustment for confounding by smoking is vitally important since smokers are known to consume fewer fruits and vegetables ${ }^{31}$, but the largest prospective study on this topic lacked information on smoking duration, an important aspect of smoking behavior. ${ }^{18,29}$ The WCRF also concluded that foods containing carotenoids are probably protective against HNC, but mostly based on case-control studies, and that evidence regarding other micronutrients like vitamin $C$ and $E$ is scarce. ${ }^{4} A$ protective effect of vitamin $C$ and $E$ is, however, plausible, given their antioxidant capacities. Only one prospective cohort study investigated the association between intake of (vitamins and) carotenoids and the risk on $\mathrm{HNC}^{23}$ Finally, selenium is an essential trace element present in food which may influence carcinogenesis particularly by its antioxidant properties. $^{32}$ In observational studies, selenium status has been associated with a decreased risk of prostate and esophageal cancer and possibly also of lung and stomach cancer. ${ }^{4,33,34}$ A low selenium status may also be associated with an increased risk of $\mathrm{HNC}$, but evidence regarding selenium and $\mathrm{HNC}$ risk is scarce and only based on case-control studies. ${ }^{35-37}$

\section{Other factors}

A low body mass index (BMI) has also been associated with HNC risk, but this association remains to be clarified. Regarding this topic, the WCRF concluded that data regarding the association between body fatness and HNC risk-based on case-control studies, which mostly found inverse associations-were insufficient to allow any conclusions to be drawn. ${ }^{4}$ Three prospective cohort studies investigated the association between $\mathrm{BMI}$ and $\mathrm{HNC}$ risk, but showed mixed results. ${ }^{38-40}$ It remains therefore unclear whether there is a true inverse association between BMI and HNC, or an association due to reverse causality, confounding, or effect modification. ${ }^{4,41-43}$

Finally, human papilloma virus (HPV) infection has been associated with risk of some types of HNC. ${ }^{44-47}$ It is estimated that $30 \%$ of the oropharyngeal cancers are HPV- 
positive and that within this group, the risk is especially increased for tonsil cancer and cancer of the base of the tongue. Risks for other subtypes (oral, other pharyngeal cancers, and laryngeal cancer) are moderately increased. The role of HPV in HNC carcinogenesis is mainly of importance in young HNC patients and has increased since $1990 . .^{47-49}$

\section{Rationale and aim of the thesis}

Since treatment options are limited and survival rates poor, the focus on prevention of HNC-by lifestyle-is of great importance. Given the limited evidence with regard to lifestyle and dietary factors and HNC risk, mainly based on case-control studies, it is crucial to confirm these associations in a prospective cohort study, with comprehensive adjustment for smoking and alcohol consumption. Therefore, the general aim of this thesis was to study and further establish the existing evidence regarding the association between several lifestyle factors and risk of developing HNC and HNC subtypes. We studied the association of 1 ) alcohol consumption and cigarette smoking; 2) consumption of vegetables and fruits; 3 ) intake of vitamins and carotenoids; 4) selenium status; and 5) BMI with HNC overall and HNC subtypes within the large prospective Netherlands Cohort Study (NLCS). We focused on the most frequent HNC subtypes ${ }^{6}$ (those located in the oral cavity, pharynx, and larynx) and hypothesized that 1 ) the risk of HNC is higher in participants with a low intake of vegetables and fruits (and nutrients like carotenoids and vitamins), and those with low levels of toenail selenium; 2) associations of the studied risk factors with risk of HNC are modified by smoking and alcohol consumption; 3) risk of HNC is lower in participants with a high $\mathrm{BMI}$; and that 4) these risks are different when studied for the HNC subtypes OCC, OHPC, and LC.

We believe our large prospective cohort study will add significantly to the current evidence. In addition, we hope to obtain a broader view regarding the development of HNC, with more insight into possible mechanisms regarding factors influencing HNC risk.

\section{Study design}

The NLCS was initiated in September 1986 and includes 120,852 participants, 58,279 men and 62,573 women, aged 55-69 years from 204 Dutch municipal population 
registries. ${ }^{50}$ At baseline, all participants completed a self-administered questionnaire on dietary habits and other cancer risk factors, such as smoking, alcohol consumption, and anthropometry. The 11-page questionnaire included a 150-item food frequency questionnaire that focused on habitual food consumption during the year preceding the start of the study. In addition, about $75 \%$ of the participants collected toenail clippings. The NLCS has been approved by the Medical Ethics Committee of Maastricht University (Maastricht, The Netherlands).

For data processing and analyses, the case-cohort design was used for efficiency in data processing and follow-up. ${ }^{51}$ In this design, cases were derived from the entire cohort, whereas the number of person-years at risk for the entire cohort was estimated using a subcohort of 5,000 people, 2,411 men and 2,589 women. This subcohort was randomly sampled from the total cohort at baseline and followed up for vital status information. The entire cohort is being monitored for cancer incidence by annual record linkage to the Netherlands Cancer Registry (NCR), and the nationwide network and registry of histo- and cytopathology in the Netherlands (PALGA). ${ }^{52}$

Follow-up for vital status of the subcohort was nearly $100 \%$ complete after 20.3 years and the completeness of cancer follow-up is estimated to be $\geq 96 \%$. ${ }^{53}$ Since follow-up for the NLCS is currently ongoing, two different follow-up periods were used in this thesis, respectively 17.3 and 20.3 years. We excluded cohort members who reported having prevalent cancer other than skin cancer at baseline, as well as subjects with missing data on exposure and/or confounding variables. Participants with incomplete or inconsistent dietary data were also excluded from analyses regarding diet and anthropometry. ${ }^{54,55}$ Only microscopically confirmed, first occurrences of squamous cell carcinomas were included. ${ }^{1,4}$ After 20.3 years of follow-up, 415 HNC (131 OCC, 88 OHPC, and 193 LC) cases were available for analysis.

\section{Outline of the thesis}

This thesis starts with the study of alcohol consumption and cigarette smoking with regard to risk of HNC (and HNC subtypes) in our cohort (Chapter 2). In Chapter 3, the association between consumption of vegetables and fruits and the risk of HNC was investigated. Chapter 4 describes the relation between vitamin and carotenoid intake and HNC risk. Furthermore, the associations between toenail selenium status and BMI and HNC were studied in respectively Chapter 5 and 6 . Finally, the findings described in this thesis are generally discussed and put into perspective in Chapter 7. 


\section{References}

1 Argiris, A., Karamouzis, M. V., Raben, D. \& Ferris, R. L. Head and neck cancer. Lancet 371, 1695-1709 (2008).

2 Ferlay J. et al. GLOBOCAN 2008 v1.2, Cancer Incidence and Mortality Worldwide: IARC CancerBase No. 10 [Internet]. Lyon, France: International Agency for Research on Cancer; 2010. Available from: http://globocan.iarc.fr, accessed on 30/05/2013.

3 Parkin, D. M., Bray, F., Ferlay, J. \& Pisani, P. Global cancer statistics, 2002. CA Cancer J Clin 55, 74-108 (2005).

$4 \quad$ World Cancer Research Fund \& American Institute for Cancer Research. Food, Nutrition, Physical Activity and the Prevention of Cancer: a Global Perspective. American Institute for Cancer Research, 2007.

5 Leemans, C. R., Braakhuis, B. J. \& Brakenhoff, R. H. The molecular biology of head and neck cancer. Nat Rev Cancer 11, 9-22 (2011).

6 Dobrossy, L. Epidemiology of head and neck cancer: magnitude of the problem. Cancer Metastasis Rev 24, 9-17 (2005).

7 Sobin, L. H., Gospodarowicz, M. K. \& Wittekind, C. TNM Classification of Malignant Tumours. 7 edn, Geneva: International Union Against Cancer (UICC); Wiley-Blackwell, 2009.

8 Hashibe, M. et al. Alcohol drinking in never users of tobacco, cigarette smoking in never drinkers, and the risk of head and neck cancer: pooled analysis in the International Head and Neck Cancer Epidemiology Consortium. J Nat/ Cancer Inst 99, 777-789 (2007).

9 Fritz, A. G. International Classification of Diseases for Oncology: ICD-O. 3rd edn, World Health Organization, 2000.

10 IARC. Monographs on the evaluation of carcinogenic risks to humans: alcohol drinking. International Agency for Research on Cancer, 1988.

11 IARC. Monographs on the evaluation of carcinogenic risks to humans: tobacco smoke and involuntary smoking. International Agency for Research on Cancer, 2004.

12 Bagnardi, V., Blangiardo, M., La Vecchia, C. \& Corrao, G. Alcohol consumption and the risk of cancer: a meta-analysis. Alcohol Res Health 25, 263-270 (2001).

13 Gandini, S. et al. Tobacco smoking and cancer: a meta-analysis. Int J Cancer 122, 155-164 (2008).

14 Lubin, J. H. et al. Total exposure and exposure rate effects for alcohol and smoking and risk of head and neck cancer: a pooled analysis of case-control studies. Am J Epidemiol 170, 937-947 (2009).

15 Purdue, M. P. et al. Type of alcoholic beverage and risk of head and neck cancer--a pooled analysis within the INHANCE Consortium. Am J Epidemiol 169, 132-142 (2009).

16 Bagnardi, V., Blangiardo, M., La Vecchia, C. \& Corrao, G. A meta-analysis of alcohol drinking and cancer risk. Br J Cancer 85, 1700-1705 (2001).

17 Boeing, H. Alcohol and risk of cancer of the upper gastrointestinal tract: first analysis of the EPIC data. IARC Sci PubI 156, 151-154 (2002).

18 Freedman, N. D., Abnet, C. C., Leitzmann, M. F., Hollenbeck, A. R. \& Schatzkin, A. Prospective investigation of the cigarette smoking-head and neck cancer association by sex. Cancer 110, 1593-1601 (2007).

19 Freedman, N. D., Schatzkin, A., Leitzmann, M. F., Hollenbeck, A. R. \& Abnet, C. C. Alcohol and head and neck cancer risk in a prospective study. Br J Cancer 96, 1469-1474 (2007).

20 Gronbaek, M. et al. Population based cohort study of the association between alcohol intake and cancer of the upper digestive tract. BMJ 317, 844-847 (1998).

21 Kato, I., Nomura, A. M., Stemmermann, G. N. \& Chyou, P. H. Prospective study of the association of alcohol with cancer of the upper aerodigestive tract and other sites. Cancer Causes Control 3, 145-151 (1992).

22 Kjaerheim, K., Gaard, M. \& Andersen, A. The role of alcohol, tobacco, and dietary factors in upper aerogastric tract cancers: a prospective study of 10,900 Norwegian men. Cancer Causes Control 9, 99-108 (1998).

23 Zheng, W. et al. Retinol, antioxidant vitamins, and cancers of the upper digestive tract in a prospective cohort study of postmenopausal women. Am J Epidemiol 142, 955-960 (1995).

24 Hashibe, M. et al. Interaction between tobacco and alcohol use and the risk of head and neck cancer: pooled analysis in the International Head and Neck Cancer Epidemiology Consortium. Cancer Epidemiol Biomarkers Prev 18, 541-550 (2009).

25 Goldstein, B. Y., Chang, S. C., Hashibe, M., La Vecchia, C. \& Zhang, Z. F. Alcohol consumption and cancers of the oral cavity and pharynx from 1988 to 2009: an update. Eur J Cancer Prev 19, 431-465 (2010).

26 IARC. Monographs on the evaluation of carcinogenic risks to humans: personal habits and indoor combustions. International Agency for Research on Cancer, 2012. 
27 Boeing, H. et al. Intake of fruits and vegetables and risk of cancer of the upper aero-digestive tract: the prospective EPIC-study. Cancer Causes Control 17, 957-969 (2006).

28 Chyou, P. H., Nomura, A. M. \& Stemmermann, G. N. Diet, alcohol, smoking and cancer of the upper aerodigestive tract: a prospective study among Hawaii Japanese men. Int J Cancer 60, 616-621 (1995).

29 Freedman, N. D. et al. Fruit and vegetable intake and head and neck cancer risk in a large United States prospective cohort study. Int J Cancer 122, 2330-2336 (2008).

30 Kasum, C. M., Jacobs, D. R., Jr., Nicodemus, K. \& Folsom, A. R. Dietary risk factors for upper aerodigestive tract cancers. Int J Cancer 99, 267-272 (2002).

31 Dallongeville, J., Marecaux, N., Fruchart, J. C. \& Amouyel, P. Cigarette smoking is associated with unhealthy patterns of nutrient intake: a meta-analysis. J Nutr 128, 1450-1457 (1998).

32 Rayman, M. P. Selenium in cancer prevention: a review of the evidence and mechanism of action. The Proceedings of the Nutrition Society 64, 527-542 (2005).

33 Geybels, M. S., Verhage, B. A., van Schooten, F. J., Goldbohm, R. A. \& van den Brandt, P. A. Advanced prostate cancer risk in relation to toenail selenium levels. J Nat/ Cancer Inst 105, 1394-1401 (2013).

34 Steevens, J., van den Brandt, P. A., Goldbohm, R. A. \& Schouten, L. J. Selenium status and the risk of esophageal and gastric cancer subtypes: the Netherlands cohort study. Gastroenterology 138, 1704-1713 (2010).

35 Rogers, M. A. et al. A case-control study of oral cancer and pre-diagnostic concentrations of selenium and zinc in nail tissue. Int J Cancer 48, 182-188 (1991).

36 Knekt, P. et al. Serum micronutrients and risk of cancers of low incidence in Finland. Am J Epidemiol 134, 356-361 (1991).

37 Zheng, W. et al. Serum micronutrients and the subsequent risk of oral and pharyngeal cancer. Cancer Res 53, 795-798 (1993).

38 Hashibe, M. et al. Tobacco, alcohol, body mass index, physical activity, and the risk of head and neck cancer in the prostate, lung, colorectal, and ovarian (PLCO) cohort. Head Neck 35, 914-922 (2013).

39 Gaudet, M. M. et al. Prospective studies of body mass index with head and neck cancer incidence and mortality. Cancer Epidemiol Biomarkers Prev 21, 497-503 (2012).

40 Etemadi, A. et al. A prospective cohort study of body size and risk of head and neck cancers in the NIH-AARP diet and health study. Cancer Epidemiol Biomarkers Prev 23, 2422-2429 (2014).

41 Gaudet, M. M. et al. Body mass index and risk of head and neck cancer in a pooled analysis of case-control studies in the International Head and Neck Cancer Epidemiology (INHANCE) Consortium. Int J Epidemiol 39, 1091-1102 (2010).

42 Lubin, J. H. et al. Body mass index, cigarette smoking, and alcohol consumption and cancers of the oral cavity, pharynx, and larynx: modeling odds ratios in pooled case-control data. Am J Epidemiol 171, 1250-1261 (2010).

43 Lubin, J. H. et al. An examination of male and female odds ratios by BMI, cigarette smoking, and alcohol consumption for cancers of the oral cavity, pharynx, and larynx in pooled data from 15 case-control studies. Cancer Causes Control 22, 1217-1231 (2011).

44 Hobbs, C. G. et al. Human papillomavirus and head and neck cancer: a systematic review and meta-analysis. Clin Otolaryngol 31, 259-266 (2006).

45 IARC. A Review of Human Carcinogens: Biological Agents. Vol. 100B International Agency for Research on Cancer, 2012.

46 Marur, S., D'Souza, G., Westra, W. H. \& Forastiere, A. A. HPV-associated head and neck cancer: a virus-related cancer epidemic. Lancet Oncol 11, 781-789 (2010).

47 Mehanna, H. et al. Prevalence of human papillomavirus in oropharyngeal and nonoropharyngeal head and neck cancer--systematic review and meta-analysis of trends by time and region. Head Neck 35, 747-755 (2013).

48 Curado, M. P. \& Hashibe, M. Recent changes in the epidemiology of head and neck cancer. Curr Opin Oncol 21, 194200 (2009).

49 Marur, S. \& Forastiere, A. A. Head and neck cancer: changing epidemiology, diagnosis, and treatment. Mayo Clin Proc 83, 489-501 (2008).

50 van den Brandt, P. A. et al. A large-scale prospective cohort study on diet and cancer in The Netherlands. J Clin Epidemiol 43, 285-295 (1990).

51 Barlow, W. E., Ichikawa, L., Rosner, D. \& Izumi, S. Analysis of case-cohort designs. J Clin Epidemiol 52, 1165-1172 (1999).

52 van den Brandt, P. A., Schouten, L. J., Goldbohm, R. A., Dorant, E. \& Hunen, P. M. Development of a record linkage protocol for use in the Dutch Cancer Registry for Epidemiological Research. Int J Epidemiol 19, 553-558 (1990). 
53 Goldbohm RA, Van den Brandt PA \& Dorant E. Estimation of the coverage of Dutch municipalities by cancer registries and PALGA based on hospital discharge data. Tijdschr Soc Gezondheidsz, 80-84 (1994).

54 Goldbohm, R. A. et al. Validation of a dietary questionnaire used in a large-scale prospective cohort study on diet and cancer. Eur J Clin Nutr 48, 253-265 (1994).

55 Heinen, M. M., Verhage, B. A., Goldbohm, R. A. \& van den Brandt, P. A. Intake of vegetables, fruits, carotenoids and vitamins $C$ and $E$ and pancreatic cancer risk in The Netherlands Cohort Study. Int J Cancer 130, 147-158 (2012). 
Chapter 2

Alcohol consumption, cigarette smoking and the risk of subtypes of head-neck cancer: results from the Netherlands Cohort Study

\author{
Denise H. E. Maasland \\ Piet A. van den Brandt \\ Bernd Kremer \\ R. Alexandra Goldbohm \\ Leo J. Schouten
}




\section{Abstract}

\section{Background}

Prospective data on alcohol consumption, cigarette smoking and risk of head-neck cancer (HNC) subtypes, i.e., oral cavity cancer (OCC), oro-/hypopharyngeal cancer (OHPC), and laryngeal cancer (LC), are limited. We investigated these associations within the second largest prospective study on this topic so far, the Netherlands Cohort Study.

\section{Methods}

120,852 participants completed a questionnaire on diet and other cancer risk factors in 1986. After 17.3 years of follow-up, 395 HNC (110 OCC, 83 OHPC, and 199 LC) cases and 4,288 subcohort members were available for case-cohort analysis using Cox proportional hazards models.

\section{Results}

For total HNC, the multivariable-adjusted incidence rate ratio (RR) was 2.74 (95\% confidence interval ( $\mathrm{Cl}$ ) 1.85-4.06) for those drinking $\geq 30 \mathrm{~g}$ ethanol/day compared with abstainers; in subtypes, RRs were 6.39 for OCC, 3.52 for OHPC, and 1.54 for LC. Compared with never cigarette smokers, current cigarette smokers had a RR of 4.49 (95\% Cl 3.11-6.48) for HNC overall, and 2.11 for OCC, 8.53 for OHPC, and 8.07 for LC. A significant, positive, multiplicative interaction between categories of alcohol consumption and cigarette smoking was found for HNC overall ( $P$ interaction $=0.03$ ).

\section{Conclusions}

Alcohol consumption and cigarette smoking were independently associated with risk of HNC overall, with a positive, multiplicative interaction. The strength of these associations differed among HNC subtypes: OCC was most strongly associated with alcohol consumption but most weakly with cigarette smoking, whereas LC was not statistically significantly associated with alcohol consumption. 


\section{Introduction}

Head and neck cancer (HNC) includes several malignancies that originate in the paranasal sinuses, nasal cavity, salivary glands, oral cavity, pharynx, and larynx. ${ }^{1} \mathrm{HNC}$ is the seventh most common type of cancer in the world and in the European Union; in Europe, HNC accounts for an estimated 130,000 new cases every year. ${ }^{2}$

Alcohol consumption and cigarette smoking are established risk factors for HNC originating from the oral cavity, pharynx, and larynx, and are likely to be differentially associated with risk of those HNC subtypes. ${ }^{3-8}$ However, the majority of conducted studies are case-control studies, a study design susceptible to misclassification with regard to exposure. Prospective cohort studies are less sensitive to this bias, but only six population-based cohort studies have reported on alcohol consumption, cigarette smoking and HNC risk. ${ }^{9-15}$ Of these studies, most had a small number of cases and were thereby hardly able to examine subtypes; HNC was often combined with other cancers into upper aerodigestive tract cancer. ${ }^{9,12-15}$ In addition, the largest prospective study so far lacked information on smoking duration. ${ }^{10}$ Finally, a greater than multiplicative joint effect between alcohol and tobacco consumption has been shown, but most evidence comes from case-control studies as well. ${ }^{9,12-14,16-18}$

Therefore, we wanted to investigate these associations in HNC subtypes within the large prospective Netherlands Cohort Study (NLCS). We focused on the most frequent HNC subtypes: those located in the oral cavity, pharynx, and larynx, and hypothesized that 1) alcohol consumption and cigarette smoking are strongly, positively associated with HNC risk, with multiplicative interaction; and that 2) these risks are different for oral cavity cancer (OCC), oro-/hypopharyngeal cancer (OHPC), and laryngeal cancer (LC).

\section{Methods}

\section{Study design and population}

The present study was conducted within the NLCS, which started in September 1986 with the inclusion of 120,852 participants, aged 55-69 years from 204 Dutch municipal population registries. ${ }^{19}$

For data processing and analysis, the case-cohort design was used for reasons of efficiency. ${ }^{20}$ Cases were derived from the total cohort, whereas the number of personyears at risk for the total cohort was estimated from a subcohort of 5,000 persons, randomly sampled from the entire cohort at baseline. 
Follow-up for cancer incidence was done by annual record linkage to the Netherlands Cancer Registry and the nationwide network and pathology registry. ${ }^{21}$ The completeness of cancer follow-up is estimated to be $\geq 96 \%{ }^{22}$, and follow-up for vital status of the subcohort was nearly $100 \%$ complete after 17.3 years.

We excluded cohort members who reported to have prevalent cancer other than skin cancer at baseline, and cases and subcohort members with missing data on exposure or confounding variables. Only microscopically confirmed, first occurrences of squamous cell carcinomas-which include nearly all malignancies of the mouth, pharynx, and larynx $x^{1,3}$ - of the head and neck were included.

In total, 395 incident HNC cases and 4288 subcohort members were available for analysis (Figure 2.1). Of these cases, 110 were oral cavity cancer (ICD-O-3 C003-009, C020-C023, C030-C031, C039-C041, C048-C050, C060-C062, C068-C069), 83 oro/hypopharyngeal cancer (C019, C024, C051-C052, C090-C091, C098-C104, C108-C109, C129-C132, C138-C139); 3 oral cavity, pharynx unspecified, or overlapping (C028-C029, C058-C059, C140-C142, C148), and 199 laryngeal cancer (C320-C329) cases, classified as proposed by Hashibe et al. $^{23}$, according to the International Classification of Diseases for Oncology (ICD-O-3). ${ }^{24}$

The NLCS has been approved by the Medical Ethics Committee of Maastricht University (Maastricht, The Netherlands).

\begin{tabular}{|c|c|c|c|c|}
\hline \multicolumn{5}{|c|}{ Netherlands Cohort Study on diet and cancer $(120,852)$} \\
\hline$\downarrow$ & & & $\downarrow$ & \\
\hline $\begin{array}{l}\text { Subcohort, randomly } \\
\text { sampled from total cohort }\end{array}$ & \multicolumn{4}{|c|}{ Record linkage with Netherlands Cancer Registry and PALGA } \\
\hline$\stackrel{\downarrow}{\downarrow}$ & $\begin{array}{l}\downarrow \\
\text { HNC overall }^{\mathrm{a}}\end{array}$ & $\begin{array}{l}\stackrel{\downarrow}{\text { Oral cavity }} \\
\text { cancer (OCC) }\end{array}$ & $\begin{array}{l}\qquad \\
\qquad \\
\text { Oro-/hypopharyngeal } \\
\text { cancer (OHPC) }\end{array}$ & $\begin{array}{l}\downarrow \\
\text { Laryngeal } \\
\text { cancer (LC) }\end{array}$ \\
\hline \multicolumn{5}{|c|}{ Exclusion of participants with prevalent cancer at baseline } \\
\hline $\begin{array}{c}\downarrow \\
4,774\end{array}$ & $\underset{451}{\downarrow}$ & $\begin{array}{c}\downarrow \\
119\end{array}$ & $\begin{array}{l}\downarrow \\
99\end{array}$ & $\underset{230}{\downarrow}$ \\
\hline \multicolumn{5}{|c|}{ Exclusion of participants with missing data on alcohol consumption or cigarette smoking } \\
\hline $\begin{array}{c}\downarrow \\
4,288\end{array}$ & $\underset{395}{\downarrow}$ & $\underset{110}{\downarrow}$ & $\begin{array}{l}\downarrow \\
83\end{array}$ & $\begin{array}{c}\downarrow \\
199\end{array}$ \\
\hline
\end{tabular}

Figure 2.1. Flow diagram of the number of subcohort members and cases on whom the analyses were based. Abbreviation PALGA: nationwide network and registry of histopathology and cytopathology in the Netherlands. ${ }^{a}$ Oral cavity cancer; oro-/hypopharyngeal cancer; oral cavity, pharynx unspecified or overlapping cancer; laryngeal cancer. 


\section{Exposure data}

At baseline, all cohort members completed a self-administered questionnaire, which included a 150-item food frequency questionnaire (FFQ) with detailed questions on alcohol consumption, smoking habits, and other cancer risk factors.

We asked about the habitual intake of alcohol during the year preceding the start of the study, measured by six items: 1) beer; 2) red wine; 3) white wine; 4) sherry and other fortified wines; 5) liquor types containing on average $16 \%$ alcohol; and 6) (Dutch) gin, brandy, and whisky. In addition, questions were asked about the frequency of consumption and the number of glasses consumed on each drinking occasion. For analysis, we combined 2), 3), and 4) into 'wine', and 5) and 6) into 'liquor'. Total mean daily ethanol intake was calculated using the Dutch food-composition table. ${ }^{25}$ On the basis of pilot study data, standard glass sizes were defined as $200 \mathrm{~mL}$ for beer, $105 \mathrm{~mL}$ for wine, and $45 \mathrm{~mL}$ for liquor, corresponding to 8,10 , and 13 grams of ethanol, respectively. ${ }^{26}$ We also asked questions about the consumption of 'beer' and 'other alcoholic beverages' 5 years before baseline and selected participants with stable alcohol consumption to perform a sensitivity analysis. ${ }^{27}$ Participants who indicated that they used alcoholic beverages never or less than once a month were considered abstainers.

We asked detailed information regarding cigarette smoking. Among others, questions were asked about whether the subject was a smoker at baseline; age at which they started and stopped smoking; the number of cigarettes smoked daily and the number of smoking years (excluding stopping periods). Based on these questions, the following variables were constructed for analysis: smoking status (never/former/current); current smoking (yes/no); frequency (cigarettes/day); duration (years); the number of pack-years; and time since smoking cessation (years). We also asked about cigar and pipe smoking and the use of smokeless tobacco. Participants who indicated they had never smoked cigarettes were considered never smokers.

The FFQ was validated against a 9-day diet record, and the Spearman correlation coefficient between the alcohol intake assessed by the questionnaire and that estimated by the diet record was 0.89 for all subjects and 0.85 for users of alcoholic beverages. $^{28}$ The reproducibility of the FFQ was assessed through annually repeated measurements in a subgroup of the subcohort and the test-retest correlation was 0.90 for alcohol intake; this correlation declined only 0.01-0.02 per year. ${ }^{29}$

Data were key-entered and processed in a standardized manner, blinded with regard to case/subcohort status in order to minimize observer bias in coding and data interpretation. 


\section{Statistical analysis}

Person-years at risk were calculated from baseline until diagnosis of HNC, death, emigration, loss to follow-up, or end of follow-up (i.e., 31 December 2003), whichever occurred first.

Age (years) and sex were considered predefined confounders. The potential confounders considered were ${ }^{3,30,31}$ : level of education; non-occupational physical activity; energy intake; coffee and tea consumption; intake of fruit, vegetables, fish, fat, red meat, and meat products; and family history of head-neck cancer. Alcohol consumption and cigarette smoking were mutually adjusted in statistical models. A variable was considered a confounder if including it in the model changed the incidence rate ratio (RR) for HNC overall or any of the HNC subtypes by $>10 \%$; according to this, none of the potential confounders was included in the final model.

The Cox proportional hazards model was used to estimate incidence RRs and corresponding 95\% confidence intervals $(\mathrm{Cl})$ for alcohol consumption and cigarette smoking in multivariable-adjusted case-cohort analyses. Analyses were done using the Stata 11.2 statistical software package (StataCorp, College Station, Texas, USA). Standard errors were calculated using the robust Huber-White sandwich estimator to account for additional variance introduced by sampling from the cohort; this method is equivalent to the variance-covariance estimator by Barlow. ${ }^{32}$ The proportional hazards assumption was assessed using the scaled Schoenfeld residuals. ${ }^{33}$ If there was an indication for violation of the assumption for a variable, we further investigated this by adding a time-varying covariate for that variable to the model.

We also analyzed beer, wine, and liquor consumption, adjusted for ethanol intake, to examine whether substances in alcoholic beverages, other than ethanol, have an effect on HNC risk. In smoking analyses, different aspects of cigarette smoking were investigated and mutually adjusted for, in order to obtain a complete exposure model. The total number of cases that exclusively smoked cigar and/or pipe or used smokeless tobacco was too low $(N<10)$ to further analyze associations with HNC risk.

When adjusting for smoking frequency, duration, or pack-years, we centered these continuous variables as proposed by Leffondré et al. ${ }^{34}$

Tests for linear dose-response trends were assessed by fitting ordinal exposure variables as continuous terms. To evaluate possible multiplicative interaction between categories of alcohol consumption and cigarette smoking, we estimated RRs of HNC overall and all HNC subtypes for combinations of these exposures. The interaction was investigated by including cross-product terms in the model and performing a Wald test. Two-sided $P$ values are reported throughout the article. 
Tests for heterogeneity among HNC subtypes were performed to investigate differences in associations among HNC subtypes by a bootstrapping method developed for the case-cohort design. ${ }^{35}$ For each bootstrap sample, $X$ subcohort members were randomly drawn from the subcohort of $X$ subjects and $Y$ cases from the total of $Y$ cases outside the subcohort, both with replacement, out of the dataset of $X+Y$ observations. The logRRs were obtained from this sample using Stata's competing risks procedure and recalculated for each bootstrap-replication. The confidence interval and $P$ value of the differences in incidence rate ratio of the subtypes were then calculated from the replicated statistics. Each bootstrap analysis was based on at least 1,000 replications. ${ }^{36}$

\section{Results}

Compared to the subcohort, cases were more frequently men than women, and less often alcohol abstainers (Table 2.1). Among alcohol consumers, cases had a substantially higher alcohol intake and generally drank more beer, wine, and liquor than subcohort members. In both cases and subcohort members, men mostly consumed beer and liquor, whereas women drank more wine. With respect to cigarette smoking, cases were far more often current smokers and also smoked a substantially higher number of pack-years than subcohort members. Women were more often never smokers than men; among ever smokers, men generally smoked more pack-years than women, in cases and subcohort members.

\section{Alcohol consumption}

Alcohol consumption of $\geq 30$ grams (g) per day compared with abstinence was associated with a statistically significantly increased risk of HNC overall (multivariableadjusted RR: $2.74,95 \% \mathrm{Cl}$ 1.85-4.06), OCC (RR: 6.39, 95\% Cl 3.13-13.03), and OHPC (RR: 3.52, 95\% Cl 1.69-7.36), but not LC (RR: 1.54, 95\% Cl 0.91-2.60) (Table 2.2). A strong dose-response relationship ( $P$ trend<0.001) was found between categories of increasing alcohol consumption and HNC overall, OCC, and OHPC risk. A significant interaction was found between sex and continuous alcohol consumption in HNC overall ( $P=0.02)$ and OCC ( $P=0.004)$, with women having higher RRs than men.

After adjustment for total alcohol intake, consumption of beer, wine, and liquor was generally not significantly associated with HNC risk. Beer consumption was, however, statistically significantly, positively associated with OHPC-risk ( $P$ trend $=0.03$ ); liquor consumption was significantly associated with an increased risk of OCC 


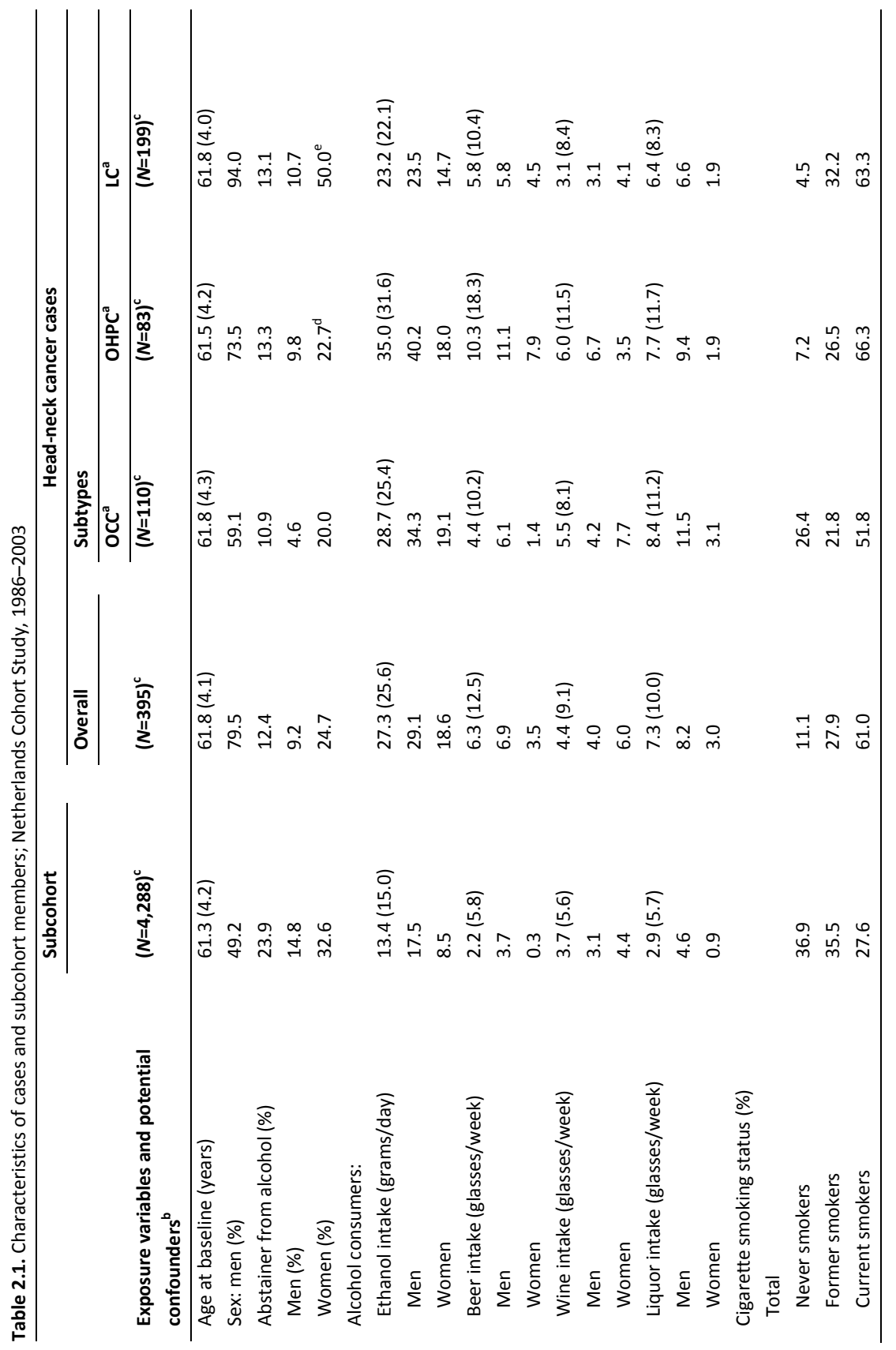




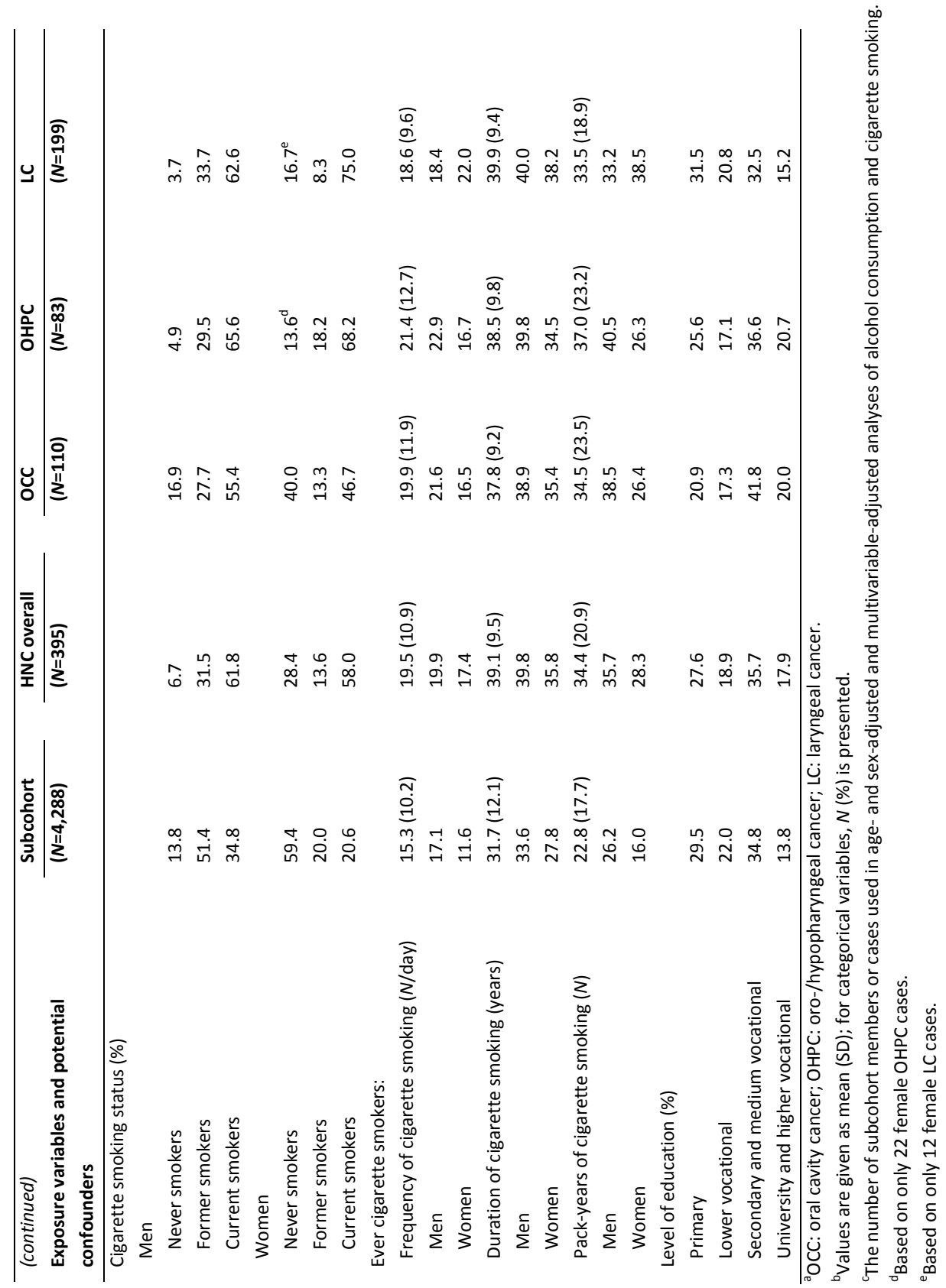




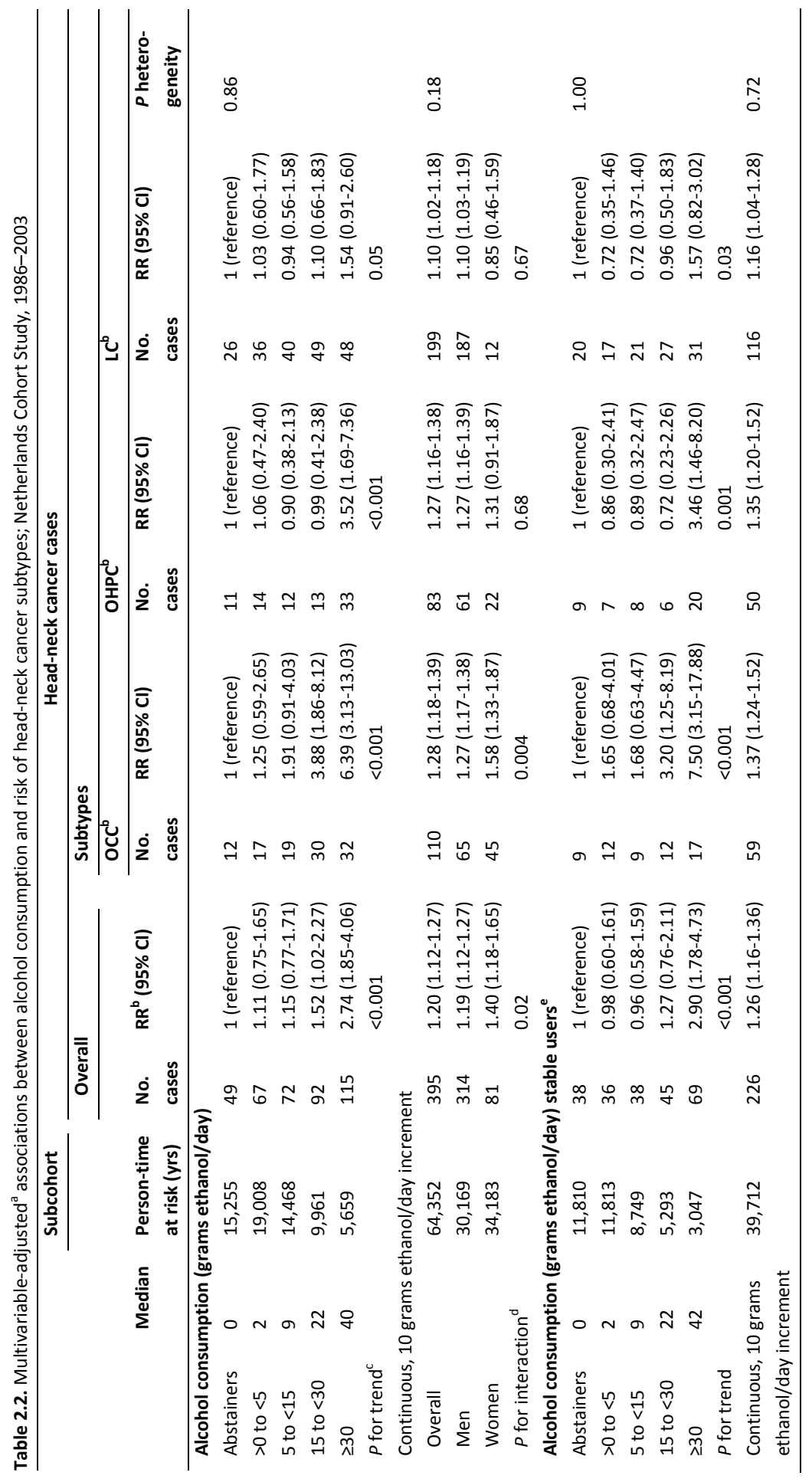




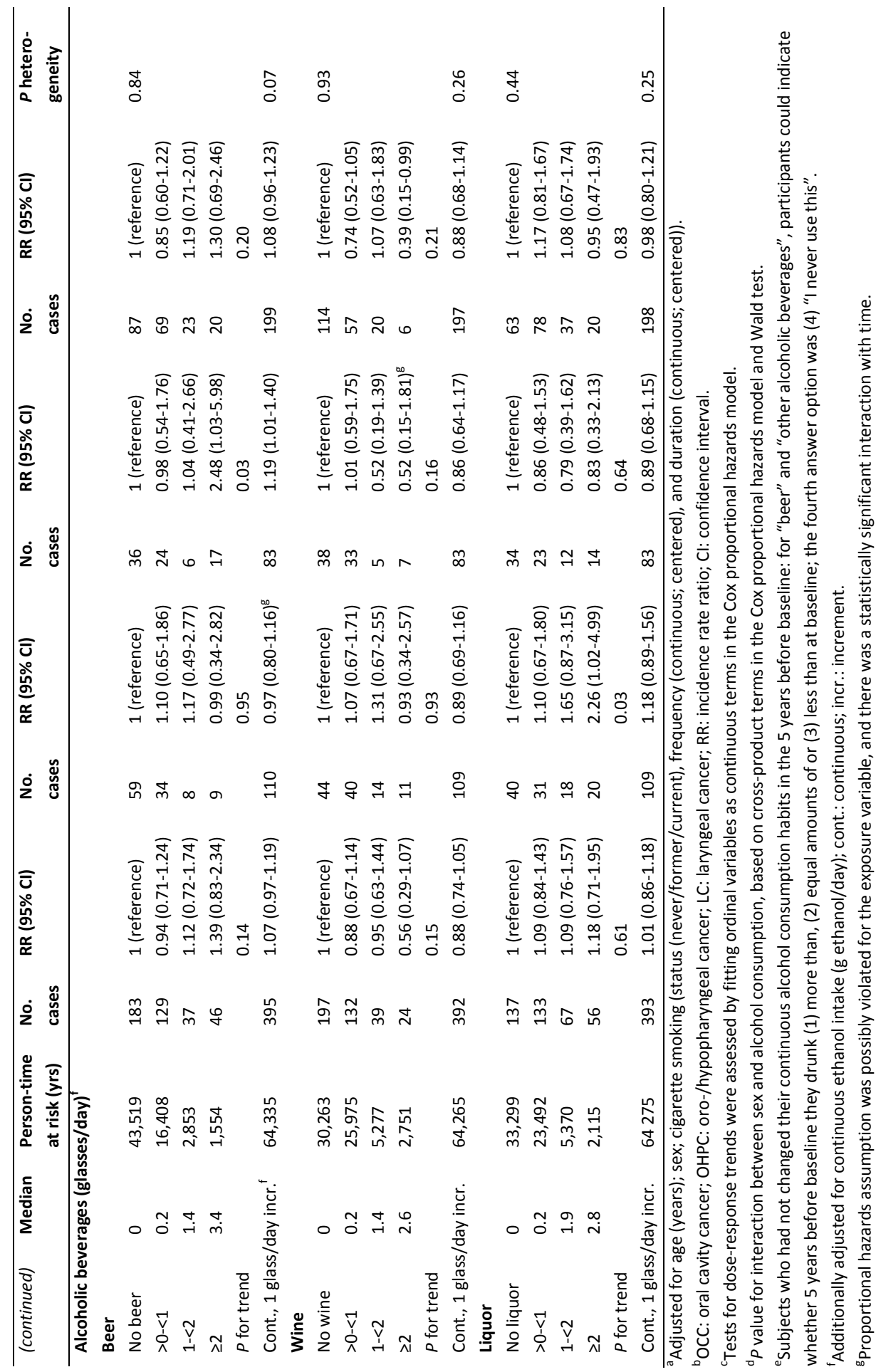




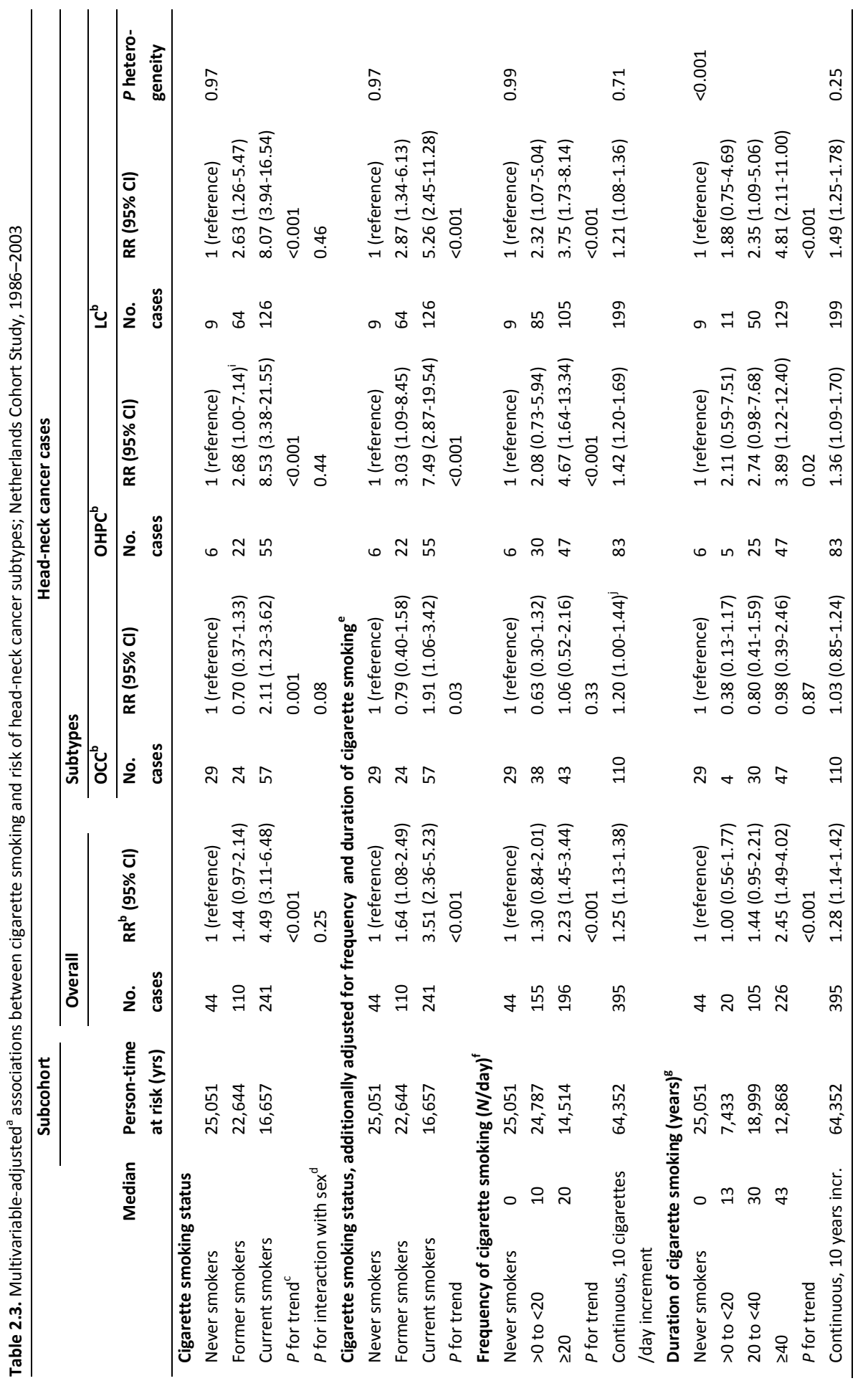




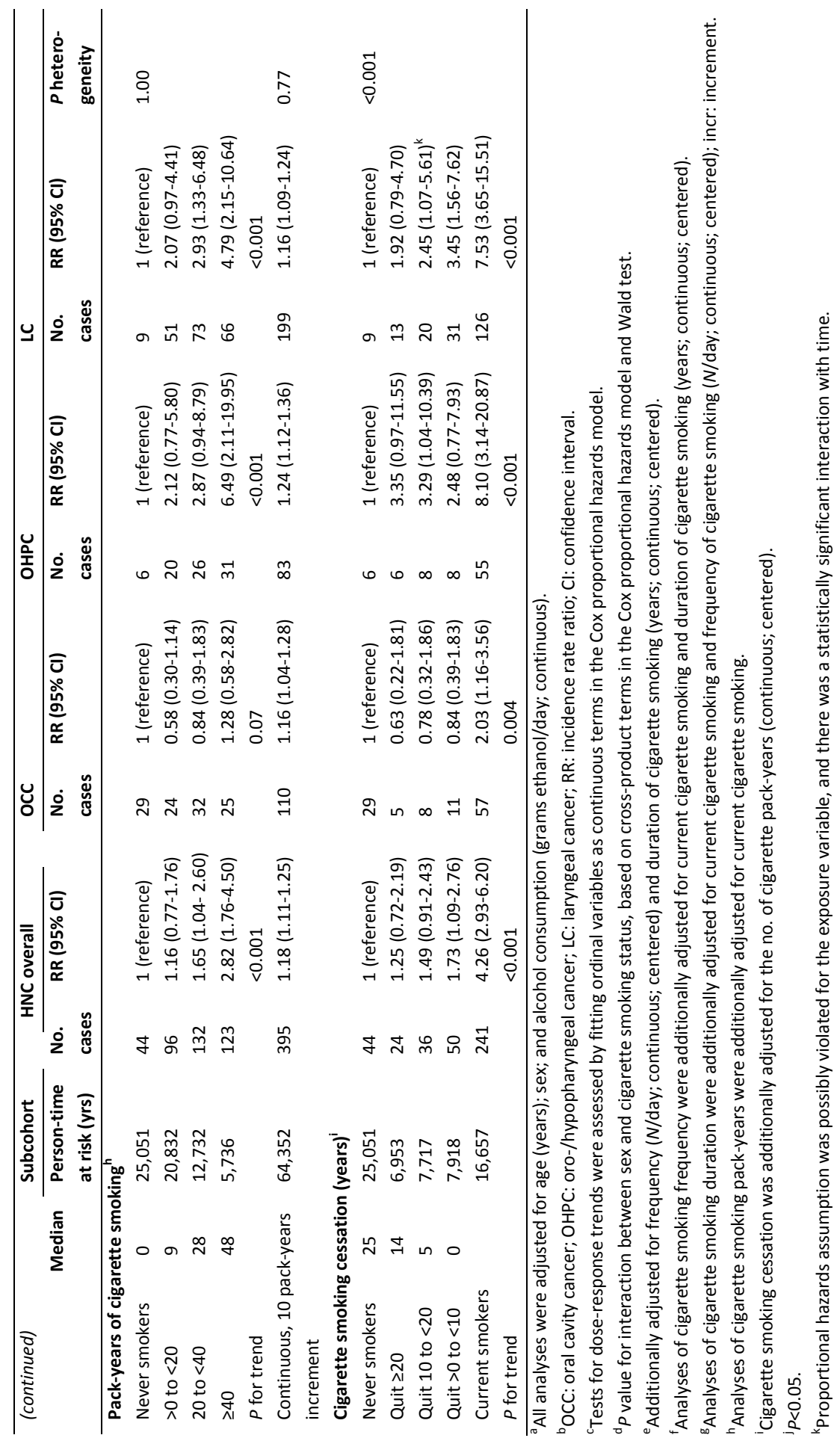




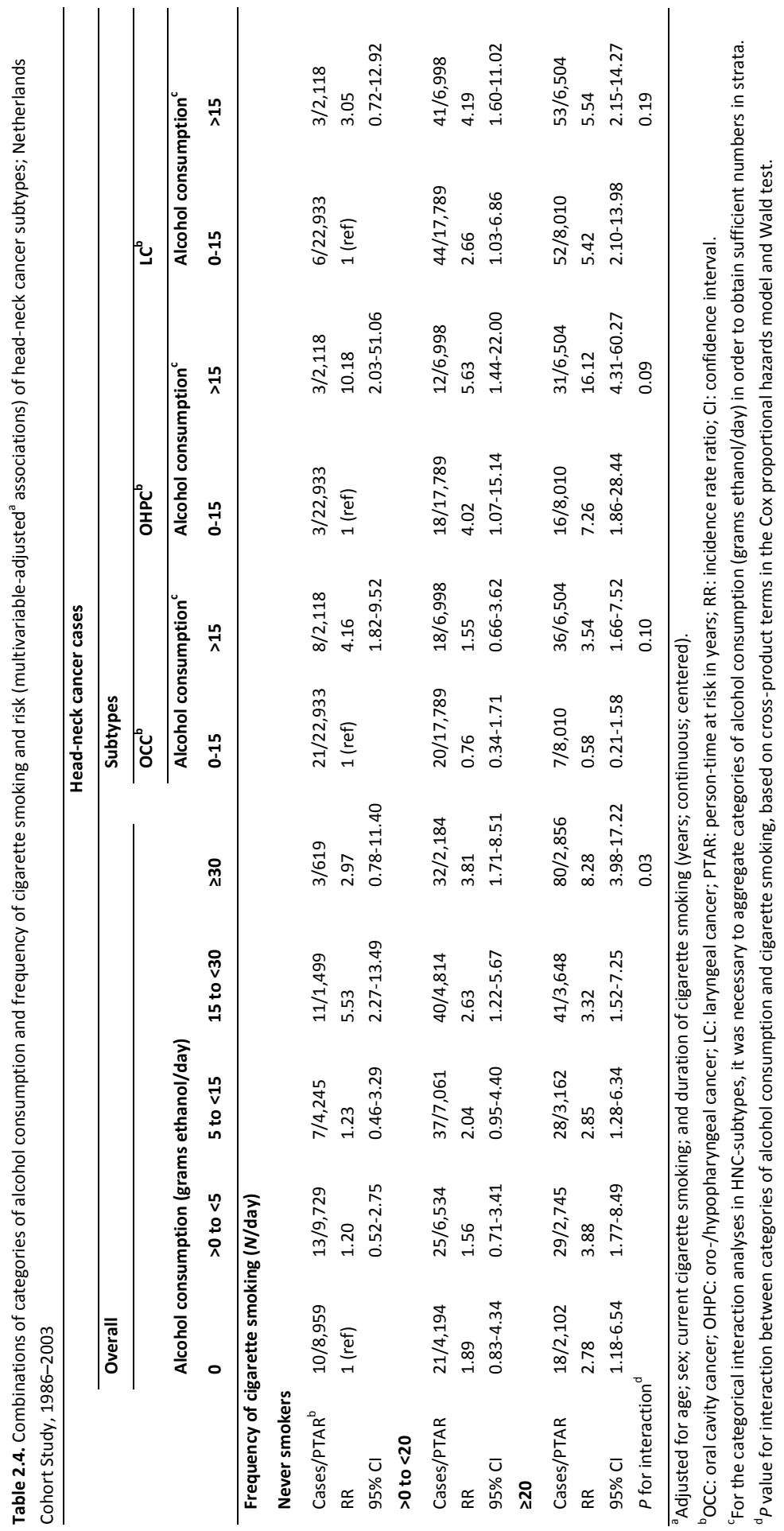


( $P$ trend=0.03). Wine consumption was largely inversely associated-although not statistically significantly-with risk of HNC overall and HNC subtypes.

Although RRs clearly varied among HNC subtypes, tests for heterogeneity did not show any significant differences in associations, possibly due to low power.

\section{Cigarette smoking}

Current cigarette smoking was statistically significantly associated with risk of HNC overall (multivariable-adjusted RR: $4.49,95 \% \mathrm{Cl} 3.11-6.48$ ) and all subtypes, with strongest associations in OHPC (RR: $8.53,95 \% \mathrm{Cl} 3.38-21.55)$ and LC (RR: $8.07,95 \% \mathrm{Cl}$ 3.94-16.54), compared with never smoking (Table 2.3). Compared with never smoking, former cigarette smoking was also associated with risk of HNC overall, although not statistically significantly (RR: $1.44,95 \% \mathrm{Cl} 0.97-2.14$ ), OHPC (RR: $2.68,95 \% \mathrm{Cl} 1.00-$ 7.14), and LC (RR: $2.63,95 \% \mathrm{Cl} 1.26-5.47$ ), but not OCC (RR: $0.70,95 \% \mathrm{Cl} 0.37-1.33$ ). Frequency and duration of cigarette smoking were also strongly, statistically significantly associated with an increased risk of HNC overall, OHPC, and LC (Table 2.3).

Regarding different aspects of cigarette smoking, after mutual adjustment, cigarette smoking status, frequency, and duration all remained statistically significantly associated with risk of HNC overall, OHPC, and LC (see Supplemental Tables 2.1 and 2.2). After additional adjustment for alcohol consumption (Table 2.3), most RRs between cigarette smoking status, frequency, duration and risk of HNC (subtypes) slightly attenuated, but remained statistically significantly associated with increased risks.

Results regarding smoking cessation show that the risk of HNC overall and all subtypes diminished for smokers who stopped smoking since $<10,10$ to $<20$, or $\geq 20$ years, compared with current smokers (all $P$ trend<0.01) (Table 2.3). Nevertheless, compared with never smokers, RRs 20 years after smoking cessation were still elevated for HNC overall, OHPC, and LC, although not statistically significantly.

Despite considerable differences in RRs among HNC subtypes, tests for heterogeneity only showed statistically significant differences in associations for duration of cigarette smoking $(P<0.001)$ and time since smoking cessation $(P<0.001)$.

\section{Interaction between alcohol consumption and cigarette smoking}

For HNC overall, increased risks were found for every exposure combination of alcohol consumption and cigarette smoking, mostly statistically significantly, compared to never smokers and abstainers as reference group (Table 2.4). In addition, a statistically significant, positive, multiplicative interaction was found ( $P$ interaction $=0.03$ ) between categories of alcohol consumption and cigarette smoking, with a RR of $8.28(95 \% \mathrm{Cl}$ 
3.98-17.22), comparing participants smoking $\geq 20$ cigarettes and drinking $\geq 30 \mathrm{~g}$ alcohol per day with never smokers abstaining from alcohol.

In HNC subtypes, RRs were mostly increased as well when comparing participants smoking $\geq 20$ cigarettes and drinking $>15 \mathrm{~g}$ alcohol per day with never smokers consuming 0 to $15 \mathrm{~g}$ alcohol per day, with the highest RR for OHPC (RR: $16.12,95 \% \mathrm{Cl}$ 4.31-60.27), but no significant interaction was found, possibly due to low numbers in strata.

\section{Discussion}

In this large prospective study on alcohol consumption, cigarette smoking, and risk of HNC (subtypes), alcohol consumption and cigarette smoking were strongly, independently associated with an increased risk of HNC overall. The strength of these associations however differed between HNC subtypes; OCC was most strongly associated with alcohol consumption but most weakly with cigarette smoking, whereas LC was not statistically significantly associated with alcohol consumption. For HNC overall, a multiplicative interaction between categories of alcohol consumption and cigarette smoking was found.

\section{Alcohol consumption}

Our results are in agreement with those of previous studies, showing alcohol consumption to be an independent risk factor for the development of HNC, with a strong, dose-response relationship., 4,9,11-14,17,23,37,38 Alcoholic beverages and acetaldehyde, the main metabolite of ethanol, are classified as a class I carcinogen. ${ }^{18}$ It is plausible that alcohol-after being metabolized-acts both directly and indirectly in HNC carcinogenesis, the latter for example by acting as a solvent for other possible carcinogens, such as tobacco carcinogens. ${ }^{3,39}$

The differential risk among HNC subtypes is consistent with other studies, in which LC was also least associated with alcohol consumption. ${ }^{8,40,41}$ However, several other studies found OHPC being most associated with alcohol consumption, although sometimes in specific subgroups, as opposed to OCC in our study. ${ }^{11,23,41}$ Nevertheless, the differential risk among HNC subtypes is likely to be explained by the larynx having the least direct exposure to alcohol compared with the oral cavity and pharynx. ${ }^{39,42}$ The slightly increased RRs for alcohol consumption and LC may be due to inhalation of alcohol containing aerosols, silent aspiration, systemic effects, and possibly residual confounding. 
After adjustment for total alcohol intake, we generally found similar risks between intake of beer, wine, liquor and HNC. These findings imply that ethanol itself probably is the most important factor in determining HNC risk, rather than other substances in alcoholic beverages, which is in line with the results from other studies. ${ }^{3,11,42}$ Consumption of wine was, however, generally inversely associated with HNC risk, as was also shown in a pooled analysis ${ }^{42}$, which could be due to residual confounding by a general healthier lifestyle of wine-consumers in our study population.,42,43

The significantly higher RRs between alcohol consumption and HNC risk in women as compared with men were seen earlier and could possibly be explained by women having stronger carcinogenic effects of alcohol at the same exposure level, suggesting possible gender-specific risk or protective factors. ${ }^{11}$

\section{Cigarette smoking}

This study confirms the strong associations of cigarette smoking with increased risk of HNC overall and all subtypes. ${ }^{3,5,7,10,14,23,37,41}$ Among subtypes, however, OCC was least associated with cigarette smoking, and strongest associations were found with OHPC and LC. In addition, smoking status, frequency, and duration all appear to be of importance in the association between cigarette smoking and risk of HNC overall, OHPC, and LC. These results are generally consistent with previous reviews showing that cigarette smoking has a stronger effect on the larynx and/or pharynx than on the oral cavity ${ }^{7,8,10,23,41}$; in two meta-analyses, the larynx seemed to be clearly most susceptible to the effects of cigarette smoking. ${ }^{23,41}$ A possible explanation for this could be the aerodynamics of respiratory flow in the upper airway: this flow changes from laminar in the oral cavity to turbulent in the larynx, which may result in the larynx and pharynx having a higher exposure to inhaled air-and thus to cigarette smoke-than the oral cavity.

Finally, our study shows smoking cessation leads to decreased HNC risks, which is in line with results from a recent pooled analysis as well. ${ }^{44}$

\section{Interaction between alcohol consumption and cigarette smoking}

Our study confirms a multiplicative interaction between categories of alcohol consumption and cigarette smoking in HNC overall. ${ }^{9,14,16-18,37,38,41}$ The interaction effect between alcohol consumption and cigarette smoking is biologically plausible, since alcohol can act as a solvent for carcinogens in cigarette smoke and make the mucosa more permeable for these carcinogens; as a result, the carcinogenic properties of both factors are likely to be enhanced in the presence of one another. ${ }^{3,39}$ Still, in HNC 
subtypes, we had low numbers of cases in strata, which probably resulted in limited power to detect a significant deviation from the multiplicative model.

\section{Strengths and limitations}

Important strengths of our study are the prospective character and completeness of follow-up. Our study is the second largest prospective cohort study investigating alcohol consumption and cigarette smoking on the risk of HNC overall and subtypes so far. ${ }^{9-15}$ Furthermore, we were able to take into account data on smoking duration, and to investigate as well as adjust for several aspects of smoking behavior.

A possible limitation of our study is the single measurement of exposure data. Alcohol consumption and cigarette smoking were however extensively addressed in the questionnaire, with questions about lifetime exposure history of smoking and alcohol intake 5 years before baseline. It is however possible that participants who smoked at baseline in 1986 stopped smoking at some point during follow-up or changed their alcohol intake, and this may have led to bias due to misclassification. Furthermore, although our study includes a large number of cases, a lack of power is a possible explanation for finding non-significant results for some associations and the tests for heterogeneity.

We lack information on human papillomavirus (HPV) infection. HPV infection is associated with $\mathrm{HNC}$ risk $^{45,46}$, but mainly with $\mathrm{OHPC}$, in particular tonsil cancer and cancer of the base of the tongue. According to rates in our university medical center, only $25 \%$ of the diagnosed and treated oropharyngeal cancers between 1997 and 2003 were HPV-positive (all oropharyngeal cancer cases have been analyzed by p16immunostaining and HPV16-specific fluorescence in situ hybridization (FISH), and-if FISH was negative-HPV-specific polymerase chain reaction). Moreover, the role of HPV in HNC carcinogenesis is mainly of importance in young HNC patients, and has increased since $1990 .^{47-49}$ Since our participants were aged 55-69 years at baseline in 1986, we assume that the number of HPV-associated HNC cases in our cohort is low, and we expect potential bias due to possible misclassification to be very limited.

Other factors we did not take into account in our analyses are the use of drugs and oral hygiene. Although we investigated several potential confounders, residual confounding is still possible, but we presume this to be limited as well.

It might also be interesting to examine the RRs of HNC for smokers among nondrinkers and for drinkers among non-smokers. However, as the case numbers for these subgroups would be too small to analyze, we decided not to investigate this.

Finally, though we wanted to examine the role of alcohol consumption and cigarette smoking in HNC subtypes, we did not investigate HNC located in the major 
salivary glands, nasal cavity, paranasal sinuses, and nasopharynx, because of low numbers of these cases as well as a presumably different etiology. ${ }^{50}$

\section{Conclusions}

In conclusion, the present study, which is the second largest prospective cohort study regarding this topic so far, confirms the principal role of alcohol consumption and cigarette smoking in HNC carcinogenesis, as well as the differential associations with HNC subtypes, and a significant, positive, multiplicative interaction between both factors. As the existing evidence is largely based on case-control studies, this cohort study contributes to establish in which extent alcohol consumption and cigarette smoking are associated with risk of HNC overall and, more specifically, HNC subtypes.

\section{References}

1 Argiris, A., Karamouzis, M. V., Raben, D. \& Ferris, R. L. Head and neck cancer. Lancet 371, 1695-1709 (2008).

2 Ferlay J. et al. GLOBOCAN 2008 v1.2, Cancer Incidence and Mortality Worldwide: IARC CancerBase No. 10 [Internet]. Lyon, France: International Agency for Research on Cancer; 2010. Available from: http://globocan.iarc.fr, accessed on $30 / 05 / 2013$

3 World Cancer Research Fund \& American Institute for Cancer Research. Food, Nutrition, Physical Activity and the Prevention of Cancer: a Global Perspective. American Institute for Cancer Research, 2007.

4 IARC. Monographs on the evaluation of carcinogenic risks to humans: alcohol drinking. International Agency for Research on Cancer, 1988.

5 IARC. Monographs on the evaluation of carcinogenic risks to humans: tobacco smoke and involuntary smoking. International Agency for Research on Cancer, 2004.

6 Bagnardi, V., Blangiardo, M., La Vecchia, C. \& Corrao, G. Alcohol consumption and the risk of cancer: a meta-analysis. Alcohol Res Health 25, 263-270 (2001).

7 Gandini, S. et al. Tobacco smoking and cancer: a meta-analysis. Int J Cancer 122, 155-164 (2008).

8 Lubin, J. H. et al. Total exposure and exposure rate effects for alcohol and smoking and risk of head and neck cancer: a pooled analysis of case-control studies. Am J Epidemiol 170, 937-947 (2009).

9 Boeing, $\mathrm{H}$. Alcohol and risk of cancer of the upper gastrointestinal tract: first analysis of the EPIC data. IARC Sci PubI 156, 151-154 (2002).

10 Freedman, N. D., Abnet, C. C., Leitzmann, M. F., Hollenbeck, A. R. \& Schatzkin, A. Prospective investigation of the cigarette smoking-head and neck cancer association by sex. Cancer 110, 1593-1601 (2007).

11 Freedman, N. D., Schatzkin, A., Leitzmann, M. F., Hollenbeck, A. R. \& Abnet, C. C. Alcohol and head and neck cancer risk in a prospective study. Br J Cancer 96, 1469-1474 (2007).

12 Gronbaek, M. et al. Population based cohort study of the association between alcohol intake and cancer of the upper digestive tract. BMJ 317, 844-847 (1998).

13 Kato, I., Nomura, A. M., Stemmermann, G. N. \& Chyou, P. H. Prospective study of the association of alcohol with cancer of the upper aerodigestive tract and other sites. Cancer Causes Control 3, 145-151 (1992).

14 Kjaerheim, K., Gaard, M. \& Andersen, A. The role of alcohol, tobacco, and dietary factors in upper aerogastric tract cancers: a prospective study of 10,900 Norwegian men. Cancer Causes Contro/ 9, 99-108 (1998)

15 Zheng, W. et al. Retinol, antioxidant vitamins, and cancers of the upper digestive tract in a prospective cohort study of postmenopausal women. Am J Epidemiol 142, 955-960 (1995).

16 Hashibe, M. et al. Interaction between tobacco and alcohol use and the risk of head and neck cancer: pooled analysis in the International Head and Neck Cancer Epidemiology Consortium. Cancer Epidemiol Biomarkers Prev 18, 541-550 (2009). 
17 Goldstein, B. Y., Chang, S. C., Hashibe, M., La Vecchia, C. \& Zhang, Z. F. Alcohol consumption and cancers of the oral cavity and pharynx from 1988 to 2009: an update. Eur J Cancer Prev 19, 431-465 (2010).

18 IARC. Monographs on the evaluation of carcinogenic risks to humans: personal habits and indoor combustions. International Agency for Research on Cancer, 2012.

19 van den Brandt, P. A. et al. A large-scale prospective cohort study on diet and cancer in The Netherlands. J Clin Epidemiol 43, 285-295 (1990).

20 Barlow, W. E., Ichikawa, L., Rosner, D. \& Izumi, S. Analysis of case-cohort designs. J Clin Epidemiol 52, 1165-1172 (1999).

21 van den Brandt, P. A., Schouten, L. J., Goldbohm, R. A., Dorant, E. \& Hunen, P. M. Development of a record linkage protocol for use in the Dutch Cancer Registry for Epidemiological Research. Int J Epidemiol 19, 553-558 (1990).

22 Goldbohm RA, Van den Brandt PA \& Dorant E. Estimation of the coverage of Dutch municipalities by cancer registries and PALGA based on hospital discharge data. Tijdschr Soc Gezondheidsz, 80-84 (1994).

23 Hashibe, M. et al. Alcohol drinking in never users of tobacco, cigarette smoking in never drinkers, and the risk of head and neck cancer: pooled analysis in the International Head and Neck Cancer Epidemiology Consortium. J Natl Cancer Inst 99, 777-789 (2007).

24 Fritz, A. G. International Classification of Diseases for Oncology: ICD-O. 3rd edn, World Health Organization, 2000.

25 NEVO table: Dutch food composition table, 1986-1987. The Hague, the Netherlands: Voorlichtingbureau Voor de Voeding, 1986.

26 Brants, H. A. M. \& den Breeijen, H. Beschrijving en verantwoording van de codering van NEVO-codes, standaardporties e.d. (Description of and justification for coding of the NEVO-codes, standardized serving sizes, etc. [in Dutch]). Zeist, the Netherlands: Institution CIVO - Toxicology and Nutrition TNO, 1990

27 Bongaerts, B. W., van den Brandt, P. A., Goldbohm, R. A., de Goeij, A. F. \& Weijenberg, M. P. Alcohol consumption, type of alcoholic beverage and risk of colorectal cancer at specific subsites. Int J Cancer 123, 2411-2417 (2008).

28 Goldbohm, R. A. et al. Validation of a dietary questionnaire used in a large-scale prospective cohort study on diet and cancer. Eur J Clin Nutr 48, 253-265 (1994).

29 Goldbohm, R. A. et al. Reproducibility of a food frequency questionnaire and stability of dietary habits determined from five annually repeated measurements. Eur J Clin Nutr 49, 420-429 (1995).

30 Negri, E. et al. Family history of cancer: pooled analysis in the International Head and Neck Cancer Epidemiology Consortium. Int J Cancer 124, 394-401 (2009).

31 Galeone, C. et al. Coffee and tea intake and risk of head and neck cancer: pooled analysis in the international head and neck cancer epidemiology consortium. Cancer Epidemiol Biomarkers Prev 19, 1723-1736 (2010).

32 Barlow, W. E. Robust variance estimation for the case-cohort design. Biometrics 50, 1064-1072 (1994).

33 Schoenfeld, D. Partial residuals for the proportional hazards regression model. Biometrika 69, 239-241 (1982).

34 Leffondre, K., Abrahamowicz, M., Siemiatycki, J. \& Rachet, B. Modeling smoking history: a comparison of different approaches. Am J Epidemiol 156, 813-823 (2002).

35 Wacholder, S., Gail, M. H., Pee, D. \& Brookmeyer, R. Alternative Variance and Efficiency Calculations for the CaseCohort Design. Biometrika 76, 117-123 (1989).

36 de Vogel, S. et al. Dietary folate, methionine, riboflavin, and vitamin B-6 and risk of sporadic colorectal cancer. J Nutr 138, 2372-2378 (2008).

37 Pelucchi, C., Gallus, S., Garavello, W., Bosetti, C. \& La Vecchia, C. Alcohol and tobacco use, and cancer risk for upper aerodigestive tract and liver. Eur J Cancer Prev 17, 340-344 (2008).

38 Chyou, P. H., Nomura, A. M. \& Stemmermann, G. N. Diet, alcohol, smoking and cancer of the upper aerodigestive tract: a prospective study among Hawaii Japanese men. Int J Cancer 60, 616-621 (1995).

39 Boffetta, P. \& Hashibe, M. Alcohol and cancer. Lancet Oncol 7, 149-156 (2006).

40 Bagnardi, V., Blangiardo, M., La Vecchia, C. \& Corrao, G. A meta-analysis of alcohol drinking and cancer risk. Br J Cancer 85, 1700-1705 (2001).

41 Zeka, A., Gore, R. \& Kriebel, D. Effects of alcohol and tobacco on aerodigestive cancer risks: a meta-regression analysis. Cancer Causes Control 14, 897-906 (2003).

42 Purdue, M. P. et al. Type of alcoholic beverage and risk of head and neck cancer--a pooled analysis within the INHANCE Consortium. Am J Epidemiol 169, 132-142 (2009).

43 Balder, H. F., Goldbohm, R. A. \& van den Brandt, P. A. Dietary patterns associated with male lung cancer risk in the Netherlands Cohort Study. Cancer Epidemiol Biomarkers Prev 14, 483-490 (2005).

44 Marron, M. et al. Cessation of alcohol drinking, tobacco smoking and the reversal of head and neck cancer risk. Int J Epidemiol 39, 182-196 (2010). 
45 D'Souza, G. et al. Case-control study of human papillomavirus and oropharyngeal cancer. N Engl J Med 356, 1944-1956 (2007).

46 Hobbs, C. G. et al. Human papillomavirus and head and neck cancer: a systematic review and meta-analysis. Clin Otolaryngol 31, 259-266 (2006).

47 Curado, M. P. \& Hashibe, M. Recent changes in the epidemiology of head and neck cancer. Curr Opin Oncol 21, 194200 (2009).

48 Marur, S. \& Forastiere, A. A. Head and neck cancer: changing epidemiology, diagnosis, and treatment. Mayo Clin Proc 83, 489-501 (2008).

49 Mehanna, H. et al. Prevalence of human papillomavirus in oropharyngeal and nonoropharyngeal head and neck cancer--systematic review and meta-analysis of trends by time and region. Head Neck 35, 747-755 (2013).

50 Dobrossy, L. Epidemiology of head and neck cancer: magnitude of the problem. Cancer Metastasis Rev 24, 9-17 (2005). 


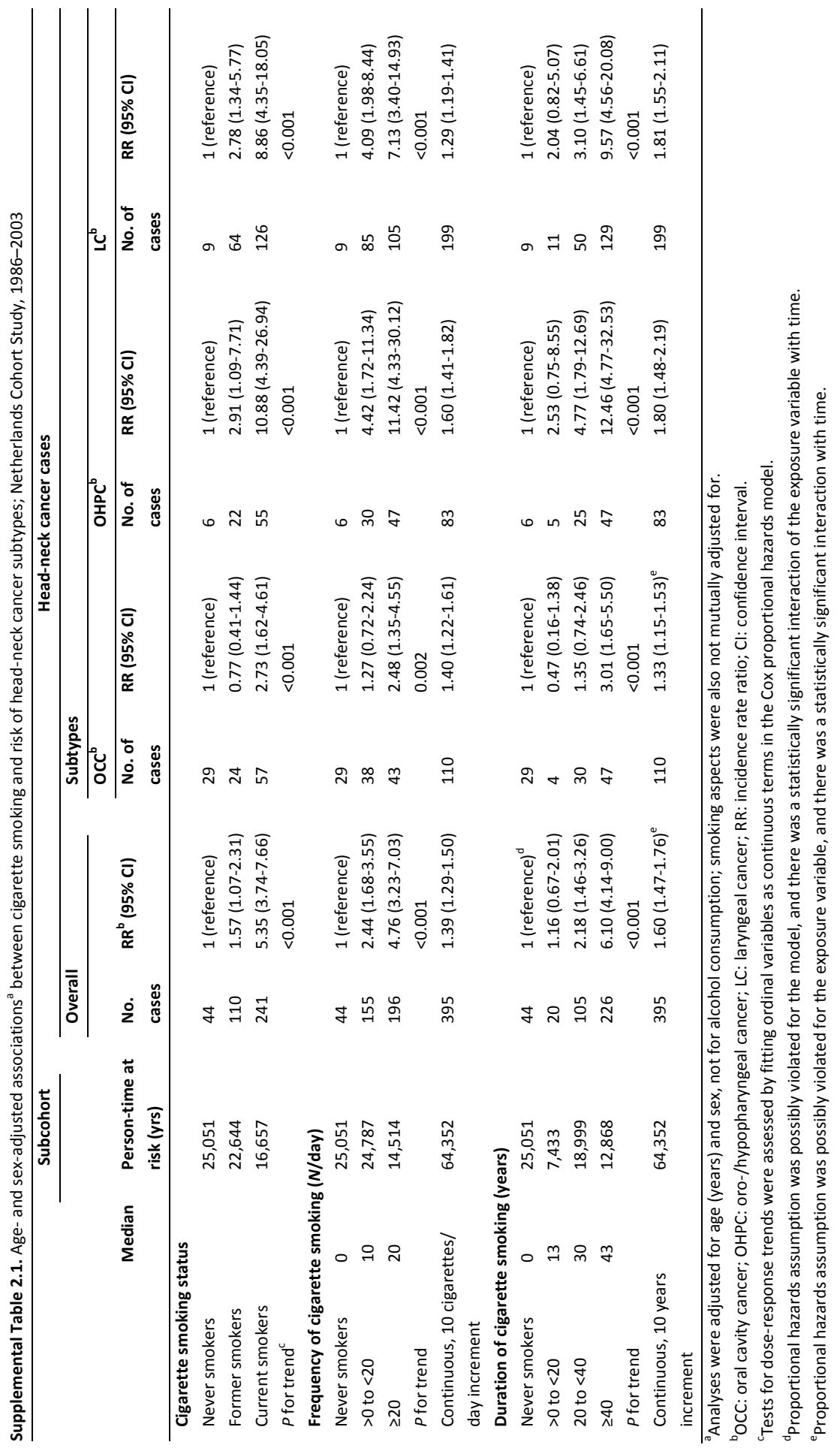




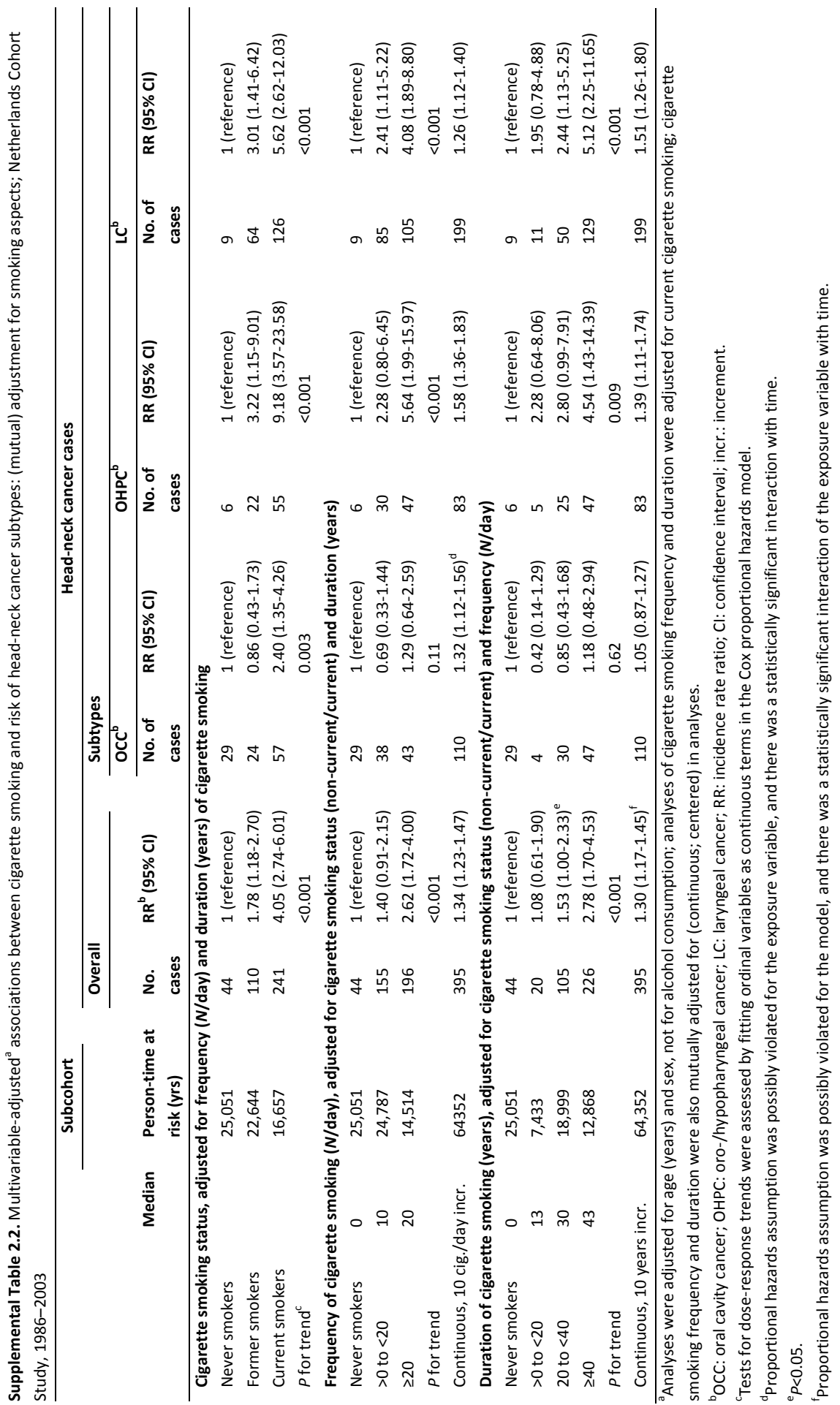


Chapter 3

Consumption of vegetables and fruits and risk of subtypes of head-neck cancer in the Netherlands Cohort Study

Denise H. E. Maasland Piet A. van den Brandt

Bernd Kremer

R. Alexandra Goldbohm Leo J. Schouten 


\begin{abstract}
Background

There is limited prospective data on the relationship between consumption of vegetables and fruits and the risk of head-neck cancer (HNC) subtypes (i.e., oral cavity cancer (OCC), oro-/hypopharyngeal cancer (OHPC), and laryngeal cancer (LC)). Therefore, we investigated these associations within the Netherlands Cohort Study.

\section{Methods}

120,852 participants completed a 150 -item food frequency questionnaire at baseline in 1986. After 20.3 years of follow-up, 415 cases of HNC (131 OCC, 88 OHPC, 3 oral cavity/pharynx unspecified or overlapping, and 193 LC) and 3,898 subcohort members were available for case-cohort analysis using Cox proportional hazards models.

\title{
Results
}

Total vegetable and fruit consumption was inversely associated with risk of HNC overall (multivariable-adjusted incidence rate ratio for highest vs. lowest quartile: 0.61, 95\% confidence interval $(\mathrm{Cl}) 0.44-0.85, P$ trend=0.002) and all HNC subtypes, with the strongest associations for OCC. Total vegetable intake and total fruit intake were also associated with a decreased risk of HNC overall and HNC subtypes. No significant interaction was found between vegetable and fruit intake and alcohol consumption or cigarette smoking.

\section{Conclusions}

In conclusion, in this large-scale cohort study, consumption of vegetables and fruits was associated with a decreased risk of HNC overall and all subtypes. Consumption of vegetables and fruits (or of specific groups of them) may protect against HNC and its subtypes. 


\section{Introduction}

Head and neck cancer (HNC) is the seventh most common type of cancer in the world and Europe, and includes, among others, malignancies originating from the oral cavity, pharynx, and larynx. ${ }^{1,2}$ Alcohol consumption, cigarette smoking and human papillomavirus (HPV) infection are established risk factors for $\mathrm{HNC}^{3,4}$ However, intake of vegetables and fruits has been consistently associated with a decreased risk of HNC. $^{3}$ Vegetables and fruits contain numerous substances, such as carotenoids and flavonoids, that may exert anticarcinogenic properties. ${ }^{5}$

In 2007, the World Cancer Research Fund concluded that consumption of nonstarchy vegetables and fruits probably protects against HNC originating from the oral cavity, pharynx, and larynx. ${ }^{3}$ However, the evidence was limited and is still mainly based on case-control studies, which are susceptible to bias with regard to exposure. In addition, adequate adjustment for confounding by smoking is vitally important since smokers are known to consume fewer fruits and vegetables. ${ }^{6}$ For these reasons, it is crucial to confirm the inverse association between vegetable and fruit consumption and HNC risk in prospective cohort studies with comprehensive adjustment for smoking.

Only five population-based cohort studies have investigated vegetable and fruit intake and HNC risk. $^{7-11}$ Most of them looked at few HNC cases ${ }^{8,10,11}$, did not report on specific HNC subtypes ${ }^{8,11}$ (or only in very small numbers ${ }^{10}$ ), and combined HNC with esophageal $^{7,8,11}$ and gastric ${ }^{10}$ cancer into upper aero-digestive tract cancer (UADTC) for analyses. Furthermore, the largest prospective study on this topic lacked information on smoking duration, an important aspect of smoking behavior. ${ }^{9,12}$ Two prospective studies investigated possible effect modification by smoking and alcohol consumption on the link between vegetable and fruit intake and HNC risk, but they found no differential risk among strata. ${ }^{7,9}$

Therefore, we aimed to investigate the association between consumption of vegetables and fruits and $\mathrm{HNC/HNC}$ subtypes within the large prospective Netherlands Cohort Study (NLCS). We focused on the most frequent HNC subtypes ${ }^{13}$ (those located in the oral cavity, pharynx, and larynx) and hypothesized that 1) the risk of HNC is higher in participants with low intake of vegetables and fruits; and that 2) these risks are different for oral cavity cancer (OCC), oro-/hypopharyngeal cancer (OHPC), and laryngeal cancer (LC). In addition, we will investigate the association of vegetables and fruits with HNC risk according to smoking status and categories of alcohol consumption. 


\section{Methods}

\section{Study design and population}

The present study was conducted within the NLCS, which was initiated in September 1986 and includes 120,852 participants, aged 55-69 years from 204 Dutch municipal population registries. ${ }^{14}$ At baseline, all participants completed a self-administered questionnaire about diet, lifestyle habits and other cancer risk factors. The NLCS has been approved by the Medical Ethics Committee of Maastricht University (Maastricht, The Netherlands).

We used the case-cohort design for efficiency in data processing and follow-up. ${ }^{15}$ Cases were derived from the entire cohort. In contrast, the number of person-years at risk for the entire cohort was estimated using a subcohort of 5,000 people who were randomly sampled from the total cohort at baseline.

Follow-up for cancer incidence was done by record linkage to the Netherlands Cancer Registry (NCR) and the nationwide network and pathology registry (PALGA). ${ }^{16}$ Follow-up for vital status of the subcohort was nearly $100 \%$ complete after 20.3 years and the completeness of cancer follow-up is estimated to be $\geq 96 \%{ }^{17}$

We excluded cohort members who reported having prevalent cancer other than skin cancer at baseline (Figure 3.1). Participants with incomplete or inconsistent dietary data and/or inconsistent vegetable data were also excluded from analysis. ${ }^{18,19}$ Finally, subjects with missing data on confounding variables (300 subcohort members and 40 cases) were excluded. Only microscopically confirmed, first occurrences of squamous cell carcinomas-which include nearly all malignancies of the mouth, pharynx, and larynx-were included. ${ }^{1,3}$

In total, 3,898 subcohort members and 415 incident cases of the selected HNC subtypes were available for analysis (Figure 3.1). Of these cases, 131 were oral cavity cancer (ICD-O-3 C003-009, C020-C023, C030-C031, C039-C041, C048-C050, C060-C062, C068-C069), 88 were oro-/hypopharyngeal cancer (C019, C024, C051-C052, C090-C091, C098-C104, C108-C109, C129-C132, C138-C139), 3 were oral cavity/pharynx unspecified or overlapping (C028-C029, C058-C059, C140-C142, C148), and 193 were laryngeal (C320-C329) cancer cases. They were classified as proposed by Hashibe et al. $^{20}$ according to the International Classification of Diseases for Oncology (ICD-O-3). ${ }^{21}$

\section{Questionnaire data}

The questionnaire included a 150-item food frequency questionnaire (FFQ) that focused on habitual food consumption during the year preceding the start of the study. The FFQ covered most vegetables and fruits eaten regularly in 1986, with the 


\begin{tabular}{|c|c|c|c|c|}
\hline \multicolumn{5}{|c|}{ Netherlands Cohort Study on diet and cancer $(120,852)$} \\
\hline$\downarrow$ & & & $\downarrow$ & \\
\hline $\begin{array}{l}\text { Subcohort, randomly } \\
\text { sampled from total cohort }\end{array}$ & \multicolumn{4}{|c|}{ Record linkage with Netherlands Cancer Registry and PALGA } \\
\hline $\begin{array}{c}\downarrow \\
5,000\end{array}$ & $\begin{array}{l}\downarrow \\
\text { HNC overall }^{a}\end{array}$ & $\begin{array}{l}\qquad \\
\qquad \\
\text { Oral cavity } \\
\text { cancer (OCC) }\end{array}$ & $\begin{array}{l}\qquad \\
\qquad \\
\text { Oro-/hypopharyngeal } \\
\text { cancer (OHPC) }\end{array}$ & $\begin{array}{l}\downarrow \\
\downarrow \\
\text { Laryngeal } \\
\text { cancer (LC) }\end{array}$ \\
\hline \multicolumn{5}{|c|}{ Exclusion of participants with prevalent cancer at baseline } \\
\hline $\begin{array}{c}\downarrow \\
4,774\end{array}$ & $\begin{array}{c}\downarrow \\
515\end{array}$ & $\begin{array}{c}\downarrow \\
149\end{array}$ & $\begin{array}{c}\downarrow \\
109\end{array}$ & $\begin{array}{c}\downarrow \\
254\end{array}$ \\
\hline \multicolumn{5}{|c|}{ Exclusion of participants with incomplete or inconsistent dietary data } \\
\hline $\begin{array}{c}\downarrow \\
4,439\end{array}$ & $\underset{473}{\downarrow}$ & $\begin{array}{c}\downarrow \\
140\end{array}$ & $\begin{array}{c}\downarrow \\
102\end{array}$ & $\begin{array}{c}\downarrow \\
228\end{array}$ \\
\hline \multicolumn{5}{|c|}{ Exclusion of participants with inconsistent vegetable data } \\
\hline $\begin{array}{c}\downarrow \\
4,198\end{array}$ & $\underset{455}{\downarrow}$ & $\begin{array}{c}\downarrow \\
136\end{array}$ & $\begin{array}{l}\downarrow \\
98\end{array}$ & $\begin{array}{c}\downarrow \\
218\end{array}$ \\
\hline \multicolumn{5}{|c|}{ Exclusion of participants with missing data on confounders } \\
\hline $\begin{array}{c}\downarrow \\
3,898\end{array}$ & $\begin{array}{c}\downarrow \\
415\end{array}$ & $\begin{array}{c}\downarrow \\
131\end{array}$ & $\begin{array}{l}\downarrow \\
88\end{array}$ & $\begin{array}{c}\downarrow \\
193\end{array}$ \\
\hline
\end{tabular}

Figure 3.1. Flow diagram of the number of subcohort members and cases on whom the analyses were based. Abbreviation PALGA: nationwide network and registry of histopathology and cytopathology in the Netherlands. ${ }^{a}$ Oral cavity cancer; oro-/hypopharyngeal cancer; oral cavity, pharynx unspecified or overlapping cancer; laryngeal cancer.

exception of chicory, cucumber and red cabbage. Broccoli was rarely available in the Netherlands in 1986 and was therefore not included in the FFQ.

In addition, we asked detailed questions about alcohol consumption and cigarette smoking. We asked about the habitual intake of alcohol during the year preceding the start of the study, measuring six items: 1) beer; 2) red wine; 3) white wine; 4) sherry and other fortified wines; 5) types of liquor that contained $16 \%$ alcohol on average; and 6) a category that included (Dutch) gin, brandy and whisky. In addition, we asked about the frequency of consumption and the number of glasses consumed on each drinking occasion. We also asked about the consumption of 'beer' and 'other alcoholic beverages' five years before baseline. Participants who indicated that they used alcoholic beverages never or less than once a month were considered to be abstainers. We also asked detailed questions about cigarette smoking, including whether the subject was a smoker at baseline, the age at which they started and stopped smoking, the number of cigarettes smoked daily, and the number of smoking years (excluding stopping periods).

Data were key-entered and processed in a standardized manner, blinded with respect to case/subcohort status in order to minimize observer bias in coding and data 
interpretation. In order to maintain blindness of data entry during follow-up, we selected an additional subcohort (from the total baseline cohort).

All subjects were asked to report their frequency of consumption of a number of vegetables (for summer and winter separately) and fruits (Table 3.1). Consumption frequency was specified by using categories ranging from 'never or less than once per month' to '3-7 times per week' for vegetable intake and '6-7 times per week' for fruit consumption. For individual fruit items, we also asked about the amount consumed per serving. In an open-ended question, participants could indicate which other vegetables and fruits they consumed on a regular basis, together with the consumption frequency (number of times per week) and amount consumed per serving. For onions and tomatoes, subjects were asked to report their usual intake in number per week; for sweet peppers, per month; and for mushrooms, the number of 250-gram boxes per month.

Participants were only asked about their usual serving size for string beans and cooked endive. Based on results of a pilot study, the mean of these serving sizes was used as a representative for the serving sizes of all solid and leafy vegetables, respectively. This individual serving size was then multiplied with a vegetable-specific factor to derive an individual serving size for each vegetable. To calculate mean daily vegetable consumption (grams/day) and the intake of individual fruit items (grams/day), we used consumption frequency and serving sizes.

Table 3.1. Composition of vegetable and fruit groups, based on vegetable and fruit items that were asked in the food frequency questionnaire

\begin{tabular}{|c|c|}
\hline Food group & Composition \\
\hline Total vegetables & Cooked vegetables plus raw vegetables \\
\hline Cooked vegetables & $\begin{array}{l}\text { Beetroot, broad beans, Brussels sprouts, cauliflower, cabbage (white/green), cooked } \\
\text { carrots, cooked endive, kale, leek, mushrooms, onions, rhubarb, sauerkraut, spinach, } \\
\text { string beans, sweet peppers, and other cooked vegetables originating from an open- } \\
\text { ended question on frequently consumed items not listed in the questionnaire }\end{array}$ \\
\hline Brassica vegetables & Brussels sprouts, cabbage (white/green), cauliflower and kale \\
\hline Leafy vegetables, cooked & Cooked endive and spinach \\
\hline Leafy vegetables, raw & Lettuce and raw endive \\
\hline Citrus fruits & $\begin{array}{l}\text { Fresh lemon juice, grapefruits and fresh grapefruit juice, mandarins, and oranges and } \\
\text { fresh orange juice }\end{array}$ \\
\hline
\end{tabular}


The FFQ was validated against a nine-day diet record. The Spearman correlation coefficient was 0.38 for total vegetable consumption and 0.60 for total fruit intake. ${ }^{18}$ On average, vegetable consumption appeared to be slightly overestimated by the FFQ when compared to the diet records, whereas fruit consumption seemed to be underestimated.

The reproducibility of the FFQ was assessed by annual repeated measurements in a subgroup of the subcohort; the average test-retest correlation over all nutrients was 0.66 and ranged from 0.42 to $0.90 .^{22}$ The average decline in correlation amounted to 0.07 after five years, indicating that the ability of the FFQ to rank subjects according to nutrient intake maintains relatively well over time. ${ }^{22}$

\section{Statistical analysis}

The Cox proportional hazards model was used to estimate age- and sex-adjusted and multivariable-adjusted incidence rate ratios (RR) and corresponding $95 \%$ confidence intervals $(\mathrm{Cl})$. Person-years at risk were calculated from baseline until diagnosis of HNC, death, emigration, loss to follow-up, or end of follow-up, whichever occurred first. We analyzed total vegetable and fruit consumption, total vegetable consumption, consumption of cooked and raw vegetables, consumption of several vegetable subgroups and the most frequently consumed ( $<30 \%$ non-users in the subcohort) individual vegetables. In addition, we analyzed total fruit consumption, consumption of citrus fruits and the most frequently consumed individual fruits. The composition of each vegetable and fruit group can be found in Table 3.1. For categorical analyses, we categorized vegetable and fruit consumption levels into quartiles (Q) according to the sex-specific distribution in the subcohort; we chose an increment of 25 grams/day for continuous analyses.

The predefined confounders were age (years); sex; alcohol consumption (grams/day); and cigarette smoking (status (never/former/current), number of cigarettes smoked daily, and number of smoking years). Total vegetable and total fruit consumption were mutually adjusted in statistical models. We wanted to investigate whether vegetable and fruit subgroups and/or individual items have a specific additional effect on HNC risk, apart from being vegetables or fruits. Vegetables and fruits share some common characteristics and are, for example, generally low in calories and rich in fiber. However, other potential beneficial components (such as the amount and/or type of vitamins or phytochemicals) may vary among vegetable and fruit subgroups. In order to examine this possible additional effect, analyses of vegetable/fruit subgroups and individual items were adjusted for total vegetable and fruit intake. 
We considered the following potential confounders: level of education; body mass index; non-occupational physical activity; intake of fish, red meat, and meat products (all grams/day); and family history of HNC.,23 A variable was regarded as a confounder if including it in the model changed the RR for intake of total vegetables and fruits (continuous) for HNC overall or any of the HNC subtypes by $>10 \%$. According to this, only the predefined confounders were included in the final model. When adjusting for cigarette smoking frequency and duration, we centered these continuous variables as proposed by Leffondré et al. ${ }^{24}$

We assessed tests for linear dose-response trends by fitting ordinal exposure variables as continuous terms. Standard errors were estimated using the robust HuberWhite sandwich estimator to account for additional variance due to sampling from the cohort; this method is equivalent to Barlow's ${ }^{25}$ variance-covariance estimator. The proportional hazards (PH) assumption was assessed using the scaled Schoenfeld residuals. ${ }^{26}$ If there was an indication for violation of the assumption for a variable, it was further investigated by adding a time-varying covariate for that variable to the model.

We conducted analyses of total vegetable and fruit consumption stratified by sex in order to evaluate possible interaction. No statistically significant interaction with sex was found ( $P$ interaction for HNC overall=0.43); therefore, all analyses were carried out for both sexes combined. To determine whether alcohol consumption and/or cigarette smoking modify the association of total vegetable and fruit intake with HNC risk, we performed analyses in strata of alcohol consumption (abstainers; consuming $>0$ to 15 grams ethanol/day; consuming $\geq 15$ grams ethanol/day) and cigarette smoking status (never/former/current). Alcohol consumption and cigarette smoking were mutually adjusted in these models. $P$ values for interaction were assessed by including crossproduct terms in the models and performing a Wald test.

To prevent bias caused by reversed causation, we checked for possible influence of preclinical cancer at baseline for consumption of vegetables and fruits. First, we compared the median vegetable and fruit consumption of cases diagnosed during the first two years of follow-up with the consumption of cases diagnosed later in follow-up. HNC cases diagnosed after the second year of follow-up $(N=382)$ had a median consumption of 173 grams of vegetables and 109 grams of fruit per day. Cases diagnosed during the first two years of follow-up $(N=33)$ had a daily median consumption of 176 grams of vegetables and 91 grams of fruit.

Second, after we applied a square-root transformation to normalize the distribution of the vegetable and fruit variables, we used an independent samples $t$ test to test the statistical significance of differences; there was no statistically 
significant difference in vegetable $(P=0.65)$ or fruit $(P=0.36)$ consumption between early and late cases. Based on these results, we decided to include the total follow-up time in our analyses. In addition, we performed sensitivity analyses regarding the association between consumption of total vegetables and fruits, total vegetables, and total fruits, and the risk of HNC overall. We found essentially similar results for the total follow-up period and the period with exclusion of the first two years of follow-up (data not shown).

For HNC overall, the PH assumption was violated for current smoking-and a timevarying covariate showed a statistically significant interaction with time-in all categorical and continuous analyses. Although smoking was extensively addressed in the questionnaire (with questions about lifetime exposure history), we lack data after baseline. It is possible that participants who did smoke at baseline stopped smoking at some point during follow-up, which may explain this interaction with time. We did not find this for HNC subtypes, possibly as a result of less power. However, it seems theoretically plausible that the interaction of current smoking with time is also present in OCC, OHPC, and LC. Therefore, we decided to perform analyses for HNC and all HNC subtypes using a time-varying covariate for current smoking.

All reported $P$ values were based on two-sided tests and considered statistically significant if $<0.05$. Analyses were done using the Stata 11.2 statistical software package (StataCorp, College Station, Texas, USA).

\section{Results}

\section{Baseline characteristics}

After 20.3 years of follow-up, 415 cases of HNC overall (131 OCC, 88 OHPC, 3 oral cavity/pharynx unspecified or overlapping, and 193 LC) were available for analysis (Figure 3.1), with a mean follow-up of 16.9 years and a total of 65,980 person-years at risk in the subcohort. Characteristics of our study population are shown in Table 3.2. Daily median total vegetable and fruit consumption was considerably lower among cases (292 grams for HNC overall) than subcohort members (344 grams), almost entirely the result of lower fruit consumption among cases. Compared to the subcohort, cases were more often men than women, especially LC cases. Among subcohort members and cases, women consumed substantially more fruit than men. Furthermore, there were far more current smokers among cases than subcohort members; in addition, cases smoked for a substantially higher number of pack-years. 


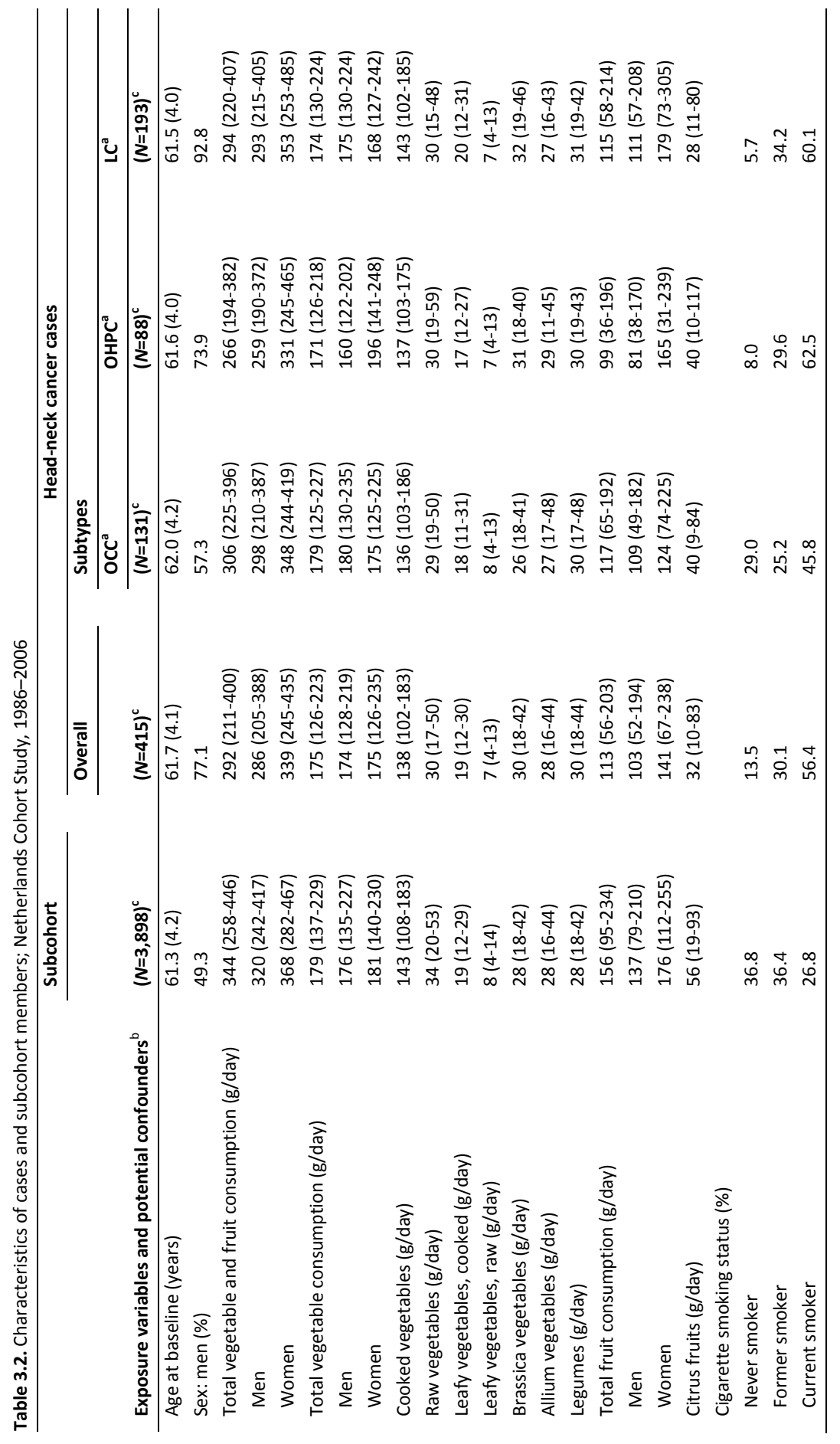




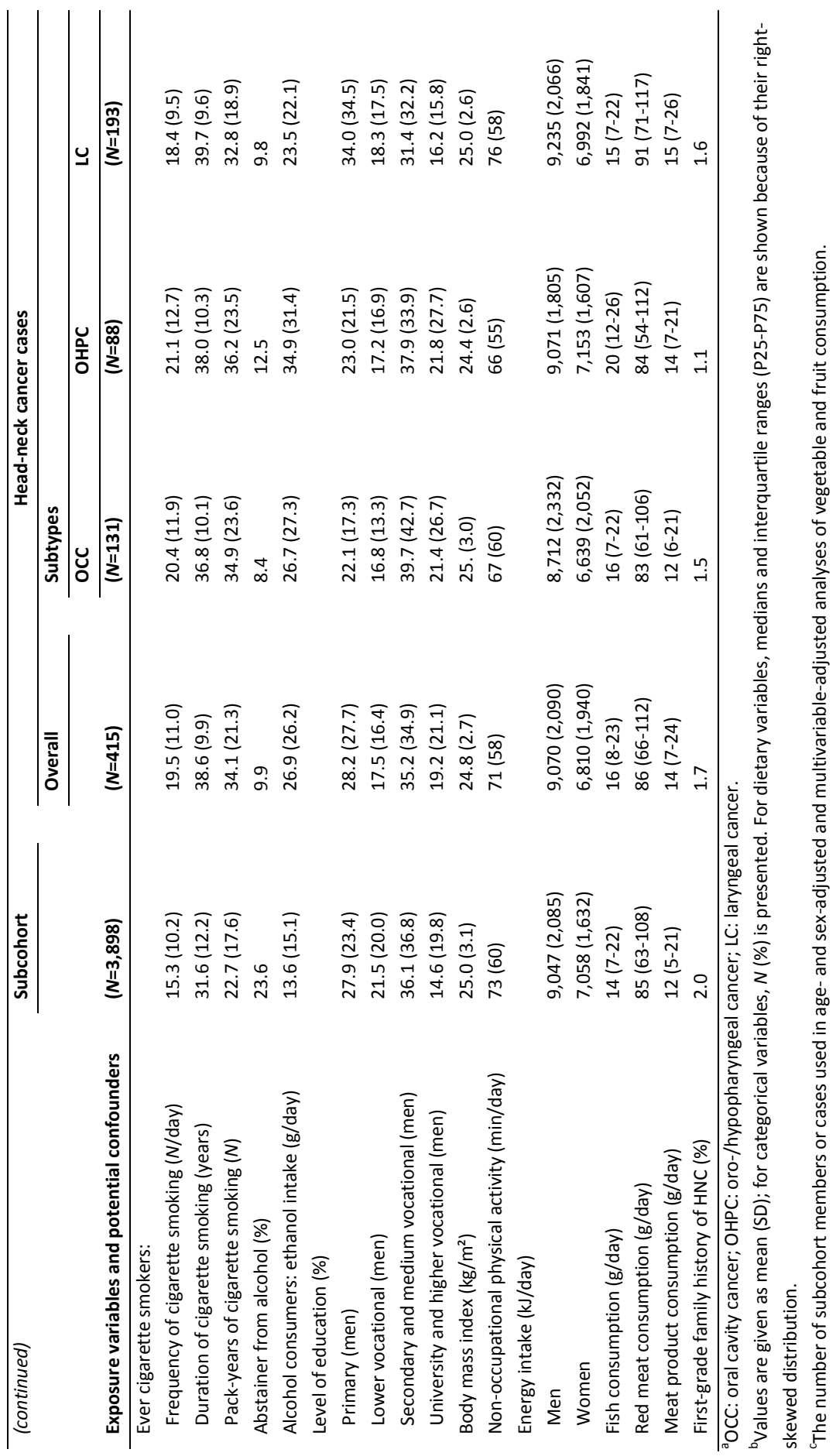


Cases were also less frequently alcohol abstainers and, among alcohol consumers, cases had a considerably higher alcohol intake than subcohort members.

The intake of specific items of vegetables and fruits of HNC overall cases and subcohort members is presented in Supplemental Table 3.1; individual vegetable and fruit items are ranked according to the percentage of non-users in the subcohort. Of the items asked about in the questionnaire, string beans, cauliflower and lettuce were the most frequently eaten vegetables in our study population, while raw carrots, gherkins and vegetable juices were consumed the least. Tomatoes, onions and string beans were the vegetables eaten in the largest amounts. The most consumed fruits in the subcohort were apples and pears, strawberries, and oranges; apples and pears and oranges were also the fruits consumed in the largest amounts.

\section{Main analyses}

Results of the multivariable-adjusted analyses on total vegetable and fruit consumption and vegetable and fruit subgroups are shown in Table 3.3. Results from age- and sex-adjusted analyses (data not shown) were largely comparable to the multivariable-adjusted results, but with most (inverse) associations between vegetable and fruit consumption and HNC risk being stronger in age- and sex-adjusted analyses.

Total vegetable and fruit consumption was associated with a decreased risk of HNC overall and of all HNC subtypes, with statistically significant associations in HNC overall (multivariable-adjusted RR for Q4 vs. Q1: $0.61,95 \% \mathrm{Cl} 0.44-0.85, P$ trend=0.002), OCC (RR: $0.46,95 \% \mathrm{Cl} 0.27-0.81, P$ trend=0.005) and OHPC (RR: 0.51, 95\% Cl 0.26-1.00, $P$ trend=0.03) (Table 3.3). For LC, we found inverse (but not statistically significant) associations as well (RR for Q4 vs. Q1: $0.80,95 \% \mathrm{Cl} 0.51-1.23, P$ trend=0.32). Total vegetable intake was also consistently inversely associated with HNC risk, but only statistically significantly with HNC overall (RR for Q4 vs. Q1: $0.71,95 \% \mathrm{Cl} 0.51-0.99, P$ trend=0.07) (Table 3.3). When investigating the effect of specific groups of vegetables on HNC risk, we found that raw leafy vegetables statistically significantly decreased the risk of HNC overall (RR for Q4 vs. Q1: $0.67,95 \% \mathrm{Cl} 0.47-0.96, P$ trend=0.05), adjusted for total vegetable and fruit intake. Among HNC subtypes, raw leafy vegetables were generally associated with decreased risks as well, but only with statistical significance in OHPC (RR for Q4 vs. Q1: 0.46, 95\% Cl 0.23-0.93, $P$ trend=0.11). Point estimates (Q4 vs. Q1 or per $25 \mathrm{~g} /$ day increment) for other vegetable subgroups varied below and above unity, and were not statistically significant (neither for HNC overall nor for HNC subtypes). 


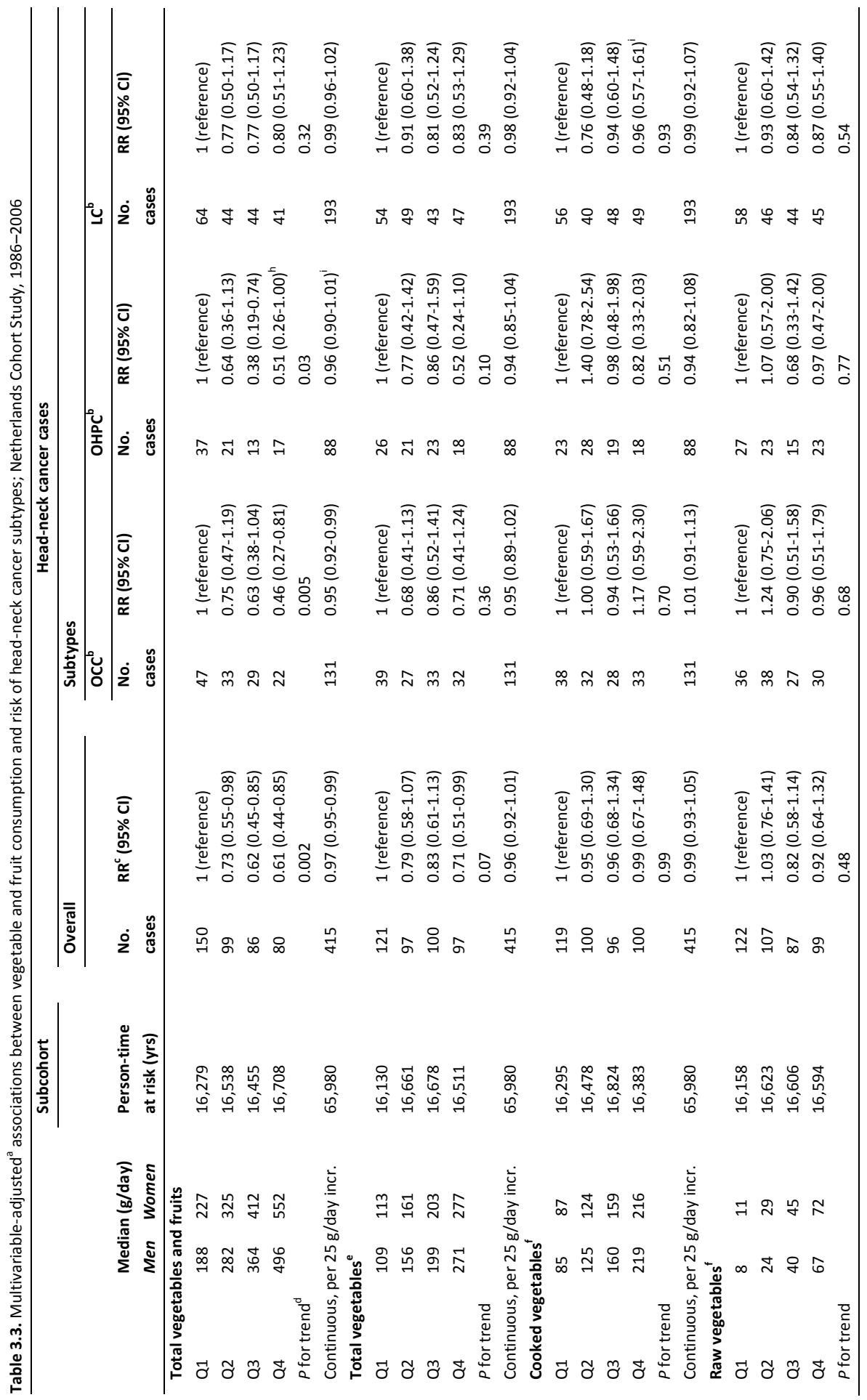




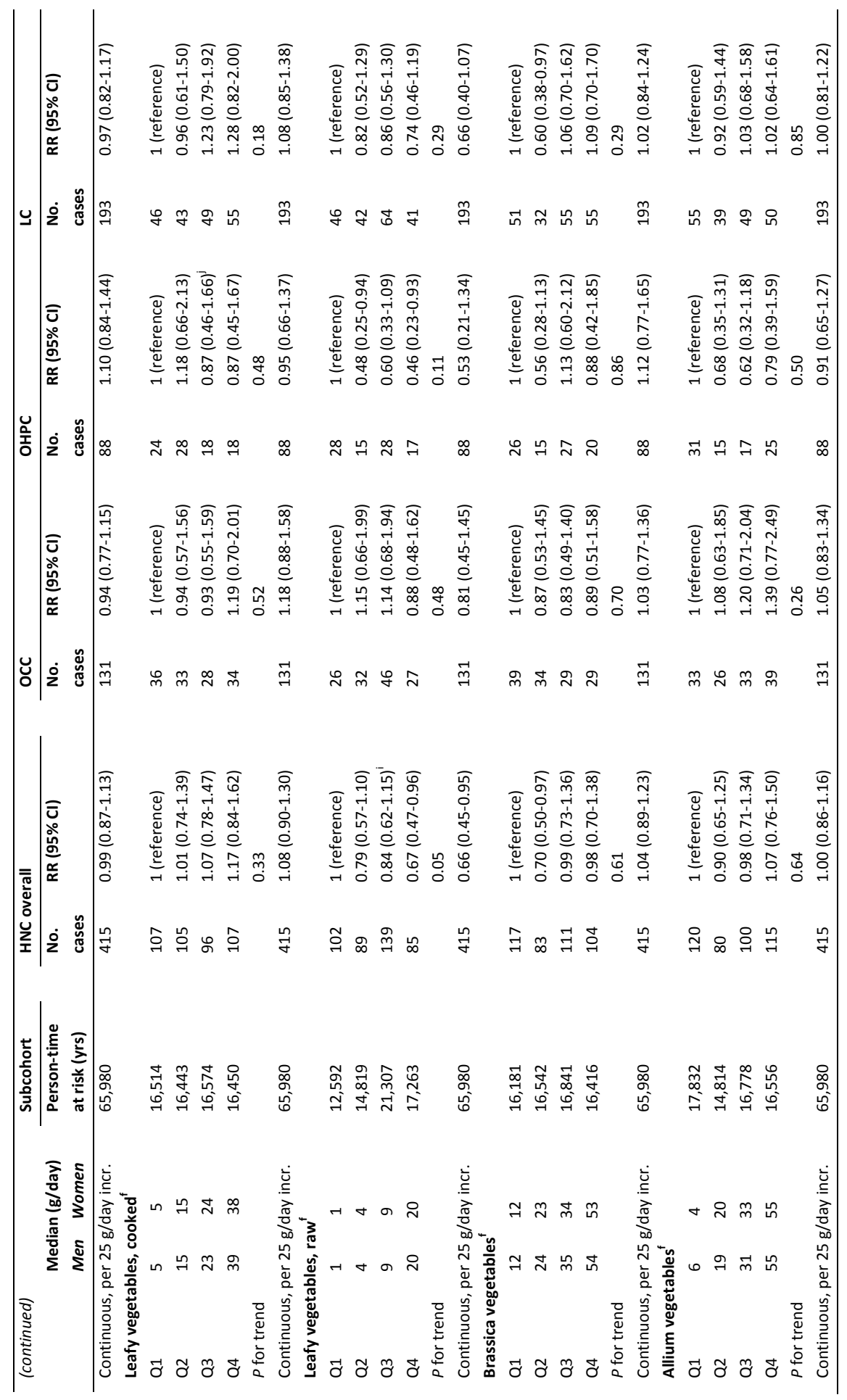




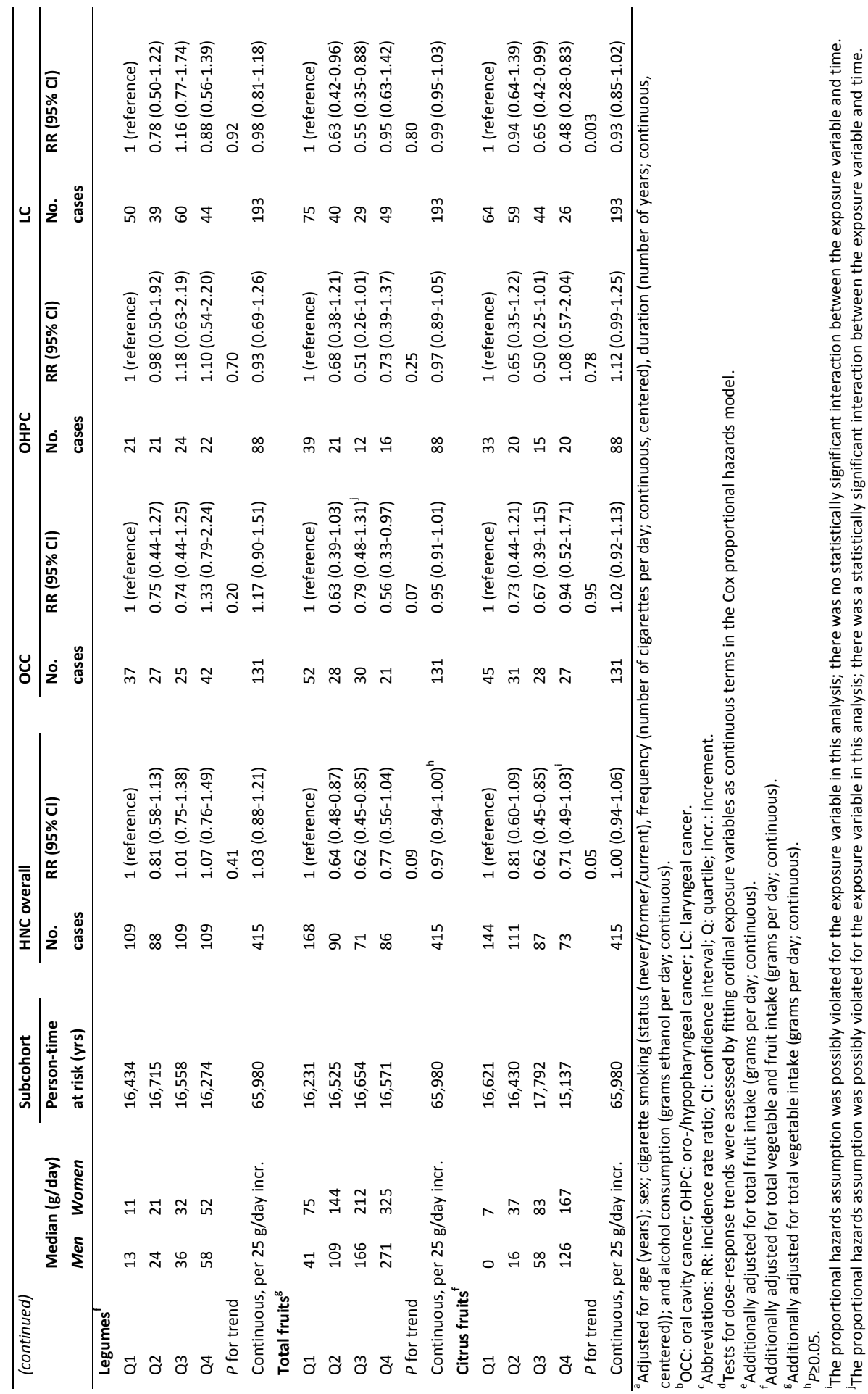




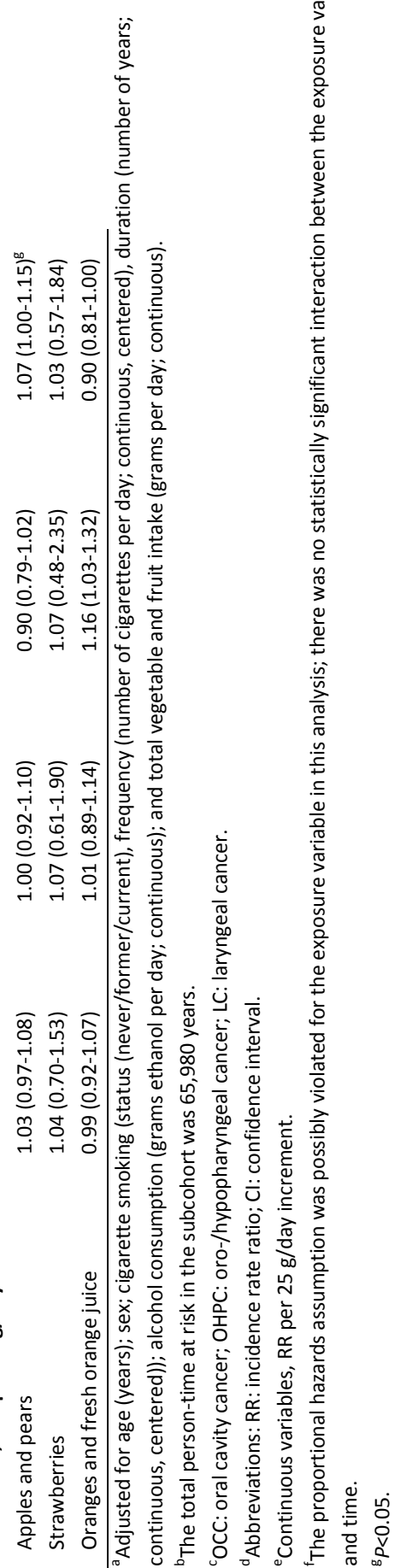


Total fruit consumption was also associated with decreased risks of HNC overall and of all subtypes. All point estimates were below unity, but no clear trend was observed and statistically significant associations were only found when comparing Q3 vs. Q1 and Q2 vs. Q1 in HNC overall and in LC, and Q4 vs. Q1 in OCC (Table 3.3). Citrus fruits were clearly statistically significantly associated with a decreased risk of LC (RR for Q4 vs. Q1: $0.48,95 \% \mathrm{Cl} 0.28-0.83, P$ trend=0.003).

With respect to individual vegetable and fruit items (Table 3.4), only lettuce wasafter adjustment for total vegetable and fruit consumption-statistically significantly associated with a decreased HNC risk, namely for HNC overall (RR per 25 grams/day: $0.58,95 \% \mathrm{Cl} 0.35-0.95)$. After adjustment for total vegetable and fruit intake, we found positive associations for consumption of oranges and fresh orange juice and OHPC (RR per 25 grams/day: 1.16, 95\% Cl 1.03-1.32), spinach and LC (RR per 25 grams/day: 1.54, $95 \% \mathrm{Cl}$ 1.03-2.29), and apples and pears and LC (RR per 25 grams/day: $1.07,95 \% \mathrm{Cl}$ $1.00-1.15)$.

\section{Stratified analyses}

Results of the multivariable-adjusted stratified analyses are presented in Table 3.5. Among current smokers, we found statistically significantly decreased risks of HNC overall for participants with the highest vegetable and fruit consumption (those in Q4 (RR: $0.52,95 \% \mathrm{Cl} 0.31-0.88$ ) and Q3 (RR: $0.54,95 \% \mathrm{Cl} 0.34-0.86)$ ) compared to current smokers in Q1 as a reference group. No statistically significant interaction was found between categories of cigarette smoking status and vegetable and fruit intake (Q1-Q4) for HNC overall ( $P$ interaction=0.10).

We found a similar pattern for alcohol consumption, with statistically significantly decreased risks of HNC overall among participants who drank $\geq 15$ grams/day and had the highest vegetable and fruit consumption (those in Q4 (RR: $0.48,95 \% \mathrm{Cl} 0.30-0.77$ ) and Q3 (RR: $0.47,95 \% \mathrm{Cl} 0.30-0.74)$ ). No statistically significant interaction was found between alcohol consumption and vegetable and fruit intake (Q1-Q4) for HNC overall ( $P$ interaction=0.09). 
Table 3.5. Stratified analyses of sex/cigarette smoking/alcohol consumption and quartiles of vegetable and fruit consumption and risk (multivariable-adjusted ${ }^{\mathrm{a}}$ associations) of head-neck cancer overall; Netherlands Cohort Study, 1986-2006

\begin{tabular}{|c|c|c|c|c|}
\hline \multicolumn{5}{|c|}{ Head-neck cancer overall } \\
\hline & \multicolumn{4}{|c|}{ Vegetable and fruit consumption } \\
\hline & $Q 1^{b}$ & Q2 & Q3 & Q4 \\
\hline \multicolumn{5}{|l|}{ Sex } \\
\hline \multicolumn{5}{|l|}{ M } \\
\hline Cases/person-time at risk (years) & $110 / 7,493$ & $82 / 7,752$ & $65 / 7,553$ & $63 / 7,871$ \\
\hline $\mathrm{RR}(95 \% \mathrm{Cl})$ & 1 (ref) & $0.82(0.58-1.15)$ & $0.63(0.43-0.90)$ & $0.63(0.43-0.93)$ \\
\hline \multicolumn{5}{|l|}{$\mathrm{F}$} \\
\hline Cases/person-time at risk (years) & $40 / 8,786$ & $17 / 8,786$ & $21 / 8,902$ & $17 / 8,837$ \\
\hline $\mathrm{RR}(95 \% \mathrm{Cl})$ & 1 (ref) & $0.48(0.26-0.89)$ & $0.59(0.33-1.06)$ & $0.48(0.25-0.92)$ \\
\hline$P$ for interaction ${ }^{c}$ & 0.41 & & & \\
\hline \multicolumn{5}{|l|}{ Cigarette smoking } \\
\hline \multicolumn{5}{|l|}{ Never } \\
\hline Cases/person-time at risk (years) & $14 / 5,948$ & $13 / 6,196$ & $17 / 6,596$ & $12 / 7,095$ \\
\hline $\mathrm{RR}(95 \% \mathrm{Cl})$ & 1 (ref) & $0.84(0.38-1.85)$ & $1.20(0.57-2.53)$ & $0.54(0.22-1.29)$ \\
\hline \multicolumn{5}{|l|}{ Former } \\
\hline Cases/person-time at risk (years) & $39 / 4,919$ & $22 / 6,179$ & $27 / 6,037$ & $37 / 6,588$ \\
\hline $\mathrm{RR}(95 \% \mathrm{Cl})$ & 1 (ref) & $0.45(0.26-0.79)$ & $0.59(0.34-1.00)$ & $0.72(0.44-1.18)$ \\
\hline \multicolumn{5}{|l|}{ Current } \\
\hline Cases/person-time at risk (years) & $97 / 5,413$ & $64 / 4,163$ & $42 / 3,822$ & $31 / 3,026$ \\
\hline $\mathrm{RR}(95 \% \mathrm{Cl})$ & 1 (ref) & $0.89(0.60-1.31)$ & $0.54(0.34-0.86)$ & $0.52(0.31-0.88)$ \\
\hline$P$ for interaction & 0.10 & & & \\
\hline \multicolumn{5}{|l|}{ Alcohol consumption } \\
\hline \multicolumn{5}{|l|}{ Abstainers } \\
\hline Cases/person-time at risk (years) & $14 / 4,228$ & $12 / 3,784$ & $5 / 3,676$ & $10 / 3,720$ \\
\hline $\mathrm{RR}(95 \% \mathrm{Cl})$ & 1 (ref) & $1.06(0.47-2.35)$ & $0.47(0.17-1.36)$ & $0.89(0.35-2.24)$ \\
\hline \multicolumn{5}{|l|}{$>0-15$ (g/day) } \\
\hline Cases/person-time at risk (years) & $50 / 8,340$ & $34 / 8,819$ & $41 / 8,553$ & $34 / 8,700$ \\
\hline $\mathrm{RR}(95 \% \mathrm{Cl})$ & 1 (ref) & $0.69(0.43-1.11)$ & $0.97(0.62-1.53)$ & $0.79(0.49-1.28)$ \\
\hline \multicolumn{5}{|l|}{$\geq 15$ (g/day) } \\
\hline Cases/person-time at risk (years) & $86 / 3,711$ & $53 / 3,935$ & $40 / 4,225$ & $36 / 4,287$ \\
\hline $\mathrm{RR}(95 \% \mathrm{Cl})$ & 1 (ref) & $0.71(0.46-1.09)$ & $0.47(0.30-0.74)$ & $0.48(0.30-0.77)$ \\
\hline$P$ for interaction & 0.09 & & & \\
\hline
\end{tabular}

${ }^{a}$ Mutually adjusted for age (years); sex; cigarette smoking (status (never/former/current), frequency (number of cigarettes per day; continuous, centered), duration (number of years; continuous, centered)); and alcohol consumption (grams ethanol per day; continuous).

${ }^{\mathrm{b}}$ Abbreviations: RR: incidence rate ratio; $\mathrm{Cl}$ : confidence interval; Q: quartile.

${ }^{\mathrm{C}} P$ value for interaction based on cross-product terms in the Cox proportional hazards model and Wald test. 


\section{Discussion}

In this large prospective cohort study, we found significant inverse associations between consumption of vegetables and fruits and risk of HNC. Total vegetable and fruit consumption was associated with a substantially decreased risk of $0.61(95 \% \mathrm{Cl}$ 0.44-0.85; Q4 vs. Q1) for HNC overall; among subtypes, OCC was most strongly inversely associated with total vegetable and fruit intake. Total vegetable consumption was inversely associated with risk of HNC overall as well; associations with HNC subtypes did not reach statistical significance and were largely similar for OCC, OHPC, and LC. Total fruit consumption was associated with reduced risks-but not a consistent trend-of HNC overall and all subtypes; the strongest associations were with OCC. Associations between vegetable and fruit consumption and HNC risk were not clearly modified by cigarette smoking status or alcohol consumption.

\section{Total vegetables and fruits, subgroups, and individual items}

The results of our study are generally in line with those of previous studies (summarized in a systematic literature review by the World Cancer Research Fund ${ }^{3}$ ) which showed consistent inverse associations between intake of total vegetables and fruits and HNC risk. ${ }^{5,7,9,10,27-29}$ However, the strength of these associations varied, possibly as a result of differences in study design, case definition and/or exposure assessment. In the NIH-AARP Diet and Health cohort, a hazard ratio (HR) of 0.71 (95\% $\mathrm{Cl}$ 0.55-0.92) for total fruit and vegetable intake was reported, comparing Q5 vs. Q1. ${ }^{9}$ In the EPIC study, an estimated relative risk of 0.60 (95\% Cl 0.37-0.99; Q5 vs. Q1) was observed for consumption of total fruits and vegetables and risk of UADTC, which also includes esophageal cancer. ${ }^{7}$ Furthermore, in the NIH-AARP cohort it was found that HNC risk might be more strongly inversely associated with vegetables than with fruits, which appears to be the same in our study. However, the EPIC study found that fruits had a stronger effect.

Regarding the difference we found among HNC subtypes, consumption of total fruit and vegetables, and of vegetables alone, was also most strongly associated with OCC in the NIH-AARP cohort ${ }^{9}$; other prospective studies did not examine OCC, OHPC, and LC separately. A meta-analysis of case-control studies showed that consumption of fruit and vegetables had stronger protective effects for mouth and pharynx cancer than for LC. ${ }^{28}$

Prospective data regarding vegetable and fruit subgroups and individual items and HNC risk shows inconsistent associations and differences from our results as well. ${ }^{3,7-11}$ This may be because other studies handled different subgroup classifications, studied 
other food items or adjusted for confounders in their analyses differently. We found that raw leafy vegetables and citrus fruits, when adjusted for total vegetable and fruit intake, may exert an additional effect on the risk of HNC overall and LC, respectively. The NIH-AARP cohort analysis ${ }^{9}$ used botanical subgroup classifications and found significantly lower risks for HNC overall for leguminosae (beans, peas), rosaceae (apples, peaches, nectarines, strawberries), solanacea (peppers/tomatoes) and umbelliferae (carrots), but not for chenopodiaceae (raw spinach and cooked spinach), compositae (lettuce) and rutaceae (citrus fruit). In the prospective EPIC study ${ }^{7}$, vegetable subgroups were not related to risk of UADTC (with the exception of intake of root vegetables in men). Raw (leafy) vegetables were not examined as a separate vegetable subgroup and citrus fruits were not statistically significantly associated with decreased UADTC-risk. ${ }^{7}$ A prospective study of Norwegian men ${ }^{11}$ found that oranges were significantly inversely related to upper aerogastric tract cancer, but the study only included 71 cases ( 28 oral and pharyngeal, 21 laryngeal, and 22 esophageal cancer cases). It found no effect for highest vs. lowest intake of lettuce. In conclusion, certain vegetable and fruit subgroups and items may offer stronger HNC risk reduction than others, but the evidence from prospective data remains inconclusive. Evidence from case-control studies regarding vegetable and fruit subgroups shows that raw vegetables and citrus fruits are consistently associated with reduced HNC risk estimates. ${ }^{3}$

\section{Possible interaction between alcohol, smoking, and total vegetable and fruit intake}

We found no significant differences in associations for vegetable and fruit intake and HNC overall among strata of cigarette smoking and alcohol consumption. Similar to our results, the NIH-AARP Diet and Health cohort and the EPIC study found no statistically significant differences for total vegetable and fruit intake and HNC risk by smoking status or alcohol consumption. ${ }^{7,9}$ However, our study found that both current smokers and participants who drink $\geq 15$ grams of alcohol per day while eating the highest amount of vegetables and fruits had the lowest risk of HNC overall. Our non-significant findings regarding the interaction might be due to limited power to detect a significant deviation from the multiplicative model.

\section{Mechanisms of action}

Several mechanisms of action that vegetables and fruits have on HNC risk have been described, mainly the potential anticarcinogenic properties of numerous components present in vegetables and fruits. ${ }^{3,5}$ These components include vitamins, fiber, folate, flavonoids and carotenoids, some of which are potential antioxidants and might play a 
role in several processes, such as protection against oxidative stress and DNA repair. These substances may have an indirect, systemic effect (i.e., they become available in the body after being metabolized and subsequently act in numerous processes across the body), but they may also exert their effect on HNC carcinogenesis through direct exposure to tissue in the head-neck area. This is a possible explanation for the differential risk among HNC subtypes, as the larynx is being minimally exposed to foods and drinks compared with the oral cavity and pharynx.

There might be other-less described, yet potentially interesting-mechanisms for the effect of vegetables and fruits on HNC risk. One potential mechanism regarding the association between vegetables, fruits and HNC risk might be the beneficial effect that vegetables-especially raw vegetables-and fruits have on the body's acid-base balance by promoting alkalinity, thereby possibly influencing tumor growth. ${ }^{30,31}$ Furthermore, a diet high in vegetables and fruits may influence the process of lowgrade, chronic inflammation in the body, which might be involved in cancer risk as well. $^{32-34}$ Given that perspective, it might also be interesting to examine dietary patterns and HNC risk, since people who eat more vegetables and fruits might have a generally healthier lifestyle, reflecting another dietary pattern than that of people who consume more animal and/or processed foods. ${ }^{35,36}$ So far, a dietary pattern reflecting relatively high fruit/vegetable intake and low intake of animal/processed foods has been associated with reduced $\mathrm{HNC}$ risk. ${ }^{35-37}$

\section{Strengths and limitations}

Important strengths of our study are the prospective nature and completeness and duration of follow-up. Given our large number of cases, we were able to examine the effect of vegetable and fruit consumption not only on overall HNC risk but also on the risk of HNC subtypes. Furthermore, we were able to thoroughly adjust for confounding by the main HNC risk factors (smoking and alcohol consumption), including information on smoking duration.

A possible limitation of our study is the single measurement of exposure data by our questionnaire. Although vegetable and fruit intake was extensively addressed, the FFQ may have provided only moderately accurate estimates of vegetable and fruit consumption. In addition, we believe the FFQ was given to people in an age group that had stable dietary habits at baseline, but it is nevertheless possible that participants changed their dietary habits since 1986. As a consequence, bias due to random misclassification may have occurred, possibly resulting in an underestimation of the effect of vegetables and fruits on HNC risk. The true risk lowering effect of vegetable and fruit consumption on HNC may therefore be even stronger than we found. 
Furthermore, although we have detailed information about alcohol consumption and cigarette smoking in our cohort and were therefore able to adjust for these confounders extensively, we cannot exclude the presence of some residual confounding by smoking and/or alcohol. ${ }^{27}$ Age- and sex-adjusted results were, however, largely comparable with multivariable-adjusted results. Residual confounding may also have occurred due to other potential confounders, but as we investigated many of these, we presume this to be limited as well. Finally, for reasons of efficiency, we used listwise deletion to handle missing data on confounders. This statistical approach resulted in only a minor loss of power when compared to the use of multiple imputation, but might have led to bias as a result of removing data with missing values. $^{38}$

Our study lacks information on HPV infection, which has been associated with HNC risk. $^{4,39-41}$ Bias due to potential misclassification with regard to HPV is therefore possible, which makes the lack of information on HPV a limitation of our study. Other factors we did not take into account are the use of drugs and oral hygiene.

Finally, though we wanted to examine the role of vegetable and fruit consumption in HNC subtypes, we did not investigate HNC located in the major salivary glands, nasal cavity, paranasal sinuses and nasopharynx because of a presumably different etiology as well as low case numbers.

\section{Conclusions}

The present study, the second largest prospective study regarding this topic so far, confirms and further establishes that vegetable and fruit consumption is associated with reduced risk of developing HNC and HNC subtypes. Specific groups of vegetables and fruits may have an additional HNC risk reducing effect. In future research, it might be interesting to further elucidate certain mechanisms which may or may not be involved in (head-neck) cancer risk and development.

\section{References}

1 Argiris, A., Karamouzis, M. V., Raben, D. \& Ferris, R. L. Head and neck cancer. Lancet 371, 1695-1709 (2008).

2 Ferlay J. et al. GLOBOCAN 2008 v1.2, Cancer Incidence and Mortality Worldwide: IARC CancerBase No. 10 [Internet]. Lyon, France: International Agency for Research on Cancer; 2010. Available from: http://globocan.iarc.fr, accessed on 30/05/2013.

3 World Cancer Research Fund \& American Institute for Cancer Research. Food, Nutrition, Physical Activity and the Prevention of Cancer: a Global Perspective. American Institute for Cancer Research, 2007.

4 IARC. A Review of Human Carcinogens: Biological Agents. Vol. 100B International Agency for Research on Cancer, 2012. 
5 International Agency for Research on Cancer \& World Health Organization. IARC Handbooks of Cancer Prevention: Fruit and Vegetables. IARC Press, 2003.

6 Dallongeville, J., Marecaux, N., Fruchart, J. C. \& Amouyel, P. Cigarette smoking is associated with unhealthy patterns of nutrient intake: a meta-analysis. J Nutr 128, 1450-1457 (1998).

7 Boeing, H. et al. Intake of fruits and vegetables and risk of cancer of the upper aero-digestive tract: the prospective EPIC-study. Cancer Causes Control 17, 957-969 (2006).

8 Chyou, P. H., Nomura, A. M. \& Stemmermann, G. N. Diet, alcohol, smoking and cancer of the upper aerodigestive tract: a prospective study among Hawaii Japanese men. Int J Cancer 60, 616-621 (1995).

9 Freedman, N. D. et al. Fruit and vegetable intake and head and neck cancer risk in a large United States prospective cohort study. Int J Cancer 122, 2330-2336 (2008).

10 Kasum, C. M., Jacobs, D. R., Jr., Nicodemus, K. \& Folsom, A. R. Dietary risk factors for upper aerodigestive tract cancers. Int J Cancer 99, 267-272 (2002).

11 Kjaerheim, K., Gaard, M. \& Andersen, A. The role of alcohol, tobacco, and dietary factors in upper aerogastric tract cancers: a prospective study of 10,900 Norwegian men. Cancer Causes Control 9, 99-108 (1998).

12 Freedman, N. D., Abnet, C. C., Leitzmann, M. F., Hollenbeck, A. R. \& Schatzkin, A. Prospective investigation of the cigarette smoking-head and neck cancer association by sex. Cancer 110, 1593-1601 (2007).

13 Dobrossy, L. Epidemiology of head and neck cancer: magnitude of the problem. Cancer Metastasis Rev 24, 9-17 (2005).

14 van den Brandt, P. A. et al. A large-scale prospective cohort study on diet and cancer in The Netherlands. J Clin Epidemiol 43, 285-295 (1990).

15 Barlow, W. E., Ichikawa, L., Rosner, D. \& Izumi, S. Analysis of case-cohort designs. J Clin Epidemiol 52, 1165-1172 (1999).

16 van den Brandt, P. A., Schouten, L. J., Goldbohm, R. A., Dorant, E. \& Hunen, P. M. Development of a record linkage protocol for use in the Dutch Cancer Registry for Epidemiological Research. Int J Epidemiol 19, 553-558 (1990).

17 Goldbohm RA, Van den Brandt PA \& Dorant E. Estimation of the coverage of Dutch municipalities by cancer registries and PALGA based on hospital discharge data. Tijdschr Soc Gezondheidsz, 80-84 (1994).

18 Goldbohm, R. A. et al. Validation of a dietary questionnaire used in a large-scale prospective cohort study on diet and cancer. Eur J Clin Nutr 48, 253-265 (1994).

19 Heinen, M. M., Verhage, B. A., Goldbohm, R. A. \& van den Brandt, P. A. Intake of vegetables, fruits, carotenoids and vitamins $C$ and $E$ and pancreatic cancer risk in The Netherlands Cohort Study. Int J Cancer 130, 147-158 (2012).

20 Hashibe, M. et al. Alcohol drinking in never users of tobacco, cigarette smoking in never drinkers, and the risk of head and neck cancer: pooled analysis in the International Head and Neck Cancer Epidemiology Consortium. J Nat/ Cancer Inst 99, 777-789 (2007).

21 Fritz, A. G. International Classification of Diseases for Oncology: ICD-O. 3rd edn, World Health Organization, 2000.

22 Goldbohm, R. A. et al. Reproducibility of a food frequency questionnaire and stability of dietary habits determined from five annually repeated measurements. Eur J Clin Nutr 49, 420-429 (1995).

23 Negri, E. et al. Family history of cancer: pooled analysis in the International Head and Neck Cancer Epidemiology Consortium. Int J Cancer 124, 394-401 (2009).

24 Leffondre, K., Abrahamowicz, M., Siemiatycki, J. \& Rachet, B. Modeling smoking history: a comparison of different approaches. Am J Epidemiol 156, 813-823 (2002).

25 Barlow, W. E. Robust variance estimation for the case-cohort design. Biometrics 50, 1064-1072 (1994).

26 Schoenfeld, D. Partial residuals for the proportional hazards regression model. Biometrika 69, 239-241 (1982).

27 Key, T. J. Fruit and vegetables and cancer risk. Br J Cancer 104, 6-11 (2011).

28 Riboli, E. \& Norat, T. Epidemiologic evidence of the protective effect of fruit and vegetables on cancer risk. Am J Clin Nutr 78, 559S-569S (2003).

29 Pavia, M., Pileggi, C., Nobile, C. G. \& Angelillo, I. F. Association between fruit and vegetable consumption and oral cancer: a meta-analysis of observational studies. Am J Clin Nutr 83, 1126-1134 (2006).

30 Robey, I. F. Examining the relationship between diet-induced acidosis and cancer. Nutr Metab (Lond) 9, 72 (2012).

31 Welch, A. A., Mulligan, A., Bingham, S. A. \& Khaw, K. T. Urine $\mathrm{pH}$ is an indicator of dietary acid-base load, fruit and vegetables and meat intakes: results from the European Prospective Investigation into Cancer and Nutrition (EPIC)Norfolk population study. Br J Nutr 99, 1335-1343 (2008).

32 Cao, Y., DePinho, R. A., Ernst, M. \& Vousden, K. Cancer research: past, present and future. Nat Rev Cancer 11, 749-754 (2011).

33 Coussens, L. M. \& Werb, Z. Inflammation and cancer. Nature 420, 860-867 (2002). 
34 Julia, C. et al. Dietary patterns and risk of elevated C-reactive protein concentrations 12 years later. Br J Nutr 110, 747754 (2013).

35 Bradshaw, P. T. et al. Associations between dietary patterns and head and neck cancer: the Carolina head and neck cancer epidemiology study. Am J Epidemiol 175, 1225-1233 (2012).

36 Bravi, F. et al. Dietary patterns and upper aerodigestive tract cancers: an overview and review. Ann Oncol 23, 30243039 (2012).

37 Chuang, S. C. et al. Diet and the risk of head and neck cancer: a pooled analysis in the INHANCE consortium. Cancer Causes Control 23, 69-88 (2012).

38 Greenland, S. \& Finkle, W. D. A critical look at methods for handling missing covariates in epidemiologic regression analyses. Am J Epidemiol 142, 1255-1264 (1995).

39 Hobbs, C. G. et al. Human papillomavirus and head and neck cancer: a systematic review and meta-analysis. Clin Otolaryngol 31, 259-266 (2006).

40 Marur, S., D'Souza, G., Westra, W. H. \& Forastiere, A. A. HPV-associated head and neck cancer: a virus-related cancer epidemic. Lancet Oncol 11, 781-789 (2010).

41 Mehanna, H. et al. Prevalence of human papillomavirus in oropharyngeal and nonoropharyngeal head and neck cancer--systematic review and meta-analysis of trends by time and region. Head Neck 35, 747-755 (2013). 
Supplemental Table 3.1. Daily median intake and interquartile ranges of specific items of vegetables and fruits of cases and subcohort members; Netherlands Cohort Study, 1986-2006

\begin{tabular}{|c|c|c|c|c|c|c|}
\hline \multirow[b]{3}{*}{ Food group or food item ${ }^{c}$} & \multicolumn{3}{|c|}{ Subcohort $(N=3,898)^{a}$} & \multicolumn{3}{|c|}{$\mathrm{HNC}^{\mathrm{b}}$ overall $(\mathrm{N}=415)^{\mathrm{a}}$} \\
\hline & \multirow[t]{2}{*}{$\begin{array}{c}\text { Nonusers } \\
\text { (\%) }\end{array}$} & \multicolumn{2}{|c|}{$\begin{array}{l}\text { Users: daily } \\
\text { intake (g) }\end{array}$} & \multirow[t]{2}{*}{$\begin{array}{c}\text { Nonusers } \\
\text { (\%) }\end{array}$} & \multicolumn{2}{|c|}{$\begin{array}{l}\text { Users: daily } \\
\text { intake (g) }\end{array}$} \\
\hline & & Median & P25-P75 & & Median & P25-P75 \\
\hline Total vegetables & 0 & 179 & $137-229$ & 0 & 175 & $126-223$ \\
\hline String/French beans & 2 & 17 & $10-25$ & 3 & 17 & $10-25$ \\
\hline Cauliflower & 7 & 13 & $8-20$ & 12 & 14 & $9-20$ \\
\hline Lettuce & 9 & 7 & $4-11$ & 14 & 7 & $4-9$ \\
\hline Carrots, cooked & 12 & 8 & $4-13$ & 17 & 8 & $4-12$ \\
\hline Endive, cooked & 14 & 12 & $7-18$ & 14 & 10 & $6-20$ \\
\hline Brussels sprouts & 15 & 8 & $5-12$ & 15 & 8 & $5-13$ \\
\hline Sauerkraut & 16 & 6 & $3-9$ & 14 & 5 & $3-9$ \\
\hline Tomatoes & 18 & 24 & $14-33$ & 21 & 24 & $14-35$ \\
\hline Onion & 20 & 22 & $11-33$ & 22 & 22 & $11-33$ \\
\hline Spinach & 20 & 10 & $6-16$ & 17 & 11 & $6-17$ \\
\hline Beetroot & 25 & 9 & $5-14$ & 30 & 8 & $5-13$ \\
\hline Kale & 25 & 4 & $2-5$ & 24 & 4 & $2-6$ \\
\hline Cabbage & 29 & 8 & $4-13$ & 30 & 8 & $4-13$ \\
\hline Leek & 32 & 10 & $5-17$ & 32 & 11 & $6-17$ \\
\hline Dried pulses ${ }^{d}$ & 39 & 11 & $4-17$ & 38 & 11 & $6-19$ \\
\hline Mushrooms & 39 & 4 & $4-9$ & 40 & 4 & $4-9$ \\
\hline Broad beans & 43 & 6 & $3-10$ & 35 & 6 & $4-11$ \\
\hline Sweet peppers & 47 & 4 & $3-6$ & 52 & 4 & $3-5$ \\
\hline Endive, raw & 56 & 4 & $2-7$ & 59 & 4 & $2-6$ \\
\hline Rhubarb & 59 & 3 & $1-6$ & 65 & 3 & $1-6$ \\
\hline Carrots, raw & 68 & 5 & $2-10$ & 77 & 4 & $1-9$ \\
\hline Gherkins & 70 & 3 & $1-6$ & 67 & 2 & $1-5$ \\
\hline Vegetable juices $^{d}$ & 90 & 12 & $5-23$ & 90 & 12 & $5-23$ \\
\hline Total fruits & 1 & 156 & $95-234$ & 3 & 113 & $56-203$ \\
\hline Apples and pears & 13 & 80 & $45-116$ & 21 & 45 & $18-116$ \\
\hline Strawberries & 14 & 7 & $4-11$ & 16 & 5 & $2-9$ \\
\hline $\begin{array}{l}\text { Oranges and fresh } \\
\text { orange juice }\end{array}$ & 16 & 39 & $15-83$ & 27 & 26 & $9-83$ \\
\hline Grapes & 37 & 3 & $1-7$ & 44 & 3 & $1-9$ \\
\hline Mandarins & 41 & 4 & $2-8$ & 49 & 3 & $2-8$ \\
\hline Bananas & 47 & 11 & $4-19$ & 54 & 11 & $4-19$ \\
\hline Processed fruit juices ${ }^{d}$ & 50 & 23 & $12-61$ & 62 & 23 & $12-60$ \\
\hline $\begin{array}{l}\text { Grapefruits and fresh } \\
\text { grapefruit juices }\end{array}$ & 71 & 16 & $5-40$ & 77 & 16 & $4-40$ \\
\hline $\begin{array}{l}\text { Raisins and other dried } \\
\text { fruits }\end{array}$ & 75 & 1 & $1-3$ & 86 & 1 & $1-2$ \\
\hline
\end{tabular}

${ }^{\mathrm{a}}$ The number of subcohort members and head-neck cancer cases used in age- and sex-adjusted and multivariableadjusted analyses of vegetable and fruit consumption.

${ }^{\mathrm{b}} \mathrm{HNC}$ : head-neck cancer.

${ }^{c}$ Values are given as medians and interquartile ranges because of the right-skewed distribution of vegetable and fruit items.

${ }^{\mathrm{d} D r i e d}$ pulses and vegetable juices are not included in total vegetable consumption. Processed fruit juices are not included in total fruit consumption. 
Chapter 4

\title{
Vitamin and carotenoid intake and the risk of head-neck cancer subtypes in the Netherlands Cohort Study
}

\author{
Leonie de Munter \\ Denise H. E. Maasland \\ Piet $A$. van den Brandt \\ Bernd Kremer \\ Leo J. Schouten
}




\begin{abstract}
Background

Head-neck cancer (HNC) is the seventh most common type of cancer worldwide. Evidence regarding the potential protective effect of vitamins and carotenoids on HNC is limited and mostly based on case-control studies. We evaluated the association of the intake of dietary vitamins $C$ and $E$ (including supplementation), and the most common carotenoids (alpha-carotene, beta-carotene, lutein/zeaxanthin, lycopene, and beta-cryptoxanthin) on the risk of HNC (subtypes) within the Netherlands Cohort Study.
\end{abstract}

\title{
Methods
}

At baseline in 1986, 120,852 participants completed a food frequency questionnaire. For efficiency reasons, a case-cohort design was used. A subcohort was randomly selected from the total cohort. After 20.3 years of follow-up, 3,898 subcohort members and 415 HNC cases (131 oral cavity cancer (OCC), 88 oro-/hypopharyngeal cancer (OHPC), and 193 laryngeal cancer (LC)) were available for analysis. Incidence rate ratios (RRs) and 95\% confidence intervals (Cl) for highest (Q4) vs. lowest quartile (Q1) of vitamin and carotenoid intake were estimated using the Cox proportional hazards model.

\section{Results}

A strong inverse association was found between vitamin $\mathrm{C}$ and $\mathrm{HNC}$ overall (multivariable-adjusted RR for Q4 vs. Q1: $0.39,95 \% \mathrm{Cl} 0.23-0.66, P$ trend $<0.001$ ), OCC (0.35, 95\% Cl 0.16-0.77, $P$ trend<0.05) and OHPC $(0.29,95 \% \mathrm{Cl} 0.12-0.67, P$ trend<0.01). No statistically significant results were found for vitamin $E$, alphacarotene, beta-carotene, lycopene, and lutein/zeaxanthin. The association of vitamin $E$ and HNC was modified by alcohol status ( $P$ interaction=0.003), with lower risks in alcohol abstainers.

\section{Conclusions}

This study shows an inverse association between intake of vitamin $C$ and the incidence of HNC (subtypes). Future research is recommended to investigate the underlying mechanisms and to confirm our results, which may be promising for the prevention of HNC. 


\section{Introduction}

Head and neck cancer (HNC) is the seventh most commonly occurring type of cancer worldwide and the seventh most common cause of death from all cancers. ${ }^{1,2}$ The estimated incidence worldwide is 600,000 people every year, with the highest incidence in males aged 60-74 year. ${ }^{1,3}$

Alcohol consumption, tobacco use and human papillomavirus infection (HPV) are identified as important risk factors for $\mathrm{HNC}^{2,4-9}$ An inverse association is implicated for fruit and vegetable intake and HNC risk, probably because they contain vitamins and carotenoids that exhibit antioxidant properties. ${ }^{2,10-12}$ Antioxidants can protect the human body against free radicals that are associated with a higher cancer risk. ${ }^{13-15}$ Besides, vitamins and carotenoids increase the apoptosis of cancer cells, decrease cell proliferation and maintain the normal DNA repair system. In addition, the nutrients are essential for normal cell differentiation. ${ }^{16}$

One prospective cohort study investigated the association between vitamins and carotenoids and the risk on HNC. ${ }^{17}$ Despite the small number of cases among the 34,691 postmenopausal women, the results suggest that a high intake of antioxidants may be protective against HNC. A meta-analysis from 2013 provided evidence about the protective effect of carotenoids on HNC risk. ${ }^{18}$ However, this study was mainly based on case-control studies. In addition, a pooled analysis from the INHANCE consortium, based on 12 case-control studies, provided evidence about the protective effect of vitamin $C$ supplement use. ${ }^{19}$ Finally, randomized controlled trials have shown inconsistent results between the association of vitamin $\mathrm{E}$ and beta-carotene supplementation and risk on HNC (subtypes). ${ }^{20,21}$ Thus, the results of epidemiological data are inconclusive and are mainly based on case-control studies.

Therefore, we studied the association of dietary intake of vitamins $C$ and $E$, the most common carotenoids (alpha-carotene, beta-carotene, lutein/zeaxanthin, lycopene, and beta-cryptoxanthin) and vitamin $C$ and $E$ supplements and the risk on HNC and HNC subtypes in a large prospective cohort study. The following hypotheses were investigated: 1) HNC risk is higher in participants with a low intake of vitamins and carotenoids; and 2) these risks are different for HNC subtypes, i.e., oral cavity cancer (OCC), oro-/hypopharyngeal cancer (OHPC), and laryngeal cancer (LC). Furthermore, we investigated the possible effect modification by smoking and alcohol consumption. 


\section{Methods}

\section{Study design and population}

The study characteristics have been described in detail previously. ${ }^{22}$ The Netherlands Cohort Study (NLCS) started in September 1986 and included 120,852 participants. The participants were aged 55-69 years at baseline and were derived from 204 Dutch municipal computerized population registries. Participants completed a questionnaire at baseline.

For efficiency reasons a case-cohort design was used. A total of 5,000 participants (2,411 men and 2,589 women) were randomly sampled from the total cohort at baseline to create a subcohort from which person-years at risk were estimated. Incident cases were derived from the entire cohort. All incident cases were monitored during the follow-up period of 20.3 years, through annual linkage to the Netherlands Cancer Registry and the Netherlands Pathology Registry. The completeness of this cancer follow-up is estimated to be $\geq 96 \%{ }^{23}$

Prevalent cancer cases at baseline were excluded from the analysis, except for skin cancer. Participants with incomplete and inconsistent dietary data and missing data on exposure or confounding variables were also excluded. Incomplete and inconsistent dietary data included 1) participants who left 60 or more out of 150 items blank and ate fewer than 35 items at least once per month and 2) participants who left one or more item blocks blank. ${ }^{24}$ In total, 415 pathologically confirmed incident cases and 3,898 subcohort members were available for this study (Figure 4.1). As proposed by Hashibe et al. ${ }^{25}, 131$ cases were classified as OCC, 88 as OHPC, and 193 cases were classified as LC. Furthermore, 3 cases were classified as oral cavity, pharynx unspecified, or overlapping.

Approval for the NLCS was obtained by the review boards of TNO Nutrition and Food Research Institute and Maastricht University. All participants were informed before participation.

\section{Questionnaire data}

At baseline, all participants completed a self-administered questionnaire, which purpose was to assess habitual food consumption, lifestyle, and other cancer risk factors preceding the start of the study.

The 150-item food frequency questionnaire (FFQ) was part of the baseline questionnaire. To make sure misclassification was minimized, an elaborate dietary assessment covering seasonal variations was necessary. The FFQ contained most fruits and vegetables (the main contributors of vitamins and carotenoids), which were eaten 


\begin{tabular}{|c|c|c|c|c|}
\hline \multicolumn{5}{|c|}{ Netherlands Cohort Study on diet and cancer $(120,852)$} \\
\hline$\downarrow$ & & & $\downarrow$ & \\
\hline $\begin{array}{l}\text { Subcohort, randomly } \\
\text { sampled from total cohort }\end{array}$ & \multicolumn{4}{|c|}{ Record linkage with Netherlands Cancer Registry and PALGA } \\
\hline$\stackrel{\downarrow}{\downarrow}$ & $\begin{array}{l}\downarrow \\
\text { HNC overall }\end{array}$ & $\begin{array}{l}\downarrow \\
\text { Oral cavity } \\
\text { cancer (OCC) }\end{array}$ & \begin{tabular}{l}
\multicolumn{1}{c}{$\downarrow$} \\
Oro-/hypopharyngeal \\
cancer (OHPC)
\end{tabular} & $\begin{array}{l}\downarrow \\
\text { Laryngeal } \\
\text { cancer (LC) }\end{array}$ \\
\hline \multicolumn{5}{|c|}{ Exclusion of participants with prevalent cancer at baseline } \\
\hline $\begin{array}{c}\downarrow \\
4,774\end{array}$ & $\begin{array}{c}\downarrow \\
515\end{array}$ & $\begin{array}{c}\downarrow \\
149\end{array}$ & $\begin{array}{c}\downarrow \\
109\end{array}$ & $\begin{array}{c}\downarrow \\
254\end{array}$ \\
\hline \multicolumn{5}{|c|}{ Exclusion of participants with incomplete or inconsistent dietary data } \\
\hline $\begin{array}{c}\downarrow \\
4,198\end{array}$ & $\begin{array}{c}\downarrow \\
455\end{array}$ & $\begin{array}{c}\downarrow \\
136\end{array}$ & $\begin{array}{l}\downarrow \\
98\end{array}$ & $\begin{array}{c}\downarrow \\
218\end{array}$ \\
\hline \multicolumn{5}{|c|}{ Exclusion of participants with missing data on confounders } \\
\hline $\begin{array}{c}\downarrow \\
3,898\end{array}$ & $\begin{array}{c}\downarrow \\
415\end{array}$ & $\begin{array}{c}\downarrow \\
131\end{array}$ & $\begin{array}{c}\downarrow \\
88\end{array}$ & $\begin{array}{c}\downarrow \\
193\end{array}$ \\
\hline
\end{tabular}

Figure 4.1. Flow diagram of the number of subcohort members and cases on whom the analyses were based. Abbreviation PALGA: nationwide network and registry of histopathology and cytopathology in the Netherlands.

${ }^{a}$ Oral cavity cancer; oro-/hypopharyngeal cancer; oral cavity, pharynx unspecified or overlapping cancer; laryngeal cancer.

regularly in 1986, except for red cabbage, cucumber and chicory. Since broccoli was a rarely available vegetable in 1986, it was also excluded from the FFQ. The frequency of vegetable intake was divided into categories ranging from 'never or less than once per month' to '3-7 times per week'. However, a distinction was made between the intake of vegetables in the summer and in the winter. In addition, the consumption frequency of fruit ranged from 'never or less than once per month' to '6-7 times per week'. In addition, participants could fill out the number of fruits they ate per consumption. For cooked endive and string beans, participants were asked about their usual serving sizes (tablespoons and grams per person). The mean of those two vegetables was used as a representative of standard serving sizes of all leafy and solid vegetables respectively because a pilot study showed a correlation between serving sizes of different types of cooked vegetables. The serving size was multiplied with a vegetable-specific factor, to determine the mean daily intake in grams for vegetable consumption. The FFQ also included the self-reported use of supplements (vitamin supplements, drops, or others) during the five years prior to completion of the questionnaire. The type of supplement, brand, years of intake, and dose per day were asked. To reduce observer bias in coding or interpretation, data of the questionnaire was key-entered for incident cases and subcohort members, blinded with respect to case/subcohort status.

To calculate the daily intake of the vitamins $C$ and $E$ the Dutch food composition table was used. ${ }^{26}$ The mean daily intake of alpha-carotene, beta-carotene, lutein + zeaxanthin, lycopene, and beta-cryptoxanthin was calculated with an alternative food 
composition table, which was completed with data from manufacturers and recent literature. ${ }^{11}$ Since most of these literature sources did not provide separate values for each of the carotenoids, lutein and zeaxanthin were taken together.

The FFQ was validated and tested for reproducibility. ${ }^{24,27}$ Pearson correlation coefficients between the FFQ and the nine-day diet record were moderate; 0.58 for vitamin C and 0.52 for vitamin A (retinol + beta-carotene). Data was not available for the carotenoids, because there was no database for carotenoids separately at that time. However, the correlation of fruit, which is the main contributor of betacryptoxanthin, was 0.6 and for most macronutrients the correlation was between 0.65 and 0.80 . The reproducibility study considered only a number of nutrients relevant at that time for cancer studies, e.g., vitamin A (retinol and beta-carotene) and vitamin C. The five-year reproducibility of vitamin $A$ and $C$ ranged from a Pearson correlation coefficient of 0.51 to 0.64 , and can be considered as moderate.

Furthermore, detailed questions were asked about alcohol consumption and tobacco use at different moments during several years preceding the start of the NLCS. ${ }^{28}$ Briefly, we asked for the alcohol consumption the year prior to completion of the questionnaire. In addition, we asked for alcohol consumption five years before baseline. The mean daily intake of ethanol (g/day) was calculated by using the Dutch food composition table. The questionnaire asked about smoking status, number of cigarettes smoked daily, and number of smoking years.

\section{Statistical analysis}

The Cox proportional hazards model was used to estimate age- and sex-adjusted incidence rate ratios (RR), multivariable-adjusted RRs and $95 \%$ confidence intervals $(95 \% \mathrm{Cl})$. Analyses were performed for the vitamins $\mathrm{C}$ and $\mathrm{E}$, the carotenoids alphacarotene, beta-carotene, lutein + zeaxanthin, lycopene, and beta-cryptoxanthin, and the use of any supplement containing vitamin $\mathrm{C}$ or $\mathrm{E}$.

Participants were categorized, depending on the sex-specific distribution in the subcohort, according to quartiles (Q) of intake of these nutrients (Q1 for the lowest and Q4 for the highest quartile). Furthermore, participants were categorized as users or non-users of supplements containing vitamin C or E. Continuous variables were constructed for the vitamins and carotenoids, based on the difference between percentiles 75 and 25 in the subcohort. The increment ranged from $50 \mathrm{mg} /$ day for vitamin C to $0.2 \mathrm{mg} /$ day for beta-cryptoxanthin.

Vitamin and carotenoid intake of HNC cases diagnosed within the first two years of follow-up $(N=33)$ were compared with the intake of HNC cases diagnosed later in follow-up $(N=382)$. Early symptoms of HNC could influence the intake of vitamins and 
carotenoids and therefore lead to reversed causation. Independent samples t-tests were performed to test for significant differences, because the normality assumption was still violated after transformation of the nutrient intakes. No differences were found between the intake of vitamins and carotenoids between the early cases and the cases diagnosed later in follow-up, with a $P$ value ranging from 0.98 for lycopene (SD: 0.16 , median value of 0.83 vs. $0.75 \mathrm{mg} /$ day respectively) to 0.23 for alphacarotene (SD: 0.09 , median value of 0.43 vs. $0.52 \mathrm{mg} /$ day, respectively). In addition, sensitivity analyses were performed in which the first two years of follow-up were excluded. No differences were found between the results for the period with exclusion of the first two years of follow-up and the total follow-up period (Supplemental Table 4.1). Therefore, the total follow-up period was included in the analyses.

Person-years at risk were calculated from baseline until time of diagnosis of HNC, loss to follow-up, end of follow-up, emigration, or death, whichever occurred first. Predefined confounders were sex; age (years); alcohol consumption (grams/day); cigarette smoking status (never/former/current), frequency ( $N /$ day), and duration (years). The variables smoking frequency and duration were centered, as proposed by Leffondré et al. ${ }^{29}$ The categorical variable family history of HNC and the continuous variables BMI; level of education; intake of fish, red meat, and meat products (all grams/day); non-occupational physical activity; and total daily energy intake were considered as potential confounders. ${ }^{2,30}$ Confounders had to change the RR of vitamin C or E (continuous variable) with HNC overall or any of the HNC subtypes with at least 10 percent, by using a backwards stepwise procedure. This resulted in a final model which contained the predefined confounders and total daily energy intake ( $\mathrm{kcal} / \mathrm{day}$ ).

The proportional hazard $(\mathrm{PH})$ assumption was tested using the scaled Schoenfeld residuals for every covariate. ${ }^{31}$ When the $\mathrm{PH}$ assumption was violated, a time-varying covariate was introduced into the model and tested for significance. Current smoking did not meet the PH assumption for all HNC overall analyses, with a significant timevarying covariate. It is plausible that the interaction with time can be explained by participants who stopped smoking during the follow-up period. Therefore, the timevarying covariate for current smoking was also added in the model for HNC subtypes. A more elaborate explanation about this time-varying covariate is described elsewhere. ${ }^{28}$

To determine the adjusted RR of one nutrient, the independent variable was mutually adjusted for the other nutrients. In addition, $P$ trend was calculated by using the quartiles as continuous terms for the independent variables in the Cox proportional hazards model. The Wald test was used to evaluate this trend.

To detect possible interaction by sex, smoking and alcohol consumption, a test for interaction was performed, using the interaction term in the Cox proportional hazards 
model and Wald test for HNC overall cases. Furthermore, stratified analyses were performed for smoking status (never/former/current) and alcohol consumption (0 g/day; >0-15 g/day; $>15 \mathrm{~g} /$ day).

All $P$ values were based on two-sided tests and were considered statistically significant if $P<0.05$. Analyses were performed using the STATA software package (STATA, version 11.1; Stata Corporation, College Station, Texas, USA).

\section{Results}

\section{Baseline characteristics}

Cases were more frequently men (77.1\%) than women (Table 4.1). Among subcohort members, almost half (49.3\%) of the participants were men. Compared with cases, the daily median intake of most vitamins and carotenoids was higher among subcohort members, e.g., the intake of vitamin $C$ of subcohort members was $96.1 \mathrm{mg} /$ day vs. $82.3 \mathrm{mg} /$ day for HNC overall. There were more users of vitamin E supplements among subcohort members (6.5\%) than HNC overall cases (5.5\%). However, the use of vitamin C supplements was similar among subcohort members and HNC overall cases $(10.6 \%)$.

HNC overall cases were more often current smokers (56.4\%) with a higher number of pack-years (34.1) than subcohort members (26.8\% current smokers and 22.7 packyears). Furthermore, with respect to alcohol use, subcohort members were more often abstainers and had a substantially lower daily ethanol intake than cases.

\section{Main analyses}

Age- and sex-adjusted analyses (Table 4.2) showed strong inverse associations, which were mostly less strong after multivariable-adjusted analyses (Table 4.3).

Vitamin C intake was associated with a significant age- and sex-adjusted decreased risk of HNC overall (RR for Q4 vs. Q1: $0.45,95 \% \mathrm{Cl} 0.34-0.61, P$ trend<0.001) and of the subtypes OCC (RR for Q4 vs. Q1: $0.43,95 \% \mathrm{Cl} 0.25-0.73, P$ trend=0.005), OHPC (RR for Q4 vs. Q1: $0.50,95 \% \mathrm{Cl} 0.29-0.87, P$ trend=0.02), and LC (RR for Q4 vs. Q1: $0.46,95 \% \mathrm{Cl}$ 0.29-0.96, $P$ trend<0.001). In multivariable-adjusted analyses, vitamin $C$ intake was still significantly associated with a decreased risk of HNC overall (RR for Q4 vs. Q1: 0.39, 95\% $\mathrm{Cl} 0.23-0.66, P$ trend<0.001), OCC (RR for Q4 vs. Q1: $0.35,95 \% \mathrm{Cl} 0.16-0.77, P$ trend=0.03) and OHPC (RR for Q4 vs. Q1: 0.29, 95\% Cl 0.12-0.67, $P$ trend=0.003). However, the association between vitamin C and LC was not statically significant in the multivariable-adjusted analyses. 
In age- and sex-adjusted analyses, the $P$ trend was only statistically significant for the association of beta-cryptoxanthin intake and HNC overall $(P$ trend $<0.001)$ and LC ( $P$ trend=0.002). No statistically significant associations were observed between intake of vitamin $\mathrm{E}$, alpha-carotene, beta-carotene, lutein + zeaxanthin, lycopene and overall risk of HNC and the subtypes OCC, OHPC, and LC in age- and sex-adjusted analyses as well as multivariable analyses. However, most associations were below unity. Furthermore, after multivariable-adjusted analyses, the $P$ trend was not statistically significant for the association between beta-cryptoxanthin intake and HNC overall and the subtypes.

Regarding the use of supplements containing vitamin $C$, the point estimations were mostly above 1 for users vs. non-users for HNC overall (RR: $1.18,95 \% \mathrm{Cl} 0.76$ 1.85), OCC (RR: 0.99, 95\% Cl 0.53-1.86), OHPC (RR: 1.53, 95\% Cl 0.66-3.59), and LC (RR: $1.21,95 \% \mathrm{Cl} 0.65-2.24)$, but none of those were significant. The intake of supplements containing vitamin $\mathrm{E}$ was inversely, however not significant, associated with HNC overall (RR: $0.89,95 \% \mathrm{Cl} 0.51-1.54$ ). In addition, there were no clear associations between vitamin E supplements and OCC (RR: 0.90, 95\% Cl 0.40-2.03), OHPC (RR: 0.87, 95\% $\mathrm{Cl} 0.28-2.72$ ), and LC (RR: $1.07,95 \% \mathrm{Cl} 0.47-2.43$ ). However, the number of participants using supplements was small, e.g., $N=44$ for vitamin $C$ and $N=23$ for vitamin E for HNC overall cases.

\section{Stratified analyses}

No statistically significant interaction was found between sex and the independent variables ( $P$ interaction ranging from 0.15 for alpha-carotene to 0.75 for lycopene) (Tables 4.4 and 4.5). Hence, analyses were performed for both sexes combined.

Statistically significant interactions were found between categories of alcohol consumption and vitamin $E$ intake ( $P$ interaction=0.003) (Table 4.5). Alcohol abstainers showed decreased risks and the group of participants who drank $\geq 15$ grams/day showed mostly increased risks compared with the reference group of participants who drank >0-15 grams/day. In alcohol abstainers a statistically significant inverse association between vitamin $\mathrm{E}$ intake and HNC risk were found in Q3 (RR: 0.29, 95\% Cl 0.10-0.85) and Q4 (RR: $0.15,95 \% \mathrm{Cl} 0.03-0.64)$. In participants who drank $>15$ grams of alcohol per day, statistically significantly associations between vitamin $E$ intake and HNC risk were found in Q1 (RR: 1.97, 95\% $\mathrm{Cl} 1.26-3.09$ ) and Q2 (RR: 1.77, 95\% Cl 1.092.88). Furthermore, when stratified on smoking status, most results were below unity for never smokers and showed increased risks for current smokers, compared with never smokers in Q1. 


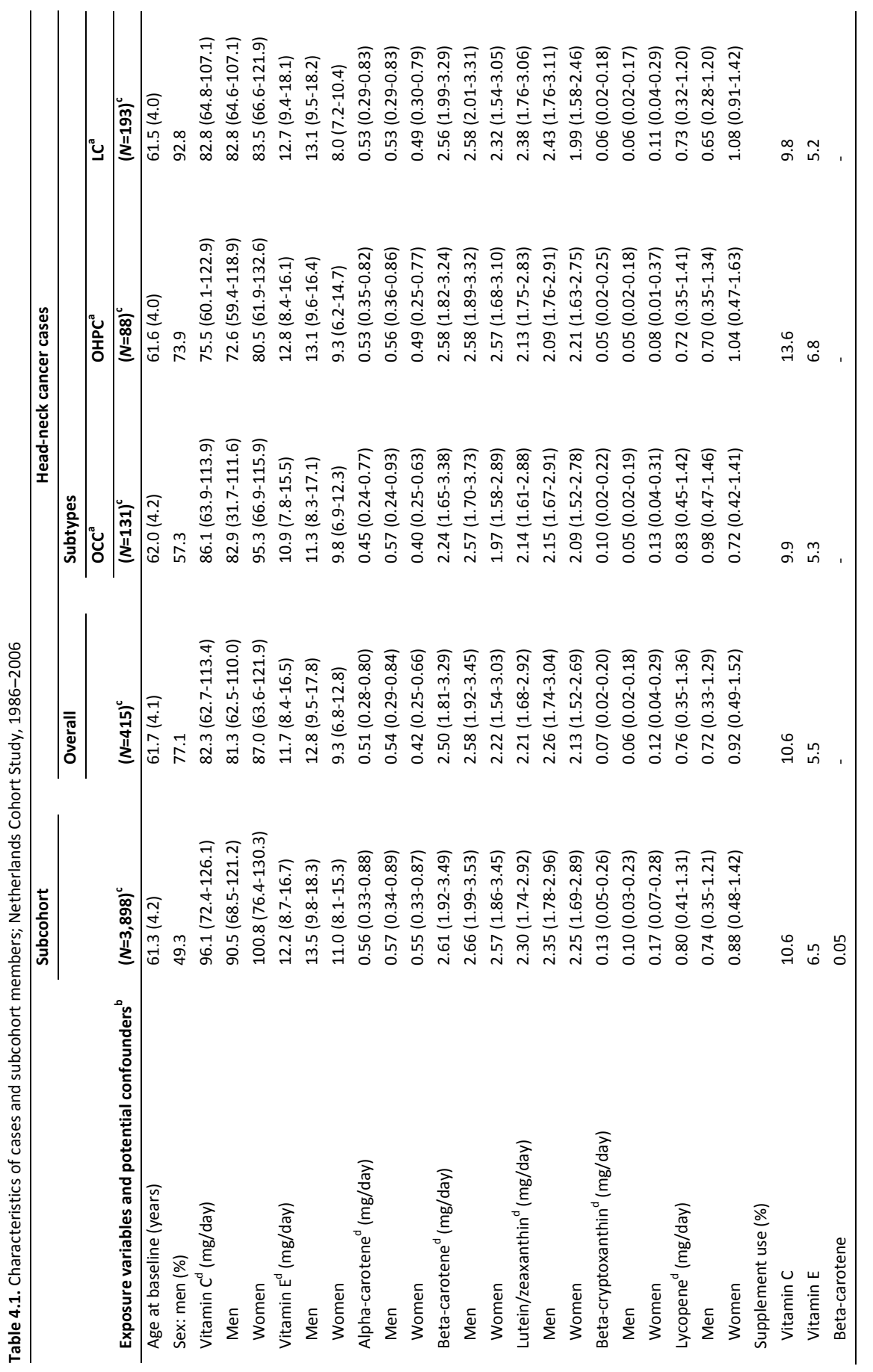




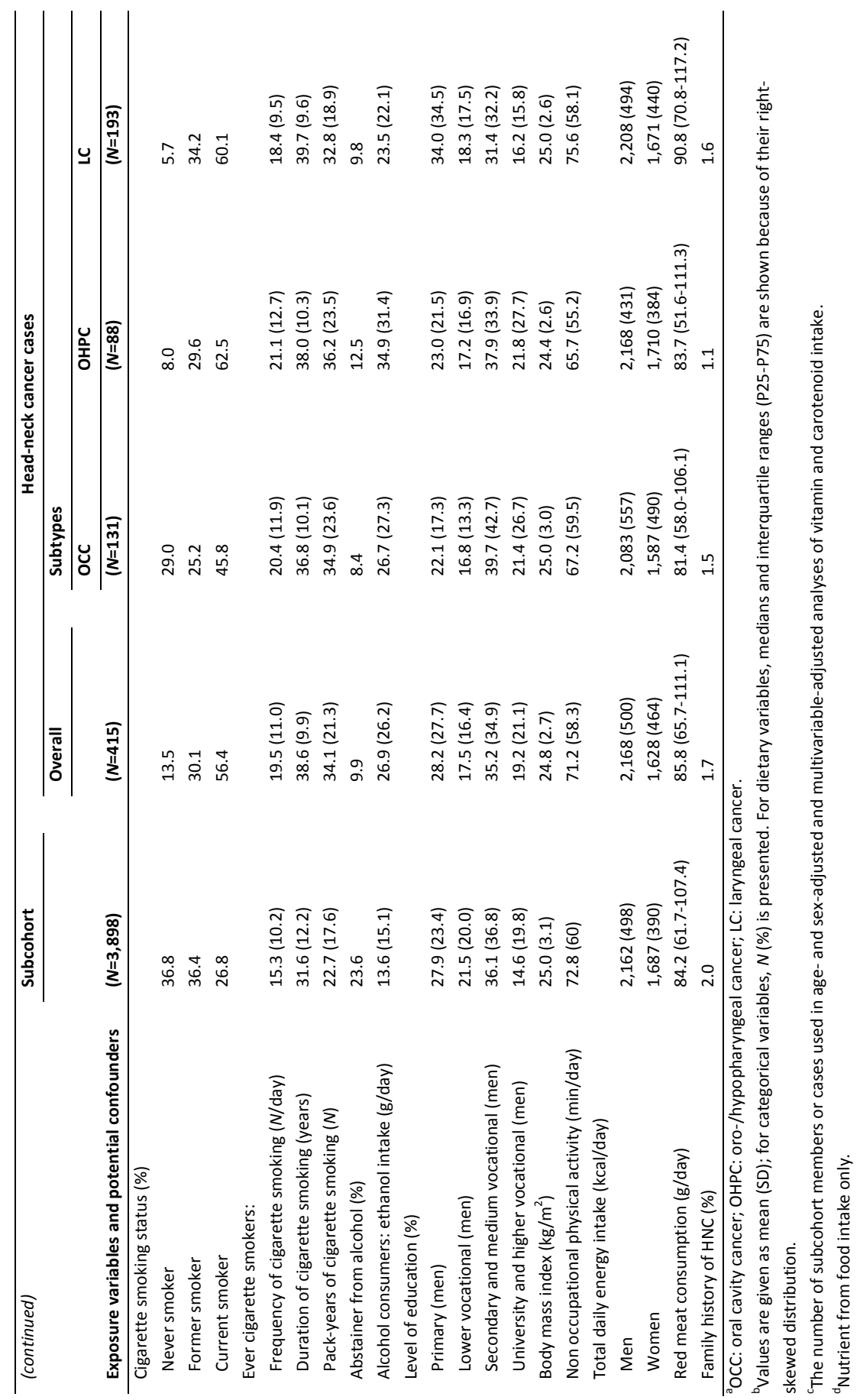




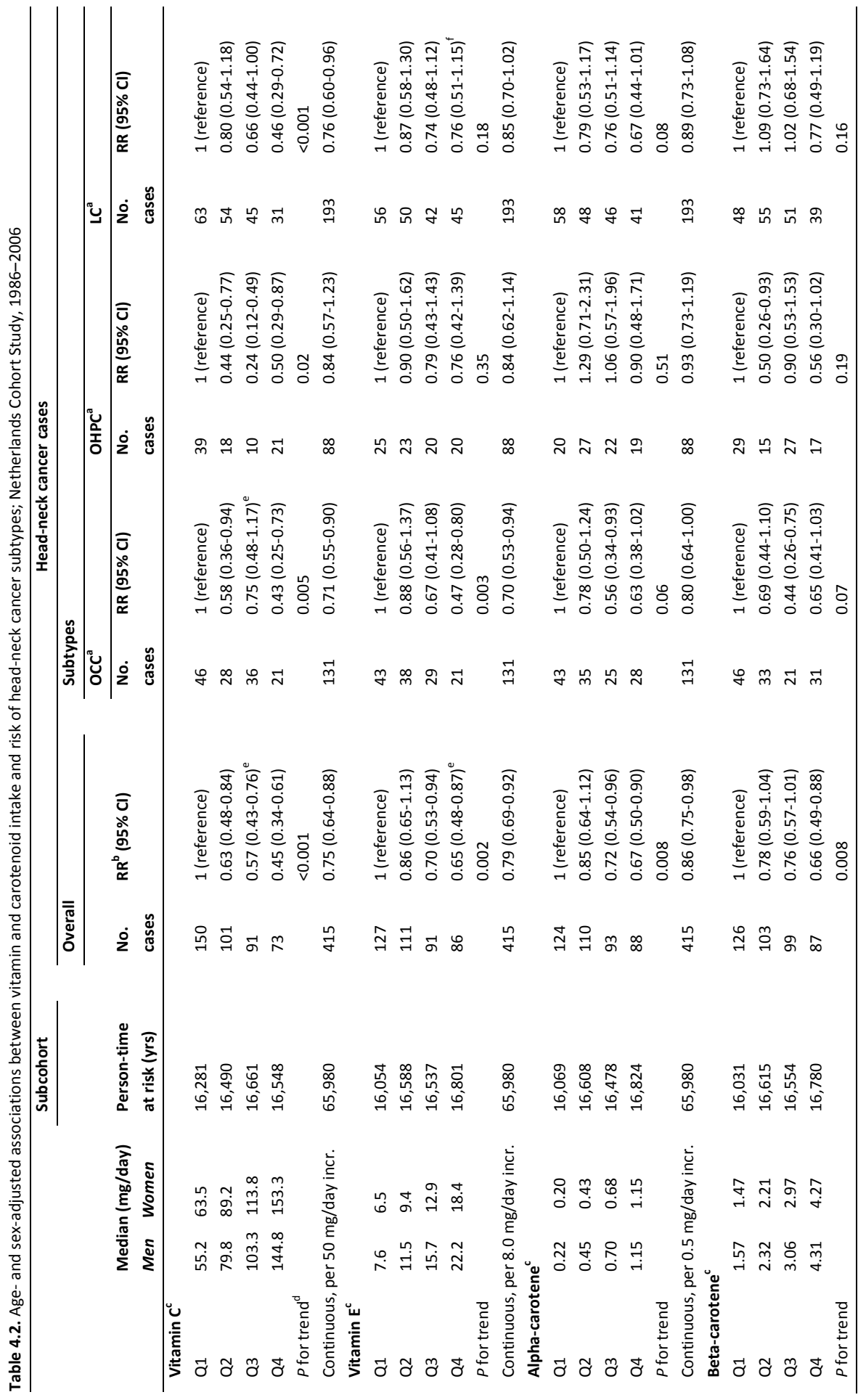




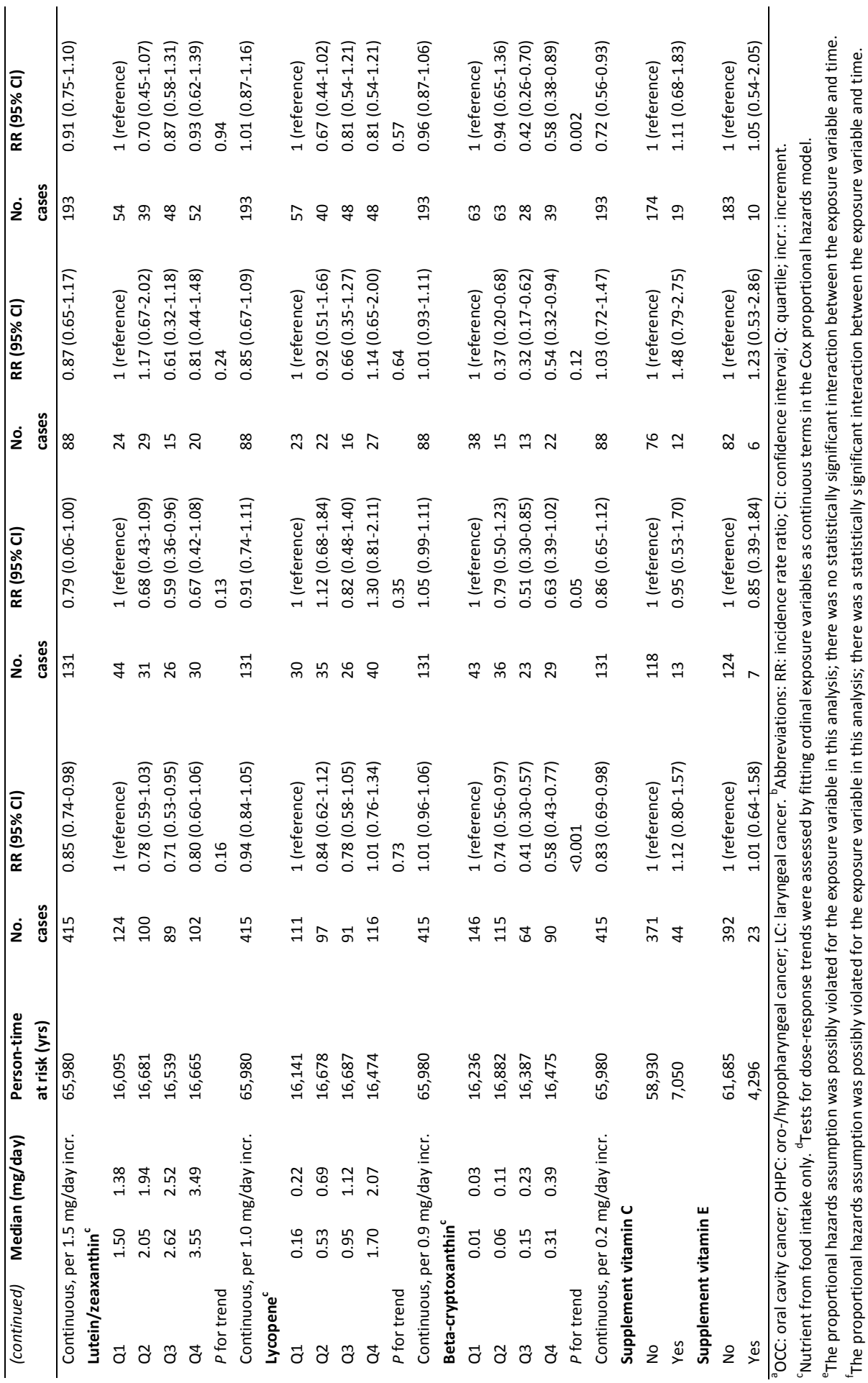




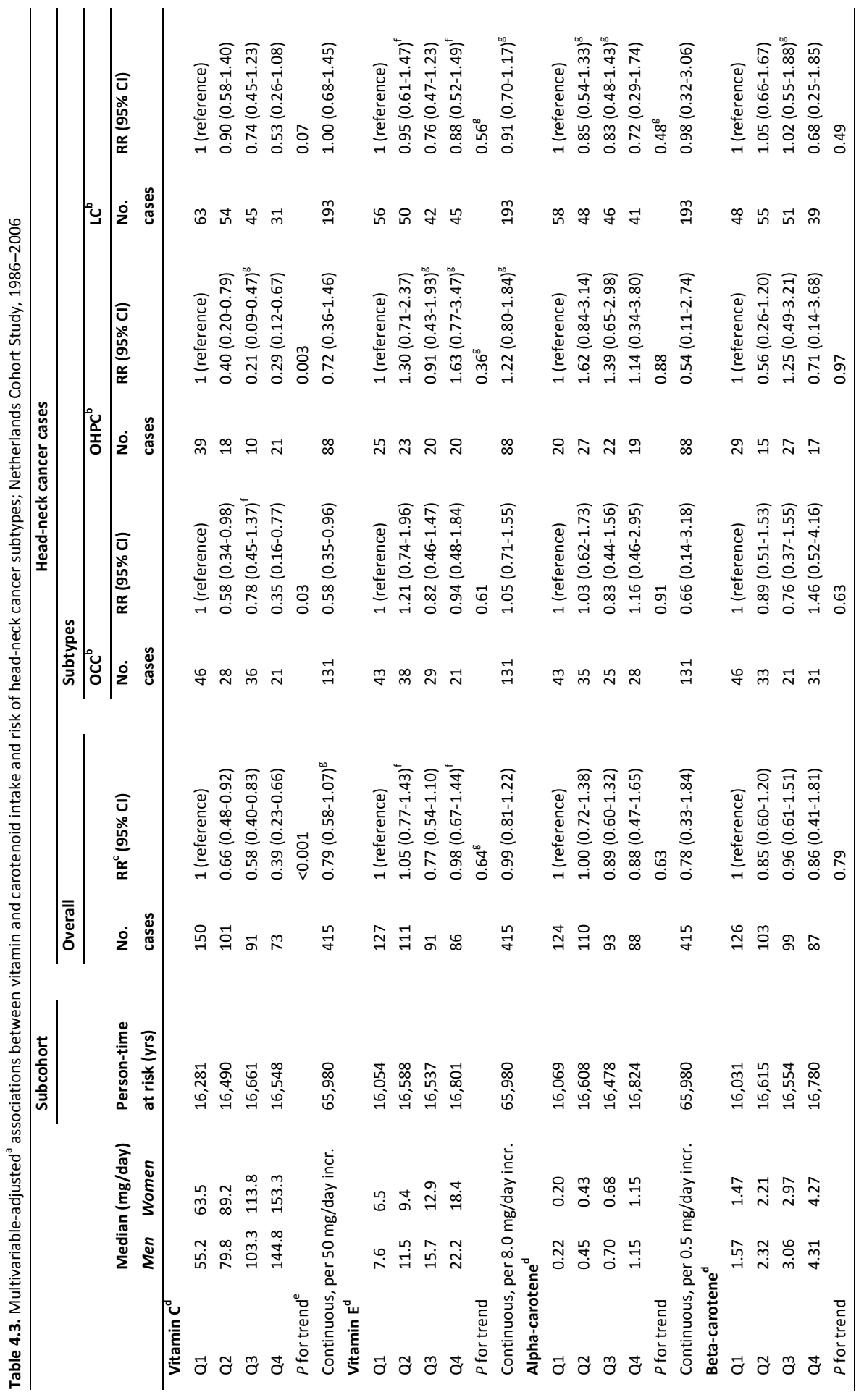




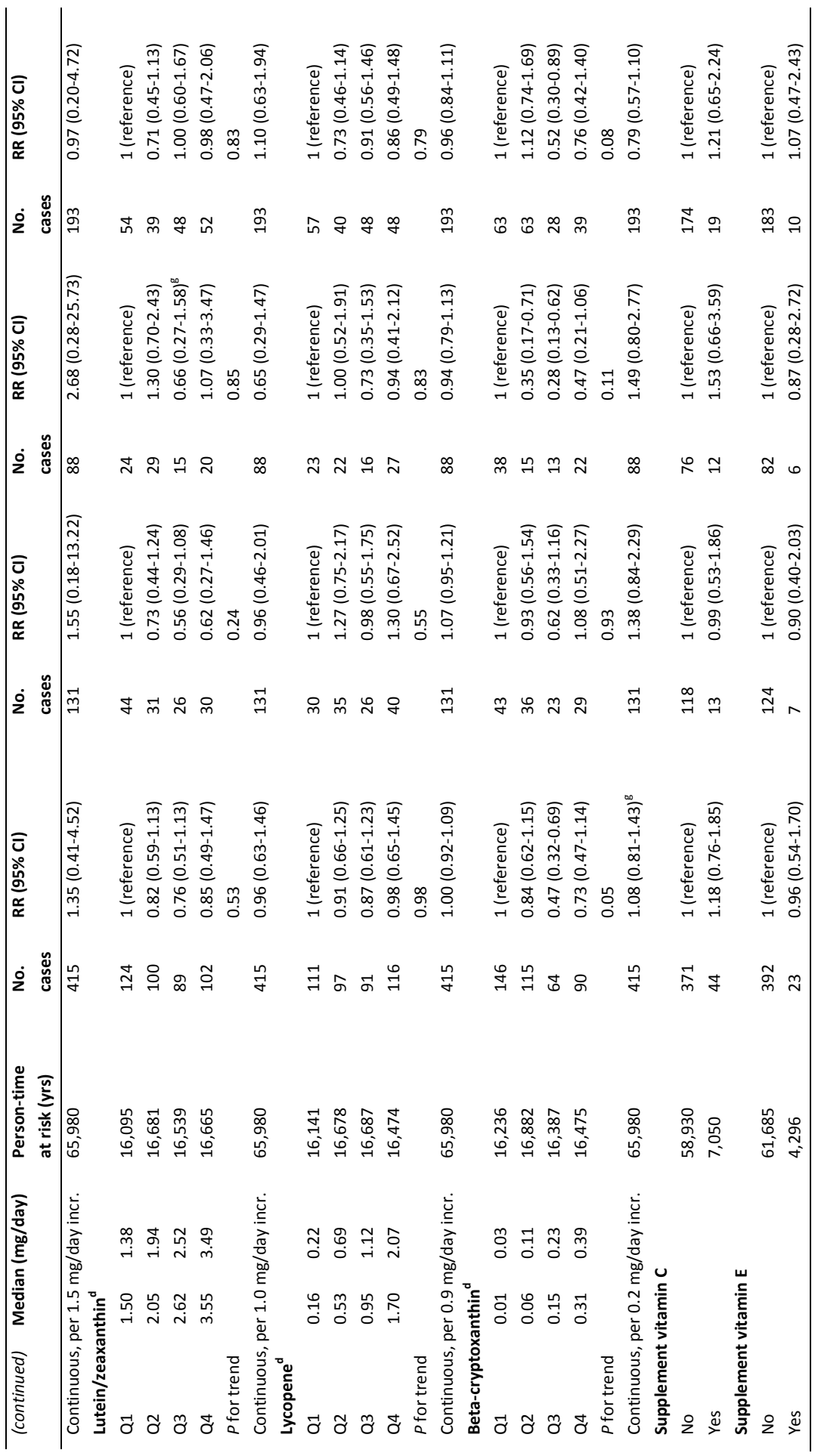




\section{Table 4.3 (footnotes).}

${ }^{a}$ Mutually adjusted for age (years); sex; cigarette smoking (status (never/former/current), frequency (number of cigarettes per day; continuous, centered), duration (number of years; continuous, centered)); alcohol consumption (grams ethanol per day; continuous); and total daily energy intake (kcal/day; continuous).

${ }^{b}$ OCC: oral cavity cancer; OHPC: oro-/hypopharyngeal cancer; LC: laryngeal cancer.

${ }^{\mathrm{C}}$ Abbreviations: RR: incidence rate ratio; $\mathrm{Cl}$ : confidence interval; Q: quartile; incr.: increment.

${ }^{\mathrm{d}}$ Nutrient from food intake only.

${ }^{\mathrm{e}}$ Tests for dose-response trends were assessed by fitting ordinal exposure variables as continuous terms in the Cox proportional hazards model.

${ }^{\mathrm{f}}$ The proportional hazards assumption was possibly violated for the exposure variable in this analysis; there was a statistically significant interaction between the exposure variable and time.

${ }^{\mathrm{g}}$ The proportional hazards assumption was possibly violated for the exposure variable in this analysis; there was no statistically significant interaction between the exposure variable and time. 
Table 4.4. Stratified analyses of sex/cigarette smoking/alcohol consumption and quartiles of vitamin $\mathrm{C}$ intake and risk (multivariable-adjusted ${ }^{a}$ associations) of head-neck cancer overall; Netherlands Cohort Study, 1986-2006

\begin{tabular}{|c|c|c|c|c|}
\hline \multicolumn{5}{|c|}{ Head-neck cancer overall } \\
\hline & \multicolumn{4}{|c|}{ Vitamin C intake } \\
\hline & $\mathrm{Q1}^{\mathrm{b}}$ & Q2 & Q3 & Q4 \\
\hline \multicolumn{5}{|l|}{ Sex } \\
\hline \multicolumn{5}{|l|}{ M } \\
\hline Cases/person-time at risk (years) & $110 / 7,373$ & $84 / 7,747$ & $72 / 7,868$ & $54 / 7,682$ \\
\hline $\mathrm{RR}(95 \% \mathrm{Cl})$ & 1 (ref) & $0.75(0.52-1.10)$ & $0.63(0.42-0.94)$ & $0.40(0.22-0.71)$ \\
\hline \multicolumn{5}{|l|}{$\mathrm{F}$} \\
\hline Cases/person-time at risk (years) & $40 / 8,909$ & $17 / 8,743$ & $19 / 8,793$ & $19 / 8,867$ \\
\hline $\mathrm{RR}(95 \% \mathrm{Cl})$ & $0.54(0.34-0.86)$ & $0.22(0.12-0.41)$ & $0.23(0.13-0.42)$ & $0.19(0.10-0.38)$ \\
\hline$P$ for interaction ${ }^{c}$ & 0.34 & & & \\
\hline \multicolumn{5}{|l|}{ Cigarette smoking } \\
\hline \multicolumn{5}{|l|}{ Never } \\
\hline Cases/person-time at risk (years) & $19 / 6,130$ & $10 / 6,595$ & $15 / 6,080$ & $12 / 7,029$ \\
\hline $\mathrm{RR}(95 \% \mathrm{Cl})$ & 1 (reference) & $0.45(0.21-1.00)$ & $0.71(0.34-1.47)$ & $0.37(0.15-0.90)$ \\
\hline \multicolumn{5}{|l|}{ Former } \\
\hline Cases/person-time at risk (years) & $36 / 5,320$ & $27 / 5,889$ & $33 / 6,386$ & $29 / 6,127$ \\
\hline $\mathrm{RR}(95 \% \mathrm{Cl})$ & $1.25(0.67-2.31)$ & $0.79(0.41-1.53)$ & $0.85(0.44-1.64)$ & $0.64(0.30-1.34)$ \\
\hline \multicolumn{5}{|l|}{ Current } \\
\hline Cases/person-time at risk (years) & $95 / 4,832$ & $64 / 4,006$ & $43 / 4,195$ & $32 / 3,392$ \\
\hline $\mathrm{RR}(95 \% \mathrm{Cl})$ & $2.71(1.53-4.80)$ & $2.02(1.11-3.66)$ & $1.34(0.71-2.53)$ & $0.93(0.44-1.98)$ \\
\hline$P$ for interaction & 0.53 & & & \\
\hline \multicolumn{5}{|l|}{ Alcohol consumption } \\
\hline \multicolumn{5}{|l|}{ Abstainers } \\
\hline Cases/person-time at risk (years) & $14 / 4,382$ & $10 / 3,768$ & $5 / 3,449$ & $12 / 3,810$ \\
\hline $\mathrm{RR}(95 \% \mathrm{Cl})$ & $0.62(0.32-1.19)$ & $0.56(0.27-1.16)$ & $0.25(0.09-0.67)$ & $0.45(0.21-0.97)$ \\
\hline \multicolumn{5}{|l|}{$>0-15$ (g/day) } \\
\hline Cases/person-time at risk (years) & $48 / 8,223$ & $37 / 8,923$ & $46 / 8,852$ & $28 / 8,415$ \\
\hline $\mathrm{RR}(95 \% \mathrm{Cl})$ & 1 (reference) & $0.68(0.42-1.10)$ & $0.89(0.55-1.44)$ & $0.50(0.26-0.98)$ \\
\hline \multicolumn{5}{|l|}{$\geq 15$ (g/day) } \\
\hline Cases/person-time at risk (years) & $88 / 3,677$ & $54 / 3,799$ & $40 / 4,360$ & $33 / 4,323$ \\
\hline $\mathrm{RR}(95 \% \mathrm{Cl})$ & $2.16(1.42-3.28)$ & $1.42(0.89-2.25)$ & $0.87(0.52-1.45)$ & $0.69(0.38-1.24)$ \\
\hline$P$ for interaction & 0.08 & & & \\
\hline
\end{tabular}

${ }^{a}$ Mutually adjusted for age (years); sex; cigarette smoking (status (never/former/current), frequency (number of cigarettes per day; continuous, centered), duration (number of years; continuous, centered)); alcohol consumption (grams ethanol per day; continuous); and total daily energy intake (kcal/day; continuous).

${ }^{\mathrm{b}}$ Abbreviations: RR: incidence rate ratio; $\mathrm{Cl}$ : confidence interval; Q: quartile.

${ }^{\mathrm{C}} P$ value for interaction based on cross-product terms in the Cox proportional hazards model and Wald test. 
Table 4.5. Stratified analyses of sex/cigarette smoking/alcohol consumption and quartiles of vitamin $E$ intake and risk (multivariable-adjusted ${ }^{\text {a }}$ associations) of head-neck cancer overall; Netherlands Cohort Study, 1986-2006

\begin{tabular}{|c|c|c|c|c|}
\hline \multicolumn{5}{|c|}{ Head-neck cancer overall } \\
\hline & \multicolumn{4}{|c|}{ Vitamin E intake } \\
\hline & $\mathrm{Q1}^{\mathrm{b}}$ & Q2 & Q3 & Q4 \\
\hline \multicolumn{5}{|l|}{ Sex } \\
\hline \multicolumn{5}{|l|}{ M } \\
\hline Cases/person-time at risk (years) & $91 / 7,413$ & $84 / 7,733$ & $73 / 7,628$ & $72 / 7,896$ \\
\hline $\mathrm{RR}(95 \% \mathrm{Cl})$ & 1 (reference) & $1.12(0.78-1.61)$ & $0.87(0.57-1.32)$ & $1.16(0.75-1.80)$ \\
\hline \multicolumn{5}{|l|}{$\mathrm{F}$} \\
\hline Cases/person-time at risk (years) & $36 / 8,641$ & $27 / 8,856$ & $18 / 8,909$ & $14 / 8,905$ \\
\hline $\mathrm{RR}(95 \% \mathrm{Cl})$ & $0.60(0.38-0.95)$ & $0.54(0.33-0.90)$ & $0.35(0.19-0.62)$ & $0.34(0.18-0.64)$ \\
\hline$P$ for interaction ${ }^{c}$ & 0.27 & & & \\
\hline \multicolumn{5}{|l|}{ Cigarette smoking } \\
\hline \multicolumn{5}{|l|}{ Never } \\
\hline Cases/person-time at risk (years) & $23 / 5,996$ & $15 / 6,541$ & $9 / 6,325$ & $9 / 6,972$ \\
\hline $\mathrm{RR}(95 \% \mathrm{Cl})$ & 1 (reference) & $0.67(0.34-1.34)$ & $0.43(0.19-0.96)$ & $0.44(0.20-0.98)$ \\
\hline \multicolumn{5}{|l|}{ Former } \\
\hline Cases/person-time at risk (years) & $26 / 5,666$ & $33 / 6,112$ & $32 / 5,921$ & $34 / 6,023$ \\
\hline $\mathrm{RR}(95 \% \mathrm{Cl})$ & $0.63(0.33-1.18)$ & $0.99(0.54-1.81)$ & $0.94(0.51-1.74)$ & $1.17(0.62-2.20)$ \\
\hline \multicolumn{5}{|l|}{ Current } \\
\hline Cases/person-time at risk (years) & $78 / 4,391$ & $63 / 3,936$ & $50 / 4,290$ & $43 / 3,806$ \\
\hline $\mathrm{RR}(95 \% \mathrm{Cl})$ & $2.04(1.18-3.53)$ & $2.03(1.14-3.63)$ & $1.34(0.71-2.51)$ & $1.76(0.92-3.35)$ \\
\hline$P$ for interaction & 0.06 & & & \\
\hline \multicolumn{5}{|l|}{ Alcohol consumption } \\
\hline \multicolumn{5}{|l|}{ Abstainers } \\
\hline Cases/person-time at risk (years) & $15 / 4,497$ & $20 / 3,968$ & $4 / 3,313$ & $2 / 3,630$ \\
\hline $\mathrm{RR}(95 \% \mathrm{Cl})$ & $0.62(0.32-1.18)$ & $1.42(0.77-2.61)$ & $0.29(0.10-0.85)$ & $0.15(0.03-0.64)$ \\
\hline \multicolumn{5}{|l|}{$>0-15$ (g/day) } \\
\hline Cases/person-time at risk (years) & $37 / 7,414$ & $37 / 8,751$ & $37 / 8,918$ & $48 / 9,330$ \\
\hline $\mathrm{RR}(95 \% \mathrm{Cl})$ & 1 (reference) & $0.86(0.52-1.40)$ & $0.91(0.55-1.50)$ & $1.23(0.74-2.05)$ \\
\hline \multicolumn{5}{|l|}{$\geq 15$ (g/day) } \\
\hline Cases/person-time at risk (years) & $75 / 4,143$ & $54 / 3,870$ & $50 / 4,305$ & $36 / 3,841$ \\
\hline $\mathrm{RR}(95 \% \mathrm{Cl})$ & $1.97(1.26-3.09)$ & $1.77(1.09-2.88)$ & $1.46(0.87-2.45)$ & $1.26(0.71-2.24)$ \\
\hline$P$ for interaction & 0.003 & & & \\
\hline
\end{tabular}

${ }^{a}$ Mutually adjusted for age (years); sex; cigarette smoking (status (never/former/current), frequency (number of cigarettes per day; continuous, centered), duration (number of years; continuous, centered)); alcohol consumption (grams ethanol per day; continuous); and total daily energy intake (kcal/day; continuous).

${ }^{\mathrm{b}}$ Abbreviations: RR: incidence rate ratio; $\mathrm{Cl}$ : confidence interval; Q: quartile.

${ }^{\mathrm{C}} P$ value for interaction based on cross-product terms in the Cox proportional hazards model and Wald test. 


\section{Discussion}

This study examined associations of vitamin and carotenoid intake with HNC risk. Strong inverse associations were found between vitamin C and HNC overall, OCC, and OHPC. No clear associations were found for other nutrients and vitamin supplementation; however, most point estimates showed possible protective effects. In addition, no clear differences were found between the associations of vitamin and carotenoid intake and the risk on subtypes of HNC. Furthermore, the association between vitamin $\mathrm{E}$ intake and the risk on HNC overall was modified by alcohol consumption.

\section{Vitamin and carotenoid intake}

To our knowledge, this is the largest prospective study to investigate the association of vitamin and carotenoid intake and the risk on HNC, including the most common subtypes OCC, OHCP, and LC. The lowa Women's Health Study (IWHS) reported a relative risk of 0.7 (95\% $\mathrm{Cl} 0.3-1.7)$ for vitamin $\mathrm{C}$, comparing tertile 3 vs. tertile $1 .^{17} \mathrm{~A}$ stronger effect is found in our study, possibly due to the fact that quartiles result in more contrast between the groups compared with tertiles. A nested case-control study suggested a protective effect of carotenoid intake on oral cancer. However, only 28 cases were diagnosed with primary oral and pharyngeal cancer. ${ }^{32}$ Thus, evidence from prospective cohort studies is scarce, but supports our results.

The INHANCE consortium suggested that vitamin $C$ supplementation reduces the risk of HNC, but no linear trend was observed. ${ }^{33}$ A pooled analysis provided the same results as our study; no clear associations were found between supplementation and the risk on $\mathrm{HNC}^{34}$ The different associations of vitamin supplements and vitamin intake from food could be explained by the small sample size of supplement users. Furthermore, vitamin supplements could have other properties than vitamin intake through food.

A number of case control studies investigated vitamin and carotenoid intake and the risk on $\mathrm{HNC}^{35-38}$ A case-control report of Negri et al. ${ }^{37}$ reported an odds ratio (OR) of 0.34 ( $95 \% \mathrm{Cl} 0.23-0.51$ ) for vitamin C comparing quintile 5 vs. quintile 1 and oral and pharyngeal cancer. Furthermore, Negri et al. found significant protective associations for vitamin E (OR: $0.44,95 \% \mathrm{Cl} 0.28-0.71$ ) and carotene (OR: $0.43,95 \% \mathrm{Cl} 0.28-0.66$ ) intake, comparing the highest quintile with the lowest quintile. ${ }^{37}$ The differences with our study could be explained by the different study design, since recall bias, selection bias and reversed causation are more likely to occur in a case-control design or 
because of hospital-based controls, which may not be representative of the general population.

Overall, vitamin $\mathrm{C}$ is suggested to have a stronger inverse association with HNC than with other micronutrients. However, epidemiological evidence for other micronutrients remains inconclusive. In addition, evidence from case-control studies regarding the micro-nutrients and HNC risk provides inconsistent results.

The association of vitamin $C$ intake in our study seems to be stronger for OCC compared with OHPC and LC. The results of other studies are inconsistent, and therefore, no conclusion could be drawn. However, because the intake of carotenoids and vitamins is highly dependent on the intake of fruit and vegetables ${ }^{11}$, results could be compared to associations of fruit and vegetable intake. A large prospective cohort study for the association between fruit and vegetables intake and HNC subtypes confirms that there is a stronger benefit for OCC and OHPC with a high intake, compared with LC. ${ }^{10,12}$

\section{Interaction between alcohol and smoking and intake of vitamins and carotenoids}

No clear differences in associations with HNC were found among strata of cigarette smoking. The INHANCE consortium ${ }^{33}$ showed no significant associations for supplement use of vitamin $\mathrm{C}$ or $\mathrm{E}$ when stratified by smoking or drinking status. However, due to the small number of subjects in that group and therefore a lack of power, conclusions cannot be drawn. We found differences in the risk estimates among strata of smoking and alcohol use. It is plausible that alcohol consumption and smoking status would modify the association between the intake of vitamins and carotenoids and HNC. Oxidative stress could be caused by tobacco or alcohol use, which can be prevented by the effect of vitamins. 39,40

Thus, only one other study investigated interaction effects of cigarette smoking and alcohol consumption. ${ }^{33}$ Because of low number of participants in the strata, evidence about potential interaction effects on HNC risk remains inconclusive.

\section{Mechanisms}

The exact mechanisms of vitamins and carotenoids with regard to cancer risk are not fully known. ${ }^{41}$ It is suggested that they play a protective role in cancer and cardiovascular diseases. ${ }^{42-44}$ Deficiency of micronutrients can damage DNA ${ }^{45}$ and vitamin $\mathrm{C}$ might play a role in the chemoprevention of cancer; the micronutrient blocks metabolic activation of carcinogens, prevents oxidative stress and stimulates the immune function. ${ }^{39}$ Furthermore, vitamin $E$ helps to detoxify drugs and other toxins 
that promote the development of oxidative stress. ${ }^{40}$ Vitamin A, which is produced by alpha- and beta-carotene, induces differentiation and apoptosis. ${ }^{46}$

Differences in associations among HNC subtypes could be explained by different exposures due to the location of the tumor, as supposed by the WCRF. ${ }^{2}$ The mouth and pharynx are directly exposed to vitamin and carotenoid intake, which could lead to stronger inverse effects, compared with cancer of the larynx.

In conclusion, there is evidence about the protective effect for micronutrients regarding cancer; however, this evidence remains inconclusive and needs more clarification.

\section{Strengths and limitations}

An important strength of the NLCS is the prospective design, in which exposures were assessed before diagnosis. Therefore, it is not likely that recall bias or reversed causation influenced the results, which is confirmed by sensitivity analyses. Second, the NLCS makes it possible to distinguish between the most common HNC subtypes, due to the large number of participants. In addition, effect modification of alcohol consumption and smoking status could be investigated. Besides, tobacco smoking and alcohol use are elaborately measured in the questionnaire, which makes the occurrence of residual confounding for these variables unlikely. This is also confirmed by comparing the age- and sex-adjusted analyses with the multivariable-adjusted analyses. Furthermore, it is unlikely that loss to follow-up bias occurred, because of the high completeness of follow-up of both subcohort members and cases.

There are some limitations to this study. First, misclassification could have occurred for the exposure variables. If so, we expect it to be non-differential and could cause an underestimation of the effects. However, the participants had steady diet habits and the validation and reproducibility study showed that vitamins and carotenoids could rank subjects properly according to intake of the nutrients. ${ }^{24,27}$ Second, literature has shown that HPV is associated with an increased HNC risk. ${ }^{5}$ The questionnaire did not provide any information about HPV infections of the participants. This could have led to biased results. Third, proportional hazard assumptions were not fulfilled for some analyses. However, because of the limited number of cases we could not investigate this into depth.

\section{Conclusions}

This large and long-term prospective cohort study shows an inverse association between intake of vitamin C and the incidence of HNC (subtypes) and indicates an inverse association between the intake of vitamin $\mathrm{E}$ and carotenoids and the incidence 
of HNC and HNC subtypes. Future research is recommended to confirm our results and further investigate the underlying mechanisms, which may be promising for the prevention of HNC.

\section{References}

1 Ferlay, J. et al. GLOBOCAN 2012 v1.0, Cancer Incidence and Mortality Worldwide: IARC CancerBase No. 11. Lyon, France: International Agency for Research on Cancer. Available from: http://globocan.iarc.fr/Default.aspx, 2013.

2 World Cancer Research Fund / American Institute for Cancer Research. Food, Nutrition, Physical Activity and the Prevention of Cancer: a Global Perspective. American Institute for Cancer Research, 2007.

3 Integraal Kankercentrum Nederland. Netherlands Cancer Registry: Dutch Cancer Figures. Available from: www.cijfersoverkanker.nl, 2014.

4 Blot, W. J. et al. Smoking and drinking in relation to oral and pharyngeal cancer. Cancer Res. 48, 3282-3287 (1988).

5 Gillison, M. L. et al. Distinct risk factor profiles for human papillomavirus type 16-positive and human papillomavirus type 16-negative head and neck cancers. J. Natl. Cancer Inst. 100, 407-420 (2008).

6 Lewin, F. et al. Smoking tobacco, oral snuff, and alcohol in the etiology of squamous cell carcinoma of the head and neck: a population-based case-referent study in Sweden. Cancer 82, 1367-1375 (1998).

7 Soerjomataram, I., de Vries, E., Pukkala, E. \& Coebergh, J. W. Excess of cancers in Europe: a study of eleven major cancers amenable to lifestyle change. Int. J. Cancer 120, 1336-1343 (2007).

8 Sturgis, E. M., Wei, Q. \& Spitz, M. R. Descriptive epidemiology and risk factors for head and neck cancer. Semin. Oncol. 31, 726-733 (2004).

9 Talamini, R. et al. Combined effect of tobacco and alcohol on laryngeal cancer risk: a case-control study. Cancer Causes Control 13, 957-964 (2002).

10 Freedman, N. D. et al. Fruit and vegetable intake and head and neck cancer risk in a large United States prospective cohort study. Int J Cancer 122, 2330-2336 (2008).

11 Goldbohm, R. A., Brants, H. A., Hulshof, K. F. \& van den Brandt, P. A. The contribution of various foods to intake of vitamin A and carotenoids in The Netherlands. Int. J. Vitam. Nutr. Res. 68, 378-383 (1998).

12 Maasland, D. H., van den Brandt, P. A., Kremer, B., Goldbohm, R. A. \& Schouten, L. J. Consumption of vegetables and fruits and risk of subtypes of head-neck cancer in the Netherlands Cohort Study. Int J Cancer 136, E396-409 (2015).

13 El-Agamey, A. et al. Carotenoid radical chemistry and antioxidant/pro-oxidant properties. Arch. Biochem. Biophys. 430, 37-48 (2004).

14 Pham-Huy, L. A., He, H. \& Pham-Huy, C. Free radicals, antioxidants in disease and health. International journal of biomedical science : IJBS 4, 89-96 (2008).

15 Schoder, H. in Nuclear oncology: pathophysiology and clinical applications (ed H.W. Strauss), 269-295. Springer, 2013).

16 Steinmetz, K. A. \& Potter, J. D. Vegetables, fruit, and cancer prevention: a review. J. Am. Diet. Assoc. 96, 1027-1039 (1996).

17 Zheng, W. et al. Retinol, antioxidant vitamins, and cancers of the upper digestive tract in a prospective cohort study of postmenopausal women. Am J Epidemiol 142, 955-960 (1995).

18 Ge, X. X., Xing, M. Y., Yu, L. F. \& Shen, P. Carotenoid intake and esophageal cancer risk: a meta-analysis. Asian Pacific journal of cancer prevention : APJCP 14, 1911-1918 (2013).

19 Chuang, S. C. et al. Diet and the risk of head and neck cancer: a pooled analysis in the INHANCE consortium. Cancer Causes Control 23, 69-88 (2012).

20 Mayne, S. T. et al. Randomized trial of supplemental beta-carotene to prevent second head and neck cancer. Cancer Res. 61, 1457-1463 (2001).

21 Wright, M. E. et al. Effects of alpha-tocopherol and beta-carotene supplementation on upper aerodigestive tract cancers in a large, randomized controlled trial. Cancer 109, 891-898 (2007).

22 van den Brandt, P. A. et al. A large-scale prospective cohort study on diet and cancer in The Netherlands. J Clin Epidemiol 43, 285-295 (1990). 
23 Goldbohm, R. A., Van den Brandt, P. A. \& Dorant, E. Estimation of the coverage of Dutch municipalities by cancer registries and PALGA based on hospital discharge data. Tijdschr Soc Gezondheidsz 72, 80-84 (1994).

24 Goldbohm, R. A. et al. Validation of a dietary questionnaire used in a large-scale prospective cohort study on diet and cancer. Eur J Clin Nutr 48, 253-265 (1994).

25 Hashibe, M. et al. Alcohol drinking in never users of tobacco, cigarette smoking in never drinkers, and the risk of head and neck cancer: pooled analysis in the International Head and Neck Cancer Epidemiology Consortium. J. Natl. Cancer Inst. 99, 777-789 (2007).

26 Boffetta, P. \& Hashibe, M. Alcohol and cancer. Lancet Oncol 7, 149-156 (2006).

27 Goldbohm, R. A. et al. Reproducibility of a food frequency questionnaire and stability of dietary habits determined from five annually repeated measurements. Eur J Clin Nutr 49, 420-429 (1995).

28 Maasland, D. H., van den Brandt, P. A., Kremer, B., Goldbohm, R. A. \& Schouten, L. J. Alcohol consumption, cigarette smoking and the risk of subtypes of head-neck cancer: results from the Netherlands Cohort Study. BMC Cancer 14, 187 (2014).

29 Leffondre, K., Abrahamowicz, M., Siemiatycki, J. \& Rachet, B. Modeling smoking history: a comparison of different approaches. Am J Epidemiol 156, 813-823 (2002).

30 Negri, E. et al. Family history of cancer: pooled analysis in the International Head and Neck Cancer Epidemiology Consortium. Int. J. Cancer 124, 394-401 (2009).

31 Schoenfeld, D. Partial residuals for the proportional hazards regression model. Biometrika 69, 239-241 (1982).

32 Zheng, W. et al. Serum micronutrients and the subsequent risk of oral and pharyngeal cancer. Cancer Res 53, 795-798 (1993).

$33 \mathrm{Li}, \mathrm{Q}$. et al. Vitamin or mineral supplement intake and the risk of head and neck cancer: pooled analysis in the INHANCE consortium. Int J Cancer 131, 1686-1699 (2012).

34 Bardia, A. et al. Efficacy of antioxidant supplementation in reducing primary cancer incidence and mortality: systematic review and meta-analysis. Mayo Clin. Proc. 83, 23-34 (2008).

35 Bravi, F. et al. Foods, nutrients and the risk of oral and pharyngeal cancer. Br. J. Cancer 109, 2904-2910 (2013).

36 Marshall, J. R. et al. Smoking, alcohol, dentition and diet in the epidemiology of oral cancer. Eur. J. Cancer. B. Oral Oncol. 28B, 9-15 (1992).

37 Negri, E. et al. Selected micronutrients and oral and pharyngeal cancer. Int. J. Cancer 86, 122-127 (2000).

38 Petridou, E. et al. The role of diet and specific micronutrients in the etiology of oral carcinoma. Cancer 94, 2981-2988 (2002).

39 Lee, K. W., Lee, H. J., Surh, Y. J. \& Lee, C. Y. Vitamin C and cancer chemoprevention: reappraisal. Am. J. Clin. Nutr. 78, 1074-1078 (2003).

40 Mamede, A. C. et al. The role of vitamins in cancer: a review. Nutr. Cancer 63, 479-494 (2011).

41 Fiedor, J. \& Burda, K. Potential role of carotenoids as antioxidants in human health and disease. Nutrients 6, 466-488 (2014).

42 Cvetkovic, D., Fiedor, L., Fiedor, J., Wisniewska-Becher, A. \& Markovic, D. in Carotenoids: Food Sources, Production and Health Benefits (ed M. Yamaguchi) 93-126 Nova Science Publishers, 2013.

43 Edge, R. \& Truscott, G. in Carotenoids: physical, chemical, and biological functions and properties (ed J.T. Landrum), 283-308. CRC Press, 2010.

44 Fiedor, J., Fiedor, L., Haessner, R. \& Scheer, H. Cyclic endoperoxides of beta-carotene, potential pro-oxidants, as products of chemical quenching of singlet oxygen. Biochim. Biophys. Acta 1709, 1-4 (2005).

45 Bonassi, S. et al. Chromosomal aberrations in lymphocytes predict human cancer independently of exposure to carcinogens. European Study Group on Cytogenetic Biomarkers and Health. Cancer Res. 60, 1619-1625 (2000).

46 Lotan, R. Retinoids and apoptosis: implications for cancer chemoprevention and therapy. J. Natl. Cancer Inst. 87, 16551657 (1995). 
Supplemental Table 4.1. Sensitivity analyses (first two years of follow-up excluded): multivariable-adjusted $^{\text {a }}$ associations between vitamin and carotenoid intake and risk of head-neck cancer (HNC) overall; Netherlands Cohort Study, 1986-2006

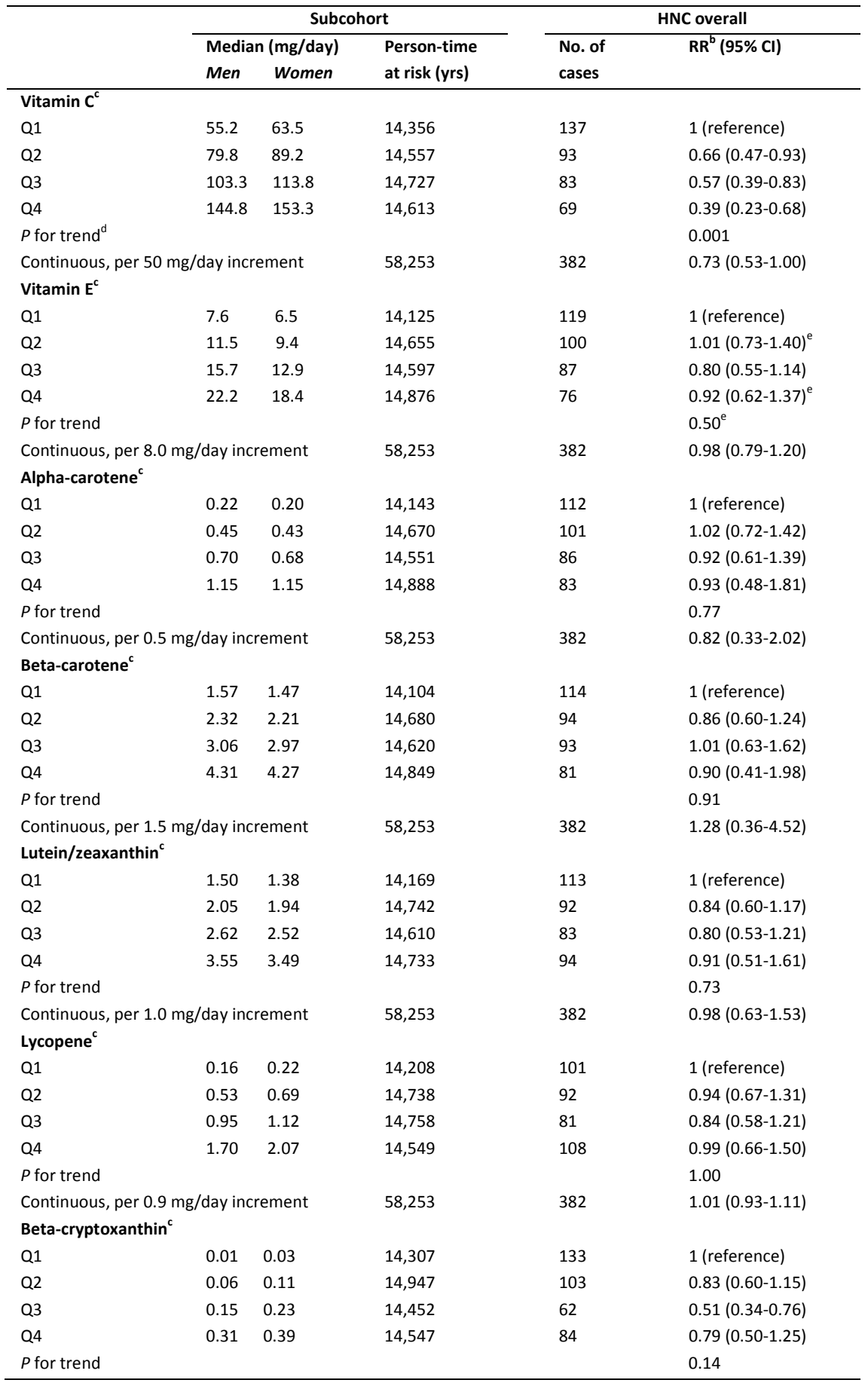




\begin{tabular}{|c|c|c|c|c|c|}
\hline \multirow[t]{3}{*}{ (continued) } & \multicolumn{3}{|c|}{ Subcohort } & \multicolumn{2}{|c|}{ HNC overall } \\
\hline & \multicolumn{2}{|c|}{ Median (mg/day) } & \multirow{2}{*}{$\begin{array}{l}\text { Person-time } \\
\text { at risk (yrs) }\end{array}$} & \multirow{2}{*}{$\begin{array}{l}\text { No. of } \\
\text { cases }\end{array}$} & \multirow[t]{2}{*}{ RR (95\% Cl) } \\
\hline & Men & Women & & & \\
\hline Continuous, $\mathrm{p}$ & /day in & ement & 58,253 & 382 & $1.15(0.86-1.55)$ \\
\hline \multicolumn{6}{|c|}{ Supplement vitamin C } \\
\hline No & & & 52,020 & 341 & 1 (reference) \\
\hline Yes & & & 6,233 & 41 & $1.21(0.76-1.92)$ \\
\hline \multicolumn{6}{|c|}{ Supplement vitamin E } \\
\hline No & & & 54,457 & 361 & 1 (reference) \\
\hline Yes & & & 3,796 & 21 & $0.93(0.51-1.69)$ \\
\hline
\end{tabular}

${ }^{a}$ Mutually adjusted for age (years); sex; cigarette smoking (status (never/former/current), frequency (number of cigarettes per day; continuous, centered), duration (number of years; continuous, centered)); alcohol consumption (grams ethanol per day; continuous); and total daily energy intake (kcal/day; continuous).

${ }^{\mathrm{b}}$ Abbreviations: RR: incidence rate ratio; $\mathrm{Cl}$ : confidence interval; Q: quartile.

${ }^{\mathrm{c}}$ Nutrient from food intake only.

${ }^{\mathrm{d}}$ Tests for dose-response trends were assessed by fitting ordinal exposure variables as continuous terms in the Cox proportional hazards model.

${ }^{\mathrm{e}}$ The proportional hazards assumption was possibly violated for the exposure variable in this analysis; there was no statistically significant interaction between the exposure variable and time. 
Chapter 5

Toenail selenium status and risk of subtypes of head-neck cancer: the Netherlands Cohort

Study

Denise H. E. Maasland

Leo J. Schouten

Bernd Kremer

Piet A. van den Brandt

Eur J Cancer 2016;60:83-92 


\begin{abstract}

\section{Background}

There is limited prospective data on the relationship between selenium status and the risk of head-neck cancer (HNC) and HNC subtypes (i.e., oral cavity cancer (OCC), oro/hypopharyngeal cancer (OHPC), and laryngeal cancer (LC)). Therefore, we investigated the association between toenail selenium, reflecting long-term selenium exposure, and HNC risk within the Netherlands Cohort Study.
\end{abstract}

\title{
Methods
}

At baseline, 120,852 participants completed a self-administered questionnaire about diet and other cancer risk factors and were asked to provide toenail clippings. After 20.3 years of follow-up, 294 cases of HNC (95 OCC, 62 OHPC, two oral cavity/pharynx unspecified or overlapping, and $135 \mathrm{LC}$ ) and 2,164 subcohort members were available for case-cohort analysis using Cox proportional hazards models.

\section{Results}

Toenail selenium status was statistically significantly associated with a decreased risk of HNC overall (multivariable-adjusted incidence rate ratio for quartile four vs. one: $0.55,95 \%$ confidence interval $0.37-0.82, P$ trend=0.001). The association between toenail selenium and risk of HNC overall was stronger among men than women, but no statistically significant interaction with sex was found. Toenail selenium level was also associated with a decreased risk of all HNC subtypes, with statistically significant associations in OHPC and LC. No statistically significant interaction was found between toenail selenium level and cigarette smoking or alcohol consumption for HNC overall.

\section{Conclusions}

In this large cohort study, we found an inverse association between toenail selenium level and HNC risk. Among HNC subtypes, this association was strongest for OHPC and LC. Furthermore, the association of toenail selenium status with HNC risk was stronger among men than women. 


\section{Introduction}

Selenium is a trace element present in food which is likely to be involved in cancer risk. It might act in various stages of tumor development and in several anticarcinogenic processes. ${ }^{1,2}$ Selenium functions as an essential component of selenoproteins, antioxidant enzymes-including glutathione peroxidases-that protect against oxidative damage. When selenium is incorporated into these selenoproteins, they may influence carcinogenesis by their antioxidant properties. ${ }^{1,2}$ Other mechanisms regarding the anticancer effect of selenium include alteration of DNA methylation, cell cycle regulation, enhancement of immune function, and inhibition of angiogenesis. ${ }^{1,2}$

Evidence for an effect of selenium on cancer risk has been reviewed and includes findings from different types of study, including in vitro, animal, and ecological studies. ${ }^{1}$ In observational studies, selenium status has been associated with a decreased risk of prostate and esophageal cancer and possibly also of lung and stomach cancer. ${ }^{3-5}$

A low selenium status may also be associated with an increased risk of head and neck cancer (HNC). HNC is the seventh most common type of cancer in the world and alcohol consumption, cigarette smoking, and human papillomavirus (HPV) infection are established risk factors for HNC., ${ }^{5,6}$ Evidence regarding selenium and HNC risk is, however, scarce.

In a population-based case-control study in the United States, low toenail selenium status was associated with an increased risk of oral cancer, but only in men. ${ }^{7}$ Two nested case-control studies in Finland and the United States yielded mixed results with respect to the association of serum selenium status and the risk of $\mathrm{HNC}$, but the number of cases in both studies was very small. ${ }^{8,9}$

Selenium status can be measured in various ways. Worldwide, the dietary intake of selenium varies considerably, with a relatively high intake in the United States compared to Europe, as a result of differences in selenium soil content. ${ }^{2}$ Consequently, selenium levels in food vary such that the measurement of selenium intake through food questionnaires is flawed. Blood selenium reflects selenium exposure over recent weeks, whereas toenail selenium reflects the intake of selenium for a period up to one year. ${ }^{10}$ Furthermore, given the leading role of smoking and alcohol consumption in the development of HNC, adequate adjustment for confounding by these factors is of great importance when investigating selenium and HNC risk.

We investigated the association between toenail selenium levels and total HNC and HNC subtypes within the large prospective Netherlands Cohort Study (NLCS). We focused on the most frequent HNC subtypes ${ }^{11}$ (those located in the oral cavity, 
pharynx, and larynx) and hypothesized that the risk of HNC is higher in participants with low levels of toenail selenium. In addition, we examined possible effect modification of the association between toenail selenium and HNC risk by sex, cigarette smoking, or alcohol consumption.

\section{Methods}

\section{Study design and population}

The present study was conducted within the NLCS, which was initiated in September 1986 and includes 120,852 participants, aged 55-69 years from 204 Dutch municipal population registries. ${ }^{12}$ At baseline, all participants completed a self-administered questionnaire about diet, lifestyle habits, and other cancer risk factors. In addition, they were asked to provide toenail clippings; approximately $75 \%$ of the subjects provided these. The NLCS has been approved by the Medical Ethics Committee of Maastricht University (Maastricht, The Netherlands). Participants were informed that by returning the questionnaire and toenail sample they would give their consent to participate in a study of the etiology of cancer.

We used the case-cohort design for efficiency in data processing and follow-up. ${ }^{13}$ Cases were derived from the entire cohort, whereas the number of person-years at risk for the entire cohort was estimated from a subcohort of 3,500 people who were randomly sampled from the total cohort at baseline.

Follow-up for cancer incidence was done by record linkage to the Netherlands Cancer Registry (NCR) and the nationwide network and pathology registry (PALGA). ${ }^{14}$ Follow-up for vital status of the subcohort was nearly $100 \%$ complete after 20.3 years and the completeness of cancer follow-up is estimated to be $\geq 96 \%{ }^{15}$

Only microscopically confirmed, first occurrences of squamous cell carcinomaswhich include nearly all malignancies of the mouth, pharynx, and larynx-were included in our study (Figure 5.1). ${ }^{5,16}$ We excluded cohort members who reported having prevalent cancer other than skin cancer at baseline. In addition, we excluded participants who had incomplete/inconsistent dietary data, did not provide a toenail sample, had a sample with a too low sample weight $(<10 \mathrm{mg})$, or had missing data on confounding variables (see below). ${ }^{17}$

Complete data for statistical analysis with toenail selenium were available for 2,164 subcohort members and 294 incident cases of the selected HNC subtypes (Figure 5.1). HNC subtypes were classified as proposed by Hashibe et al. ${ }^{18}$ according to the International Classification of Diseases for Oncology ${ }^{19}$ (Table 5.1). 
Table 5.1. Subclassification of subtypes of head-neck cancer (HNC) as proposed by Hashibe et al. ${ }^{18}$, according to the International Classification of Diseases for Oncology, version 3 (ICD-O-3). ${ }^{19}$

\begin{tabular}{|c|c|}
\hline HNC-subtype & ICD-0-3 \\
\hline \multirow[t]{2}{*}{ Oral cavity cancer (OCC) } & C003-009, C020-C023, C030-C031, C039-C041, C048- \\
\hline & $\mathrm{C} 050, \mathrm{C} 060-\mathrm{C} 062, \mathrm{C068-C069}$ \\
\hline \multirow[t]{2}{*}{ Oro-/hypopharyngeal cancer (OHPC) } & C019, C024, C051-C052, C090-C091, C098-C104, C108- \\
\hline & C109, C129-C132, C138-C139 \\
\hline Oral cavity, pharynx unspecified or overlapping cancer (USC) & C028-C029, C058-C059, C140-C142, C148 \\
\hline Laryngeal cancer (LC) & C320-C329 \\
\hline
\end{tabular}

Of the 294 HNC cases, 95 were oral cavity cancer (OCC), 62 were oro-/hypopharyngeal cancer (OHPC), two were oral cavity/pharynx unspecified or overlapping (only included in analyses of HNC overall), and 135 were laryngeal cancer (LC) cases.

\begin{tabular}{|c|c|c|c|c|}
\hline \multicolumn{5}{|c|}{ Netherlands Cohort Study on diet and cancer $(120,852)$} \\
\hline$\downarrow$ & & & $\downarrow$ & \\
\hline $\begin{array}{l}\text { Subcohort, randomly } \\
\text { sampled from total cohort }\end{array}$ & \multicolumn{4}{|c|}{ Record linkage with Netherlands Cancer Registry and PALGA } \\
\hline $\begin{array}{l}\downarrow \\
3,500\end{array}$ & $\begin{array}{l}\downarrow \\
\text { HNC overall }^{a}\end{array}$ & $\begin{array}{l}\qquad \\
\qquad \\
\text { Oral cavity } \\
\text { cancer (OCC) }\end{array}$ & $\begin{array}{l}\qquad \\
\qquad \\
\text { Oro-/hypopharyngeal } \\
\text { cancer (OHPC) }\end{array}$ & $\begin{array}{l}\downarrow \\
\text { Laryngeal } \\
\text { cancer (LC) }\end{array}$ \\
\hline \multicolumn{5}{|c|}{ Exclusion of participants with prevalent cancer at baseline } \\
\hline $\begin{array}{c}\downarrow \\
3,346\end{array}$ & $\begin{array}{c}\downarrow \\
515\end{array}$ & $\begin{array}{c}\downarrow \\
149\end{array}$ & $\begin{array}{c}\downarrow \\
109\end{array}$ & $\begin{array}{c}\downarrow \\
254\end{array}$ \\
\hline \multicolumn{5}{|c|}{ Exclusion of participants with incomplete or inconsistent dietary data } \\
\hline $\begin{array}{c}\downarrow \\
3,128\end{array}$ & $\underset{473}{\downarrow}$ & $\begin{array}{c}\downarrow \\
140\end{array}$ & $\begin{array}{c}\downarrow \\
102\end{array}$ & $\begin{array}{c}\downarrow \\
228\end{array}$ \\
\hline \multicolumn{5}{|c|}{ Exclusion of participants with missing data on selenium } \\
\hline $\begin{array}{c}\downarrow \\
2,328\end{array}$ & $\begin{array}{c}\downarrow \\
323\end{array}$ & $\begin{array}{c}\downarrow \\
100\end{array}$ & $\begin{array}{l}\downarrow \\
71\end{array}$ & $\begin{array}{c}\downarrow \\
150\end{array}$ \\
\hline \multicolumn{5}{|c|}{ Exclusion of participants with missing data on confounders } \\
\hline $\begin{array}{c}\downarrow \\
2,164\end{array}$ & $\begin{array}{c}\downarrow \\
294\end{array}$ & $\begin{array}{l}\downarrow \\
95\end{array}$ & $\begin{array}{l}\downarrow \\
62\end{array}$ & $\begin{array}{c}\downarrow \\
135\end{array}$ \\
\hline
\end{tabular}

Figure 5.1. Flow diagram of the number of subcohort members and cases on whom the analyses were based. Abbreviation PALGA: nationwide network and registry of histopathology and cytopathology in the Netherlands.

${ }^{a}$ Oral cavity cancer; oro-/hypopharyngeal cancer; oral cavity, pharynx unspecified or overlapping cancer; laryngeal cancer.

\section{Exposure data}

Selenium concentrations were measured in toenail clippings by the Reactor Institute Delft (Delft University of Technology; Delft, The Netherlands), using instrumental neutron activation analysis of the ${ }^{77 m}$ Se isotope (metastable selenium-77 isotope, halflife 17.5 seconds). Each sample went through six cycles of 17 -second irradiation at a thermal neutron flux of $3 * 10^{16} \mathrm{~m}^{-2} \mathrm{~s}^{-1}, 3$-second decay, and 17 -second counting at $1 \mathrm{~cm}$ 
from a $40 \%$ germanium detector. The accuracy of the neutron activation analysis was checked by analysis of a certified bovine liver standard (Standard Reference Material 1577b, later replaced by 1577c, of the United States National Institute of Standards and Technology). This method and the NLCS toenail selenium project have been described in more detail previously. ${ }^{4,20,21}$

The toenail selenium measurements for the subcohort were carried out in 1992. In 2012-2013, toenail selenium levels of HNC cases diagnosed during 20.3 years of followup were measured. In 1992, the 'Snelle Buizen Post' (SBP) facility was used for instrumental neutron activation analysis, and, since 1996, the 'Carbonfiber Autonomous Facility for Irradiation and Analysis' (CAFIA) facility is used. To assess the validity and comparability of these two methods, toenail selenium levels of the same 40 subcohort members were determined in 1996 with the 'CAFIA' facility in addition to the original assessment with the 'SBP' facility. ${ }^{22}$ The mean selenium level ( $\mu \mathrm{g} / \mathrm{g}$ ) assessed by the 'CAFIA' facility (0.552; standard deviation (SD): 0.05 ) was similar to the mean selenium level $(\mu \mathrm{g} / \mathrm{g}$ ) assessed by the 'SBP' facility $(0.551 ; \mathrm{SD}: 0.04)$, with a Pearson correlation coefficient of $0.95(P<0.01) .{ }^{22}$ It was concluded that both methods were valid and comparable.

The questionnaire included a 150 -item food frequency questionnaire that focused on habitual food consumption during the year preceding the start of the study. In addition, we asked questions about lifestyle habits and other cancer risk factors. We asked detailed questions about alcohol consumption and cigarette smoking, as described before. ${ }^{23}$ Data were key-entered and processed in a standardized manner, blinded with respect to case/subcohort status in order to minimize observer bias in coding and data interpretation.

\section{Statistical analysis}

The Cox proportional hazards model was used to estimate age- and sex-adjusted and multivariable-adjusted incidence rate ratios (RR) and corresponding 95\% confidence intervals $(\mathrm{Cl})$. Person-years at risk were calculated from baseline until diagnosis of HNC, death, emigration, loss to follow-up, or end of follow-up, whichever occurred first. For analyses of HNC overall, we categorized toenail selenium levels into quartiles according to the sex-specific distribution in the subcohort. For analyses of HNC subtypes, we decided to categorize toenail selenium levels into sex-specific tertiles because of the limited number of cases. For continuous analyses, selenium levels were standardized to the average size of the two central quartiles in the subcohort; one standardized selenium unit equals $0.08 \mu \mathrm{g} / \mathrm{g}$. 
To evaluate the potential influence of prediagnostic cancer at baseline on toenail selenium levels, cases were categorized according to the year of follow-up in which they were diagnosed. We compared the mean selenium level of HNC cases diagnosed during the first two years of follow-up with the level of cases diagnosed later in followup. In addition, after we applied a natural logarithm (In)-transformation to normalize the distribution of selenium levels, we used an independent samples t-test to test the statistical significance of differences. Based on these results, we decided to include the total follow-up time in our analyses. We also performed sensitivity analyses regarding the association between toenail selenium status and the risk of HNC overall by excluding the first two years of follow-up.

The predefined confounders were age (years); sex; alcohol consumption (grams/day); and cigarette smoking (status (never/former/current), number of cigarettes smoked daily, and number of smoking years). We considered the following potential confounders: level of education; body mass index; non-occupational physical activity; intake of vegetables and fruits, fish, red meat, and meat products (all grams/day); and family history of HNC., ${ }^{5,24}$ None of these variables changed the RR for toenail selenium (continuous) for HNC overall or any of the HNC subtypes by $>10 \%$ when including them in the model. Therefore, the final model included only the predefined confounders. When adjusting for cigarette smoking frequency and duration, we centered these continuous variables as proposed by Leffondré et al. ${ }^{25}$

We assessed tests for linear dose-response trends by fitting ordinal exposure variables as continuous terms. Standard errors were estimated using the robust HuberWhite sandwich estimator to account for additional variance due to sampling from the cohort. The proportional hazards (PH) assumption was assessed using the scaled Schoenfeld residuals. ${ }^{26}$ If there was an indication for violation of the $\mathrm{PH}$ assumption for a variable, it was further investigated by adding a time-varying covariate for that variable to the model. We performed analyses for HNC and all HNC subtypes using a time-varying covariate for current smoking, as described before. ${ }^{27}$

To determine whether sex, cigarette smoking, or alcohol consumption possibly modify the association of toenail selenium status (both on a categorical and continuous scale) with risk of HNC overall, we estimated RRs in strata of these exposures. Tests for interaction were performed with toenail selenium levels on a categorical (quartiles) and continuous scale. $P$ values for interaction were assessed by including cross-product terms in the models and performing a Wald test.

No statistically significant interaction with sex was found. However, we noticed considerable differences in risk estimates for HNC overall between men and women. Therefore (and because of relatively few female cases in HNC subtypes), analyses were 
carried out for both sexes combined and, for HNC overall, also for men and women separately. We performed analyses in strata of alcohol consumption (abstainers; consuming $>0$ to 15 grams ethanol/day; consuming $\geq 15$ grams ethanol/day) and cigarette smoking status (never/former/current). Alcohol consumption and cigarette smoking were mutually adjusted in these models.

All reported $P$ values were based on two-sided tests and considered statistically significant if $<0.05$. Analyses were done using the Stata 11.2 statistical software package (StataCorp, College Station, Texas, USA).

\section{Results}

\section{Baseline characteristics}

Mean toenail selenium levels were considerably lower among cases $(0.517 \mu \mathrm{g} / \mathrm{g})$ than subcohort members $(0.562 \mu \mathrm{g} / \mathrm{g})$ (Table 5.2$)$. Compared with subcohort members, cases were far more often men than women. Furthermore, among subcohort members, men had noticeably lower toenail selenium levels $(0.549 \mu \mathrm{g} / \mathrm{g})$ than women $(0.575 \mu \mathrm{g} / \mathrm{g})$. There were also far more current smokers among cases than subcohort members, and the frequency and duration of cigarette smoking were higher among cases. Finally, cases were less frequently alcohol abstainers and had a substantially higher alcohol intake than subcohort members.

There was no clear trend toward higher or lower toenail selenium levels in the HNC cases in the course of 20.3 years of follow-up (Table 5.3). HNC overall cases diagnosed after the second year of follow-up ( $N=269)$ had a mean toenail selenium level of $0.519 \mu \mathrm{g} / \mathrm{g}$; cases diagnosed during the first two years of follow-up ( $N=25)$ had a mean toenail selenium level of $0.494 \mu \mathrm{g} / \mathrm{g}$. This difference was not statistically significant.

\section{Main analyses}

Results from age- and sex-adjusted and multivariable-adjusted analyses both showed mostly inverse associations between toenail selenium level and HNC risk, but most associations were substantially stronger in age- and sex-adjusted analyses, especially in HNC subtypes (Tables 5.4 and 5.5).

Toenail selenium status was statistically significantly associated with a decreased risk of HNC overall, on a categorical (multivariable-adjusted RR for quartile four vs. one: $0.55,95 \% \mathrm{Cl}$ 0.37-0.82, $\mathrm{P}$ trend=0.001) and continuous scale (multivariableadjusted RR per increment in standard selenium unit: $0.82,95 \% \mathrm{Cl} 0.73-0.91$ ) (Table 
5.4). The association between toenail selenium and risk of HNC overall was considerably stronger among men (RR: $0.46,95 \% \mathrm{Cl} 0.28-0.76$ for quartile four vs. one, $P$ trend<0.001) than women (RR: $0.89,95 \% \mathrm{Cl} 0.44-1.83, \mathrm{P}$ trend $=0.63$ ), but no statistically significant interaction with sex was found ( $P=0.28$ (continuous scale) and 0.43 (categorical)). Sensitivity analyses showed essentially similar results after exclusion of the first two years of follow-up.

Among HNC subtypes, toenail selenium level was associated with a decreased risk of HNC, with statistically significant associations in OHPC (multivariable-adjusted RR per increment in standard selenium unit: $0.72,95 \% \mathrm{Cl} 0.58-0.90)$ and LC (RR per standard unit: $0.83,95 \% \mathrm{Cl} 0.71-0.96$ ) but not OCC (RR per standard unit: $0.88,95 \% \mathrm{Cl}$ $0.75-1.04$ ) (Table 5.5). For all subtypes, decreased risks were also found across tertiles of toenail selenium, but without statistical significance. The inverse associations between toenail selenium and HNC risk were generally strongest for OHPC.

\section{Stratified analyses}

No statistically significant interaction was found between quartiles of toenail selenium level and either cigarette smoking $(P$ interaction $=0.94)$ or alcohol consumption $(P$ interaction=0.32) for HNC overall (Table 5.6). Within strata of cigarette smoking, we found largely inverse associations between toenail selenium and HNC; we found a statistically significantly decreased risk of HNC overall for current smokers in the highest compared with the lowest quartile. Stratified analyses with toenail selenium on a continuous scale showed the same pattern. Within strata of alcohol consumption, we found mostly inverse associations between toenail selenium and HNC risk in all strata as well (Table 5.6). 


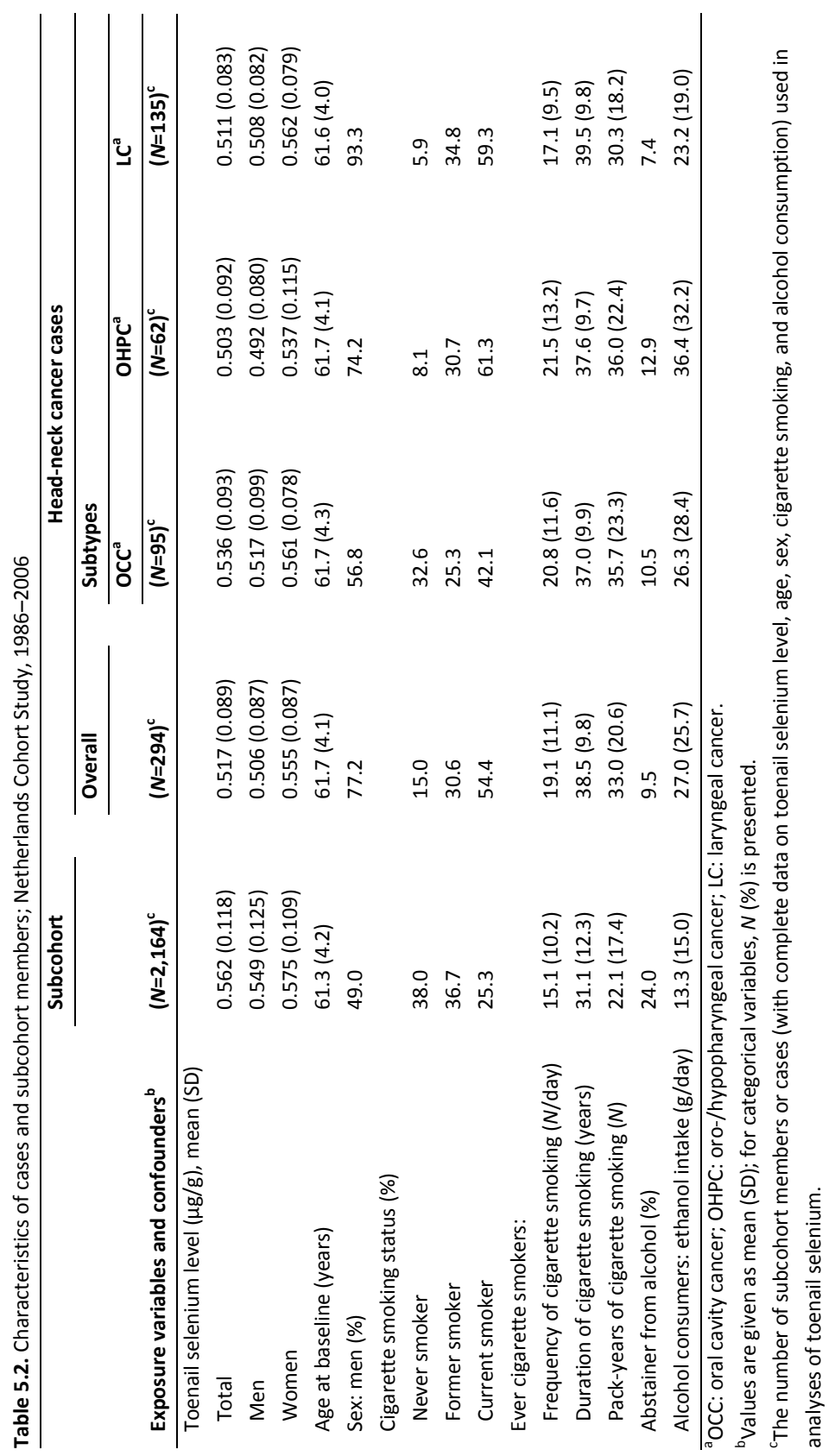


Table 5.3. Toenail selenium levels in head-neck cancer (HNC) cases according to sex and time between baseline and HNC diagnosis; Netherlands Cohort Study, 1986-2006

\begin{tabular}{|c|c|c|c|c|}
\hline \multirow[b]{3}{*}{ Cases $^{a}$} & \multicolumn{4}{|c|}{ Head-neck cancer overall } \\
\hline & \multirow[b]{2}{*}{ No. of cases } & \multicolumn{3}{|c|}{ Toenail selenium level $(\mu \mathrm{g} / \mathrm{g})$} \\
\hline & & Mean & SD & $P$ value \\
\hline All cases & 294 & 0.517 & 0.089 & \\
\hline Men & 227 & 0.506 & 0.087 & \\
\hline Women & 67 & 0.555 & 0.087 & \\
\hline \multicolumn{5}{|c|}{ Year of follow-up } \\
\hline $0-2$ & 25 & 0.494 & 0.098 & \\
\hline$>2$ & 269 & 0.519 & 0.088 & $0.13^{b}$ \\
\hline \multicolumn{5}{|c|}{ Categories year of follow-up } \\
\hline $0-2$ & 25 & 0.494 & 0.098 & \\
\hline$>2-4$ & 31 & 0.520 & 0.087 & \\
\hline$>4-6$ & 32 & 0.497 & 0.087 & \\
\hline$>6-8$ & 31 & 0.508 & 0.072 & \\
\hline$>8-10$ & 31 & 0.514 & 0.090 & \\
\hline$>10-12$ & 33 & 0.532 & 0.110 & \\
\hline$>12-14$ & 23 & 0.487 & 0.084 & \\
\hline$>14-16$ & 37 & 0.522 & 0.085 & \\
\hline$>16-18$ & 30 & 0.559 & 0.076 & \\
\hline$>18$ & 21 & 0.530 & 0.087 & \\
\hline
\end{tabular}

${ }^{a}$ Mean \pm standard deviation (SD) selenium levels in subcohort members were $0.549 \pm 0.125 \mu \mathrm{g} / \mathrm{g}$ for men $(N=1,061)$ and $0.575 \pm 0.109 \mu \mathrm{g} / \mathrm{g}$ for women $(N=1,103)$.

${ }^{\mathrm{b}} T$-test of mean toenail selenium level (natural logarithm [In]-transformed) in first two years of follow-up vs. rest of follow-up years. 


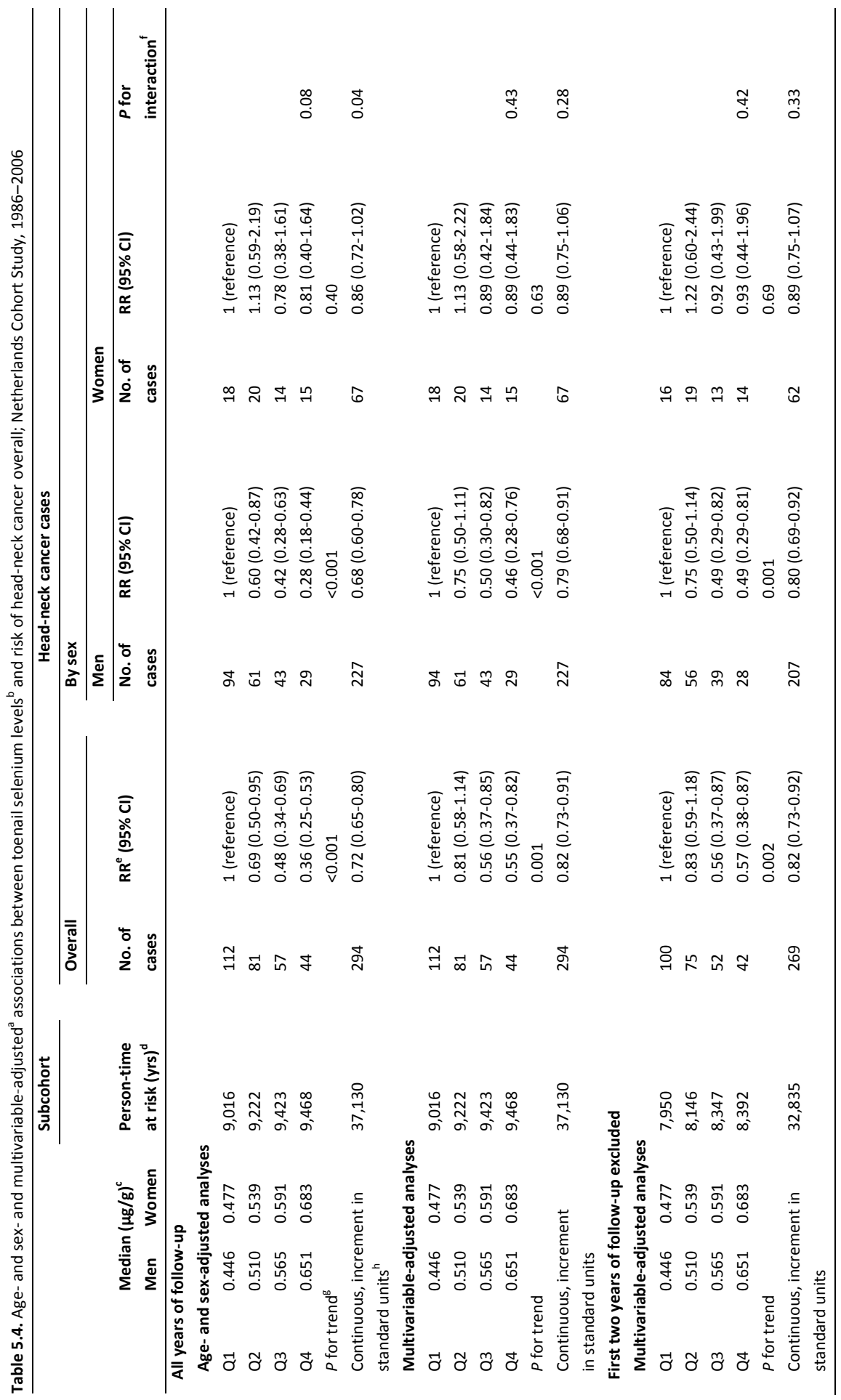


Table 5.4 (footnotes).

${ }^{a}$ Adjusted for age (years); sex; cigarette smoking (status (never/former/current), frequency (number of cigarettes per day; continuous, centered), duration (number of years; continuous, centered)); and alcohol consumption (grams ethanol per day; continuous).

${ }^{\mathrm{b}}$ Quartiles of toenail selenium $(\mu \mathrm{g} / \mathrm{g})$.

'Quartile boundaries ( $\mu \mathrm{g} / \mathrm{g}$ ) men: Q1: 0.183-0.483; Q2: 0.483-0.539; Q3: 0.539-0.601; Q4: 0.602-2.605; women: Q1: 0.1090.513; Q2: 0.513-0.563; Q3: 0.563-0.622; Q4: 0.622-1.329.

${ }^{\mathrm{d}}$ The total person-time at risk was 17,135 person-years for men and 19,995 person-years for women; in sensitivity analyses, men and women had 15,036 and 17,799 person-years at risk, respectively.

${ }^{\mathrm{e}}$ Abbreviations: RR: incidence rate ratio; $\mathrm{Cl}$ : confidence interval; Q: quartile.

${ }^{f} P$ value for interaction between sex and toenail selenium level (categorical and continuous), based on cross-product terms in the Cox proportional hazards model and Wald test.

${ }^{\mathrm{g}}$ Tests for dose-response trends were assessed by fitting ordinal exposure variables as continuous terms in the Cox proportional hazards model.

${ }^{h}$ For continuous analyses, selenium levels were standardized to the average size of the two central quartiles; one standardized selenium unit equals $0.08 \mu \mathrm{g} / \mathrm{g}$. 


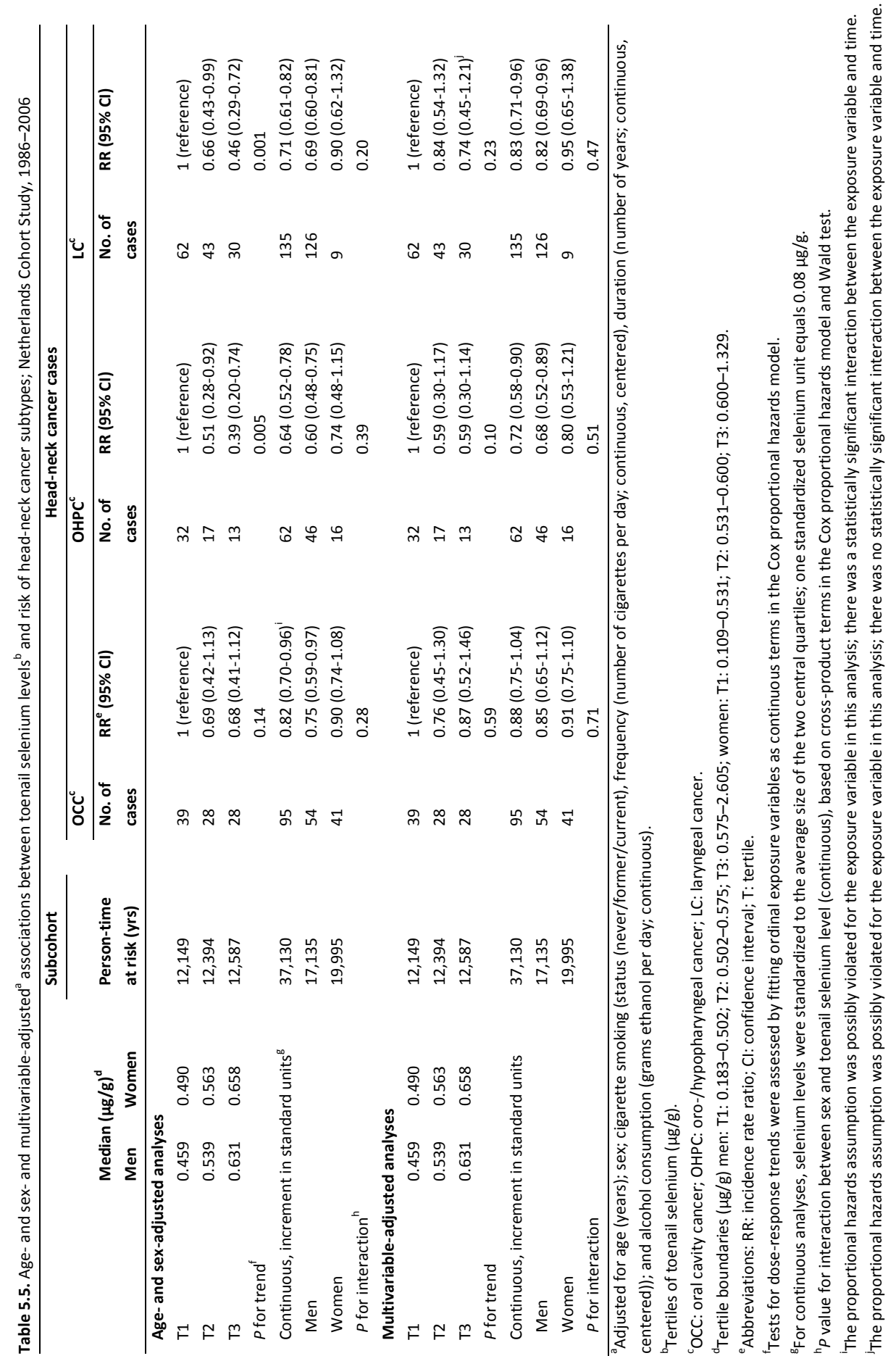




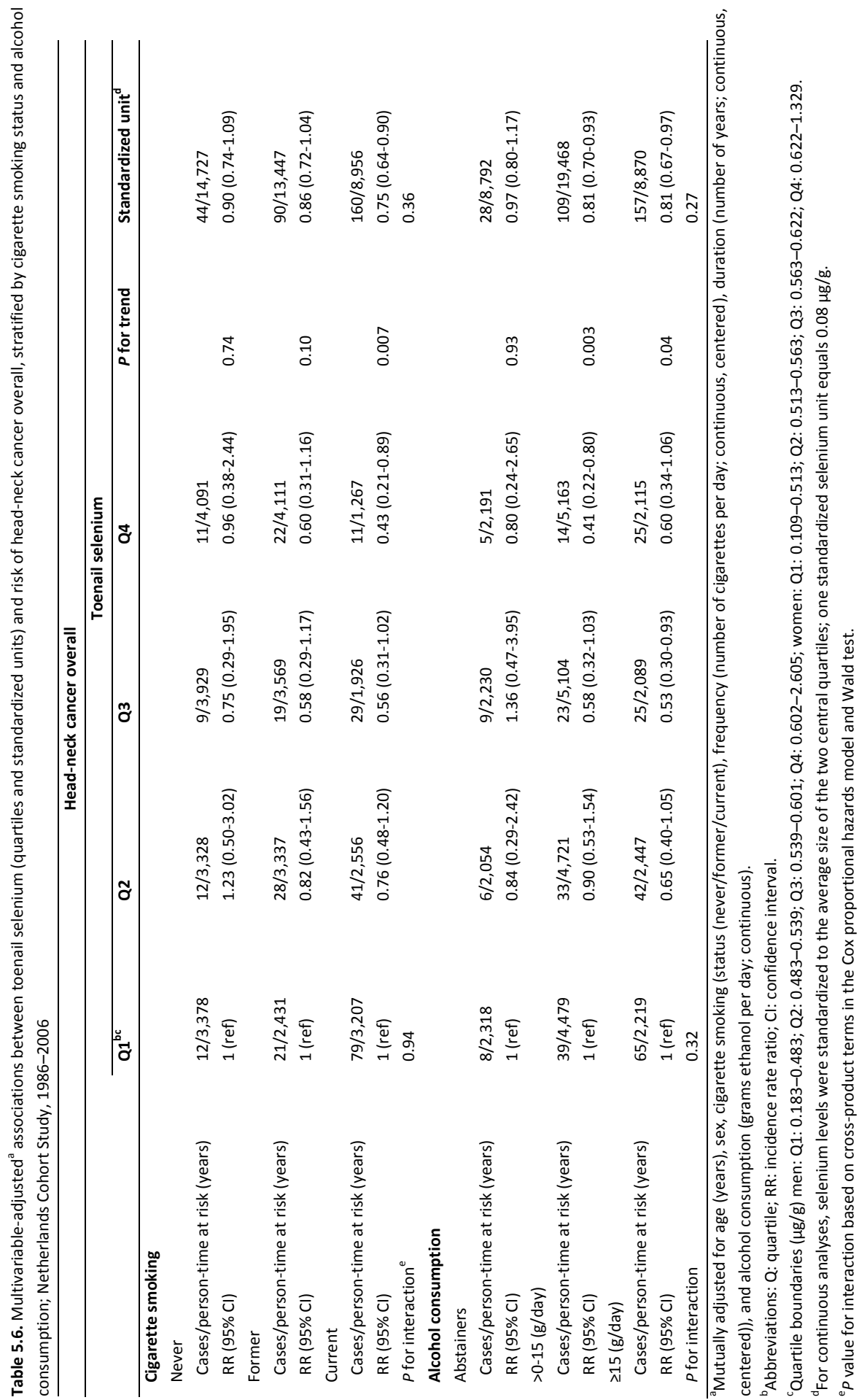




\section{Discussion}

In this large prospective cohort study, we found a strong inverse association between toenail selenium status and risk of HNC overall. The association between toenail selenium and risk of HNC overall was considerably stronger among men than women, with a non-significant association in women. Among HNC subtypes, toenail selenium status showed the strongest inverse association with OHPC and LC. Although there was no clear effect modification by cigarette smoking or alcohol consumption in our study, the association we found was most explicit among current smokers. Mechanisms of action of the possible HNC risk reducing effect of selenium probably involve the antioxidant and other anticarcinogenic properties of selenium, including cell cycle regulation. ${ }^{1,2}$

\section{Toenail selenium and risk of HNC and HNC subtypes}

Previous observational studies showed mixed results regarding selenium and HNC risk, but most had small numbers. In a population-based case-control study with 379 oral cancer cases in the United States, low toenail selenium status was associated with an increased risk of oral cancer ${ }^{7}$ as well, with an odds ratio for low selenium levels in nail tissue (lowest quartile compared with the highest quartile) of 1.4 (95\% Cl 1.0-2.2). In addition, an inverse association between selenium levels and oral cancer was found in men, but not in women. In a nested case-control study with 28 cases of oral and pharyngeal cancer in the United States ${ }^{9}$, smoking-adjusted relative odds for oral cancer were $1.00,4.26$, and 5.43 with increasing tertile levels of serum selenium, in contrast to the inverse association we found. This finding, opposite to ours, may be the result of the very small number of cases in this study by Zheng et al. ${ }^{9}$ Furthermore, a nestedcase control study in Finland with only $31 \mathrm{HNC}$ cases found a crude relative risk increase per one standard deviation of serum selenium of 1.60 ( $P$ trend $=0.19)$ for cancer of the lip, oral cavity, and pharynx, and 0.68 ( $P$ trend=0.39) for laryngeal cancer. ${ }^{8}$ A systematic literature review by the World Cancer Research Fund ${ }^{5}$ concluded that data regarding selenium and HNC risk were either of too low quality, too inconsistent, or the number of studies too few to allow conclusions to be drawn. Therefore, our large prospective cohort study adds significantly to the existing evidence regarding the association between selenium and HNC risk, strongly suggesting an inverse association.

The differential risk we found between toenail selenium and HNC in men and women was found previously, but in a study of oral cancer. ${ }^{7}$ The difference we observed does not seem to be the result of differential smoking behavior among men 
and women, as this difference remains after extensive adjustment for smoking. However, toenail selenium status was higher in women than men, which may have accounted for some of the difference, as well as the small number of female cases. It is unclear what other reasons there might be behind this possible difference.

\section{Possible interaction between alcohol, smoking, and toenail selenium}

In stratified analyses, we showed that toenail selenium was inversely associated with HNC risk especially in current smokers, with no statistically significant interaction. However, the analysis included only 44 cases among never smokers, and there may be a lack of power to detect a significant deviation from the multiplicative interaction model. In addition, among HNC subtypes, strongest associations were found for OHPC and LC, which we have previously shown to be the subtypes most associated with cigarette smoking. ${ }^{23}$ Both observations might point to the possibility that, despite elaborate adjustment for confounding by smoking, there might still be residual confounding. The fact that most associations were substantially stronger in age- and sex-adjusted analyses than in multivariable-adjusted analyses further implies this possibility. On the other hand, it may also indicate that the effect of selenium on HNC risk is associated with smoking, which might be biologically plausible since selenium is an antioxidant and counteracts the oxidative stress caused by smoking. ${ }^{28}$ With regard to alcohol consumption, we did not find a statistically significant interaction either, but we cannot rule out possible residual confounding or effect modification.

\section{Strengths and limitations}

Strengths of our study are the prospective nature, the completeness and duration of follow-up, and our large case-number. We also had the ability to study HNC subtypes and to adjust for confounders extensively. Finally, we were able to accurately measure selenium in toenails, which reflects long-term selenium intake, and to study a wide range of selenium, including low selenium levels. ${ }^{2}$

A possible limitation of our study is that we only measured selenium at baseline without repeated exposure measurements, which may have led to bias due to misclassification of exposure. In addition, residual confounding due to cigarette smoking and alcohol consumption might have introduced bias, as described above. Furthermore, we have no data on HPV infection. Finally, the higher selenium status in women may have limited the possibility of detecting a significant association in women.

Although it would surely be interesting from a clinical point of view, we believe more research is warranted before putting ours and other findings regarding selenium and $\mathrm{HNC}$ risk into lifestyle recommendations or any clinical advice. It would not be 
scientifically grounded to make a statement about the exact role of selenium in HNC risk or give any advice based on our results and other available evidence, and we believe we should be conservative doing so for several reasons. Most importantly, it is necessary to examine our results in other large prospective cohort studies first. In addition, numerous other (lifestyle) factors are involved in HNC risk as well, including cigarette smoking and alcohol consumption. Finally, we believe more clinically oriented studies are needed as well before possibly translating our findings into recommendations.

\section{Conclusions}

In conclusion, we found an inverse association between toenail selenium level and HNC risk in this large cohort study. Among HNC subtypes, this association was strongest for OHPC and LC. Furthermore, associations of toenail selenium status with HNC risk may be modified by gender, cigarette smoking, and alcohol consumption. Future studies are warranted for confirmation of the association between toenail selenium and HNC risk.

\section{References}

1 Rayman, M. P. Selenium in cancer prevention: a review of the evidence and mechanism of action. The Proceedings of the Nutrition Society 64, 527-542 (2005).

2 Rayman, M. P. Selenium and human health. Lancet 379, 1256-1268 (2012).

3 Geybels, M. S., Verhage, B. A., van Schooten, F. J., Goldbohm, R. A. \& van den Brandt, P. A. Advanced prostate cancer risk in relation to toenail selenium levels. J Nat/ Cancer Inst 105, 1394-1401 (2013).

4 Steevens, J., van den Brandt, P. A., Goldbohm, R. A. \& Schouten, L. J. Selenium status and the risk of esophageal and gastric cancer subtypes: the Netherlands cohort study. Gastroenterology 138, 1704-1713 (2010).

5 World Cancer Research Fund \& American Institute for Cancer Research. Food, Nutrition, Physical Activity and the Prevention of Cancer: a Global Perspective. American Institute for Cancer Research, 2007.

6 IARC. A Review of Human Carcinogens: Biological Agents. Vol. 100B International Agency for Research on Cancer, 2012.

7 Rogers, M. A. et al. A case-control study of oral cancer and pre-diagnostic concentrations of selenium and zinc in nail tissue. Int J Cancer 48, 182-188 (1991).

8 Knekt, P. et al. Serum micronutrients and risk of cancers of low incidence in Finland. Am J Epidemiol 134, 356-361 (1991).

9 Zheng, W. et al. Serum micronutrients and the subsequent risk of oral and pharyngeal cancer. Cancer Res 53, 795-798 (1993).

10 Longnecker, M. P. et al. Use of selenium concentration in whole blood, serum, toenails, or urine as a surrogate measure of selenium intake. Epidemiology 7, 384-390 (1996).

11 Dobrossy, L. Epidemiology of head and neck cancer: magnitude of the problem. Cancer Metastasis Rev 24, 9-17 (2005).

12 van den Brandt, P. A. et al. A large-scale prospective cohort study on diet and cancer in The Netherlands. J Clin Epidemiol 43, 285-295 (1990).

13 Barlow, W. E., Ichikawa, L., Rosner, D. \& Izumi, S. Analysis of case-cohort designs. J Clin Epidemiol 52, 1165-1172 (1999). 
14 van den Brandt, P. A., Schouten, L. J., Goldbohm, R. A., Dorant, E. \& Hunen, P. M. Development of a record linkage protocol for use in the Dutch Cancer Registry for Epidemiological Research. Int J Epidemiol 19, 553-558 (1990).

15 Goldbohm RA, Van den Brandt PA \& Dorant E. Estimation of the coverage of Dutch municipalities by cancer registries and PALGA based on hospital discharge data. Tijdschr Soc Gezondheidsz, 80-84 (1994).

16 Argiris, A., Karamouzis, M. V., Raben, D. \& Ferris, R. L. Head and neck cancer. Lancet 371, 1695-1709 (2008).

17 Goldbohm, R. A. et al. Validation of a dietary questionnaire used in a large-scale prospective cohort study on diet and cancer. Eur J Clin Nutr 48, 253-265 (1994).

18 Hashibe, M. et al. Alcohol drinking in never users of tobacco, cigarette smoking in never drinkers, and the risk of head and neck cancer: pooled analysis in the International Head and Neck Cancer Epidemiology Consortium. J Natl Cancer Inst 99, 777-789 (2007).

19 Fritz, A. G. International Classification of Diseases for Oncology: ICD-O. 3rd edn, World Health Organization, 2000.

20 van den Brandt, P. A. et al. A prospective cohort study on toenail selenium levels and risk of gastrointestinal cancer. Journal of the National Cancer Institute 85, 224-229 (1993).

21 van den Brandt, P. A. et al. Predictors of toenail selenium levels in men and women. Cancer Epidemiol Biomarkers Prev 2, 107-112 (1993).

22 Zeegers, M. P., Goldbohm, R. A., Bode, P. \& van den Brandt, P. A. Prediagnostic toenail selenium and risk of bladder cancer. Cancer Epidemiol Biomarkers Prev 11, 1292-1297 (2002).

23 Maasland, D. H., van den Brandt, P. A., Kremer, B., Goldbohm, R. A. \& Schouten, L. J. Alcohol consumption, cigarette smoking and the risk of subtypes of head-neck cancer: results from the Netherlands Cohort Study. BMC Cancer 14, 187 (2014).

24 Negri, E. et al. Family history of cancer: pooled analysis in the International Head and Neck Cancer Epidemiology Consortium. Int J Cancer 124, 394-401 (2009).

25 Leffondre, K., Abrahamowicz, M., Siemiatycki, J. \& Rachet, B. Modeling smoking history: a comparison of different approaches. Am J Epidemiol 156, 813-823 (2002).

26 Schoenfeld, D. Partial residuals for the proportional hazards regression model. Biometrika 69, 239-241 (1982).

27 Maasland, D. H., van den Brandt, P. A., Kremer, B., Goldbohm, R. A. \& Schouten, L. J. Consumption of vegetables and fruits and risk of subtypes of head-neck cancer in the Netherlands Cohort Study. Int J Cancer 136, E396-409 (2015).

28 Rahman, K. Studies on free radicals, antioxidants, and co-factors. Clinical interventions in aging 2, 219-236 (2007). 
Chapter 6

Body mass index and risk of subtypes of headneck cancer: the Netherlands Cohort Study

Denise H. E. Maasland Piet A. van den Brandt

Bernd Kremer Leo J. Schouten 


\section{Abstract}

\section{Background}

Low body mass index (BMI) has been associated with risk of head-neck cancer (HNC), but prospective data are scarce. We investigated the association between BMI, BMI at age 20 years, and change in BMI during adulthood with risk of HNC and HNC subtypes.

\section{Methods}

120,852 participants completed a questionnaire on diet and other cancer risk factors, including anthropometric measurements, at baseline in 1986. After 20.3 years of follow-up, 411 HNC (127 oral cavity cancer (OCC), 84 oro-/hypopharyngeal cancer (OHPC), and 197 laryngeal cancer (LC)) cases and 3,980 subcohort members were available for case-cohort analysis using Cox proportional hazards models.

\section{Results}

$\mathrm{BMI}$ at baseline was inversely associated with risk of HNC overall, with a multivariableadjusted incidence rate ratio of 3.31 (95\% $\mathrm{Cl} 1.40-7.82)$ for subjects with a $\mathrm{BMI}<18.5$ $\mathrm{kg} / \mathrm{m}^{2}$, compared to participants with a BMI of 18.5 to $25 \mathrm{~kg} / \mathrm{m}^{2}$. Among HNC subtypes, this association was strongest for OCC and OHPC. The association between BMI at age 20 and $\mathrm{HNC}$ risk appeared to be positive.

\section{Conclusions}

In this large prospective cohort study, we found an inverse association between $\mathrm{BMI}$ at baseline and $\mathrm{HNC}$ risk. For $\mathrm{BMI}$ at age 20, however, a positive rather than inverse association was found. 


\section{Introduction}

Worldwide and in Europe, head and neck cancer (HNC) is the seventh most common type of cancer, including malignancies in the oral cavity, pharynx, and larynx. ${ }^{1,2}$ Established risk factors for HNC are cigarette smoking, alcohol consumption and human papillomavirus (HPV) infection. ${ }^{3,4}$ A low body mass index (BMI) has also been associated with HNC risk, but this association remains to be clarified. In 2007, the World Cancer Research Fund (WCRF) concluded that data regarding the association between body fatness and HNC risk were insufficient to allow any conclusions to be drawn. ${ }^{3}$ Several case-control studies investigated the association between BMI and HNC and mostly found inverse associations. However, since case-control studies are prone to bias, it remains unclear whether the results represent a true inverse association between BMI and HNC or an association due to reverse causality, confounding or effect modification. ${ }^{3,5-7}$ Recently, three prospective cohort studies investigated the association between BMI and HNC risk. In the Prostate, Lung, Colorectal, and Ovarian (PLCO) cohort $^{8}, \mathrm{BMI}$ at different time points in life was not associated with HNC risk. The Cancer Prevention Study-II (CPS-II) cohort showed no association between BMI and HNC incidence either, although BMI was inversely associated with HNC mortality in smokers. ${ }^{9}$ The National Institutes of Health-AARP (NIH-AARP) Diet and Health Study ${ }^{10}$ found that HNC risk was inversely associated with leanness among current smokers, and concluded that the association between leanness and HNC risk may be due to effect modification by smoking.

Given the current evidence, it remains critical to study the association between $\mathrm{BMI}$ and $\mathrm{HNC}$ risk in prospective cohort studies with comprehensive adjustment for smoking. We therefore examined the association with BMI for HNC and the most frequent HNC subtypes ${ }^{11}-$ i.e., oral cavity cancer (OCC), oro-/hypopharyngeal cancer (OHPC), and laryngeal cancer (LC)-within the large prospective Netherlands Cohort Study (NLCS). In addition to BMI at study baseline, we also studied the effects of BMI at age 20 years and change in BMI during adulthood on HNC risk. Finally, we investigated the association of BMI with HNC risk according to smoking status and alcohol consumption. 


\section{Methods}

\section{Study design and population}

The NLCS was initiated in September 1986 and includes 120,852 participants, aged 5569 years at baseline. ${ }^{12}$ The NLCS has been approved by the institutional review board of the TNO Quality of Life Research Institute (Zeist, The Netherlands) and the Medical Ethics Committee of Maastricht University (Maastricht, The Netherlands). All methods were carried out in accordance with the approved guidelines. All cohort members consented to participate in the study by completing and returning the selfadministered questionnaire.

We used the case-cohort design for efficiency in data processing and follow-up. ${ }^{13}$ Cases were identified from the entire cohort, whereas the number of person-years at risk for the entire cohort was estimated using a subcohort of 5,000 people who were randomly sampled from the total cohort at baseline. Follow-up for cancer incidence was done by record linkage to the Netherlands Cancer Registry (NCR) and the nationwide network and pathology registry (PALGA). ${ }^{14}$ Follow-up for vital status of the subcohort was nearly $100 \%$ complete after 20.3 years and the completeness of cancer follow-up is estimated to be $\geq 96 \%$. ${ }^{15}$

We excluded cohort members with prevalent cancer other than skin cancer at baseline (Figure 6.1). Participants with incomplete/inconsistent dietary data or missing data on confounding variables (see below) were also excluded from analysis. ${ }^{16,17}$ Only microscopically confirmed first occurrences of squamous cell carcinomas were included. ${ }^{1,3}$ These comprise nearly all malignancies of the mouth, pharynx, and larynx.

Data for statistical analysis were available for 3,980 subcohort members and 411 incident cases of the selected HNC subtypes (Figure 6.1). HNC subtypes were classified as proposed by Hashibe et al. ${ }^{18}$, according to the International Classification of Diseases for Oncology (ICD-O-3) ${ }^{19}$ (Table 6.1). Of the 411 HNC cases, 127 were oral cavity cancer (OCC), 84 were oro-/hypopharyngeal cancer (OHPC), three were oral cavity/pharynx unspecified or overlapping (only included in analyses of HNC overall), and 197 were laryngeal cancer (LC) cases.

Table 6.1. Subclassification of subtypes of head-neck cancer (HNC) as proposed by Hashibe et al. ${ }^{18}$, according to the International Classification of Diseases for Oncology, version 3 (ICD-O-3) ${ }^{19}$.

\begin{tabular}{|c|c|}
\hline HNC-subtype & ICD-0-3 \\
\hline \multirow[t]{2}{*}{ Oral cavity cancer (OCC) } & C003-009, C020-C023, C030-C031, C039-C041,C048- \\
\hline & $\mathrm{C} 050, \mathrm{C} 060-\mathrm{C062}, \mathrm{CO68-C069}$ \\
\hline Oro-/hypopharyngeal cancer (OHPC) & $\begin{array}{l}\mathrm{C} 019, \mathrm{C} 024, \mathrm{C} 051-\mathrm{C} 052, \mathrm{C} 090-\mathrm{C} 091, \mathrm{C} 098-\mathrm{C} 104, \mathrm{C} 108- \\
\mathrm{C} 109, \mathrm{C} 129-\mathrm{C} 132, \mathrm{C} 138-\mathrm{C} 139\end{array}$ \\
\hline Oral cavity, pharynx unspecified or overlapping cancer (USC) & C028-C029, C058-C059, C140-C142, C148 \\
\hline Laryngeal cancer (LC) & С $320-C 329$ \\
\hline
\end{tabular}




\begin{tabular}{|c|c|c|c|c|}
\hline \multicolumn{5}{|c|}{ Netherlands Cohort Study on diet and cancer $(120,852)$} \\
\hline$\downarrow$ & & & $\downarrow$ & \\
\hline $\begin{array}{l}\text { Subcohort, randomly } \\
\text { sampled from total cohort }\end{array}$ & \multicolumn{4}{|c|}{ Record linkage with Netherlands Cancer Registry and PALGA } \\
\hline$\underset{5,000}{\downarrow}$ & $\begin{array}{l}\downarrow \\
\text { HNC overall }^{a}\end{array}$ & $\begin{array}{c}\downarrow \\
\text { Oral cavity } \\
\text { cancer (OCC) }\end{array}$ & $\begin{array}{l}\qquad \\
\downarrow \\
\text { Oro-/hypopharyngeal } \\
\text { cancer (OHPC) }\end{array}$ & $\begin{array}{l}\downarrow \\
\downarrow \\
\text { Laryngeal } \\
\text { cancer (LC) }\end{array}$ \\
\hline & 552 & 154 & 124 & 271 \\
\hline \multicolumn{5}{|c|}{ Exclusion of participants with prevalent cancer at baseline } \\
\hline $\begin{array}{c}\downarrow \\
4,774\end{array}$ & $\underset{515}{\downarrow}$ & $\begin{array}{c}\downarrow \\
149\end{array}$ & $\begin{array}{c}\downarrow \\
109\end{array}$ & $\begin{array}{c}\downarrow \\
254\end{array}$ \\
\hline \multicolumn{5}{|c|}{ Exclusion of participants with missing data on BMI } \\
\hline $\begin{array}{c}\downarrow \\
4,558^{b} \\
3,901^{c}\end{array}$ & $\begin{array}{c}\downarrow \\
491 \\
408\end{array}$ & $\begin{array}{c}\downarrow \\
140 \\
119\end{array}$ & $\begin{array}{c}\downarrow \\
103 \\
82\end{array}$ & $\begin{array}{c}\downarrow \\
245 \\
205\end{array}$ \\
\hline \multicolumn{5}{|c|}{ Exclusion of participants with incomplete or inconsistent dietary data } \\
\hline $\begin{array}{c}\downarrow \\
4,293^{b} \\
3,669^{c}\end{array}$ & $\begin{array}{c}\downarrow \\
453 \\
377\end{array}$ & $\begin{array}{c}\downarrow \\
133 \\
113\end{array}$ & $\begin{array}{l}\downarrow \\
96 \\
75\end{array}$ & $\begin{array}{c}\downarrow \\
221 \\
187\end{array}$ \\
\hline \multicolumn{5}{|c|}{ Exclusion of participants with missing data on confounders } \\
\hline $\begin{array}{c}\downarrow \\
3,980^{b} \\
3,417^{c}\end{array}$ & $\begin{array}{c}\downarrow \\
411 \\
341\end{array}$ & $\begin{array}{c}\downarrow \\
127 \\
107\end{array}$ & $\begin{array}{l}\downarrow \\
84 \\
66\end{array}$ & $\begin{array}{c}\downarrow \\
197 \\
166\end{array}$ \\
\hline
\end{tabular}

Figure 6.1. Flow diagram of the number of subcohort members and cases on whom the analyses were based. Abbreviation PALGA: nationwide network and registry of histopathology and cytopathology in the Netherlands. ${ }^{a}$ Oral cavity cancer; oro-/hypopharyngeal cancer; oral cavity, pharynx unspecified or overlapping cancer; laryngeal cancer. ${ }^{\mathrm{b}}$ The number of subcohort members or cases used in analyses of BMI at baseline.

${ }^{\mathrm{C}}$ The numbers of subcohort members or cases used in analyses of BMI at age 20 and change in BMI.

\section{Questionnaire data}

At baseline, all participants completed a self-administered questionnaire about habitual dietary intake, lifestyle habits, and other cancer risk factors, including weight, height, and weight at age 20 years. We asked detailed questions about alcohol consumption and cigarette smoking, as described previously. ${ }^{20}$ Data were key-entered and processed in a standardized manner, blinded with respect to case/subcohort status in order to minimize observer bias in coding and data interpretation.

$\mathrm{BMI}$ at baseline and $\mathrm{BMI}$ at age 20 years were calculated using weight at baseline and weight at 20 years, respectively, divided by height at baseline squared $\left(\mathrm{kg} / \mathrm{m}^{2}\right)$. We classified BMI at baseline according to the World Health Organization (WHO) standard categories: <18.5 (underweight), 18.5 to $<25$ (normal weight), 25 to $<30$ (overweight), and $\geq 30 \mathrm{~kg} / \mathrm{m}^{2}$ (obese). For BMI at age 20 years, categories were $<20.0,20.0$ to $<21.5$, 21.5 to $<23,23$ to $<25$, and $\geq 25 \mathrm{~kg} / \mathrm{m}^{2}$. We did not use WHO categories here because of few obese cases at the age of 20 years; this classification has been used before in other NLCS analyses. ${ }^{21}$ Change in BMI since age 20 years was calculated as BMI at 
baseline minus BMI at age 20 years and was classified as $<0,0$ to $<4,4$ to $<8$, and $\geq 8$ $\mathrm{kg} / \mathrm{m}^{2}{ }^{21}$ Participants with missing values for $\mathrm{BMI}$ at baseline were excluded from all analyses; subjects with missing values for $\mathrm{BMI}$ at age 20 years were excluded from the analyses of BMI at age 20 years and change in BMI (Figure 6.1).

\section{Statistical analysis}

The Cox proportional hazards model was used to estimate age- and sex-adjusted and multivariable-adjusted incidence rate ratios (RR) and corresponding $95 \%$ confidence intervals (CI). Person-years at risk were calculated from baseline until diagnosis of HNC, death, emigration, loss to follow-up, or end of follow-up, whichever occurred first. We analyzed $\mathrm{BMI}$ at baseline, $\mathrm{BMI}$ at age 20 years and change in $\mathrm{BMI}$ since age 20 years as described above. For continuous analyses, we used $1 \mathrm{~kg} / \mathrm{m}^{2}$ as increment in BMI.

To evaluate possible reverse causality, we categorized cases according to the year of follow-up in which they were diagnosed and evaluated BMI of HNC cases during the follow-up period. In addition, we used an independent samples t-test to test for statistical significance of differences between the mean BMI of HNC cases diagnosed during the first two years and cases diagnosed later in follow-up. Based on these results (Table 6.2), we decided to include the total follow-up time in our analyses. We also performed sensitivity analyses regarding the association between BMI at baseline and risk of HNC overall by excluding the first two years of follow-up.

The predefined confounders were age (years); sex; alcohol consumption (grams/day); and cigarette smoking (status (never/former/current), number of cigarettes smoked daily, and number of smoking years). We considered the following potential confounders: level of education; non-occupational physical activity; energyintake; consumption of total vegetables, total fruits, fish, red meat, and meat products (all grams/day); and family history of HNC. ${ }^{3,22}$ None of these variables changed the RR for BMI (continuous) for HNC overall or any of the HNC subtypes by $>10 \%$ when including them in the model. Therefore, the final model included only the predefined confounders. Analyses of change in BMI were also adjusted for BMI at age 20 years. When adjusting for cigarette smoking frequency and duration, we centered these continuous variables as proposed by Leffondré et al. ${ }^{23}$

We assessed tests for linear dose-response trends by fitting ordinal exposure variables as continuous terms. Standard errors were estimated using the robust HuberWhite sandwich estimator to account for additional variance due to sampling from the cohort. The proportional hazards assumption was assessed using the scaled Schoenfeld residuals. ${ }^{24}$ If there was an indication for violation of the assumption for a variable, it was further investigated by adding a time-varying covariate for that variable to the 
Table 6.2. BMI in head-neck cancer (HNC) cases according to sex and time between baseline and HNC diagnosis; Netherlands Cohort Study, 1986-2006

\begin{tabular}{|c|c|c|c|c|}
\hline \multirow[b]{3}{*}{ Cases $^{a}$} & \multicolumn{4}{|c|}{ Head-neck cancer overall } \\
\hline & \multirow[b]{2}{*}{ No. of cases } & \multicolumn{3}{|c|}{ BMI $\left(\mathrm{kg} / \mathrm{m}^{2}\right)$} \\
\hline & & Mean & SD & $P$ value \\
\hline All cases & 411 & 24.8 & 2.7 & \\
\hline Men & 318 & 25.0 & 2.5 & \\
\hline Women & 93 & 24.3 & 3.2 & \\
\hline \multicolumn{5}{|c|}{ Year of follow-up } \\
\hline $0-2$ & 33 & 24.9 & 2.2 & \\
\hline$>2$ & 378 & 24.8 & 2.8 & $0.96^{\mathrm{b}}$ \\
\hline \multicolumn{5}{|c|}{ Categories year of follow-up } \\
\hline $0-2$ & 33 & 24.9 & 2.2 & \\
\hline$>2-4$ & 40 & 24.8 & 3.0 & \\
\hline$>4-6$ & 49 & 24.8 & 3.1 & \\
\hline$>6-8$ & 44 & 24.7 & 2.4 & \\
\hline$>8-10$ & 47 & 24.6 & 2.9 & \\
\hline$>10-12$ & 39 & 24.6 & 3.2 & \\
\hline$>12-14$ & 44 & 24.8 & 2.9 & \\
\hline$>14-16$ & 47 & 25.1 & 2.6 & \\
\hline$>16-18$ & 37 & 25.2 & 2.5 & \\
\hline$>18$ & 31 & 24.9 & 2.3 & \\
\hline
\end{tabular}

model. We performed analyses for HNC and all HNC subtypes using a time-varying covariate for current smoking, as described before. ${ }^{25}$

To determine whether sex, cigarette smoking, or alcohol consumption possibly modify the association of BMI with risk of HNC overall, we estimated RRs in strata of these exposures. Tests for interaction were performed with BMI on a continuous scale and $P$ values for interaction were assessed by including cross-product terms in the models and performing a Wald test. We performed analyses in strata of alcohol consumption (abstainers; consuming $>0$ to 15 grams ethanol/day; consuming $\geq 15$ grams ethanol/day) and cigarette smoking status (never/former/current). Alcohol consumption and cigarette smoking were mutually adjusted in these models.

All reported $P$ values were based on two-sided tests and considered statistically significant if $<0.05$. Analyses were done using the Stata 13.1 statistical software package (StataCorp, College Station, Texas, USA). 


\section{Results}

\section{Baseline characteristics}

The mean BMI at baseline of subcohort members $\left(25.0 \mathrm{~kg} / \mathrm{m}^{2}\right)$ and cases $\left(24.8 \mathrm{~kg} / \mathrm{m}^{2}\right)$ was slightly lower in HNC cases (Table 6.3). There was a minor difference between subcohort members and cases regarding BMI at age 20 years $\left(21.5\right.$ and $21.9 \mathrm{~kg} / \mathrm{m}^{2}$, respectively), as well as with respect to change in BMI since age 20 years (plus 3.5 and $3.0 \mathrm{~kg} / \mathrm{m}^{2}$, respectively). Among subcohort members and cases, men generally had a similar mean BMI, whereas female cases had a considerably lower BMI at baseline than both male cases and female subcohort members. Notable characteristics with regard to cigarette smoking and alcohol consumption have been described previously ${ }^{20}$.

To examine possible reverse causation, we evaluated BMI at baseline of HNC cases during the follow-up period. As we expected, there was no clear pattern in BMI at baseline among HNC cases diagnosed in the course of 20.3 years of follow-up (Table 6.2). HNC overall cases diagnosed after the second year of follow-up ( $N=378$ ) had a mean $\mathrm{BMI}$ at baseline of $24.8 \mathrm{~kg} / \mathrm{m}^{2}$, whereas cases diagnosed during the first two years of follow-up $(N=33)$ had a mean $B M I$ of $24.9 \mathrm{~kg} / \mathrm{m}^{2}$, a non-statistically significant difference.

\section{Main analyses}

Results from age- and sex-adjusted and multivariable-adjusted analyses showed mostly inverse associations between BMI at baseline and risk of HNC overall and HNC subtypes, although these associations were generally somewhat weaker in multivariable-adjusted analyses (Table 6.4).

$\mathrm{BMI}$ at baseline was inversely associated with risk of HNC overall, with a multivariable-adjusted RR of 3.31 (95\% confidence interval $(\mathrm{Cl})$ 1.40-7.82) for subjects with a $\mathrm{BMI}<18.5 \mathrm{~kg} / \mathrm{m}^{2}$, whereas participants with a $\mathrm{BMI} \geq 30 \mathrm{~kg} / \mathrm{m}^{2}$ had a RR of 0.48 (95\% Cl 0.22-1.03), both compared to participants with a BMI of 18.5 to $25 \mathrm{~kg} / \mathrm{m}^{2}$ (Table 6.4). The association between BMI at baseline and risk of HNC overall was comparable for men and women and no statistically significant interaction with sex was found $(P=0.29)$ for $\mathrm{BMI}$ on a continuous scale. Sensitivity analyses showed essentially similar results after exclusion of the first two years of follow-up. We investigated whether the subgroup with underweight at baseline had specific characteristics with regard to smoking and alcohol consumption, since this might have biased the results, but this group was very heterogeneous with regard to these lifestyle aspects. 
Among HNC subtypes, BMI at baseline was in general inversely associated with HNC risk as well, with statistically significant associations in OCC (multivariableadjusted RR comparing participants with a BMI $<18.5 \mathrm{~kg} / \mathrm{m}^{2}$ to those with a $\mathrm{BMI}$ of 18.5 to $25: 4.49,95 \% \mathrm{Cl} 1.45-13.93$ ) and OHPC (RR: $4.96,95 \% \mathrm{Cl} 1.34-18.33$ ) but not LC (RR: $1.25,95 \% \mathrm{Cl} 0.15-10.31$ ) (Table 6.4). For LC, however, a statistically significant interaction with sex $(P=0.01)$ was found, with a decreased risk of LC per $\mathrm{kg} / \mathrm{m}^{2}$ increase in $\mathrm{BMI}$ in women (RR: $0.83,95 \% \mathrm{Cl}$ 0.71-0.97), but the number of female cases was small $(N=14)$. We performed sensitivity analyses with only men $(N=183)$ in categories of BMI at baseline because of this interaction, but these results showed the same pattern as the results for men and women combined (data not shown).

In contrast to the association between BMI at baseline and HNC risk, the association between $\mathrm{BMI}$ at age 20 and $\mathrm{HNC}$ risk appeared to be positive rather than inverse, with statistically significant associations on the continuous scale (Table 6.5). Furthermore, point estimates regarding the association between change in BMI since the age of 20 years and HNC risk mostly indicated an inverse association. In addition, we found an interaction between sex and BMI at age 20 years for HNC overall. RRs regarding $\mathrm{BMI}$ at age 20 years appeared slightly stronger in multivariable-adjusted analyses compared with age- and sex-adjusted analyses, whereas associations between change in BMI and HNC risk showed both stronger and weaker RRs in multivariable-adjusted analyses (Table 6.5).

\section{Stratified analyses}

No statistically significant interaction was found between $\mathrm{BMI}$ at baseline and cigarette smoking ( $P$ interaction=0.86) for $\mathrm{HNC}$ overall, nor for $\mathrm{BMI}$ at age 20 or change in $\mathrm{BMI}$ and cigarette smoking (Table 6.6). A statistically significant interaction was found for both $\mathrm{BMI}$ at baseline and at age 20 and alcohol consumption; stratified analyses showed a consistent pattern of the lowest relative risks of HNC overall for BMI at baseline, $\mathrm{BMI}$ at age 20 , and change in $\mathrm{BMI}$ in non-drinkers. 


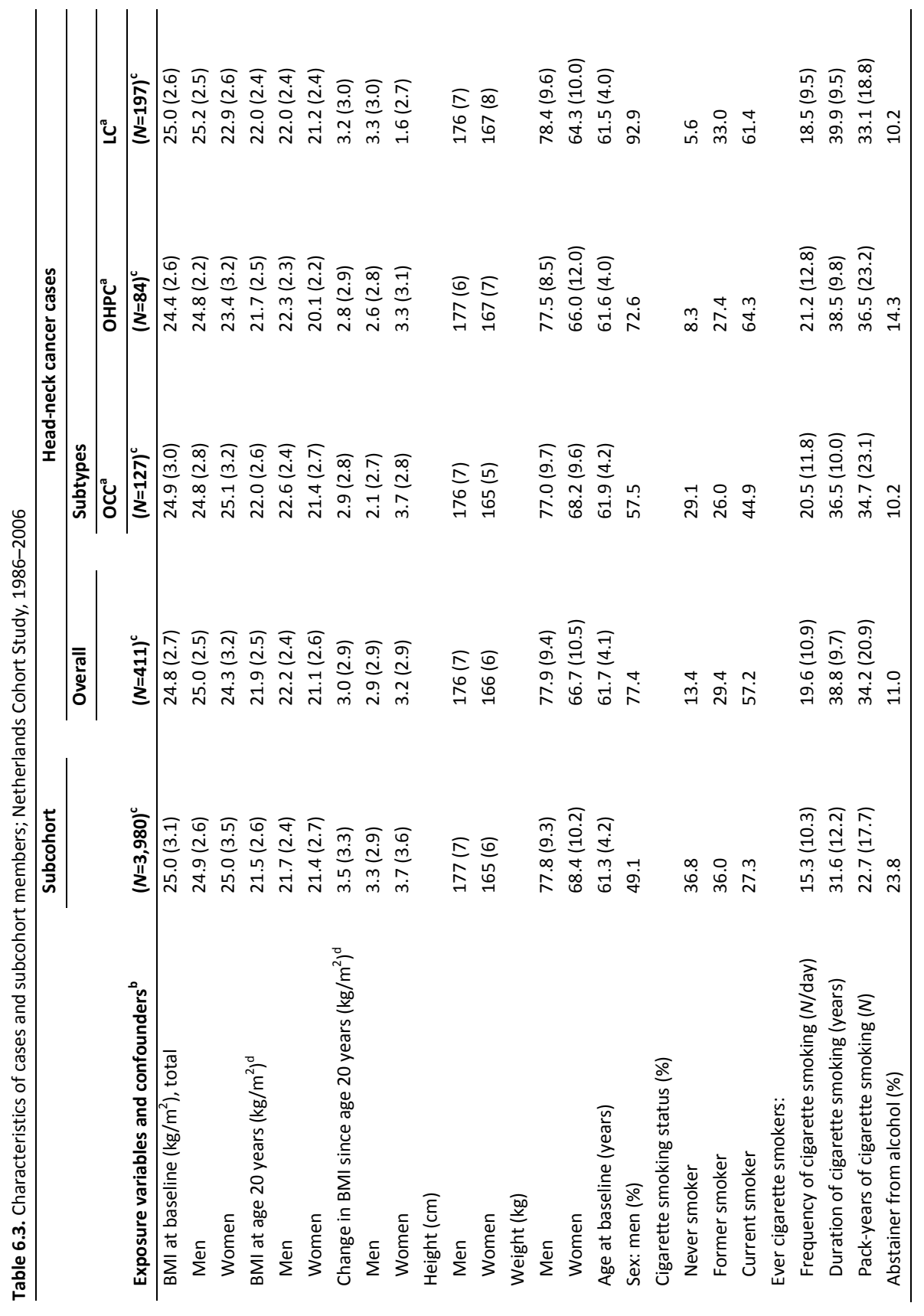




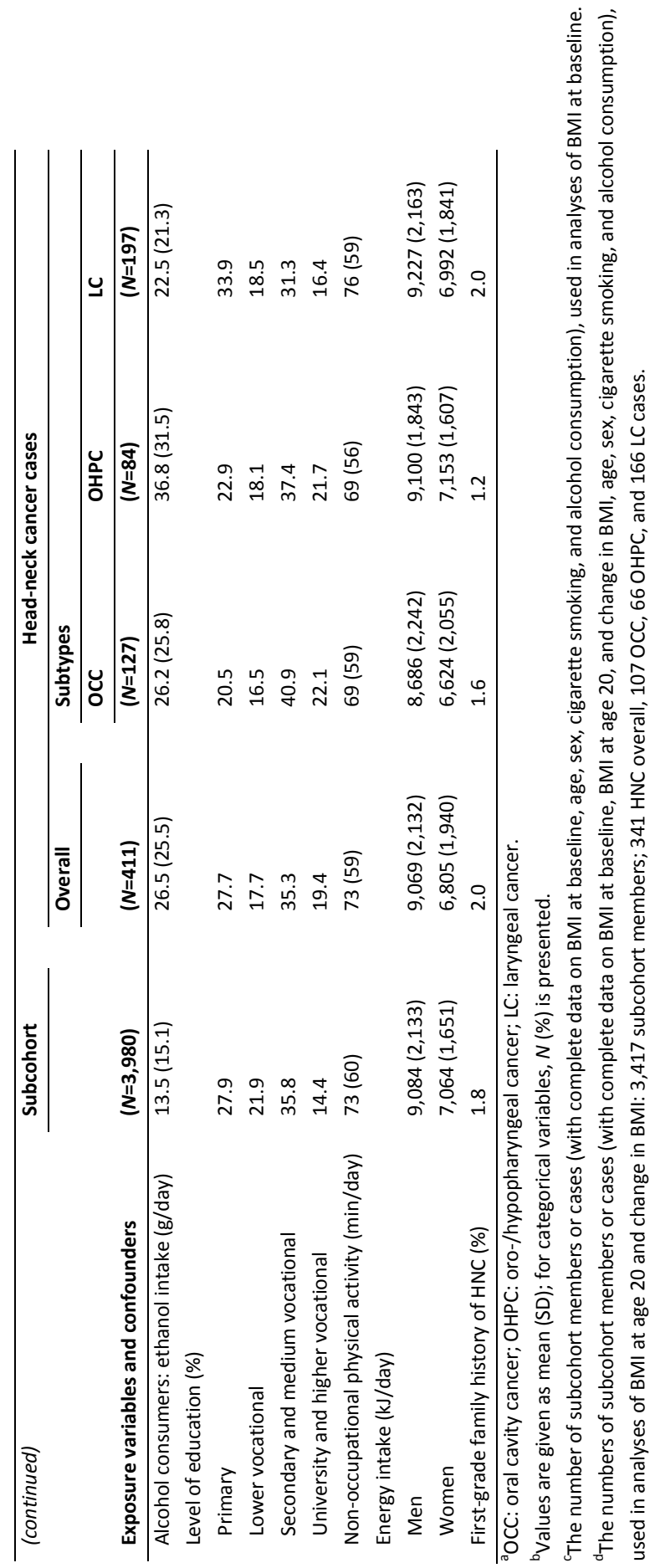




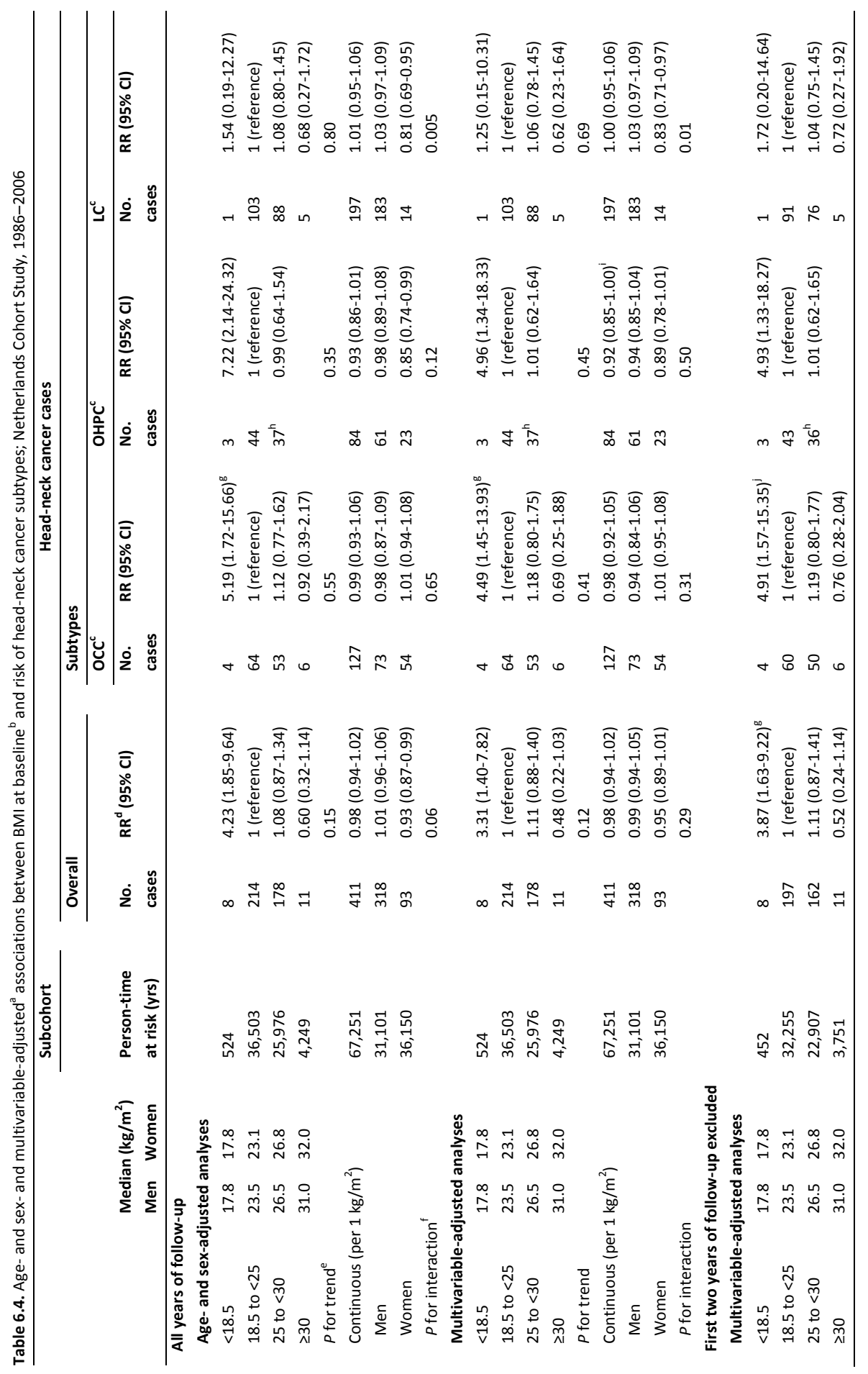




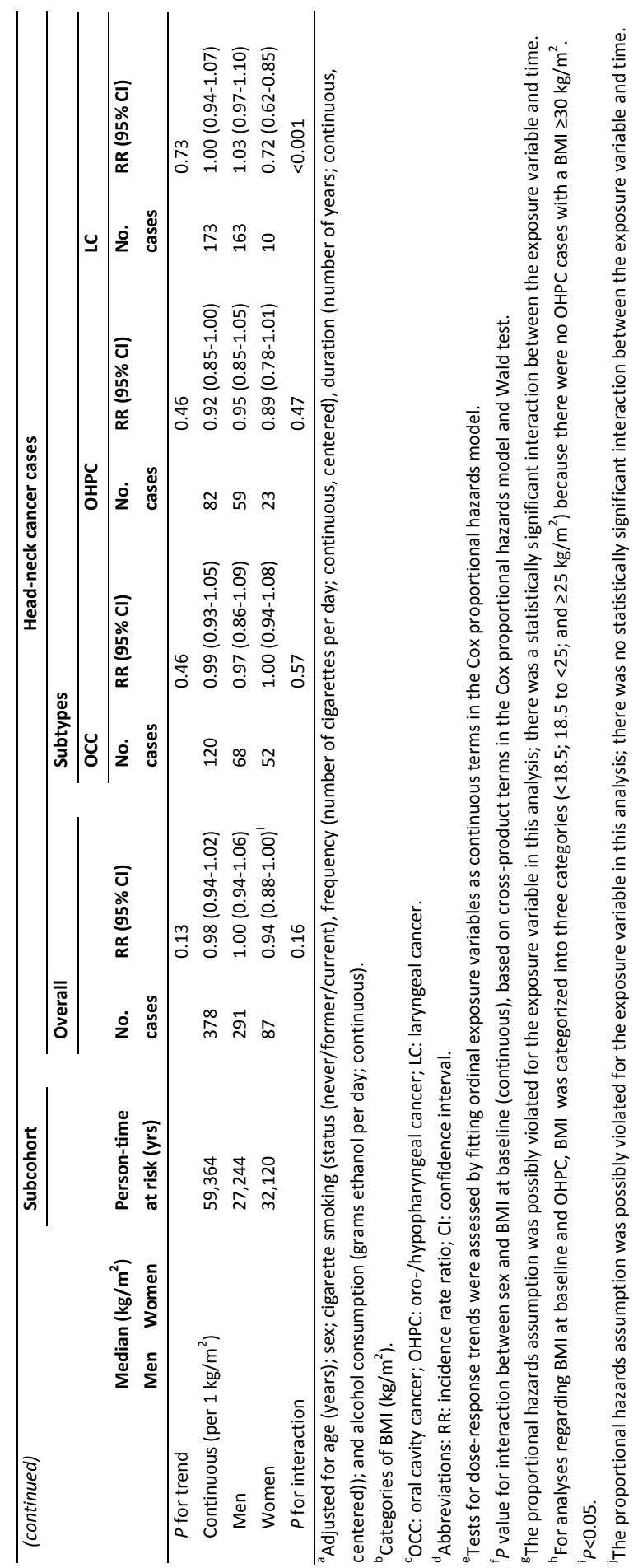




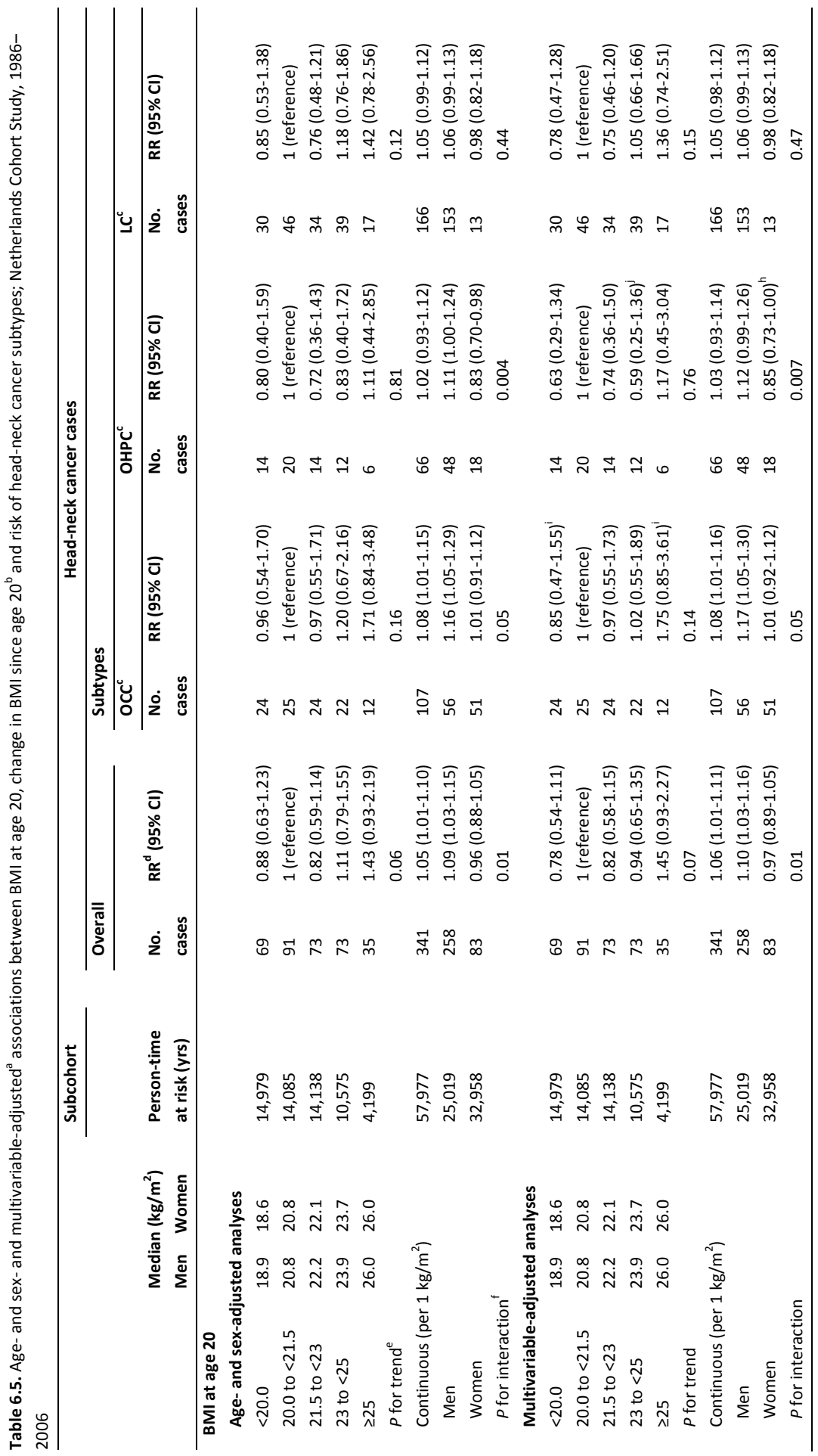




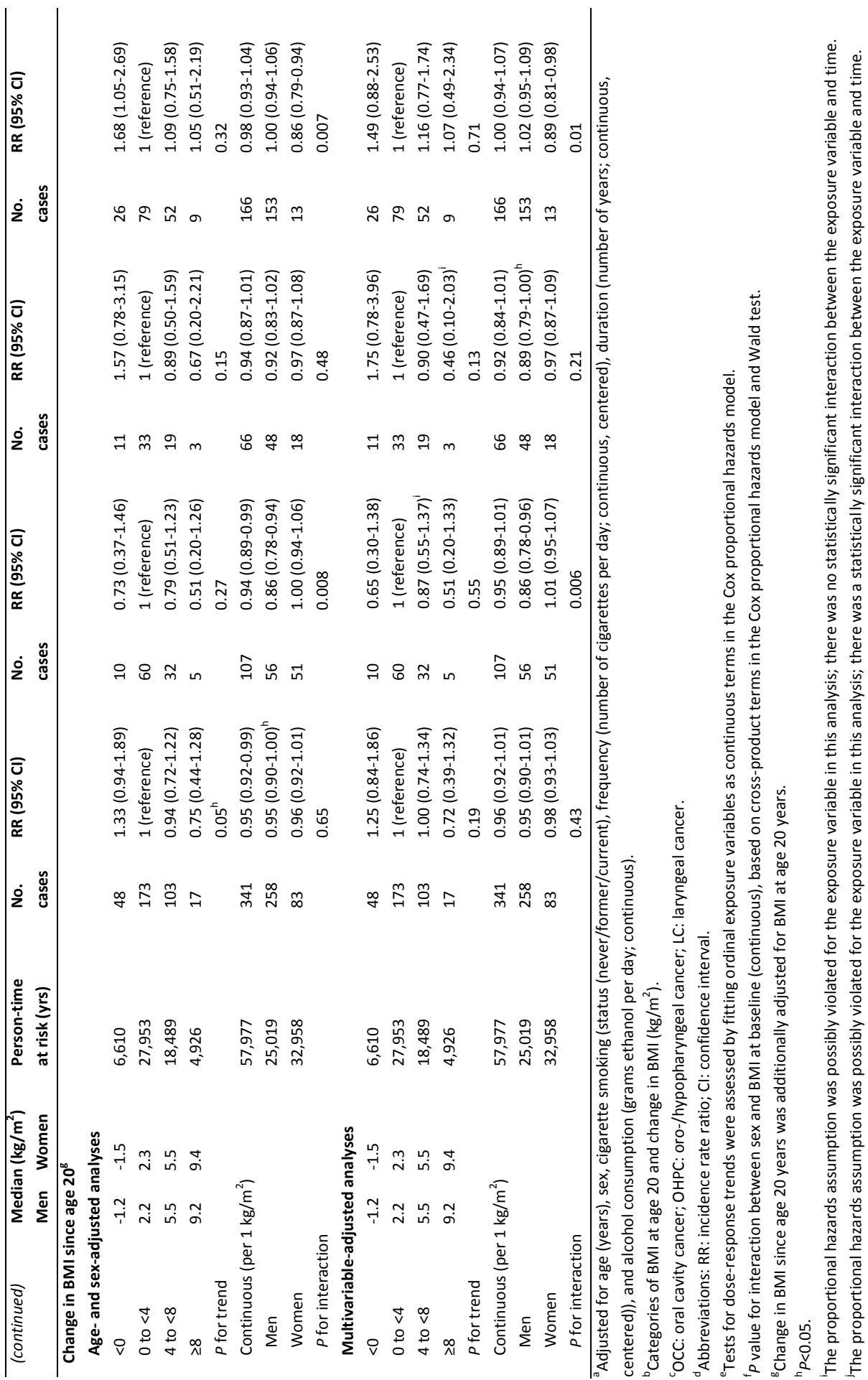


Table 6.6. Multivariable-adjusted ${ }^{a}$ associations between $\mathrm{BMI}^{\mathrm{b}}$ and risk of head-neck cancer overall, stratified by cigarette smoking status and alcohol consumption; Netherlands Cohort Study, 1986-2006

\begin{tabular}{|c|c|c|c|c|}
\hline \multicolumn{5}{|c|}{ Head-neck cancer overall } \\
\hline Cigarette smoking & Never & Former & Current & $\begin{array}{l}P \text { inter- } \\
\text { action }^{c}\end{array}$ \\
\hline \multicolumn{5}{|l|}{$\mathrm{BMI}$ at baseline } \\
\hline Cases/ person-time at risk (years) & $55 / 26,337$ & $121 / 23,911$ & $235 / 17,003$ & 0.86 \\
\hline $\operatorname{RR}^{d}(95 \% \mathrm{Cl})$ & $1.00(0.91-1.09)$ & $0.98(0.90-1.06)$ & $0.97(0.92-1.02)$ & \\
\hline \multicolumn{5}{|l|}{ BMI at age 20} \\
\hline Cases/ person-time at risk (years) & $46 / 23,259$ & $104 / 20,440$ & $191 / 14,278$ & 0.85 \\
\hline $\mathrm{RR}(95 \% \mathrm{Cl})$ & $1.10(1.00-1.21)^{f}$ & $1.05(0.96-1.14)$ & $1.06(0.99-1.13)$ & \\
\hline \multicolumn{5}{|l|}{ Change in BMI since age $20^{\mathrm{e}}$} \\
\hline Cases/ person-time at risk (years) & $46 / 23,259$ & $104 / 20,440$ & $191 / 14,278$ & 0.94 \\
\hline RR $(95 \% \mathrm{Cl})$ & $0.96(0.87-1.05)$ & $0.97(0.88-1.07)$ & $0.96(0.90-1.02)$ & \\
\hline Alcohol consumption & Non-drinkers & $>0-15$ (g/day) & $\geq 15$ (g/day) & \\
\hline \multicolumn{5}{|l|}{ BMI at baseline } \\
\hline Cases/ person-time at risk (years) & $45 / 15,880$ & $156 / 34,959$ & $210 / 16,413$ & $0.05^{f}$ \\
\hline $\mathrm{RR}(95 \% \mathrm{Cl})$ & $0.86(0.78-0.95)$ & $1.01(0.96-1.07)$ & $0.99(0.93-1.05)$ & \\
\hline \multicolumn{5}{|l|}{ BMI at age 20} \\
\hline Cases/ person-time at risk (years) & $41 / 13,675$ & $136 / 30,240$ & $164 / 14,062$ & 0.01 \\
\hline $\mathrm{RR}(95 \% \mathrm{Cl})$ & $0.90(0.81-1.00)^{\mathrm{g}}$ & $1.08(1.00-1.16)^{f}$ & $1.08(1.01-1.16)$ & \\
\hline \multicolumn{5}{|l|}{ Change in $\mathrm{BMI}$ since age $20^{\mathrm{e}}$} \\
\hline Cases/ person-time at risk (years) & $41 / 13,675$ & $136 / 30,240$ & $164 / 14,062$ & 0.77 \\
\hline $\mathrm{RR}(95 \% \mathrm{Cl})$ & $0.88(0.79-0.99)$ & $0.99(0.93-1.06)$ & $0.96(0.90-1.03)$ & \\
\hline
\end{tabular}

${ }^{a}$ Mutually adjusted for age (years); sex; cigarette smoking (status (never/former/current), frequency (number of cigarettes per day; continuous, centered), duration (number of years; continuous, centered)); and alcohol consumption (grams ethanol per day; continuous).

${ }^{\mathrm{b}}$ Continuous (per $1 \mathrm{~kg} / \mathrm{m}^{2}$ increment).

${ }^{\mathrm{c}} P$ value for interaction based on cross-product terms in the Cox proportional hazards model and Wald test.

${ }^{\mathrm{d}}$ Abbreviations: RR: incidence rate ratio; $\mathrm{Cl}$ : confidence interval.

${ }^{\mathrm{e}}$ Change in BMI since age 20 years was additionally adjusted for BMI at age 20 years.

${ }^{f} P<0.05$.

${ }^{\mathrm{g}} P>0.05$. 


\section{Discussion}

In this large prospective cohort study, we found an inverse association between BMI at baseline and risk of HNC overall. Among HNC subtypes, BMI at baseline showed the strongest inverse association with OCC and OHPC. For BMI at age 20, on the other hand, we found a positive rather than inverse association, whereas the association between change in BMI since the age of 20 years and HNC risk appeared to be inverse again. Finally, there was effect modification by alcohol consumption in our study, with the lowest risks of $\mathrm{HNC}$ overall for $\mathrm{BMI}$ at baseline, $\mathrm{BMI}$ at age 20 , and change in $\mathrm{BMI}$ in non-drinkers.

\section{$B M I$ and risk of HNC and HNC subtypes}

Previous studies showed mixed results regarding BMI and HNC risk. Case-control studies largely indicated an inverse association between BMI and HNC risk ${ }^{3}$, but a systematic literature review by the $\mathrm{WCRF}^{3}$ concluded that data regarding the association between body fatness and HNC risk-based on case-control studies-were insufficient to allow conclusions to be drawn. Since then, a large pooled analysis ${ }^{5}$ of 17 case-control studies with 12,716 cases and 17,438 controls showed that leanness (BMI $<18.5 \mathrm{~kg} / \mathrm{m}^{2}$ ) was associated with increased HNC risk, regardless of smoking and drinking status. Furthermore, three prospective cohort studies examined the association between BMI and HNC risk. The CPS-II cohort ${ }^{9}$ included $340 \mathrm{HNC}$ cases and showed no association between BMI and HNC incidence. There was no effect modification by smoking status. In the PLCO cohort ${ }^{8}$, with 177 cases, neither BMI at different time points in life nor changes in BMI were associated with HNC risk. Recently, the NIH-AARP Diet and Health Study ${ }^{10}$, which comprised 779 cases, showed evidence for an inverse relationship between BMI at baseline and HNC risk, in particular OCC and OHPC, but none of the associations were statistically significant. In addition, BMI at earlier ages showed no association with HNC risk. When stratified by smoking, the inverse association was only observed among current (and not former) smokers (hazard ratio 0.76 per $5 \mathrm{~kg} / \mathrm{m}^{2}$ increase, $95 \% \mathrm{Cl} 0.63-0.93$ ); also, the association diminished as initial years of follow-up were excluded. None of the three cohort studies investigated effect modification by alcohol intake.

The results from our prospective cohort study partly confirm findings from previous-both case-control and prospective-studies. As most case-control studies and the NIH-AARP cohort study ${ }^{10}$, we also found an inverse association between BMI and HNC risk, and-like NIH-AARP-with strongest associations for OCC and OHPC. The CPS- ${ }^{9}$ and the $\mathrm{PLCO}^{8}$ cohort, on the other hand, did not find an inverse association 
between $\mathrm{BMI}$ and HNC risk. Unlike previous cohort studies ${ }^{8,10}$, we also found a positive association with regard to $\mathrm{BMI}$ at age 20 and $\mathrm{HNC}$ risk, and an interaction with alcohol consumption. Finally, we did not find an interaction with smoking status, although this might have to do with a lack of power (see below).

\section{Possible mechanisms regarding BMI and HNC risk}

The question remains whether the inverse association we found between BMI at baseline and $\mathrm{HNC}$ risk is a true effect by $\mathrm{BMI}$, or an effect based on reverse causality or confounding by smoking, alcohol consumption, or other factors. We cannot clearly explain why we found a positive rather than inverse association between BMI at age 20 and $\mathrm{HNC}$ risk, whilst BMI at baseline was in general inversely associated with HNC risk. Given the contrast in our results regarding the associations between BMI at baseline, BMI at age 20, and HNC risk, it appears that leanness itself is probably not a causal factor in this association. The fact that some associations were weaker-but others stronger-in multivariable-adjusted analyses than in age- and sex-adjusted analyses implies the possibility of residual confounding. Reverse causality might play a role in the association between BMI at baseline and HNC risk. However, sensitivity analyses showed similar results for different periods of follow-up, which makes reverse causality unlikely.

\section{Strengths and limitations}

Strengths of our study are the prospective nature, our large case-number, and the completeness and duration of follow-up. In addition, we had the ability to study HNC subtypes and to adjust for confounders thoroughly. A possible limitation of our study is that the data on BMI in our study are self-reported, which may have led to bias due to misclassification of exposure. BMI at age 20 years was calculated using self-reported weight at age 20 years and this might have introduced recall bias; however, we expect this to be non-differential. Despite thorough adjustment for confounding by smoking and alcohol consumption, we cannot rule out residual confounding, as described above. Furthermore, in stratified analyses, we did not find a statistically significant interaction with regard to cigarette smoking. However, the analysis included only 55 cases among never smokers, mainly females, and there may have been a lack of power to detect a significant interaction. Finally, we lack data on HPV infection. ${ }^{20}$

\section{Conclusions}

In conclusion, we found an inverse association between BMI at baseline and HNC risk in this large cohort study. Among HNC subtypes, this association was strongest for OCC 
and OHPC. For BMI at age 20, however, a positive rather than inverse association was found. Furthermore, associations of BMI with HNC risk may be modified by alcohol consumption. We conclude that leanness itself is probably not a causal factor in the association with HNC. Future studies are warranted for further clarifications of the possible mechanisms involved regarding BMI and HNC risk.

\section{References}

1 Argiris, A., Karamouzis, M. V., Raben, D. \& Ferris, R. L. Head and neck cancer. Lancet 371, 1695-1709 (2008).

2 Ferlay J. et al. GLOBOCAN 2008 v1.2, Cancer Incidence and Mortality Worldwide: IARC CancerBase No. 10 [Internet]. Lyon, France: International Agency for Research on Cancer; 2010. Available from: http://globocan.iarc.fr, accessed on 30/05/2013.

3 World Cancer Research Fund \& American Institute for Cancer Research. Food, Nutrition, Physical Activity and the Prevention of Cancer: a Global Perspective. American Institute for Cancer Research, 2007.

4 IARC. A Review of Human Carcinogens: Biological Agents. Vol. 100B International Agency for Research on Cancer, 2012.

5 Gaudet, M. M. et al. Body mass index and risk of head and neck cancer in a pooled analysis of case-control studies in the International Head and Neck Cancer Epidemiology (INHANCE) Consortium. Int J Epidemiol 39, 1091-1102 (2010).

6 Lubin, J. H. et al. Body mass index, cigarette smoking, and alcohol consumption and cancers of the oral cavity, pharynx, and larynx: modeling odds ratios in pooled case-control data. Am J Epidemiol 171, 1250-1261 (2010).

7 Lubin, J. H. et al. An examination of male and female odds ratios by BMI, cigarette smoking, and alcohol consumption for cancers of the oral cavity, pharynx, and larynx in pooled data from 15 case-control studies. Cancer Causes Control 22, 1217-1231 (2011).

8 Hashibe, M. et al. Tobacco, alcohol, body mass index, physical activity, and the risk of head and neck cancer in the prostate, lung, colorectal, and ovarian (PLCO) cohort. Head Neck 35, 914-922 (2013).

9 Gaudet, M. M. et al. Prospective studies of body mass index with head and neck cancer incidence and mortality. Cancer Epidemiol Biomarkers Prev 21, 497-503 (2012).

10 Etemadi, A. et al. A prospective cohort study of body size and risk of head and neck cancers in the NIH-AARP diet and health study. Cancer Epidemiol Biomarkers Prev 23, 2422-2429 (2014).

11 Dobrossy, L. Epidemiology of head and neck cancer: magnitude of the problem. Cancer Metastasis Rev 24, 9-17 (2005).

12 van den Brandt, P. A. et al. A large-scale prospective cohort study on diet and cancer in The Netherlands. J Clin Epidemiol 43, 285-295 (1990).

13 Barlow, W. E., Ichikawa, L., Rosner, D. \& Izumi, S. Analysis of case-cohort designs. J Clin Epidemiol 52, 1165-1172 (1999).

14 van den Brandt, P. A., Schouten, L. J., Goldbohm, R. A., Dorant, E. \& Hunen, P. M. Development of a record linkage protocol for use in the Dutch Cancer Registry for Epidemiological Research. Int J Epidemiol 19, 553-558 (1990).

15 Goldbohm RA, Van den Brandt PA \& Dorant E. Estimation of the coverage of Dutch municipalities by cancer registries and PALGA based on hospital discharge data. Tijdschr Soc Gezondheidsz, 80-84 (1994).

16 Goldbohm, R. A. et al. Validation of a dietary questionnaire used in a large-scale prospective cohort study on diet and cancer. Eur J Clin Nutr 48, 253-265 (1994).

17 Heinen, M. M., Verhage, B. A., Goldbohm, R. A. \& van den Brandt, P. A. Intake of vegetables, fruits, carotenoids and vitamins $C$ and $E$ and pancreatic cancer risk in The Netherlands Cohort Study. Int J Cancer 130, 147-158 (2012).

18 Hashibe, M. et al. Alcohol drinking in never users of tobacco, cigarette smoking in never drinkers, and the risk of head and neck cancer: pooled analysis in the International Head and Neck Cancer Epidemiology Consortium. J Natl Cancer Inst 99, 777-789 (2007).

19 Fritz, A. G. International Classification of Diseases for Oncology: ICD-O. 3rd edn, World Health Organization, 2000. 
20 Maasland, D. H., van den Brandt, P. A., Kremer, B., Goldbohm, R. A. \& Schouten, L. J. Alcohol consumption, cigarette smoking and the risk of subtypes of head-neck cancer: results from the Netherlands Cohort Study. BMC Cancer 14, 187 (2014).

21 Merry, A. H., Schouten, L. J., Goldbohm, R. A. \& van den Brandt, P. A. Body mass index, height and risk of adenocarcinoma of the oesophagus and gastric cardia: a prospective cohort study. Gut 56, 1503-1511 (2007).

22 Negri, E. et al. Family history of cancer: pooled analysis in the International Head and Neck Cancer Epidemiology Consortium. Int J Cancer 124, 394-401 (2009).

23 Leffondre, K., Abrahamowicz, M., Siemiatycki, J. \& Rachet, B. Modeling smoking history: a comparison of different approaches. Am J Epidemiol 156, 813-823 (2002).

24 Schoenfeld, D. Partial residuals for the proportional hazards regression model. Biometrika 69, 239-241 (1982).

25 Maasland, D. H., van den Brandt, P. A., Kremer, B., Goldbohm, R. A. \& Schouten, L. J. Consumption of vegetables and fruits and risk of subtypes of head-neck cancer in the Netherlands Cohort Study. Int J Cancer 136, E396-409 (2015). 
Chapter 7

Discussion 
In this chapter, we will discuss the main findings of our study regarding the associations between lifestyle factors and the risk of head-neck cancer (HNC) and HNC subtypes. We will put our findings into perspective with the existing evidence and attempt to create an integrated view regarding the etiology of HNC. Furthermore, we will discuss the strengths and limitations of our study. Finally, recommendations for future research will be given.

\section{Main findings}

We studied possible lifestyle factors regarding risk of HNC and HNC subtypes, i.e., oral cavity cancer (OCC), oro-/hypopharyngeal cancer (OHPC), and laryngeal cancer (LC), within the large prospective Netherlands Cohort Study (NLCS). Our study is one of the largest prospective cohort studies on lifestyle factors and HNC risk, in particular HNC subtypes, and our findings-summarized in Table 7.1-therefore add significantly to the existing literature. We will further elaborate on this in the next paragraph.

First, we investigated the association between alcohol consumption, cigarette smoking and risk of HNC (subtypes). We found that both alcohol consumption and cigarette smoking were strongly, independently associated with an increased risk of HNC (Chapter 2). In addition, we found a positive, multiplicative interaction between both factors with regard to HNC risk. The strength of these associations, however, differed among HNC subtypes; OCC was most strongly associated with alcohol consumption but most weakly with cigarette smoking, whereas LC was not statistically significantly associated with alcohol consumption.

Next, we found that total vegetable and fruit consumption was inversely associated with risk of HNC overall and all HNC subtypes (Chapter 3), with the strongest inverse associations for OCC. Total vegetable consumption was inversely associated with risk of HNC overall as well; associations with HNC subtypes were also inverse and were largely similar for OCC, OHPC, and LC. Total fruit consumption was associated with reduced risks of HNC overall and all subtypes; the strongest associations were with OCC. Associations between vegetable and fruit consumption and HNC risk were not clearly modified by cigarette smoking status or alcohol consumption.

In Chapter 4, we examined associations of vitamin and carotenoid intake with HNC risk. Strong inverse associations were found between vitamin C and HNC overall, OCC and OHPC. No clear associations were found for other nutrients and vitamin supplementation; however, most point estimates showed possible protective effects. 


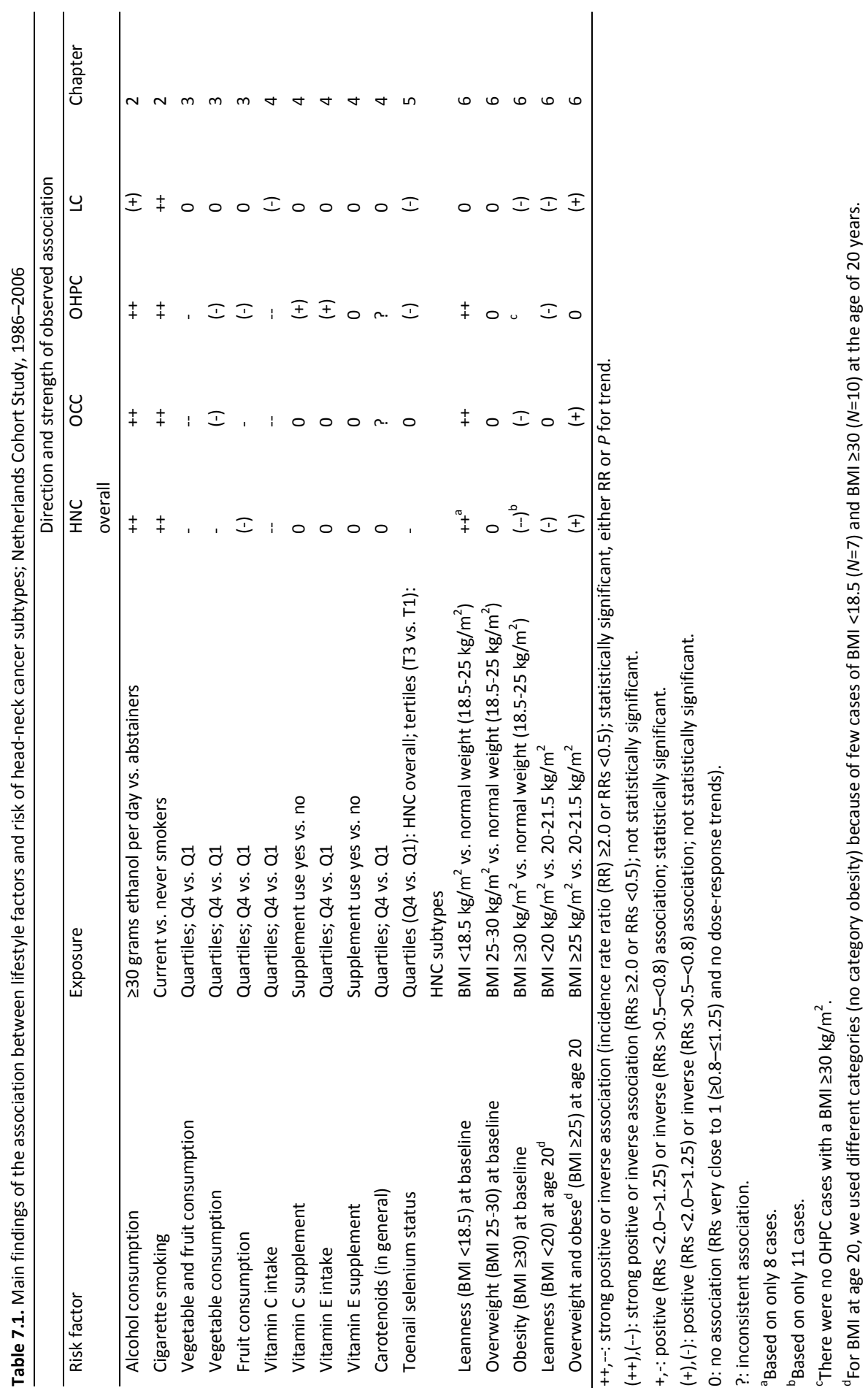


Furthermore, the association between vitamin E intake and the risk on HNC overall was modified by alcohol consumption.

We found an inverse association between toenail selenium level and HNC risk (Chapter 5). Among HNC subtypes, this association was strongest for OHPC and LC. The association between toenail selenium and risk of HNC overall was considerably stronger among men than women. Although there was no clear effect modification by cigarette smoking or alcohol consumption in our study, the association we found was most explicit among current smokers. Associations of toenail selenium status with HNC risk may be modified by gender, cigarette smoking, and alcohol consumption.

Finally, we found an inverse association between BMI at baseline and HNC risk (Chapter 6). Among HNC subtypes, this association was strongest for OCC and OHPC. For $\mathrm{BMI}$ at age 20 , on the other hand, we found a positive rather than inverse association, whereas the association between change in BMI since the age of 20 years and HNC risk appeared to be inverse again. There was effect modification by alcohol consumption in our study, with the lowest risks of HNC overall for BMI at baseline, BMI at age 20 , and change in BMI in non-drinkers.

\section{Interpretation of study findings}

When putting the lifestyle factors we investigated in perspective with regard to HNC risk, what can we say? First, our study confirms that alcohol consumption and cigarette smoking are main risk factors for HNC. ${ }^{1}$ They strongly increase HNC risk, and when used both, the risk is even higher. Alcoholic beverages and acetaldehyde, the main metabolite of ethanol, are classified as a class I carcinogen ${ }^{2}$, and it is plausible that alcohol acts both directly and indirectly-after being metabolized-in HNC carcinogenesis. ${ }^{3,4}$ Cigarette smoking contains numerous carcinogenic substances, e.g., nicotine and benzene, which largely explain the increased HNC risk. ${ }^{5}$ In addition, tobacco carcinogens can solve in alcohol, which may explain the interaction between smoking and alcohol consumption. ${ }^{3,4}$ The differential risk among HNC subtypes may be explained by the larynx having the least direct exposure to alcohol-yet the most to cigarette smoke-compared with the oral cavity and pharynx (next paragraph). ${ }^{4,6}$

Furthermore, we clearly showed that other lifestyle factors significantly influence HNC risk as well. Vegetable and fruit consumption, as well as vitamin C intake, was associated with a significantly decreased HNC risk. The intake of a vitamin C supplement, vitamin $\mathrm{E}$, and carotenoids, however, did not clearly show an association with the risk of developing HNC in our study. Finally, a low toenail selenium status was 
related to an increased $\mathrm{HNC}$ risk, as well as being underweight $\left(\mathrm{BMI}<18.5 \mathrm{~kg} / \mathrm{m}^{2}\right)$ at baseline.

We can thus conclude that nutrition and lifestyle factors other than smoking and alcohol consumption can clearly influence HNC risk. But in which ways does this effect occur? When we look at the big picture of our results, the anticarcinogenic and antioxidant properties of vegetables, fruits, vitamin $C$ and selenium probably play an important role. ${ }^{3,7,8}$ The potential anticarcinogenic properties of numerous components present in vegetables and fruits include vitamins, fiber, folate, flavonoids and carotenoids, some of which are potential antioxidants and might play a role in several processes, such as protection against oxidative stress, normal cell proliferation and differentiation, and DNA repair. ${ }^{3,7}$ Selenium, an essential trace element present in food, probably influences carcinogenesis by its antioxidant properties. ${ }^{8}$

Antioxidants likely counteract oxidative stress, which is amongst others caused by smoking. ${ }^{3}$ However, we showed no clear interaction of the lifestyle factors we studied with smoking (or alcohol); had there been an interaction, we would have expected to see a protective effect of, e.g., vegetables and fruits especially among smokers. This might suggest that nutrition is an important part of a healthy lifestyle and diminishes HNC risk, whether someone is a cigarette smoker and/or alcohol consumer or not. Still, our results might be influenced by power issues, mainly because of the low number of (male) never smokers in our cohort, and epidemiological evidence about potential interaction effects on HNC risk remains therefore inconclusive.

Few cohort studies have investigated lifestyle factors and HNC risk. ${ }^{9-13}$ The National Institutes of Health-AARP (NIH-AARP) Diet and Health cohort $^{13-15}$, with 490,802 United States participants and 787 cases, supports the hypotheses that alcohol consumption and cigarette smoking increase HNC risk, and that total vegetable and fruit intake is associated with reduced HNC risk. However, this large study lacks information on smoking duration, a relevant aspect of smoking behavior. In the prospective European Investigation Into Cancer and Nutrition (EPIC) study ${ }^{9,16}$ with 345,904 subjects and 352 cases (164 oral cavity and pharynx, 84 esophagus, and 104 larynx), intake of fruits and vegetables was also linked with reduced risk of upper aerodigestive tract cancer (UADTC), whereas alcohol increased UADTC risk. Yet, HNC was combined with esophageal cancer in EPIC, which may lead to other overall associations. Chyou et al. ${ }^{10}$ examined diet, alcohol, smoking and UADTC risk in a prospective cohort study among 7,995 Japanese-American men, but with only 92 cases. Again, cigarette smoking and alcohol consumption increased UADTC risk, whereas consumption of fruit was also inversely associated with risk of UADTC. A prospective study of Norwegian men by Kjaerheim et al. ${ }^{12}$ investigated the role of alcohol, tobacco, and dietary factors 
in upper aerogastric tract cancers (UAGT) and had similar findings regarding alcohol, smoking, fruit intake and risk of UAGT. However, the study only included 71 cases (28 oral and pharyngeal, 21 laryngeal, and 22 esophageal cancer cases), analyzed altogether. Lastly, the prospective lowa Women's Health Study ${ }^{11,17}$ investigated dietary factors and UADTC risk in a cohort of 34,691 postmenopausal women, with eventually 169 cases (53 oropharyngeal, 21 laryngeal, 18 nasopharyngeal/salivary, 21 esophageal, and 56 gastric cancer cases), and found that alcohol consumption and smoking increased UADTC risk, whereas intake of fruits and vegetables may reduce risk of UADTC. UADTC subtypes were also investigated, but numbers were too small for statistical testing within individual cancers. Observational studies regarding selenium and HNC risk showed mixed results and mostly had small numbers as well. ${ }^{18-20}$ Finally, three prospective cohort studies - with reasonably large case numbers - investigated the association between BMI and HNC risk, but also showed mixed results. ${ }^{21-23}$

In summary, prospective studies regarding alcohol, smoking, dietary factors and HNC often had few HNC cases ${ }^{10-12}$, did not inform of specific HNC subtypes ${ }^{10,12}$ (or only in very small numbers ${ }^{11}$ ), combined HNC with esophageal ${ }^{9,10,12}$ and gastric ${ }^{11}$ cancer into upper aero-digestive tract cancer (UADTC), upper digestive tract cancer (UTDC), or upper aerogastric tract (UAGT) cancers for analyses, or lacked information on smoking duration ${ }^{13,14}$, an important facet of smoking behavior. Our large prospective cohort study, with 415 HNC cases (131 OCC, 88 OHPC, and 193 LC) therefore adds significantly to the existing evidence-largely based on case-control studies-regarding the association between lifestyle factors and HNC risk.

Nevertheless, some questions remain unresolved. With regard to BMI, a low BMI $\left(<18.5 \mathrm{~kg} / \mathrm{m}^{2}\right)$ at baseline was inversely associated with HNC risk, whereas for BMI at age 20 , a positive rather than inverse association was found. In our study, we did not find indications for reverse causality, but residual confounding might play a role. We concluded that leanness itself is probably not a causal factor in the association with HNC, but we still do not know the exact mechanisms behind the possible association between $\mathrm{BMI}$ and HNC risk. This appears to be different than the relationship between $\mathrm{BMI}$ and many other cancers, where BMI clearly increases cancer risk. ${ }^{3}$ Furthermore, we did not find clear associations for vitamins and carotenoids other than vitamin $\mathrm{C}$ on HNC risk. In addition, we found different associations of vitamin supplements and vitamin intake through food on HNC risk. This could be a chance finding or be explained by the small sample size of supplement users, but it might also be that vitamins from supplements simply have different properties than those from whole foods (e.g., vitamin C from vegetables and fruits). 
In that view, there are potential-more systemic-mechanisms besides antioxidant properties for the effect of diet and other lifestyle factors on HNC risk which have been less described. First, diet might affect the body's acid-base balance by promoting either acidity or alkalinity, thereby possibly influencing tumor growth. ${ }^{24,25} \mathrm{~A}$ diet rich in vegetables-especially raw vegetables-and fruits may promote alkalinity, which may exert a beneficial effect on HNC risk. ${ }^{24,25}$ Furthermore, diet may influence the process of low-grade, chronic inflammation in the body, which is likely to be involved in cancer risk and development as well. ${ }^{26-31}$ Chronic inflammation has been widely studied and related to many diseases, including cancer and cardiovascular diseases. Circulating inflammation marker levels have been associated with lung cancer risk in a prospective study. ${ }^{32}$ In addition, poor oral hygiene may lead to chronic inflammation (such as periodontitis) as well, and may also be associated with an increased HNC risk. ${ }^{33}$ Poor oral hygiene-and possibly other lifestyle factors-might promote the oncogenesis of HNC through chronic inflammation by creating an inflammatory microenvironment that promotes tumor development. ${ }^{29}$ Therefore, when we look at the overall picture of dietary factors, smoking, alcohol consumption, oral hygiene, and perhaps also human papillomavirus (HPV), inflammation might even be a common mechanism by which all these factors may influence the risk of developing HNC.

Moreover, poor oral hygiene is also likely related to alcohol consumption and cigarette smoking (and the use of drugs), and might be related to diet. Literature regarding the possible relationship between diet and oral hygiene is scarce, but consumption of certain foods-amongst others vegetables and fruits-might positively influence oral hygiene, possibly as a result of mechanic oral cleaning. ${ }^{34,35}$ This might, depending on time of intake, especially be the case in smokers and alcohol drinkers.

To further elaborate on this, it might be promising to closer examine the relationship between dietary patterns (including smoking habits and alcohol consumption) and HNC risk, in addition to the association between food groups and food items on risk of HNC. In everyday practice, people do not just eat separate food items, but generally develop a certain dietary and lifestyle pattern which might have systemic effects (such as described above) on cancer risk. For example, people who eat more vegetables and fruits might have a generally healthier lifestyle, reflecting another dietary pattern than that of people who consume more animal and/or processed foods. ${ }^{36,37}$ So far, a dietary pattern with relatively high vegetable and fruit intake and low intake of animal and processed foods has been associated with reduced HNC risk. $^{36-38}$ Studying dietary patterns could, therefore, lead to a broader mechanistic view on the relationship between diet and HNC risk. 
Finally, in our study, we did not have data on HPV status, nowadays another established risk factor for $\mathrm{HNC}^{1}$ None of the prospective studies regarding alcohol, smoking, dietary factors and HNC included HPV data in their research either. Ideally, we would have included such data in our study to adjust for in our analyses in the best possible way, but at the start of our study in 1986, the influence of HPV was not fully known yet. Preferably, all diagnosed oropharyngeal cancer cases during follow-up would have been analyzed on HPV-positivity or -negativity, but gathering this information in our cohort would have been very difficult. For several reasons, however, we argued that the lack of HPV data does probably not significantly influence our findings (see paragraph 'Methodological considerations').

In addition, tumor development is classically divided into three stages: tumor initiation, tumor promotion, and tumor progression. As a carcinogen ${ }^{1}$, HPV is likely to be at least involved in tumor initiation. The influence of lifestyle factors might, in that case, subsequently play a role in tumor promotion and progression, by creating a tumor microenvironment that either promotes or inhibits tumor development. ${ }^{30}$ For that reason, we would like to speculate that diet may affect HPV-related HNC as well, in a way that HPV may initiate HNC, but nutrition and lifestyle habits may then promote and progress-or inhibit-tumor growth. In that view, our findings may be interesting for HPV-related HNC as well, even though we did not include HPV in our study; this warrants further research.

\section{Differences among head-neck cancer subtypes}

In our study, we found consistent differences in associations with risk of HNC subtypes. In general, the risk of OCC and OHPC seemed to be more influenced by alcohol and nutritional factors than LC. This was a consistent pattern (Table 7.1).

How can we explain this differential risk pattern? HNC subtypes are differently located in the head-neck area: OCC and OHPC are part of the aerodigestive tract, whereas LC is part of the respiratory tract. Therefore, specific anatomic subsites come directly in contact with different substances, including potentially HNC-related matters such as carcinogens (e.g., ethanol) and antioxidants (e.g., vitamin C). These substances may have an indirect, systemic effect (i.e., they become available in the body after being metabolized and subsequently act in numerous processes across the body, e.g., acetaldehyde, the main metabolite of ethanol), but they may also exert their effect on HNC carcinogenesis through direct exposure to tissue in the head-neck area. The differential risk among HNC subtypes may therefore be explained by the larynx having 
the least direct exposure to alcohol and food substances compared with the oral cavity and pharynx. ${ }^{4,6}$ In addition, the oral cavity seems to be the least susceptible to the effects of cigarette smoking. ${ }^{39,40}$ This leads us to another possible explanation for the HNC risk pattern regarding cigarette smoking, which could be the aerodynamics of respiratory flow in the upper airway. This flow changes from predominantly laminar in the oral cavity to predominantly turbulent in the larynx, which may result in the larynx and pharynx having a higher exposure to inhaled air-and thus to cigarette smokethan the oral cavity. Differences in associations among HNC subtypes could thus be explained by different exposures due to the location of the tumor.

Regarding selenium, strongest inverse associations were found for OHPC and LC, which in our study were the subtypes also strongest associated with cigarette smoking. Our findings might point to the possibility that, despite thorough adjustment for confounding by smoking, there might still be residual confounding. The fact that most associations were substantially stronger in age- and sex-adjusted analyses than in multivariable-adjusted analyses further implies this possibility. However, it may also indicate that the effect of selenium on HNC risk is associated with smoking. We did show that toenail selenium was inversely associated with HNC risk especially in current smokers, but without a statistically significant interaction, perhaps due to a lack of power. It might be biologically plausible that the effect of selenium on HNC risk is associated with smoking, since selenium is an antioxidant and counteracts the oxidative stress caused by smoking. ${ }^{3}$ This would, however, raise the question why we did not find a similar pattern among HNC subtypes with regard to vegetable and fruit consumption, and vitamin intake.

\section{Methodological considerations}

Our study has several important strengths, as well as some limitations, and there are interesting methodological points to consider.

Important strengths of our study are the prospective nature and duration and completeness of follow-up. This makes recall bias, reversed causation and loss to follow-up bias unlikely. Given our large number of cases, we were able to examine the effect of lifestyle factors not only on risk of HNC overall, but also on the risk of HNC subtypes. Furthermore, we had the ability to adjust for confounders extensively, which is vitally important since cigarette smoking and alcohol consumption are main risk factors for HNC. 
A possible limitation of our study is the single measurement of exposure data. Although validation and reproducibility studies showed good results ${ }^{41,42}$, the FFQ may have provided only moderately accurate estimates of food intake and other lifestyle habits. We believe the FFQ was given to participants in an age group with stable dietary habits at baseline, but it is nevertheless possible that participants changed their dietary habits since 1986. Although cigarette smoking and alcohol consumption were extensively addressed, it is also possible that participants changed their smoking habits and/or habitual alcohol intake at some point during follow-up. All this may have led to bias due to random (or non-differential) misclassification of exposure, possibly resulting in an underestimation of the effects of the studied factors on HNC risk. It may also have led to bias when adjusting for smoking as a confounder, but we did take this into account in our analyses by investigating and correcting for an interaction of smoking status with time if necessary (see Chapter 3).

We cannot exclude the presence of some residual confounding by smoking and/or alcohol, although we had detailed information about alcohol consumption and cigarette smoking and were therefore able to thoroughly adjust for these confounders. Residual confounding may also have occurred due to other potential confounders, such as physical activity and family history of HNC, but as we investigated many of these, we presume this to be limited.

Furthermore, although our study includes a large number of cases, a lack of power is a possible explanation for finding non-significant results for some associations and the tests for heterogeneity. In stratified analyses, we mostly did not find a statistically significant interaction with regard to cigarette smoking status or alcohol consumption. However, analyses included only few cases among never smokers and never drinkers, and there may have been a lack of power to detect a significant interaction. Finally, our categorization of alcohol consumption may have affected results of the (interaction) analyses, but we tested several options and used the best possible categorization for each analysis.

Our study lacks information on HPV infection, which has been associated with HNC risk. $^{1,43-45}$ HPV infection is associated with HNC risk ${ }^{43,46}$, but mainly with OHPC, in particular tonsil cancer and cancer of the base of the tongue. According to rates in our university medical center (personal communication of prof. dr. B. Kremer), only $25 \%$ of the diagnosed and treated oropharyngeal cancers between 1997 and 2003 were HPVpositive (all oropharyngeal cancer cases have been analyzed by p16-immunostaining and HPV16-specific fluorescence in situ hybridization (FISH), and-if FISH was negative-HPV-specific polymerase chain reaction). Moreover, the role of HPV in HNC carcinogenesis is mainly of importance in young HNC patients, and has increased since 
$1990^{45,47,48}$, whereas our participants were aged 55-69 years at baseline in 1986. For these reasons, we assume that the number of HPV-associated HNC cases in our cohort is low, and we expect potential bias due to possible misclassification to be very limited.

We were not able to include the use of drugs and oral hygiene in our study. As mentioned before, we speculate that oral hygiene may play a role in the development of HNC, especially OCC, and it might in that view be interesting to include this factor in future studies. ${ }^{33,49}$

Future studies might be able to address and elaborate on some of the possible limitations we had in our study, as will be described in the last paragraph.

\section{Implications for public health}

With regard to HNC, it has been estimated that up to half of these cancers are preventable by appropriate diets and associated lifestyle factors. ${ }^{3}$ As we wrote in Chapter 1, treatment decisions are complex, often with side effects resulting in significant morbidity with regard to basic functions such as speaking, swallowing, and breathing. Our results strengthen the evidence that lifestyle factors importantly influence HNC risk, and health guidelines may help support and encourage people to act accordingly.

Furthermore, the lifestyle factors that influence the risk of HNC and HNC subtypes also influence risk of other diseases, such as cardiovascular diseases (CVD) and other types of cancer. Cigarette smoking and alcohol consumption are known risk factors for CVD and many types of cancer. Dietary factors, like vegetable and fruit intake, importantly influence risk of other diseases as well. The same applies for overweight and obesity.

In addition to HNC, the ongoing WCRF systematic review of worldwide research ${ }^{50}$ shows that about a third of the most common cancers are preventable through a nutritious diet, maintaining a healthy weight and regular physical activity. The WCRF therefore set up Cancer Prevention Recommendations, which are the result of years of research in this field. Where applicable, our results support these recommendations (Table 7.2).

Ultimately, the goal of health guidelines is to decrease the burden of disease and mortality due to preventable causes. In that perspective, our results underline that prevention-by making healthy lifestyle choices-is a promising strategy in HNC. 
Table 7.2. WCRF Cancer Prevention Recommendations ${ }^{50}$

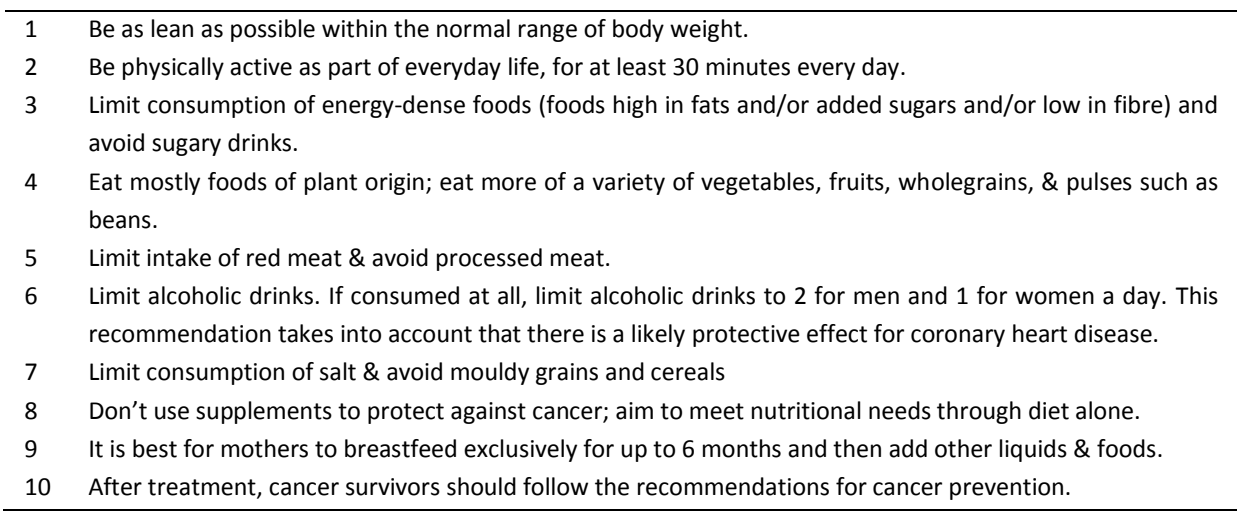

\section{Concluding remarks}

The general aim of this thesis was to study and further establish the existing evidence regarding the association between several lifestyle factors and the risk of developing HNC and HNC subtypes.

Our results confirm the principal role of alcohol consumption and cigarette smoking in HNC carcinogenesis. As we hypothesized, the risk of HNC was higher in participants with a low intake of vegetables and fruits and vitamin $\mathrm{C}$, and those with low levels of toenail selenium. No clear associations were, however, found for intake of vitamin E and carotenoids. In addition, the risk of HNC was lower in participants with a high BMI at study baseline, but not at age 20; we concluded that leanness itself is probably not a causal factor in the association with HNC. Associations of the studied lifestyle factors with HNC risk were not clearly modified by smoking and alcohol consumption in our study, although we expected otherwise. Finally, we did find a consistent pattern in differences in risk associations among the HNC subtypes OCC, OHPC, and LC: LC risk seemed to be more smoking related, whereas alcohol and nutrition appeared to have a stronger influence on risk of OCC.

In conclusion, lifestyle factors, including nutrition, significantly influence HNC risk. As prospective data are scarce, this cohort study considerably contributes to establish in which extent lifestyle factors are associated with risk of HNC overall and especially HNC subtypes. Nevertheless, there are specific items that seem to be important to address in future studies regarding the etiology of HNC. 


\section{Recommendations for future research}

Future research is warranted for further clarifications of the possible mechanisms involved regarding lifestyle factors and HNC risk, and to confirm our results, especially associations between vitamin and carotenoid intake, toenail selenium status, BMI and $\mathrm{HNC}$ risk. We found a differential risk between toenail selenium and HNC risk in men and women, but it is unclear what reasons there might be behind this possible difference. It would therefore be interesting to further study this association and the possible mechanisms behind it.

Since our results indicate clear association differences among the most common HNC subtypes, in future research these tumor groups would ideally be split up, as there likely are different mechanistic pathways involved. We especially recommend studying OCC and LC separately, if possible. In our study, OHPC risk mostly resembled OCC risk, but it requires more research to put this tumor group in perspective, perhaps more correctly, and perhaps even further split up into oropharyngeal and hypopharyngeal cancer. It would, however, require large case-numbers of OCC, OHPC, and LC to further study and clarify these associations pattern we found.

We also recommend including HPV-status in future studies regarding HNC etiology. As HPV-related HNC incidence has been on the rise since 1990, we believe that future prospective studies need to incorporate HPV in (the set-up of) their study. Although still a minority of HNC is HPV-related, it is important to include HPV in future studies in order to obtain the most comprehensive view on HNC risk.

We did not find clear interaction patterns between alcohol consumption, cigarette smoking and other lifestyle factors, but that might have to do with a lack of power, even though our study is one of the largest prospective studies so far. To examine these interactions therefore warrants further study, in particular with larger casenumbers and thus more study power, or possibly in a pooled analysis of prospective studies. It might, in that view, also be interesting to examine the RRs of HNC for smokers among non-drinkers and for drinkers among non-smokers with large casenumbers. However, as case numbers for these subgroups are generally very small, this item will probably be difficult to address.

Finally, there are other interesting factors-though outside the scope of this thesis - to include in future research to broaden the proverbial 'HNC horizon'. First, it might be interesting to not only further investigate dietary factors per se, but also in terms of dietary patterns, as we described earlier. Furthermore, psychosocial factors ${ }^{51}$ have been studied in relationship to cancer risk, but this association remains unclear and controversial. According to the US National Cancer Institute ${ }^{51}$, the body responds 
to physical, mental, or emotional stress by releasing stress hormones that increase blood pressure and raise blood sugar levels. Research has shown that people who experience chronic stress can have digestive problems, fertility problems, a weakened immune system, and are also more prone to viral infections. Given these physical responses, chronic stress may also lead to the previously described process of chronic inflammation. It might therefore be interesting to explore whether psychosocial health may contribute to $\mathrm{HNC}$ risk, and-just to speculate in this thesis-in the future perhaps be able to model HNC risk in an integral biopsychosocial model. Also, given the importance of lifestyle factors in HNC risk, gene-environment interactions might also be of interest to explore with respect to HNC risk, with genes relevant in HNC etiology. Lastly, it might also be interesting to study the influence of lifestyle factors after HNC diagnosis with regard to HNC survivorship, and find out if it might literally never be too late for lifestyle changes.

\section{References}

1 IARC. A Review of Human Carcinogens: Biological Agents. Vol. 100B International Agency for Research on Cancer, 2012.

2 IARC. Monographs on the evaluation of carcinogenic risks to humans: personal habits and indoor combustions. International Agency for Research on Cancer, 2012.

3 World Cancer Research Fund \& American Institute for Cancer Research. Food, Nutrition, Physical Activity and the Prevention of Cancer: a Global Perspective. American Institute for Cancer Research, 2007.

4 Boffetta, P. \& Hashibe, M. Alcohol and cancer. Lancet Oncol 7, 149-156 (2006).

5 IARC. Monographs on the evaluation of carcinogenic risks to humans: tobacco smoke and involuntary smoking. International Agency for Research on Cancer, 2004.

6 Purdue, M. P. et al. Type of alcoholic beverage and risk of head and neck cancer--a pooled analysis within the INHANCE Consortium. Am J Epidemiol 169, 132-142 (2009).

7 International Agency for Research on Cancer \& World Health Organization. IARC Handbooks of Cancer Prevention: Fruit and Vegetables. IARC Press, 2003.

8 Rayman, M. P. Selenium in cancer prevention: a review of the evidence and mechanism of action. The Proceedings of the Nutrition Society 64, 527-542 (2005).

9 Boeing, H. et al. Intake of fruits and vegetables and risk of cancer of the upper aero-digestive tract: the prospective EPIC-study. Cancer Causes Control 17, 957-969 (2006).

10 Chyou, P. H., Nomura, A. M. \& Stemmermann, G. N. Diet, alcohol, smoking and cancer of the upper aerodigestive tract: a prospective study among Hawaii Japanese men. Int J Cancer 60, 616-621 (1995).

11 Kasum, C. M., Jacobs, D. R., Jr., Nicodemus, K. \& Folsom, A. R. Dietary risk factors for upper aerodigestive tract cancers. Int J Cancer 99, 267-272 (2002).

12 Kjaerheim, K., Gaard, M. \& Andersen, A. The role of alcohol, tobacco, and dietary factors in upper aerogastric tract cancers: a prospective study of 10,900 Norwegian men. Cancer Causes Control 9, 99-108 (1998).

13 Freedman, N. D., Abnet, C. C., Leitzmann, M. F., Hollenbeck, A. R. \& Schatzkin, A. Prospective investigation of the cigarette smoking-head and neck cancer association by sex. Cancer 110, 1593-1601 (2007).

14 Freedman, N. D. et al. Fruit and vegetable intake and head and neck cancer risk in a large United States prospective cohort study. Int J Cancer 122, 2330-2336 (2008).

15 Freedman, N. D., Schatzkin, A., Leitzmann, M. F., Hollenbeck, A. R. \& Abnet, C. C. Alcohol and head and neck cancer risk in a prospective study. Br J Cancer 96, 1469-1474 (2007). 
Boeing, $\mathrm{H}$. Alcohol and risk of cancer of the upper gastrointestinal tract: first analysis of the EPIC data. IARC Sci Publ 156, 151-154 (2002).

17 Zheng, W. et al. Retinol, antioxidant vitamins, and cancers of the upper digestive tract in a prospective cohort study of postmenopausal women. Am J Epidemiol 142, 955-960 (1995).

18 Rogers, M. A. et al. A case-control study of oral cancer and pre-diagnostic concentrations of selenium and zinc in nail tissue. Int J Cancer 48, 182-188 (1991).

19 Zheng, W. et al. Serum micronutrients and the subsequent risk of oral and pharyngeal cancer. Cancer Res 53, 795-798 (1993).

20 Knekt, P. et al. Serum micronutrients and risk of cancers of low incidence in Finland. Am J Epidemiol 134, 356-361 (1991).

21 Hashibe, M. et al. Tobacco, alcohol, body mass index, physical activity, and the risk of head and neck cancer in the prostate, lung, colorectal, and ovarian (PLCO) cohort. Head Neck 35, 914-922 (2013).

22 Gaudet, M. M. et al. Prospective studies of body mass index with head and neck cancer incidence and mortality. Cancer Epidemiol Biomarkers Prev 21, 497-503 (2012).

23 Etemadi, A. et al. A prospective cohort study of body size and risk of head and neck cancers in the NIH-AARP diet and health study. Cancer Epidemiol Biomarkers Prev 23, 2422-2429 (2014).

24 Robey, I. F. Examining the relationship between diet-induced acidosis and cancer. Nutr Metab (Lond) 9, 72 (2012).

25 Welch, A. A., Mulligan, A., Bingham, S. A. \& Khaw, K. T. Urine pH is an indicator of dietary acid-base load, fruit and vegetables and meat intakes: results from the European Prospective Investigation into Cancer and Nutrition (EPIC)Norfolk population study. Br J Nutr 99, 1335-1343 (2008).

26 Cao, Y., DePinho, R. A., Ernst, M. \& Vousden, K. Cancer research: past, present and future. Nat Rev Cancer 11, 749-754 (2011).

27 Coussens, L. M. \& Werb, Z. Inflammation and cancer. Nature 420, 860-867 (2002).

28 Julia, C. et al. Dietary patterns and risk of elevated C-reactive protein concentrations 12 years later. Br J Nutr 110, 747754 (2013).

29 Mantovani, A., Allavena, P., Sica, A. \& Balkwill, F. Cancer-related inflammation. Nature 454, 436-444 (2008).

30 Hanahan, D. \& Weinberg, R. A. Hallmarks of cancer: the next generation. Cell 144, 646-674 (2011).

31 Roxburgh, C. S. \& McMillan, D. C. Cancer and systemic inflammation: treat the tumour and treat the host. Br J Cancer 110, 1409-1412 (2014).

32 Shiels, M. S. et al. Circulating inflammation markers and prospective risk for lung cancer. J Nat/ Cancer Inst 105, 18711880 (2013).

33 Chang, J. S. et al. Investigating the association between oral hygiene and head and neck cancer. Oral oncology 49, 1010-1017 (2013).

34 Michaud, D. S. et al. Lifestyle, dietary factors, and antibody levels to oral bacteria in cancer-free participants of a European cohort study. Cancer Causes Control 24, 1901-1909 (2013).

35 Staufenbiel, I., Weinspach, K., Forster, G., Geurtsen, W. \& Gunay, H. Periodontal conditions in vegetarians: a clinical study. Eur J Clin Nutr 67, 836-840 (2013).

36 Bradshaw, P. T. et al. Associations between dietary patterns and head and neck cancer: the Carolina head and neck cancer epidemiology study. Am J Epidemiol 175, 1225-1233 (2012).

37 Bravi, F. et al. Dietary patterns and upper aerodigestive tract cancers: an overview and review. Ann Oncol 23, 30243039 (2012).

38 Chuang, S. C. et al. Diet and the risk of head and neck cancer: a pooled analysis in the INHANCE consortium. Cancer Causes Control 23, 69-88 (2012).

39 Hashibe, M. et al. Alcohol drinking in never users of tobacco, cigarette smoking in never drinkers, and the risk of head and neck cancer: pooled analysis in the International Head and Neck Cancer Epidemiology Consortium. J Natl Cancer Inst 99, 777-789 (2007).

40 Zeka, A., Gore, R. \& Kriebel, D. Effects of alcohol and tobacco on aerodigestive cancer risks: a meta-regression analysis. Cancer Causes Control 14, 897-906 (2003).

41 Goldbohm, R. A. et al. Validation of a dietary questionnaire used in a large-scale prospective cohort study on diet and cancer. Eur J Clin Nutr 48, 253-265 (1994).

42 Goldbohm, R. A. et al. Reproducibility of a food frequency questionnaire and stability of dietary habits determined from five annually repeated measurements. Eur J Clin Nutr 49, 420-429 (1995).

43 Hobbs, C. G. et al. Human papillomavirus and head and neck cancer: a systematic review and meta-analysis. Clin Otolaryngol 31, 259-266 (2006). 
44 Marur, S., D'Souza, G., Westra, W. H. \& Forastiere, A. A. HPV-associated head and neck cancer: a virus-related cancer epidemic. Lancet Oncol 11, 781-789 (2010).

45 Mehanna, H. et al. Prevalence of human papillomavirus in oropharyngeal and nonoropharyngeal head and neck cancer--systematic review and meta-analysis of trends by time and region. Head Neck 35, 747-755 (2013).

46 D'Souza, G. et al. Case-control study of human papillomavirus and oropharyngeal cancer. N Engl J Med 356, 1944-1956 (2007).

47 Curado, M. P. \& Hashibe, M. Recent changes in the epidemiology of head and neck cancer. Curr Opin Oncol 21, 194200 (2009).

48 Marur, S. \& Forastiere, A. A. Head and neck cancer: changing epidemiology, diagnosis, and treatment. Mayo Clin Proc 83, 489-501 (2008).

49 Tsai, S. T. et al. The interplay between alcohol consumption, oral hygiene, ALDH2 and ADH1B in the risk of head and neck cancer. Int J Cancer 135, 2424-2436 (2014).

50 World Cancer Research Fund. Our Cancer Prevention Recommendations. London, UK: World Cancer Research Fund International. Available from: http://www.wcrf.org/int/research-we-fund/our-cancer-prevention-recommendations, accessed 30/10/2015.

51 National Cancer Institute. Psychological Stress and Cancer. USA: National Cancer Institute. Available from: http://www.cancer.gov/about-cancer/coping/feelings/stress-fact-sheet, accessed 26/11/2015. 
Summary 
Head-neck cancer (HNC) is the seventh most common type of cancer in the world and Europe. Cancers located in the oral cavity, pharynx, and larynx are the most frequent HNC subtypes, and they largely share the same risk factors (Chapter 1). Treatment decisions are complex, with considerable early and late side effects. In addition, survival rates are variable and average around 50-60 per cent at 5 years, depending on the type or anatomic sublocalization of HNC.

It has been estimated that up to half of HNC cases are preventable by lifestyle factors and appropriate diets. Especially since treatment options are limited and survival rates poor, the focus on prevention of HNC-by lifestyle-is of great importance. Evidence with regard to lifestyle and dietary factors and HNC risk, in particular risk of HNC subtypes, is limited and mainly based on case-control studies; prospective data are scarce. Therefore, the general aim of this thesis was to study the association between several lifestyle factors and the risk of developing HNC and HNC subtypes-i.e., oral cavity cancer (OCC), oro-/hypopharyngeal cancer (OHPC), and laryngeal cancer (LC)-in a large prospective cohort study.

We investigated the associations between alcohol consumption, cigarette smoking, consumption of vegetables and fruits, vitamin and carotenoid intake, toenail selenium status, and body mass index (BMI) and risk of HNC and HNC subtypes within the Netherlands Cohort Study on diet and cancer (NLCS). The NLCS was initiated in September 1986 and includes 120,852 participants, 58,279 men and 62,573 women, aged 55-69 years from 204 Dutch municipal population registries. At baseline, all participants completed a self-administered questionnaire on dietary habits and other cancer risk factors, such as smoking, alcohol consumption, and anthropometry. The 11page questionnaire included a 150-item food frequency questionnaire that focused on habitual food consumption during the year preceding the start of the study. In addition, about $75 \%$ of the participants collected toenail clippings.

For the NLCS, the case-cohort design was used for efficiency in data processing, follow-up and analyses. Cases were derived from the entire cohort, whereas the number of person-years at risk for the entire cohort was estimated using a subcohort of 5,000 people, randomly sampled from the total cohort at baseline and followed up for vital status information. The entire cohort is being monitored for cancer incidence by annual record linkage to the Netherlands Cancer Registry, and the nationwide network and registry of histo- and cytopathology in the Netherlands. After 20.3 years of follow-up, 415 incident HNC (131 OCC, 88 OHPC, 3 oral cavity/pharynx unspecified or overlapping, and 193 LC) cases were available for analysis.

In Chapter 2, we studied the association between alcohol consumption, cigarette smoking and HNC risk. Alcohol consumption and cigarette smoking were strongly, 
independently associated with an increased HNC risk. In addition, we found a positive, multiplicative interaction between both factors regarding HNC risk. The strength of these associations, however, differed among subtypes; OCC was most strongly associated with alcohol consumption but most weakly with cigarette smoking, whereas LC was not statistically significantly associated with alcohol consumption.

Next, we found that total vegetable and fruit consumption were related to a decreased risk of HNC overall and all HNC subtypes (Chapter 3 ), with the strongest inverse associations for OCC. Total vegetable consumption was inversely associated with risk of HNC overall as well; associations with HNC subtypes were also inverse and were largely similar for OCC, OHPC, and LC. Total fruit consumption was associated with reduced risks of $\mathrm{HNC}$ overall and all subtypes; the strongest associations were with OCC. Associations between vegetable and fruit consumption and HNC risk were not clearly modified by cigarette smoking status or alcohol consumption.

In Chapter 4, we examined associations of vitamin and carotenoid intake with HNC risk. Vitamin $C$ intake through diet was significantly associated with a decreased risk of HNC overall, OCC and OHPC. No clear associations were found for other nutrients and vitamin supplementation; however, most point estimates showed possible protective effects. Furthermore, the association between vitamin $E$ intake and the risk of HNC overall was modified by alcohol consumption, with lower risks in alcohol abstainers.

In Chapter 5, we investigated selenium, a trace element present in food which is likely to be involved in cancer risk. A low toenail selenium level was related to an increased HNC risk in our study. Among HNC subtypes, this inverse association was strongest for OHPC and LC. The association between toenail selenium and risk of HNC overall was considerably stronger among men than women. Although there was no clear effect modification by cigarette smoking or alcohol consumption in our study, the inverse association we found was most explicit among current smokers.

Lastly, we found an inverse association between BMI at baseline and HNC risk (Chapter 6): being underweight (BMI $<18.5 \mathrm{~kg} / \mathrm{m}^{2}$ ) at baseline was related to an increased HNC risk. Among HNC subtypes, this association was strongest for OCC and OHPC. For BMI at age 20 , on the other hand, we found a positive rather than inverse association. The association between change in BMI since the age of 20 years and HNC risk appeared to be inverse again. There was effect modification by alcohol consumption in our study, with the lowest risks of HNC overall for BMI at baseline, BMI at age 20 , and change in BMI in non-drinkers.

We generally discuss our findings and put them into perspective in Chapter 7. Our results strengthen the evidence that lifestyle factors importantly influence HNC risk. Alcohol consumption and cigarette smoking play a principal role in HNC carcinogenesis. 
Furthermore, a low intake of vegetables, fruits and vitamin C were associated with an increased HNC risk, as well as low levels of toenail selenium. Intake of a vitamin C supplement, vitamin $\mathrm{E}$ and carotenoids, on the other hand, do not seem to influence $\mathrm{HNC}$ risk. Regarding BMI, we concluded that leanness itself is probably not a causal factor in the association with HNC; HNC risk was lower in participants with a high BMI at study baseline, but not at age 20. Associations of the studied lifestyle factors with $\mathrm{HNC}$ risk were not clearly modified by smoking and alcohol consumption.

Regarding HNC subtypes, we found consistent differences in risk associations. In general, the risk of OCC and OHPC seemed to be more influenced by alcohol and nutritional factors than LC. Therefore, in future research regarding the etiology of HNC, these tumor groups would ideally be split up. Future research is also warranted for further clarifications of the possible mechanisms involved regarding lifestyle factors and HNC risk, and to confirm our results. Furthermore, we recommend including HPV status in future studies. In conclusion, the results described in this thesis underline that prevention-by making healthy lifestyle choices-is a promising strategy in HNC. 
Samenvatting 
Hoofd-halskanker (HHK) is wereldwijd en in Europa de zevende meest voorkomende vorm van kanker. Kanker van de mondholte, keelholte en het strottenhoofd zijn de meest voorkomende subtypen van HHK en kennen grotendeels dezelfde risicofactoren (Hoofdstuk 1). De vraag hoe HHK patiënten het best behandeld kunnen worden is vaak complex, omdat behandeling nadelige gevolgen kan hebben voor diverse lichaamsfuncties, op zowel korte als lange termijn. De gemiddeld 5-jaarsoverleving voor HHK is $50-60 \%$; afhankelijk van het type of anatomische sublokalisatie van HHK verschillen de overlevingskansen aanzienlijk.

Er wordt geschat dat de helft van alle gevallen van HHK voorkómen kan worden door leefstijlfactoren, waaronder een gezond voedingspatroon. Zeker omdat behandelingsopties beperkt en overlevingskansen gering zijn, is aandacht voor de preventie van HHK door leefstijl daarom van groot belang. Het bewijs voor het verband tussen leefstijl, voedingsfactoren en het risico op HHK, met name het risico op HHK subtypen, is echter beperkt en voornamelijk gebaseerd op case-control studies; prospectieve gegevens zijn schaars. Het doel van dit proefschrift is dan ook het verband tussen verschillende leefstijlfactoren en het risico op HHK en HHK subtypeni.e., kanker van de mondholte (MK), keelholte (de oro- en hypofarynx: OHK) en het strottenhoofd (de larynx: LK) - te bestuderen in een grote prospectieve cohortstudie.

We hebben het verband tussen alcoholgebruik, roken, de consumptie van groente en fruit, de inname van vitaminen en carotenoïden, seleniumwaarden in teennagels, body mass index (BMI) en het risico op HHK en HHK subtypen onderzocht binnen de Nederlandse Cohort Studie naar voeding en kanker (NLCS). De NLCS werd opgericht in september 1986 en bestaat uit 120.852 deelnemers, 58.279 mannen en 62.573 vrouwen, in de leeftijd van 55-69 jaar uit 204 gemeentelijke bevolkingsregisters. Bij aanvang van de studie hebben alle deelnemers een vragenlijst over voedingsgewoonten en andere kankerrisicofactoren, zoals roken, alcoholgebruik en lichaamsgewicht, ingevuld. Deze 11 pagina's tellende vragenlijst bestond onder meer uit een voedingsvragenlijst van 150 items, gericht op de gebruikelijke consumptie van voedingsmiddelen in het jaar voorafgaand aan de start van de studie. Daarnaast heeft ongeveer $75 \%$ van de deelnemers een teennagelmonster aangeleverd.

In de NLCS gebruiken we voor de analyses het zogeheten case-cohort design vanwege efficiëntie in dataverwerking en follow-up. Cases zijn afkomstig uit het gehele cohort, terwijl het aantal persoonsjaren 'at risk' voor het hele cohort wordt geschat aan de hand van een subcohort. Dit subcohort, waarvan ook de vitale status wordt opgevolgd, is een willekeurige subgroep van 5.000 deelnemers uit het totale cohort bij baseline. De incidentie van kanker wordt in het gehele cohort opgevolgd door middel van jaarlijkse koppeling met de Nederlandse Kankerregistratie en het landelijk netwerk 
en register van histo- en cytopathologie (PALGA). Na ruim 20 jaar follow-up waren 415 incidente gevallen van HHK (waarvan $131 \mathrm{MK}, 88 \mathrm{OHK}, 3$ mond-/keelholte niet nader gespecificeerd of overlappend en 193 LK) beschikbaar voor analyse.

In Hoofdstuk 2 hebben we het verband tussen alcoholgebruik, roken en het risico op HHK bestudeerd. Zowel alcoholgebruik als roken waren sterk en onafhankelijk van elkaar geassocieerd met een verhoogd risico op HHK. Daarnaast vonden we een positieve, multiplicatieve interactie tussen beide factoren met betrekking tot HHK risico. De sterkte van deze associaties verschilde echter tussen subtypen; MK was het sterkst geassocieerd met de consumptie van alcohol, maar het zwakst met het roken van sigaretten, terwijl LK niet statistisch significant geassocieerd was met alcoholgebruik.

De totale consumptie van groente en fruit was gerelateerd aan een verlaagd risico op HHK in het algemeen en alle HHK subtypen (Hoofdstuk 3), met de sterkste inverse verbanden voor MK. Totale groenteconsumptie was eveneens invers geassocieerd met HHK risico; ook verbanden met HHK subtypen waren invers en grotendeels vergelijkbaar voor MK, OHK en LK. Totale fruitconsumptie was geassocieerd met een verminderd risico op HHK in het algemeen en alle subtypen, met de sterkste verbanden voor MK. Associaties tussen groente- en fruitconsumptie en HHK risico veranderden niet duidelijk in verschillende categorieën van roken of alcoholgebruik.

In Hoofdstuk 4 onderzochten we het verband tussen de inname van vitaminen en carotenoïden en het risico op HHK. Inname van vitamine C via de voeding was significant geassocieerd met een verlaagd risico op HHK in het algemeen, MK en OHK. We vonden geen duidelijke associaties met HHK risico voor andere vitaminen en carotenoïden dan wel het gebruik van vitaminesupplementen, hoewel de meeste puntschattingen mogelijk beschermende effecten lieten zien. De relatie tussen vitamine $\mathrm{E}$ inname en het risico op HHK werd gemodificeerd door alcoholgebruik, met lagere risico's voor alcoholonthouders.

In Hoofdstuk 5 onderzochten we selenium, een sporenelement in de voeding dat waarschijnlijk betrokken is bij het risico op kanker. In onze studie was een laag seleniumgehalte in teennagels geassocieerd met een verhoogd risico op HHK. Onder HHK subtypen was dit inverse verband het sterkst voor OHK en LK. Het verband tussen teennagelselenium en $\mathrm{HHK}$ risico was aanzienlijk sterker onder mannen dan onder vrouwen. Hoewel onze studie geen duidelijke effect modificatie door het roken van sigaretten of het gebruik van alcohol liet zien, was de inverse associatie die we gevonden hebben wel het meest expliciet onder huidige rokers.

Ten slotte hebben we een invers verband tussen BMI bij aanvang van de studie ('BMI bij baseline') en het risico op HHK gevonden (Hoofdstuk 6): het hebben van 
ondergewicht (BMI $<18.5 \mathrm{~kg} / \mathrm{m}^{2}$ ) bij baseline was gerelateerd aan een verhoogd $\mathrm{HHK}$ risico. Onder HHK subtypen was dit inverse verband het sterkst voor MK en OHK. Voor BMI op 20-jarige leeftijd vonden we daarentegen juist een positief verband. De associatie tussen verandering in BMI sinds de leeftijd van 20 jaar en HHK risico leek weer invers te zijn. Er was effect modificatie door alcoholgebruik in onze studie, met de laagste risico's op HHK in het algemeen voor BMI bij baseline, BMI op 20-jarige leeftijd en verandering in BMI onder niet-drinkers.

In Hoofdstuk 7 hebben we onze bevindingen in het algemeen besproken; daarnaast hebben we geprobeerd deze in perspectief te plaatsen. Onze resultaten versterken het bewijs dat leefstijlfactoren in belangrijke mate van invloed zijn op het risico HHK te ontwikkelen. Alcoholgebruik en het roken van sigaretten spelen daarin een hoofdrol. Verder zijn een lage inname van groente, fruit en vitamine $C$, evenals een lage teennagelseleniumwaarde, geassocieerd met een verhoogd risico op HHK. Het gebruik van een vitamine $C$ supplement, evenals de inname van vitamine $E$ en carotenoïden, lijkt het risico op HHK daarentegen niet te beïnvloeden. Ten aanzien van BMI hebben we geconcludeerd dat het hebben van een laag lichaamsgewicht (BMI $<18.5 \mathrm{~kg} / \mathrm{m}^{2}$ ) op zichzelf waarschijnlijk niet een oorzakelijke factor is in de associatie met HHK. Het risico op HHK was weliswaar lager onder deelnemers met een hoge BMI bij aanvang van de studie, maar was níet lager onder deelnemers met een hoge BMI op 20-jarige leeftijd. Associaties tussen de onderzochte leefstijlfactoren en het risico op HHK werden niet duidelijk gemodificeerd door roken en alcoholgebruik.

Met betrekking tot HHK subtypen hebben we consistente verschillen in risico associaties gevonden. In het algemeen lijkt het risico op MK en OHK meer beïnvloed te worden door alcohol en voeding dan het risico op LK. Mede op basis van onze studie zouden we daarom willen aanbevelen om bij vervolgonderzoek gericht op de etiologie van HHK deze tumorgroepen te splitsen. Daarnaast is toekomstig onderzoek belangrijk voor verdere verheldering van de mogelijke mechanismen die betrokken zijn bij het verband tussen leefstijlfactoren en het risico op HHK, alsook om onze studieresultaten te bevestigen. Verder raden we het meenemen van HPV status in toekomstige studies aan. We kunnen concluderen dat de in dit proefschrift beschreven resultaten onderstrepen dat het maken van gezonde leefstijlkeuzes een veelbelovende strategie ter preventie van HHK is. 


\section{Valorization}


By law, Dutch universities have three main tasks: to educate at an academic level, to conduct scholarly research, and to ensure that research findings impact society. Valorization is the term that governmental and university policymakers use to denote this process of 'translating academic wisdom to societal benefit'. This chapter outlines the valorization potential of the research findings presented in this thesis.

\section{From scientific to societal value}

Knowledge valorization involves the process of creating societal and/or economic value from scientific knowledge. How could this dissertation's key finding - that lifestyle factors importantly influence HNC risk-have a societal impact that goes beyond well-cited publications?

It has been estimated that up to half of HNC cases are preventable by appropriate diets and associated lifestyle factors. ${ }^{1}$ The results described in this thesis underline that prevention-by making healthy lifestyle choices-is a promising strategy in HNC. Alcohol consumption and cigarette smoking are main risk factors for HNC and strongly increase HNC risk. Furthermore, we clearly showed that other lifestyle factors significantly influence HNC risk as well. Vegetable and fruit consumption, as well as vitamin $\mathrm{C}$ intake, were significantly associated with a decreased HNC risk. Finally, a low toenail selenium status was associated to an increased HNC risk, as well as being underweight (BMI $<18.5 \mathrm{~kg} / \mathrm{m}^{2}$ ) at baseline. In conclusion, lifestyle factors, including nutrition, significantly influence HNC risk. Especially since treatment options are limited and survival rates poor, the focus on prevention of HNC-by lifestyle-is of great importance.

Furthermore, the lifestyle factors that influence HNC risk also influence risk of other diseases, such as cardiovascular diseases (CVD) and other cancer types. The World Cancer Research Fund systematic review of worldwide research ${ }^{2}$ shows that not only many HNC cases, but also about a third of the most common cancers are preventable through diet, a healthy weight, and physical activity. Cigarette smoking and alcohol consumption are known risk factors for CVD and many types of cancer. Dietary factors, like vegetable and fruit intake, importantly influence risk of other diseases as well. The same applies for overweight and obesity.

In view of the above, our findings may have an impact in several ways. We will describe what merit our results might have for public health, both nationally and internationally; for health care, in general and for the individual; and finally for education and future research regarding lifestyle factors and HNC risk. 


\section{Translating research into (daily) practice: dietary recommendations?}

Together with prior and future research findings, our findings could jointly result in useful outcomes such as dietary recommendations. Our results strengthen the evidence that lifestyle factors importantly influence HNC risk, and health guidelines may help support and encourage people to act accordingly.

The Cancer Prevention Recommendations set up by the World Cancer Research Fund (WCRF) ${ }^{2}$ are an example of such guidelines (Chapter 7, Table 7.2, and Figure 1 below). These recommendations are the result of years of ongoing research, known as the WCRF Continuous Update Project (CUP) ${ }^{3}$. As part of the CUP, global scientific research-randomized controlled trials and cohort studies-on how diet, physical activity, and weight affect cancer risk and survival is being systematically reviewed. Among experts, the WCRF CUP it is a trusted, authoritative scientific resource, which underpins current guidelines and policy for cancer prevention. ${ }^{3}$

Our findings directly contribute to the CUP with regard to HNC, thereby proving their value from scientific results to practical recommendations. A review of the Cancer Prevention Recommendations is expected in 2017; where applicable, our results support the current recommendations. The American Institute for Cancer Research $(A I C R)^{4}$ is part of the WCRF and thus disseminates the same recommendations for cancer prevention. These recommendations enable everyone, from policy makers to members of the public, to have access to the most up-to-date information on how to minimize the risk of developing cancer.

Eventually, our findings may also contribute to the WCRF NOURISHING framework ${ }^{5}$. This framework helps policymakers worldwide to identify where action is needed to promote healthy diets and to reduce obesity and other non-communicable diseases, including cancer. As a result, many different policy actions have already been taken, a perfect example of valorization. Key domains are food environment (NOURIS), food system (H), and behavioral change (ING). Examples of areas where governments need to take action are to offer healthy foods and set standards in public institutions and other specific settings; to set incentives and rules to create a healthy retail and food service environment; to harness food supply chain \& actions across sectors to ensure coherence with health; to inform people about food \& nutrition through public awareness; to give nutrition advice and counselling in health care settings; to give nutrition education and skills. ${ }^{5}$ 


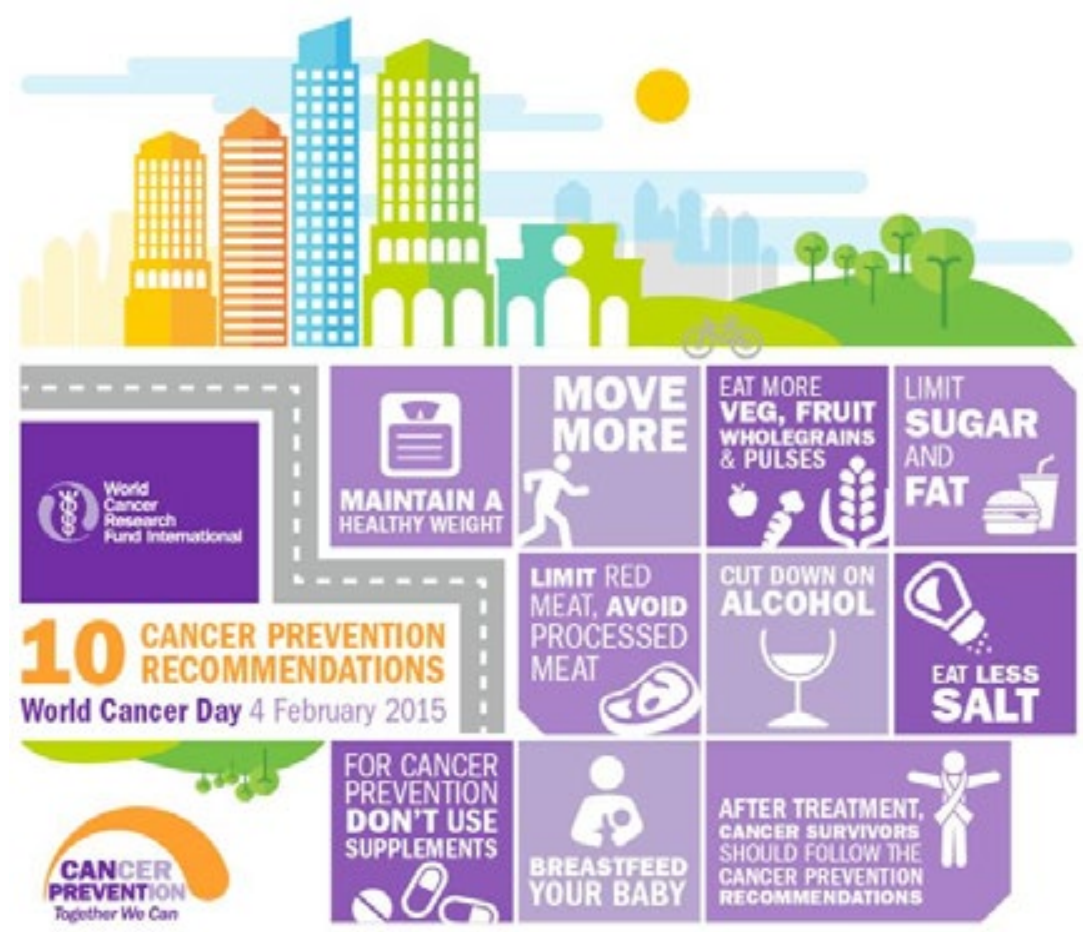

Figure 1. Infographic launched by $\mathrm{WCRF}^{2}$ for World Cancer Day: 'Empowering people to make healthy choices'

In addition to the WCRF and AICR, other public health organizations set up dietary recommendations to lower disease-including cancer-risk as well, based on sound scientific results which in the future might include ours. The World Health Organization (WHO) acts globally to help people eating healthier and governments enabling people to do so. ${ }^{6}$ The Health Council of the Netherlands (Gezondheidsraad) ${ }^{7}$ advises the Dutch authorities on health recommendations. Based on dietary guidelines of the Health Council $^{8}$, the Netherlands Nutrition Centre ${ }^{9}$ (NNC; Voedingscentrum) recently released their latest version of the so-called Food Pyramid, in Dutch better known as 'De Schijf van Vijf ${ }^{10}$ (Figure 2). The NNC states that healthy food is an essential part of a healthy lifestyle, and that healthy eating habits are often the best remedy for many of today's most common health problems as well as to prevent chronic diseases. The NNC seeks to explain the relevance of scientific knowledge and translate it into understandable and practical dietary guidelines. Lastly, the NNC puts the issue of healthier and more sustainable nutrition on the agendas of the food industry and consumers, as well as in politics and the media. ${ }^{9}$ 
The Food Pyramid is a practical information tool used by the NNC in order to encourage consumers to develop and maintain healthy eating habits, and to prevent obesity. Scientific results are thus being translated into a tool for daily practice. ${ }^{9,10}$ Key messages of the Food Pyramid are to eat lots of vegetables, fruits, and wholemeal products; less meat, and more legumes, nuts, eggs, or tofu instead; and to limit consumption of processed foods, which generally contain a lot of sugar, salt and unhealthy fats. ${ }^{9,10}$ Where applicable, the current recommendations of the Food Pyramid are in concordance with our study results.

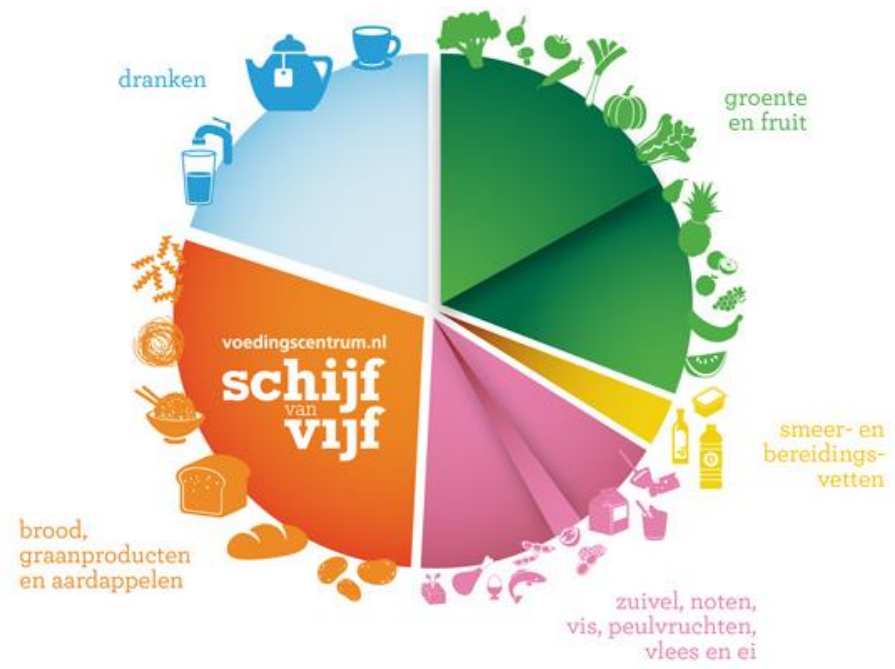

Figure 2. The Food Pyramid, released March $2016^{10}$

\section{Other possible implications for public health and healthcare}

In addition to a possible contribution to health recommendations and dietary guidelines, our results have been considered relevant and drawn attention in various ways. We communicated our study findings to various audiences, with possible implications for public health, healthcare, and future research.

In 2012, 2013 and 2015, our results were shown at the Dutch General Meeting for Otorhinolaryngology, with an audience consisting mostly of clinicians. In 2013, our study results were presented to a public of largely clinicians as well at the 4th World Congress of the International Academy of Oral Oncology in Greece. Furthermore, our study was one of the topics at the Rank Prize Funds mini-symposium on Alcohol and Nutrition in the United Kingdom in 2011. These Funds have as their objectives the advancement and promotion for the public benefit of knowledge, education and 
learning. Finally, our study findings have been presented at the Dutch Epidemiology Meeting in 2012. An invitation to speak for dentists, general practitioners and other clinicians at a conference entitled 'Nutrition-healthy living!' in November 2016 has been accepted.

Furthermore, our findings were highlighted in the Dutch media. In 2014, our results concerning alcohol consumption, cigarette smoking and HNC risk were published in Metro, a Dutch newspaper. ${ }^{11}$ News about the inverse association between vegetables and fruit intake and HNC risk appeared online and in the newspaper Het Parool. ${ }^{12-14}$ Finally, our findings regarding vitamin C and HNC risk were broadcasted by TV Maastricht, a local television channel, in August 2015, and appeared on the known Dutch news website NU.nl. ${ }^{15,16}$ Hopefully, the announcement of our results at several conferences and in the media has contributed to creating awareness regarding lifestyle factors and HNC among both clinicians and the public.

Treatment decisions regarding HNC are complex, often with side effects resulting in significant morbidity with respect to basic functions such as speaking, swallowing, and breathing, as we wrote in Chapter 1 . For these reasons, we believe our results might contribute to individual health care. We hope our findings will eventually lead to a decrease in morbidity and mortality due to HNC that could possibly have been prevented. Through (future) health guidelines and increased awareness, we ultimately aim for the preservation of health and well-being of individual persons. The findings described in this thesis may also impact the community as a whole, by affecting the economic burden of health care provided to HNC patients as well as productivity loss at work due to health problems. Lastly, since the beneficial effects of a healthy lifestyle go beyond those respecting HNC risk and also influence other common (chronic) diseases such as cancer, CVD, and diabetes, the implications of a healthy lifestyle for health, well-being and healthcare may be even more far-reaching. ${ }^{2}$

As mentioned before, increased awareness about the relationship between lifestyle factors and health among both the public and clinicians is important. Ideally, information-for example by dietary guidelines-on how to eat and live healthy should be something to talk about in healthcare, at the general practitioner as well as in hospitals. Lately, there has been an increased interest in the relationship between lifestyle, nutrition, health, and disease prevention among public and clinicians, and there have been promising initiatives. Our own academic hospital, the Maastricht University Medical Center+, is a good example of this: its focus has been aimed at 'Healthy Living', including prevention, for a while now. ${ }^{17}$ Furthermore, together with the foundation Voeding Leeft ${ }^{18}$, two Dutch general practitioners established the 
Vereniging Arts en Voeding ${ }^{19}$ to increase national awareness about diet and health among clinicians, and encourage them to use this knowledge in their work. In addition, they plead for more health education and promotion, starting with more attention for nutrition, in medical studies. Another recent initiative is the release of the website Sick of Smoking $^{20}$, which is supported by patients, clinicians, midwifes and others, and aims to increase public debate regarding smoking. Earlier, two Dutch pulmonologists started the Stichting Rookpreventie Jeugd ${ }^{21}$, helped set up the website TabakNee ${ }^{22}$, and held a TEDx talk ${ }^{23}$ in their mission to stop people from smoking in 2013.

In view of the foregoing, however, it has to be noted that it is not easy to put health recommendations into practice for everyone. Especially since we live in an environment that constantly offers challenges in making healthy lifestyle choices, with unhealthy foods and products easily available, changing dietary or smoking habits remains difficult. Ideally, healthy foods and products should be plenty available in public institutions such as schools or railway stations. Thus, as described earlier regarding the WCRF NOURISHING framework ${ }^{5}$, food environment, food system, and behavioral change are important subjects for policy action.

\section{Precaution and future research}

For some lifestyle factors, the evidence is firmer than others. As one of the largest cohort studies on lifestyle factors and HNC risk so far, our findings regarding alcohol consumption, cigarette smoking, and intake of vegetables and fruits and HNC risk confirm and strengthen the existing evidence. In addition, these findings fit currentand probably add to future-dietary recommendations.

For other factors, however, we believe we should be more conservative before jumping to conclusions with regard to lifestyle recommendations or any clinical advice. We believe more research is warranted regarding the association between selenium and HNC risk, as described in Chapter 5. We also recommend future research to investigate the underlying mechanisms and to confirm our results regarding the association between intake of vitamins and carotenoids and HNC risk. Finally, future studies are warranted for further clarifications of the possible mechanisms regarding $\mathrm{BMI}$ and $\mathrm{HNC}$ risk. Other recommendations for further research have been described in Chapter 7. 


\section{Conclusion}

This chapter shows how the scientific findings described throughout this thesis might contribute to public health, healthcare, and future research and education. Our results could be used by policymakers, clinicians, researchers, and-last but not least-the public, to increase health and well-being and-ultimately-attain a decrease in the burden of disease and mortality due to preventable causes. In that view, our results may eventually lead to lower societal costs by reducing the health care burden related to HNC, as well as costs associated with productivity losses. Furthermore, our results provide important new leads for further research regarding lifestyle factors and HNC that could affect (future) health care and health guidelines as well. In conclusion, our results underline that prevention is a promising strategy in $\mathrm{HNC}$ and can be translated into societal value in many ways.

\section{References}

1 World Cancer Research Fund \& American Institute for Cancer Research. Food, Nutrition, Physical Activity and the Prevention of Cancer: a Global Perspective. American Institute for Cancer Research, 2007.

2 World Cancer Research Fund. Our Cancer Prevention Recommendations. London, UK: World Cancer Research Fund International. Available from: http://www.wcrf.org/int/research-we-fund/our-cancer-prevention-recommendations, accessed 15/07/2015.

3 World Cancer Research Fund. Continuous Update Project. London, UK: World Cancer Research Fund International. Available from: http://www.wcrf.org/int/research-we-fund/continuous-update-project-cup, accessed 15/07/2016.

4 American Institute for Cancer Research. 10 Recommendations for Cancer Prevention. Washington, USA: American Institute for Cancer Research. Available from: http://www.aicr.org/learn-more-about-cancer/infographics/10recommendations-for-cancer-prevention.html, accessed 15/07/2016.

$5 \quad$ World Cancer Research Fund. NOURISHING framework. London, UK: World Cancer Research Fund International. Available from: http://www.wcrf.org/int/policy/nourishing-framework, accessed 15/07/2016.

6 World Health Organization. Healthy diet. Geneva, Switzerland: World Health Organization. Available from: http://www.who.int/mediacentre/factsheets/fs394/en/, accessed 15/07/2016.

7 The Health Council of the Netherlands. Independent scientific advisory body for government and parliament. The Hague, The Netherlands: The Health Council of the Netherlands. Available from: https://www.gezondheidsraad.nl/en, accessed 15/07/2016

8 The Health Council of the Netherlands. Dutch dietary guidelines 2015. The Hague, The Netherlands: The Health Council of the Netherlands. Available from: https://www.gezondheidsraad.nl/en/publications/gezondevoeding/dutch-dietary-guidelines-2015, accessed 15/07/2016.

9 Netherlands Nutrition Centre. The Hague, The Netherlands: Netherlands Nutrition Centre. Available from: http://www.voedingscentrum.nl/nl.aspx, accessed 15/07/2016.

10 Netherlands Nutrition Centre. De Schijf van Vijf. The Hague, The Netherlands: Netherlands Nutrition Centre. Available from: http://www.voedingscentrum.nl/professionals/schijf-van-vijf/naslag-richtlijnen-schijf-van-vijf.aspx, accessed $15 / 07 / 2016$.

11 Metronieuws. Rol van alcohol bij mondkanker tot dusverre onderschat. Metronieuws. Available from: http://www.metronieuws.nl/nieuws/2014/05/rol-van-alcohol-bij-mondkanker-tot-dusverre-onderschat, accessed $15 / 07 / 2016$ 
12 Oncologisch onderzoek. Consumptie van groenten en fruit en risico van hoofd/halskanker in de Nederlandse Cohortstudie. Oncologisch onderzoek.nl. Available from: http://oncologischonderzoek.nl/page.php?al=Consumptievan-groenten-en-fruit-en+risico-hoofd\%2Fhalskanker-in-de-Nederlandse-Cohortstudie, accessed 15/07/2016.

13 Wereld Kanker Onderzoek Fonds. Meer groente en fruit, minder kans op kanker. Amsterdam, The Netherlands: Wereld Kanker Onderzoek Fonds. Available from: http://wkof.nl/nl/over-ons/voor-de-pers/persberichten/meergroente-en-fruit-minder-kans-op-kanker, accessed 15/07/2016.

14 Het Parool. Veel groente en fruit eten verkleint kans op mondholtekanker. Amsterdam, The Netherlands: Het Parool. Available from: http://www.parool.nl/amsterdam/veel-groente-en-fruit-eten-verkleint-kans-opmondholtekanker a4114075/, accessed 15/07/2016.

15 TV Maastricht. Vitamine $\mathrm{C}$ beschermt tegen mondholtekanker. Maastricht, The Netherlands: TV Maastricht (via Maastricht UMC+). Available from: http://www.mumc.nl/actueel/mumc-tv/specialismen/oncologie/4410834703001vitamine-c-beschermt-tegen-mondholtekanker, accessed 15/07/2016.

16 NU.nl. 'Vitamine C verlaagt mogelijk risco op mondholtekanker'. NU.nl. Available from: http://www.nu.nl/gezondheid/4101095/vitamine-c-verlaagt-mogelijk-risco-mondholtekanker.html, accessed 15/07/2016.

17 Maastricht UMC+. Healthy Living. Maastricht, The Netherlands: Maastricht UMC+. Available from: http://www.mumc.nl/over-mumc/het-maastricht-umc/kennis-limburg-verbindt/de-crossovers/healthy-living, accessed 15/07/2016

18 Voeding Leeft. Een nieuwe kijk op voeding. Amsterdam, The Netherlands: Voeding Leeft. Available from: http://voedingleeft.nl/, accessed 15/07/2016.

19 Vereniging Arts en Voeding. Available from: http://www.artsenvoeding.nl/, accessed 15/07/2016.

20 Sick of Smoking. Stop de tabaksindustrie. Available from: http://sickofsmoking.nl/, accessed 15/07/2016.

21 de Kanter W. \& Dekker P. Stichting Rookpreventie Jeugd. Amsterdam, The Netherlands: Stichting Rookpreventie Jeugd. Available from: http://stichtingrookpreventiejeugd.nl/, accessed 15/07/2016.

22 Stichting Rookpreventie Jeugd. TabakNee. Amsterdam, The Netherlands: Stichting Rookpreventie Jeugd. Available from: http://www.tabaknee.nl/, accessed 15/07/2016.

23 de Kanter W. \& Dekker P. Replacement smokers. Nijmegen, The Netherlands: TEDx. Available from: http://tedxnijmegen.nl/2013/04/wanda-de-kanter-en-pauline-dekker-the-industry-should-be-ashamed/, accessed $15 / 07 / 2016$. 
Dankwoord 
Promoveren doe je niet alleen! Allereerst ben ik mijn promotieteam dankbaar dat ik deel heb mogen uitmaken van dit project en me zo de afgelopen jaren verder heb kunnen ontwikkelen, zowel op professioneel als persoonlijk vlak. Ook wil ik alle NLCS deelnemers hartelijk danken. Zonder hen was dit onderzoek niet mogelijk geweest!

Piet, als mijn $1^{\mathrm{e}}$ promotor wil ik je van harte bedanken voor je begeleiding gedurende mijn promotietraject. Je kennis, deskundigheid, scherpe blik, en concrete opmerkingen en adviezen heb ik erg gewaardeerd. Ook de tijdslijn hield je goed in de gaten; als ik de planning te optimistisch inschatte, moest je me soms afremmen ;-). Bedankt!

Bernd, mijn $2^{\mathrm{e}}$ promotor, ook jou wil ik ontzettend bedanken! Jouw klinische kijk op ons project vond ik altijd erg fijn. Daarnaast heb je me enorm geïnspireerd met je menselijke blik, vriendelijkheid en hartelijkheid, niet alleen in je doen en laten, maar ook als je vertelde over je patiënten en je gezin. Onze reis naar Griekenland en het congres daar waren zeer geslaagd en hebben mooie herinneringen opgeleverd $(;)$ ! Leo, mijn copromotor, bedankt dat ik altijd voor overleg bij je terecht kon! Je was niet alleen inhoudelijk in de data, analyses en teksten geïnteresseerd, maar ook in hoe het met deze AIO persoonlijk ging. Deze betrokkenheid heb ik erg gewaardeerd. Inmiddels ben jij er als begeleider achter dat het AIO-schap een tijd is die op meerdere fronten zijn vruchten kan afwerpen ;-), maar dat een promotietraject-ondanks een bijgestelde planning-uiteindelijk meestal toch op zijn pootjes terechtkomt!

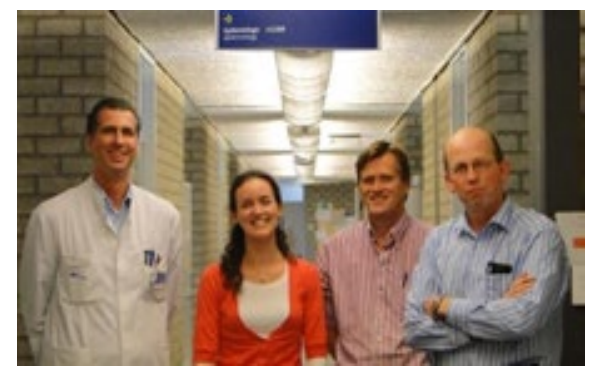

Promotieteam: bedankt!

Co-auteurs: Sandra, dank voor je waardevolle input voor het project! Jouw scherpe blik is de artikelen zeker ten goede gekomen. Leonie, bedankt voor je bijdrage aan het tot stand komen van het vitaminen en carotenoïden artikel! Je hebt als stagiaire een hele mooie prestatie neergezet, en ik ben blij dat ik je hierin mocht begeleiden.

Leden van de beoordelingscommissie, prof. dr. Speel, dr. van Dijk, prof. dr. Kessler, prof. dr. Kremers en prof. dr. van der Laan: hartelijk dank voor de aandacht die jullie besteed hebben aan het kritisch lezen en beoordelen van dit proefschrift. 
Collega's Epidemiologie: ik heb de afgelopen jaren met plezier bij jullie gewerkt! In het bijzonder wil ik Yvonne, Mariëlle en Petra bedanken voor hun hulp op secretarieel vlak, alsook voor de gezellige gesprekken en het mogen lenen van zwangerschapskleding ;-). Harry en Jos, zonder jullie ICT hulp had ik het op een aantal momenten in mijn promotietraject waarschijnlijk niet gered! Het NLCS team: bedankt voor al jullie inspanningen! Jolanda, jou wil ik daarnaast bedanken voor je hulp bij het plannen van afspraken, wat niet altijd makkelijk was ;-). Je humor en positieve inslag waardeer ik enorm! Ook andere collega's met wie ik heb samengewerkt of regelmatig gezellige praatjes in het keukentje/de postkamer/het toilet over privé-perikelen en alle ins en outs van de afdeling heb gemaakt: BEDANKT!

'Jong EPID': mede dankzij jullie kijk ik met heel veel plezier terug op mijn AIO-tijd! Ik vind het bijzonder dat we zo'n fijne club (oud-)promovendi waren en zijn. Thembilunches, de PhD tour, jullie support bij mijn Lingo-avontuur, de vele etentjes, drankjes, feestjes en zelfs oudjaarsavonden die we gedeeld hebben: ik kijk er met plezier op terug, en kijk uit naar alles wat nog komen gaat! In het bijzonder wil ik mijn mede 'LAIO's' (Leo's AIOS!) Anne en Ivette, mijn voormalige bovenbuurvrouw Nadine (beter een goede buur dan een verre vriend, zeker als die buur geweldig kan koken!), mijn huidige buurman Huub

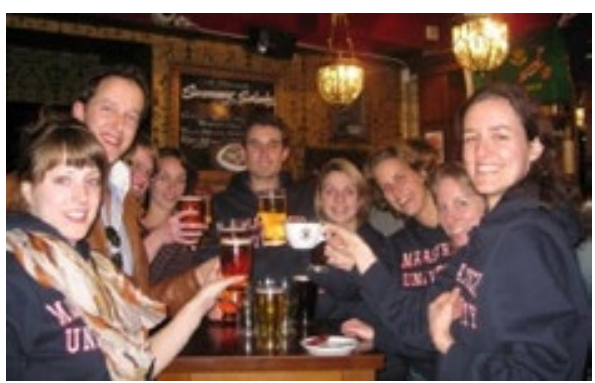

Epi's on Tour! (redder in nood bij het plaatsen van tabellen!), mijn 'roomies' van het eerste uur, Vivian en Emmylou, en Colinda en Rachel (onder meer voor een fantastisch 'fashion' weekend Amsterdam!) bedanken. Op naar de 'oud EPID' club ;-)!

Mijn paranimfen, Eline en Karolina: Eline, bedankt voor alle jaren gezelligheid in ons 'Tropical Paradise' op Deb 1! Ik kon niet alleen voor persoonlijke, maar ook inhoudelijke (lees: statistiek (:) ) dingen bij je terecht. Dank je wel voor alles wat we samen gedeeld hebben! Karolina, dat laatste geldt ook voor jou: bedankt voor alle fijne gesprekken, wandelingen, drankjes, dansjes en meer! Ik ben blij dat ik jullie de afgelopen jaren heb leren kennen en dat jullie straks-letterlijk;-)-achter me staan!

Familie en vrienden: bedankt voor jullie belangstelling in mijn promotieonderzoek! Jaarclub (Zuid), GAIA, 'Date-chicks', 'Donne Italiane' en andere vrienden en familie: dank voor alle gezelligheid (taartjes, koffietjes, etentjes etc.!) en fijne middagen, 
avonden en weekendjes! Ongetwijfeld hebben die bijgedragen aan het afronden van dit boekje (:). Esther ('Illy'!), vanaf het allereerste begin in Maastricht-de facultaire introductie bij GW-klikte het tussen ons, en dat is altijd zo gebleven! Sindsdien hebben we samen van alles meegemaakt: onze studies gezondheidswetenschappen en geneeskunde, KoKo, de vele gezellige jaarclubavonden, vakanties, co-schappen in Eindhoven, en nog veel meer lief en leed ;-). Fijn dat je er altijd voor me bent!

Een speciaal woord van dank ook aan opa en oma van Zwam en oma Maasland. Opa, ik ben blij dat ik je met je 91 jaar nog steeds om me heen mag hebben. Jouw levenslust is een grote inspiratie voor me! Oma Maasland, met uw 97 jaar bent $u$ altijd een voorbeeld voor me geweest om tevreden en dankbaar in het leven te staan. Op dit moment gaat het helaas niet goed met $u$, en waarschijnlijk zult $u$ de promotie niet meer meemaken. Dank u wel voor alle mooie jaren!

Naast vrienden en familie zijn er ook anderen-waaronder mijn THHM-collega's-die de afgelopen jaren (indirect) hebben bijgedragen aan mijn proefschrift. Bedankt!

Mijn ouders: jullie hebben me altijd gestimuleerd om te studeren, wat uiteindelijk tot dit boekje geleid heeft. Daarnaast staan jullie altijd voor mij en ons klaar, niet alleen als ouders, maar inmiddels ook als trotse opa en oma (-). Dat heeft-zeker de laatste weken!-veel bijgedragen aan dit boekje. Bedankt voor alles!

Rik en Daan, ik ben blij met jullie als broers! Als wijze grote zus;-) ben ik trots op wat jullie bereikt hebben. Fijn dat we altijd zo kunnen lachen om hele slechte grappen! Als jullie je afvragen waar ik nu al die jaren mee bezig ben geweest: zie hier het antwoord! Sushma, het is altijd gezellig als je er bent! Ik ben blij met jou als (aanstaande) schoonzus en voel me vereerd dat ik binnenkort als getuige naast Rik en jou mag staan. Mijn schoonfamilie: ook jullie wil ik hartelijk bedanken voor jullie interesse in en het meeleven met mijn promotieonderzoek. Dank jullie wel!

Lieve Servaas, bedankt voor je onvoorwaardelijke steun en liefde, ook in dit promotietraject. In het bijzonder bedankt voor alle worteljuices en koffietjes die me door het afronden van de thesis heen geholpen hebben ;-)! Dank je wel dat je er altijd voor me bent! Lieve Iza, ik ben ontzettend blij en dankbaar jouw mama te mogen zijn. Jouw lieve lach en vrolijkheid (en inmiddels ook 'boevigheid'!) maken elke dag bijzonder. Ik hou ontzettend veel van jullie en kijk uit naar ons verdere leven samen $(;)$ !

\section{Denise}

Maastricht, augustus 2016 
Curriculum Vitae 


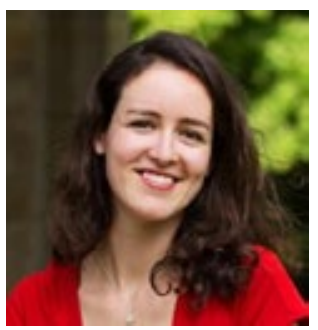

Denise Maasland was born on August 11, 1983, in Heerlen, the Netherlands. After completing secondary school (Gymnasium; Strabrecht College, Geldrop) in 2001, she studied Health Sciences (2001-2006) and Medicine (20022009) at Maastricht University.

Regarding Health Sciences, Denise wrote her bachelor's thesis 'The role of growth factors in the development of breast cancer: possibilities for prevention' in 2004. She performed her master's internship 'The Role of Angiogenesis in B-cell lymphoma' at the Department of Pathology (Maastricht University Medical Center+). In May 2006, Denise obtained her master's degree in Health Sciences, with a specialization in Biological Health Sciences.

During medical school, Denise did her elective in pediatric oncology (The Royal Children's Hospital/The Children's Cancer Center, Melbourne, Australia) and performed her senior internship in radiation oncology (Catharina Hospital, Eindhoven). After obtaining her medical degree in August 2009, Denise worked as a doctor in youth public health (Jeugdgezondheidszorg; Public Health Service (GGD), Eindhoven) and as a resident-not-in-training (ANIOS) in pediatrics and psychiatry (Zuyderland, Heerlen).

In 2011, Denise started her PhD project 'Lifestyle factors and risk of head-neck cancer subtypes: a prospective cohort study' at Maastricht University, supervised by prof. dr. ir. Piet van den Brandt, prof. dr. Bernd Kremer, and dr. Leo Schouten. She performed her research at the Department of Epidemiology, in collaboration with the Department of Otorhinolaryngology, Head \& Neck Surgery (Maastricht University Medical Center+). Since 2012, Denise has also been volunteering as supervisor of a psychosocial support group for young adults with cancer at the Toon Hermans Huis Maastricht.

With her ongoing interest in mental and physical health and their relationship with lifestyle, Denise aims to pursue a career in psychotherapy and health counseling. 
List of publications 


\section{Published}

1 Maasland DHE, Schouten LJ, Kremer B, van den Brandt PA. Toenail selenium status and risk of subtypes of head-neck cancer: the Netherlands Cohort Study. Eur J Canc 2016;60:83-92.

2 Maasland DHE, van den Brandt PA, Kremer B, Schouten $L$. Body mass index and risk of subtypes of head-neck cancer: the Netherlands Cohort Study. Sci Rep 2015;5:17744.

3 De Munter L, Maasland DHE, van den Brandt PA, Kremer B, Schouten L. Vitamin and carotenoid intake and the risk of head-neck cancer subtypes in the Netherlands Cohort Study. Am J Clin Nutr 2015;102:420-32.

4 Maasland DHE, van den Brandt PA, Kremer B, Goldbohm RA, Schouten L. Consumption of vegetables and fruits and risk of subtypes of head-neck cancer in the Netherlands Cohort Study. Int J Cancer 2015;136:E396-409.

5 Maasland DHE, van den Brandt PA, Kremer B, Goldbohm RA, Schouten L. Alcohol consumption, cigarette smoking and the risk of subtypes of head-neck cancer: results from the Netherlands Cohort Study. BMC Cancer 2014;14:187.

\section{Submitted}

6 Perloy A, Maasland DHE, van den Brandt PA, Kremer B, Schouten L. Intake of meat and fish and risk of head-neck cancer subtypes in the Netherlands Cohort Study.

\section{Oral presentations}

7 Body mass index and risk of subtypes of head-neck cancer: results from the Netherlands Cohort Study. 227th General Meeting of the Dutch Otorhinolaryngology Society. Nieuwegein, The Netherlands; November 2015.

8 Consumption of vegetables and fruits and risk of subtypes of head-neck cancer: results from the Netherlands Cohort Study. 223rd General Meeting of the Dutch Otorhinolaryngology Society. Maastricht, The Netherlands; November 2013.

9 Alcohol consumption, cigarette smoking and risk of subtypes of head-neck cancer: results from the Netherlands Cohort Study. 4th World Congress of the International Academy of Oral Oncology. Island of Rhodes, Greece; May 2013.

10 Alcohol consumption, cigarette smoking and risk of subtypes of head-neck cancer: results from the Netherlands Cohort Study. 221st General Meeting of the Dutch Otorhinolaryngology Society. Nieuwegein, The Netherlands; November 2012.

11 Lifestyle factors and risk of head-neck cancer. PhD tour of Epidemiology (interactive tour of PhD students from Maastricht University, Department of Epidemiology, to the Departments of Epidemiology of University College London, the University of Oxford, and the University of Birmingham). Birmingham, United Kingdom; October 2011.

12 Lifestyle factors and risk of head-neck cancer. Rank Prize Funds: Mini-symposium on Alcohol and Nutrition. Grasmere, United Kingdom; July 2011.

\section{Poster presentation}

13 Alcohol consumption, cigarette smoking and risk of subtypes of head-neck cancer: results from the Netherlands Cohort Study. WEON (annual Dutch Epidemiology Conference). Rotterdam, The Netherlands; June 2012. 

